\title{
AN INVESTIGATION OF THE LEARNING AND \\ FORGETTING OF WORKERS IN DUAL RESOURCE \\ CONSTRAINED SYSTEMS
}

by

John Russell Zamiska

B. Eng., Industrial Engineering, June 2001

Ryerson University, Toronto, Ontario

A thesis presented to

Ryerson University

in partial fulfillment of the

requirements for the degree of

Master of Applied Science

in

Mechanical Engineering

Toronto, Ontario, Canada, 2004

(C) John Zamiska 2004 


\section{UMI Number: EC52994}

\section{All rights reserved \\ INFORMATION TO USERS}

The quality of this reproduction is dependent upon the quality of the copy submitted. Broken or indistinct print, colored or poor quality illustrations and photographs, print bleed-through, substandard margins, and improper alignment can adversely affect reproduction.

In the unlikely event that the author did not send a complete manuscript and there are missing pages, these will be noted. Also, if unauthorized copyright material had to be removed, a note will indicate the deletion.

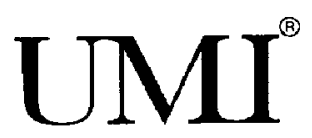

UMI Microform EC52994

Copyright 2008 by ProQuest LLC

All rights reserved. This microform edition is protected against unauthorized copying under Title 17, United States Code.

ProQuest LLC

789 East Eisenhower Parkway

P.O. Box 1346

Ann Arbor, Ml 48106-1346 

Ryerson University requires the signatures of all persons using or photocopying this thesis. Please sign below, and give address and date. 


\title{
AN INVESTIGATION OF THE LEARNING AND FORGETTING OF WORKERS IN DUAL RESOURCE CONSTRAINED SYSTEMS
}

\begin{abstract}
A thesis presented to Ryerson University in partial fulfillment of the requirements for the degree of Master of Applied Science in Mechanical Engineering, John Zamiska, 2004
\end{abstract}

\section{ABSTRACT}

This thesis examines worker learning and forgetting in dual resource constrained systems according to the dual-phase learning-forgetting model (DPLFM). The contributions are as follows: (1) equations were developed that output controllable shop factors such as training and transfer policies given existing factors such as the degree of job similarity, processing times, and the learning and forgetting rate of the worker, (2) results suggest that the task-type factor with respect to the worker learning rate and proportion of cognitive and motor elements is a factor to include in DRC research, and (3) the results have suggested that the DPLFM emphasized a greater benefit for upfront training and more a frequent transfer policy than the learn forget curve model (LFCM) when tasks are similar, and supported the conclusions of Jaber et al. (2003) by an even greater extent that it is possible to use more flexibility in DRC shops with similar tasks. 


\section{ACKNOWLEDGEMENTS}

I would like to gratefully acknowledge the support, encouragement, knowledgeable advice, and patience generously granted to me by my supervisor professor Mohamad Y. Jaber. I would also like to thank professor. P. Gharghouri, Dr. L. Kolasa, and Dr. B. Todorow for their creative advice. Also, I would like to thank the Natural Sciences and Engineering Research Council of Canada (NSERC) for supporting me financially through this initiative.

Finally, I would like to thank all my family and friends that have supported me through this endeavour. 


\section{TABLE OF CONTENTS}

Author's declaration .............................................................ii

Borrower's page $\quad$.............................................................iii

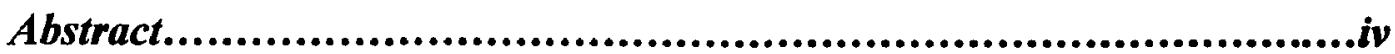

Acknowledgements.........................................................v

Table of Contents.............................................................vi

List of Tables............................................................x

List of Figures...........................................................xi

Nomenclature...............................................................xiii

Chapter 1: introduction and literature review........................................

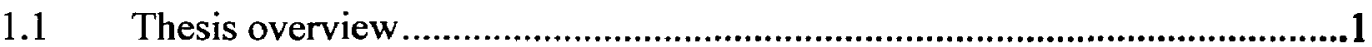

1.2 Dual resource constrained (DRC) systems ........................................................

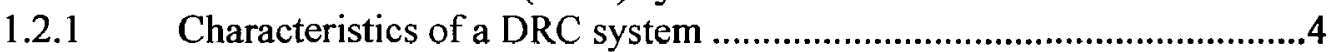

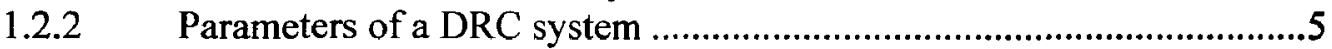

1.2.3 DRC research: an overview ...............................................................

1.3 Learning and forgetting models..................................................................12

1.3.1 The learning phenomenon - an introduction .......................................12

1.3.2 Learning and forgetting research: an overview...................................20

$1.4 \quad$ Learning and forgetting in DRC systems: an overview...............................24

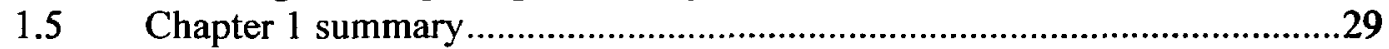

Chapter 2: the dual-phase learning-forgetting model (DPLFM) ....................................30

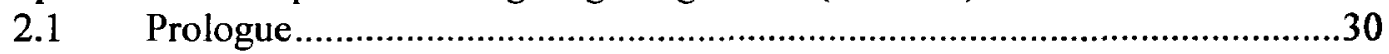

2.1.1 The dual-phase learning model (DPLM) …………….........................31

2.1.2 The learn-forget curve model (LFCM) .................................................33

$2.2 \quad$ The dual-phase learning-forgetting model (DPLFM) ...................................35

2.2.1 Numerical example of the DPLFM........................................................

$2.3 \quad$ Chapter 2 summary ............................................................................4

Chapter 3: experimental design and modeling..........................................................42

3.1 Experimental design of model A ……………............................................42

3.1.1 Recognition and statement of the problem .........................................43

3.1.2 Choice of factors, levels, and ranges.....................................................43

3.1.3 Selection of the response variables .....................................................49

3.1.4 Choice of experimental design..............................................................49

3.1.5 Modeling methodology ..........................................................................49

3.2 Experimental design of model B .................................................................50

3.2.1 Recognition and statement of the problem .........................................51

3.2.2 Choice of factors, levels, and ranges.................................................52

3.2.3 Selection of the response variables .....................................................52

3.2.4 Choice of experimental design ..............................................................54

3.2.5 Modeling methodology ...........................................................................54

3.3 Experimental design of model C ................................................................54 
3.3.1 Recognition and statement of the problem .......................................55

3.3.2 Choice of factors, levels, and ranges...................................................55

3.3.3 Selection of the response variables.................................................55

3.3.4 Choice of experimental design......................................................55

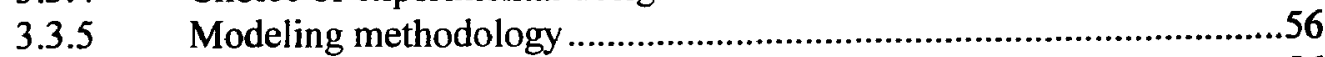

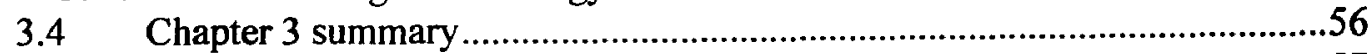

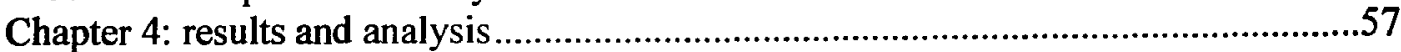

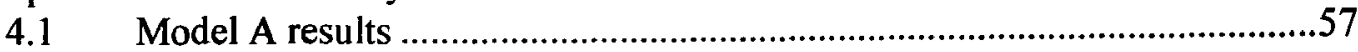

4.1.1 The main effect analysis of model A ...............................................58

4.1.1.1 Description of the main effect ....................................................58

4.1.1.2 Discussion of the main effect (the VRVF, the LFCM, and the

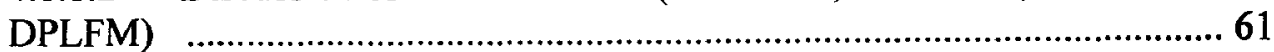

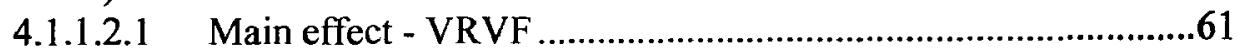

4.1.1.2.2 Main effect - LFCM ..........................................................61

4.1.1.2.3 Main effect - DPLFM .....................................................64

4.1.1.3 Main effect conclusions.........................................................66

4.1.2 The interaction effect analysis of model A .......................................67

4.1.2.1 Description of the interaction effect ............................................67

4.1.2.2 Discussion of the interaction effect (LFCM and DPLFM).............70

4.1.2.2.1 The interactions between $C$ and $F R$..................................70

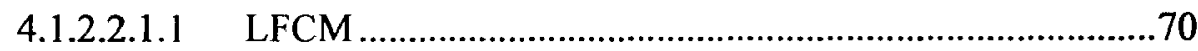

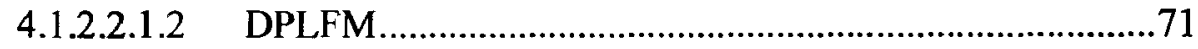

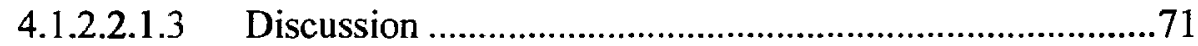

4.1.2.2.2 The interactions between $C$ and $F A P$..............................71

4.1.2.2.2.1 LFCM ................................................................... 71

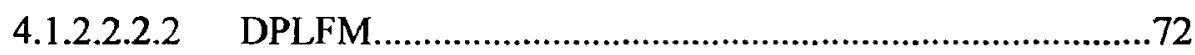

4.1.2.2.2.3 Discussion ............................................................... 72

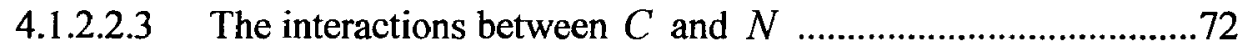

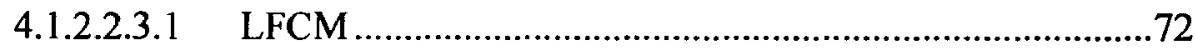

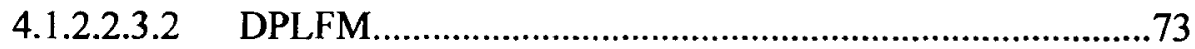

4.1.2.2.3.3 Discussion ................................................................73

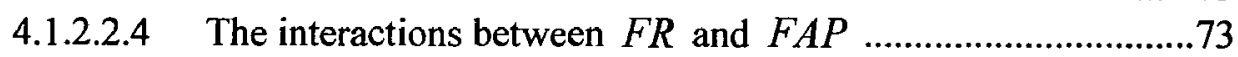

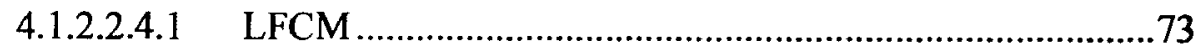

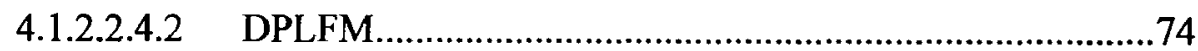

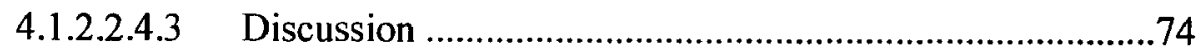

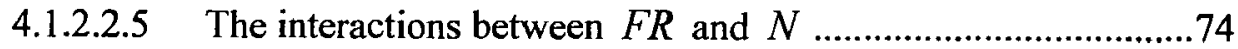

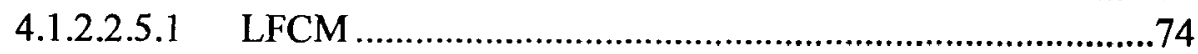

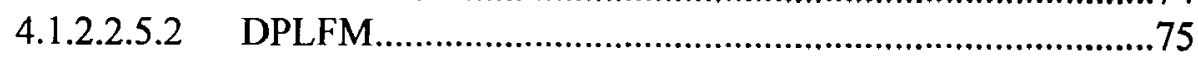

4.1.2.2.5.3 Discussion ...............................................................75

4.1.2.2.6 The interactions between $F A P$ and $N$............................75

4.1.2.2.6.1 LFCM .....................................................................75

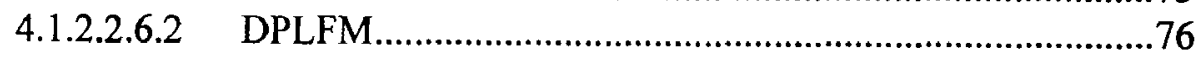

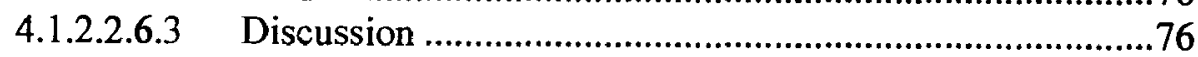

4.1.2.2.7 Summary of the interaction effect (LFCM and DPLFM) .............

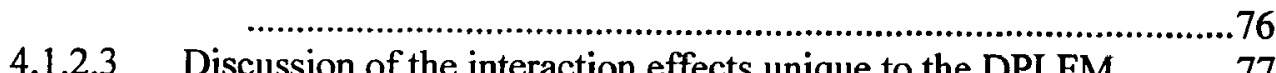

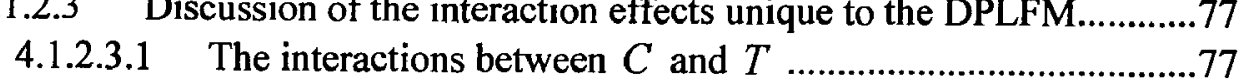

4.1.2.3.1.1 Discussion .................................................................78

4.1.2.3.2 The interactions between $C$ and $R$....................................78

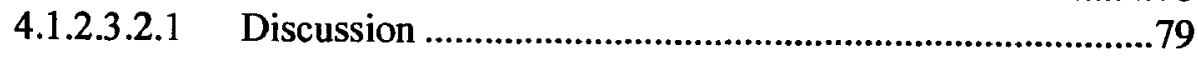


4.1.2.3.3 The interactions between $F R$ and $T$..................................79

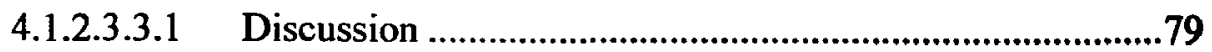

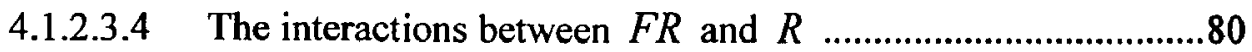

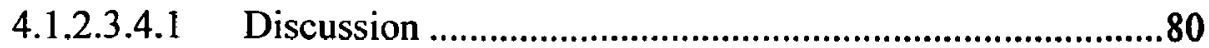

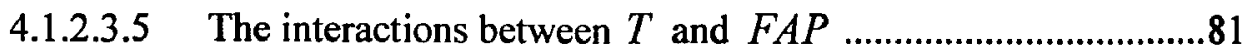

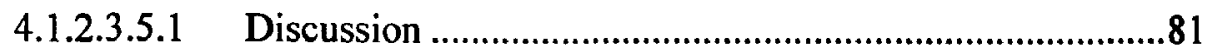

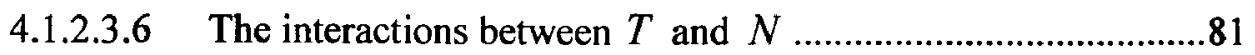

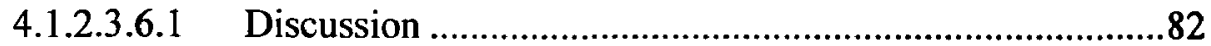

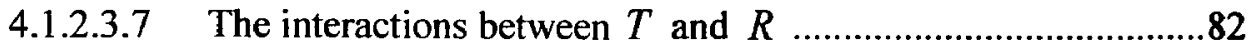

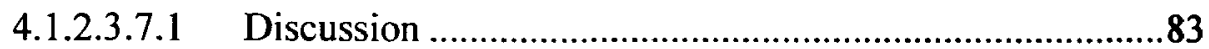

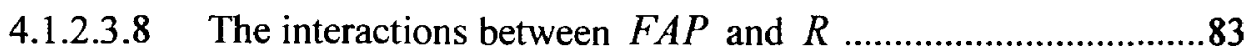

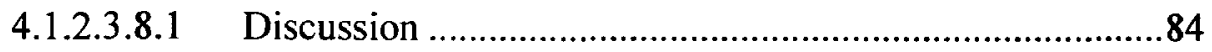

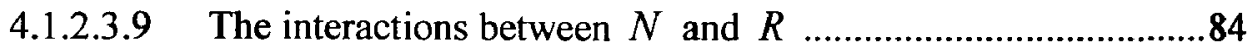

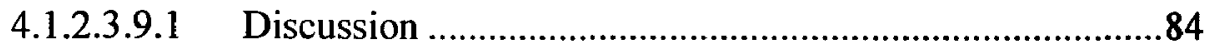

4.1.2.4 Interaction effect conclusions - DPLFM ......................................85

4.1.3 Comparison of the results of model A with Jaber et al. (2003) ...........86

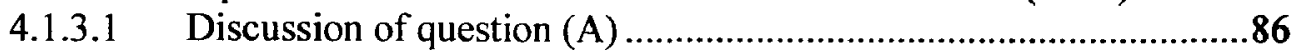

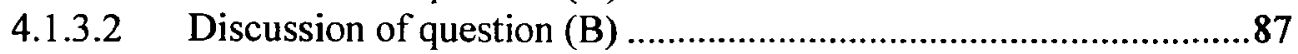

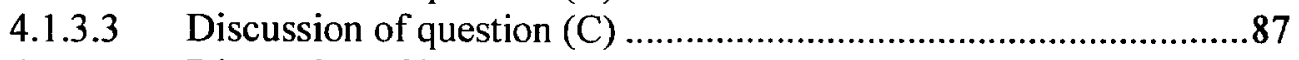

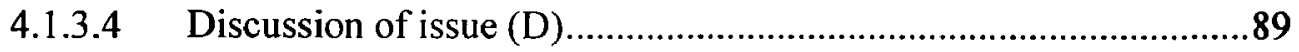

4.1.4 Implications of results for the five DRC issues ..................................90

4.1.4.1 Issue 1: worker flexibility (number of tasks learned) .......................90

4.1.4.2 Issue 2: centralization of control ('when' rule) ..............................90

4.1.4.3 Issue 3: worker assignment..........................................................91

4.1.4.4 Issue 4: queue disciplines ............................................................91

4.1.4.5 Issue 5: cost of transferring workers...............................................91

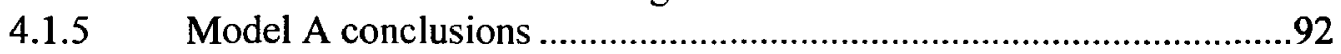

4.2 Model B results...............................................................................94

4.2.1 Comparison of the results of model B with Jaber et al. (2003)............94

4.2.2 Implication of results for the five DRC issues .....................................99

4.2.2.1 Issue 1: worker flexibility (number of tasks learned) ......................100

4.2.2.2 Issue 2: centralization of control ('when' rule) …...........................100

4.2.2.3 Issue 3: worker assignment..........................................................100

4.2.2.4 Issue 4: queue disciplines ............................................................. 100

4.2.2.5 Issue 5: cost of transferring workers................................................101

4.2.3 Model B conclusions..................................................................... 101

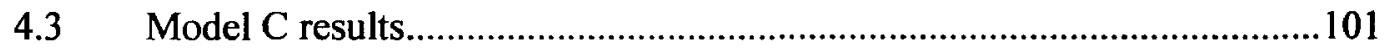

4.3.1 Preliminary main effect analysis of $C^{*}$ and $F A P^{*}$..........................101

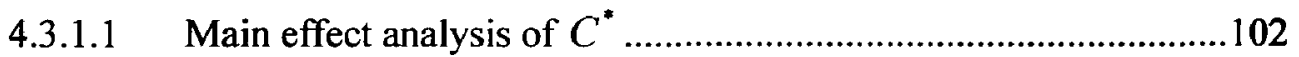

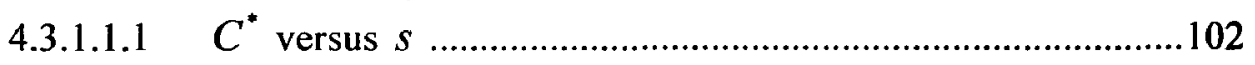

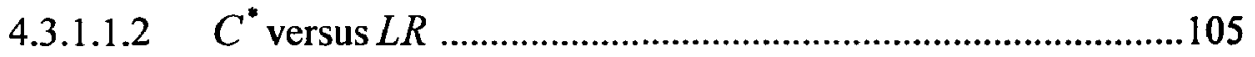

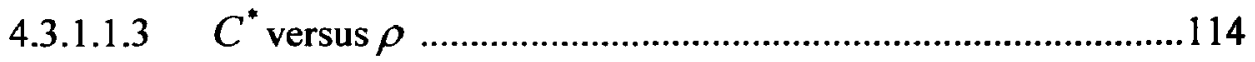

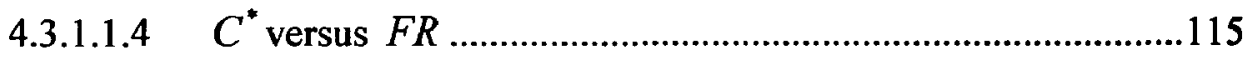

4.3.1.2 Main effect analysis of $F A P^{*}$..................................................117

4.3.1.2.1 $F A P^{*}$ versus $s$............................................................117

4.3.2 Logistic regression equations and numerical example ........................121

4.3.2.1 Prologue. 
4.3.2.2 Numerical example - the application of the logistic equations

4.3.2.2.1 The logistic equations

4.3.2.2.2 Description of the pseudo DRC shop problems ......................127

4.3.2.2.2.1 DRC shop problem 1 ..............................................127

4.3.2.2.2.2 DRC shop problem 2 ...............................................127

4.3.2.2.3 Solution of DRC shop problem 1 ..........................................128

4.3.2.2.4 Solution of DRC shop problem 2 ........................................130

4.3.3 Implications of results for the five DRC issues ..............................133

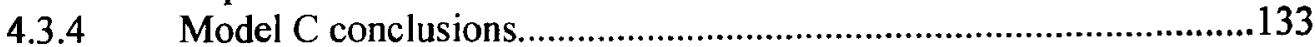

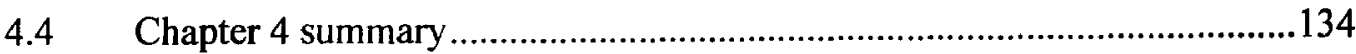

Chapter 5: thesis conclusions and recommendations for further research ..................135

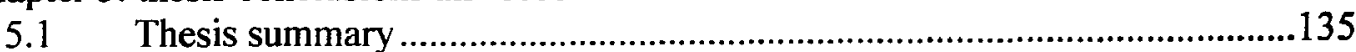

5.2 Thesis contributions to DRC research ..................................................136

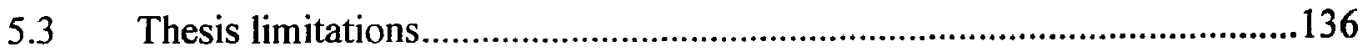

$5.4 \quad$ Recommendations for further research..............................................138

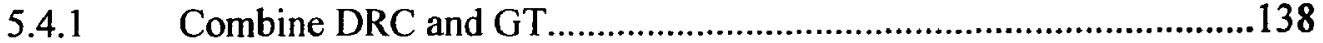

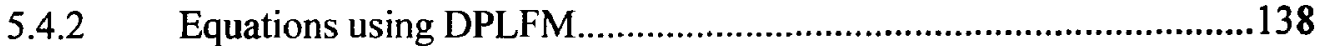

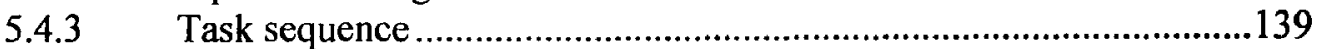

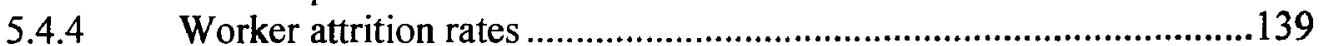

5.4.5 Further recommendations - DRC summary matrix...........................140

5.4.5.1 DRC summary matrix overview................................................ 140

5.4.5.2 Further recommendations for DRC research.............................141

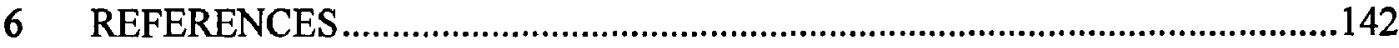

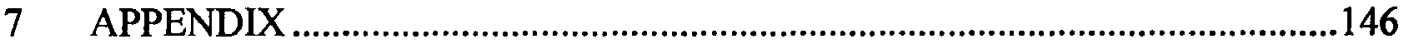

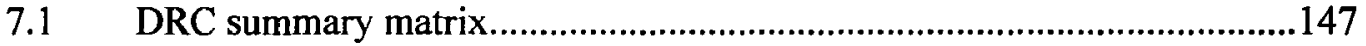

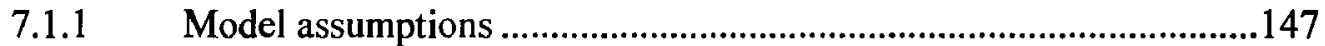

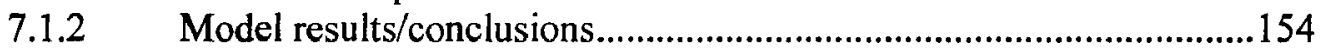

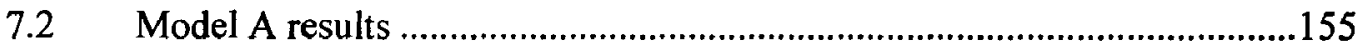

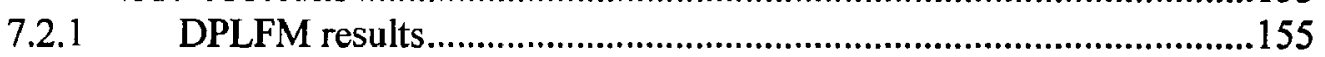

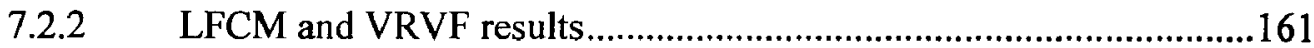

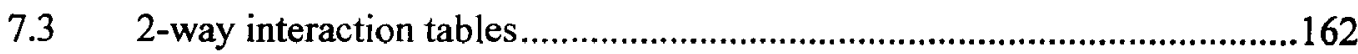

7.3.1 2-way DPLFM interaction tables.................................................162

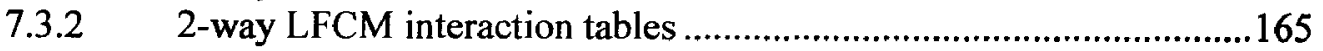

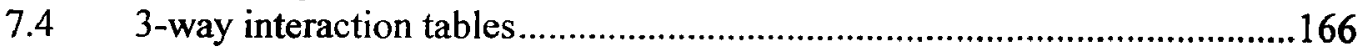

7.4.1 3-way DPLFM interaction tables.....................................................166

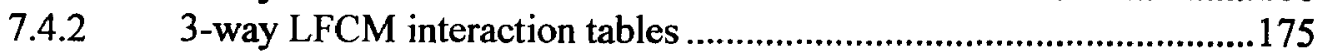

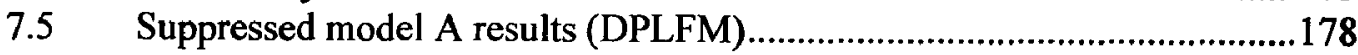

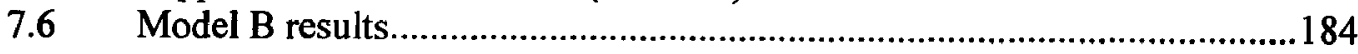

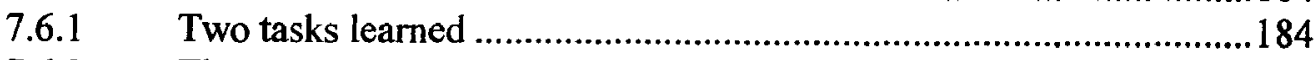

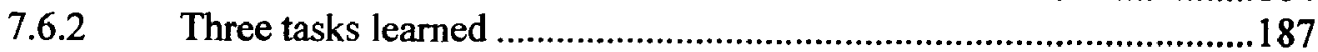

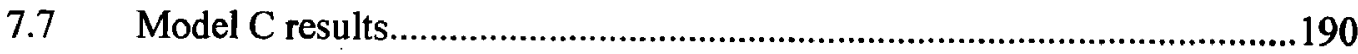

7.8 The Hosmer-Lemeshow goodness of fit tests for the binary logistic equations of model $\mathrm{C}$ 


\section{LIST OF TABLES}

Table 2.1: Parameter estimations for the DPLFM numerical example......................................36

Table 2.2: Initial five production cycle parameters of the DPLFM numerical example ..............41

Table 3.1: Experimental factors used in the study of Jaber et al. (2003) .....................................44

Table 3.2: Additional experimental factors used in model A .....................................................46

Table 3.3: Additional parameters related to time-for-total-forgetting used in model A..............48

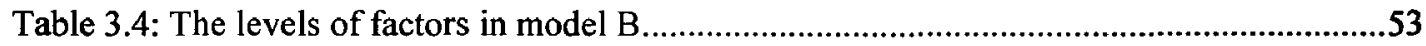

Table 3.5: Input and output factors used in the formulation of the multivariate function

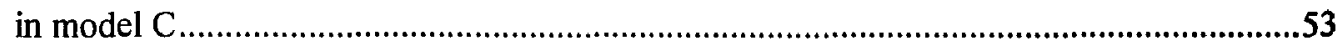

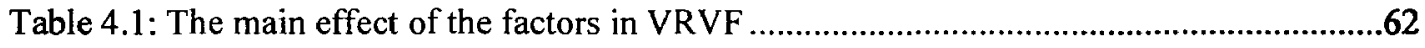

Table 4.2: The main effect of the factors in LFCM....................................................................63

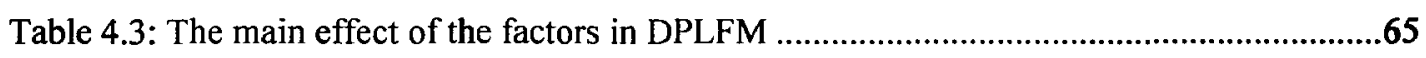

Table 4.4: Factors, their acronyms, and their interactions for model A A..................................68

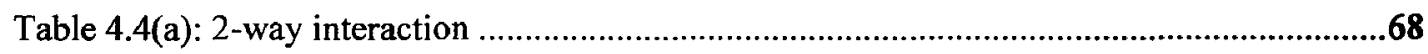

Table 4.4(b): 2-way interaction with relative difference from baseline ..................................68

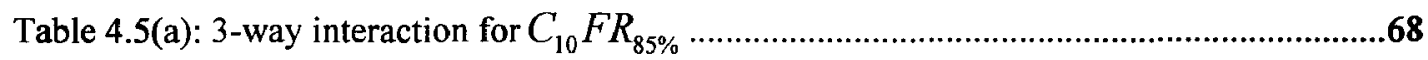

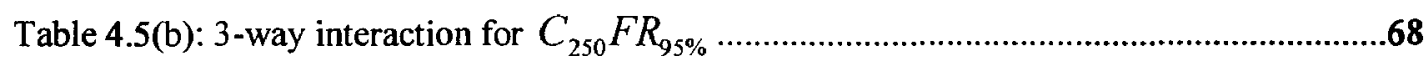

Table 4.6: Percent decrease in average processing times with increasing task similarity -

DPLFM versus $\operatorname{LFCM}(N=2)$..

Table 4.7: Suppressed average processing time results for the DPLFM with the task similarity factor $-C=10, N=3$.

Table 4.8: Percent decrease in average processing times with increasing task similarity -

DPLFM versus $\operatorname{LFCM}(N=3)$.

Table 4.9: Average processing times with respect to number of tasks learned and task similarity factor - DPLFM and LFCM

Table 4.10: Main effects of each input variable versus level of $C^{*}$

Table 4.11: Main effects of each input variable versus level of $F A P^{*}$

Table 4.12: Summary of results of numerical example for the binary logistic regression equation of model C 


\section{LIST OF FIGURES}

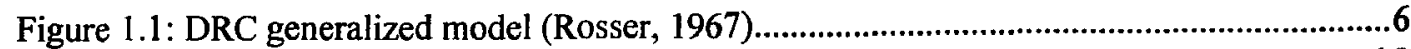

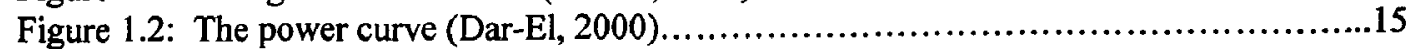

Figure 1.3: Log-log linear form of the power curve (Dar-El, 2000)...........................................15

Figure 1.4: The Stanford B model (Dar-El, 2000) ..................................................................17

Figure 1.5: The behaviour of common learning models (Jaber, 1996)........................................17

Figure 2.1: Prediction based on early data (Dar-El, 2000) ...........................................................32

Figure 2.2: Prediction based on late data (Dar-El, 2000) ...........................................................32

Figure 2.3: The combined effect on learning performance (Dar-El et al., 1995)..........................32

Figure 3.1: Classification of learning slopes (Dar-El et al, 1995) ...............................................46

Figure 3.2: The ranges of learning rates for the four task-types in model $A$.................................48

Figure 4.1: Graphs displaying the relative main effects between learning models.......................59

Figure 4.2; Changes in the relative main effect between learning-forgetting models ..................60

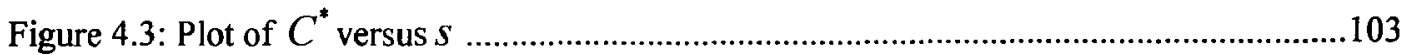

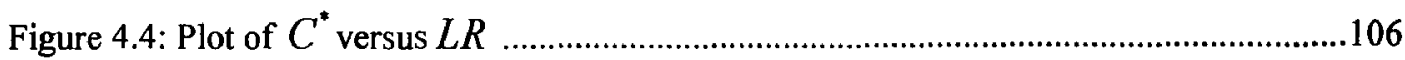

Figure 4.5: Average processing time ( $A P T$ ) versus learning rate $(F A P-0) \ldots \ldots \ldots \ldots \ldots \ldots . . . .106$

Figure 4.6: Interaction between type of driver and type of beverage for the golf experiment

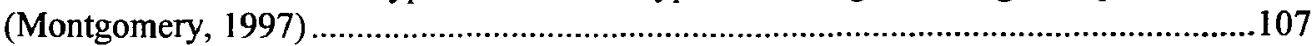

Figure 4.7: Average processing time ( $A P T$ ) versus learning rate $(F A P-1)$......................107

Figure 4.8: Average processing time ( $A P T$ ) versus learning rate for several values of $C$ averaged across $F A P-0$ and $F A P-1(N=0, N=2, \rho=4) \ldots \ldots \ldots \ldots \ldots \ldots \ldots \ldots . . . . . . .107$

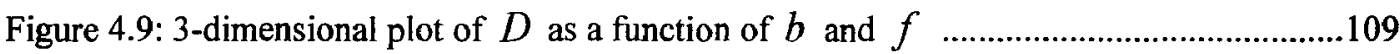

Figure 4.10: Plot of Average processing time ( $A P T$ ) versus $C$ for all $F A P-1$ values averaged across all forgetting rate levels at $\rho=4(s=0, N=2)$

Figure 4.11: Time to total forgetting $(D)$ in the first cycle versus learning rate for different levels of initial training ( $C=100, F R=88 \%, s=0, N=2, \rho=4)$

Figure 4.12: Time for total forgetting $(D)$ in the first cycle versus learning rate for different $\rho$

-values ( $C=100, F A P-1, F R=88 \%, s=0, N=2)$

Figure 4.13: Plot of $C^{*}$ versus $\rho$

Figure 4.14: 3-dimensional plot of average processing time ( $A P T$ ) as a function of $\rho$ and $C$ with ranges of $2<\rho<6$ and $100<C<300$; where $b$ and $f$ are kept constant at $0.152(L R=90 \%)$ and $0.322(F R=80 \%)$ respectively

Figure 4.15: 3-dimensional plot of average processing time ( $A P T$ ) as a function of $\rho$ and $C$ with ranges of $2<\rho<6$ and $100<C<300$; where $b$ and $f$ are kept constant at $0.515(L R=70 \%)$ and $0.074(F R=95 \%)$ respectively. 115

Figure 4.16: Plot of $C^{*}$ versus $F R$ .116

Figure 4.17:3-dimensional plot of average processing time ( $A P T$ ) as a function of $F R$ and $C$ with ranges of $85 \%<F R<95 \%$ and $100<C<300$; where $b$ and $\rho$ are kept constant at $0.322(L R=80 \%)$ and 4 respectively .116

Figure 4.18: Plot of $F A P^{*}$ versus $S$ .118 
Figure 4.19: 3-dimensional plot of average processing time ( $A P T$ ) as a function of $s$ and $F A P$ with ranges of $0<s<0.8$ and $0<F A P<5$; where $b$ and $f$ are kept constant at $0.139(L R=80 \%)$ and $0.152(F R=90 \%)$ respectively $(s=0, \rho=4, N=2, C=$ 100)

Figure 4.20: 3-dimensional plot of average processing time ( $A P T$ ) as a function of $s$ and $F A P$ with ranges of $0<s<0.8$ and $0<F A P<5$; where $b$ and $f$ are kept constant at $0.139(L R=80 \%)$ and $0.152(F R=90 \%)$ respectively $(s=0, \rho=4, N=2, C=$ 250)

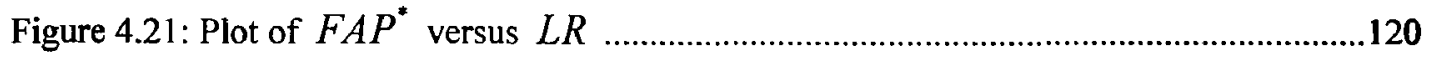

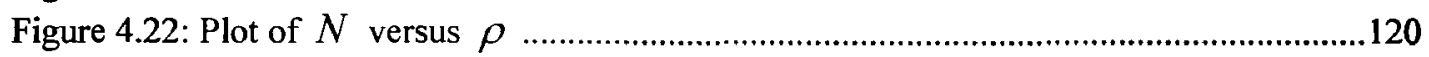

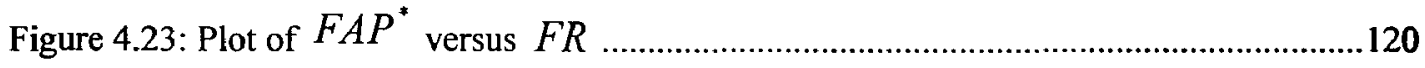

Figure 4.24: Histogram plot of the distribution of $C^{*}$ values...........................................121

Figure 4.25: Histogram plot of the distribution of $F A P^{*}$ values...........................................121

Figure 4.26(a): Plot of $A P T$ versus $C$ ( $s=0, N=2, \rho=5, L R=80 \%, F R=85 \%) .122$

Figure 4.26(b): Plot of $A P T$ versus $C$ ( $s=0, N=2, \rho=2, L R=90 \%, F R=95 \%) 123$

Figure 4.27(a): Plot of $A P T$ versus $F A P$ ( $s=0, N=2, \rho=3, L R=80 \%, F R=95 \%$ )

Figure 4.27(b): Plot of $A P T$ versus $F A P(s=0, N=2, \rho=6, L R=80 \%, F R=$

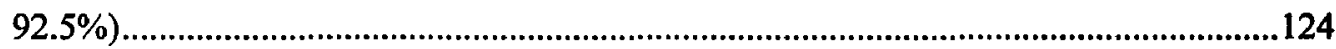

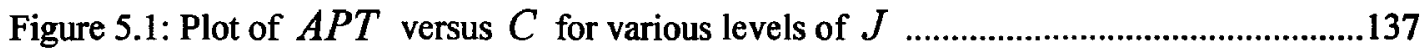


Symbol/Acronym

$A P T$

$A P T_{1}$

$a$

$B$

$b$

$b_{c}$

$b_{m}$

b.

C

$c_{i}$

D

$D_{c}$

$D_{m}$

\section{Definition}

the performance measure of average processing (service) time calculated over approximately 3000 jobs per station

the actual processing time in the first cycle

the job arrival density function

the 'experience' factor

the learning constant (also known as the learning slope)

the learning constant under pure cognitive conditions

the learning constant under pure motor conditions

the combined learning constant (actual learning slope)

the centralization of control policy (the batch transfer quantity)

the number of identical machines at each stage $i$

the time for total forgetting the time for total forgetting for cognitive portions of a task

the time for total forgetting for motor portions of a task 
DPLM

DRC

$d_{i}$

$e_{j i}$

EDD

FAP

FCFS

FISFS

$F R$

$f$

$f^{c}$

$f^{m}$

$f^{*}$

$h$

$J$

$K(n)$ the dual phase learning and forgetting model

the dual phase learning model

dual resource constrained system

the length of the

interruption interval in cycle $i$

the relative efficiency of worker $j$

on stage $i$

the earliest due date job sequencing rule

the upfront training policy (Flexibility $A$ cquisition Policy)

the first come, first served worker assignment rule

the first in system-first served queue discipline rule

the forgetting rate expressed as a percentage $\left(F R=100 \times 2^{-f}\right)$

the forgetting slope

the forgetting slope under purely cognitive conditions

the forgetting slope under purely motor conditions

the combined forgetting slope

the number of machines in the system

product life-cycle measured in jobs per station

the cost of producing the $n$th unit 


\begin{tabular}{|c|c|}
\hline LFCM & the learn-forget curve model \\
\hline LNQ & $\begin{array}{l}\text { the greatest numbers of jobs in } \\
\text { queue worker assignment rule }\end{array}$ \\
\hline$L R$ & $\begin{array}{l}\text { the learning rate expressed as a } \\
\text { percentage }\left(L R=100 \times 2^{-b}\right)\end{array}$ \\
\hline$l$ & $\begin{array}{l}\text { the worker assignment rule (stage } \\
\text { selection procedure in central } \\
\text { control) }\end{array}$ \\
\hline$M$ & $\begin{array}{l}\text { the machine 'non-compressible' } \\
\text { factor }\end{array}$ \\
\hline$m$ & the number of stages in the system \\
\hline$N$ & $\begin{array}{l}\text { the level of worker flexibility } \\
\text { (number of tasks learned) }\end{array}$ \\
\hline$n$ & the number of units (repetitions) \\
\hline$n_{s}$ & $\begin{array}{l}\text { the number of units needed to reach } \\
\text { the standard time of the worker }\end{array}$ \\
\hline$P$ & $\begin{array}{l}\text { the probability of adopting a } \\
\text { certain DRC policy }\end{array}$ \\
\hline$p_{i j}$ & $\begin{array}{l}\text { the job routing transition } \\
\text { probability matrix from stage } i \text { to } \\
\text { stage } j\end{array}$ \\
\hline$q_{i}$ & the queue discipline used in stage $i$ \\
\hline$R$ & $\begin{array}{l}\text { the ratio of time for the first unit } \\
\text { under purely cognitive conditions } \\
\text { to time for the first unit under } \\
\text { purely motor conditions } \\
\left(y_{c}(1) / y_{m}(1)\right)\end{array}$ \\
\hline SOT & $\begin{array}{l}\text { the shortest operation time queue } \\
\text { discipline rule }\end{array}$ \\
\hline
\end{tabular}


the shortest processing time job sequencing rule

the task similarity coefficient used in model B to represent the four levels of task similarity for when $N=3(i=0,1,2,3)$

$s$

$T$

$T_{c}$

$T_{M}$

$T_{M e d}$

$T_{U}$

$t\left(u_{i}+n_{i}\right)$

$u_{i}$

$u_{i}^{c}$

$u_{i}^{m}$

VRIF the task similarity coefficient $(N=2)$

task-type (unspecified)

cognitive task

motor task

median task

uniform task

the time required to process $u_{i}+n_{t}$ units

the amount of experience (expressed in units) retained from the previous $i-1$ cycles

the amount of cognitive experience (expressed in units) retained from the previous $i-1$ cycles

the amount of motor experience (expressed in units) retained from the previous $i-1$ cycles

the variable regression to invariant forgetting model 
the variable regression to variable forgetting model (also referred to as the learn-forget-learn model (LFL))

$\boldsymbol{w}$

the number of workers in the system

WIP

work in process

$y(n)$

the processing time of the $n^{\text {th }}$ unit

$y(1)$

the time required for the first repetition

the intercept of the forgetting curve

$\hat{y}_{i}^{c}(1)$

the intercept of the cognitive forgetting curve

$\hat{y}_{i}^{m}(1)$

$\breve{y}(n)$

$\bar{y}(n)$

$\tilde{y}_{i}(1)$

the intercept of the motor forgetting curve

the total time required to complete $n$ units

the average time to complete each unit if $n$ units are produced

the time to process the first unit in cycle $i$

the time required for the first repetition under purely cognitive conditions

$y_{m}(1)$

the time required for the first repetition under purely motor conditions

$y_{s}$

the processing time standard 
$\begin{array}{ll}y\left(u_{i}+n_{i}\right) & \text { the time needed to acquire } \\ & \left(u_{i}+n_{i}\right) \text { units of experience in } \\ \text { cycle } i\end{array}$

$y\left(u_{i}^{c}+n_{i}\right)$

$y\left(u_{i}^{m}+n_{i}\right)$

$\alpha$

$\alpha_{i}^{c}$

$\alpha_{i}^{m}$

$\phi_{i}$

$\phi_{i}^{c}$

$\phi_{i}^{m}$ the time needed to acquire $\left(u_{i}^{c}+n_{i}\right)$ units of cognitive experience in cycle $i$

the time needed to acquire $\left(u_{i}{ }^{m}+n_{i}\right)$ units of motor experience in cycle $i$

the ratio of total time to forgetting to time needed to acquire $\left(u_{i}+n_{i}\right)$ units uninterruptedly

the ratio of total time to forgetting to time needed to acquire $\left(u_{i}{ }^{c}+n_{i}\right)$ units of cognitive experience uninterruptedly

the ratio of total time to forgetting to time needed to acquire $\left(u_{i}{ }^{m}+n_{i}\right)$ units of motor experience uninterruptedly

the number of units that could have been processing in cycle $i$ if there was no interruption

the number of cognitive units that could have been processing in cycle $i$ if there was no interruption

the number of motor units that could have been processing in cycle $i$ if there was no interruption 


$$
\boldsymbol{\rho}
$$

$\lambda$

$\chi_{i}$

$\pi_{1}$

$\gamma_{i}$ the initial processing time to standard time ratio $\left(y(1) / y_{s}\right)$

the mean job arrival rate

the service rate density function for each machine in stage $i$

the level of centralized control of jobs at stage $i$

the mean service rate of each machine in stage $i$ 


\section{Chapter 1: introduction and literature review}

\subsection{Thesis overview}

This thesis investigation used deterministic simulation models to examine the effect of worker learning and forgetting on dual resource constrained (DRC) shops, facilities with fewer workers than machines. New factors such as job similarity and a variable worker learning rate as a function of the task type were introduced and examined to determine their effect on the shop performance measure of average processing time. It was assumed that the DRC shop under investigation is a static pure-flow shop with deterministic job arrival and processing times with no job queue formation, zero transfer times, and no machine flexibility. The shop consists of a single worker trained for either two or three tasks. It was also assumed that the worker undergoes both learning and forgetting of task experience.

In the first model, model A, the learning-forgetting effects of a worker on the performance of DRC systems was examined. Worker learning and forgetting was modeled according to the dualphase learning-forgetting model (DPLFM). The DPLFM is a model that assumes that a task has separate cognitive and motor requirements. A variable worker learning rate was introduced and was measured as a function of the type of task processed.

In the second model, model $\mathrm{B}$, the assumption of totally different tasks was released to include the processing of tasks with varying degrees of task similarity. The effect of this assumption change on the performance of DRC shops was also examined.

In the third and final model, model $\mathrm{C}$, binary logistic equations were developed that could serve as a management decision making tool for suggesting optimal worker transfer and training policies given less controllable factors such as the worker learning rate, the worker forgetting rate, the level of task similarity, and the ratio of initial processing time to the standard time of 
the job. The method by which these equations could be used as a tool for modifying policies in a pre-existing DRC system was illustrated by a numerical example.

Dual resource constrained (DRC) systems, or multi-skilled flexible workforces, are shops where both machines and labour are limiting resources, namely, $h$-machines and $w$-labourers, with $w<h$ (Wisner and Pearson, 1993). Research on DRC systems is motivated by: (1) increasing labour costs (Naitove, 2003), (2) more widespread Just-in-Time (JIT) implementation (Alternburg et al., 1999), and (3) increased global competition spurred by demands for more customized, make-to-order products (Tompkins, 2002, Karnes and Karnes, 2000).

DRC systems respond to (1) by reducing the size of the labour force by training workers for more than one task, to (2) by reducing bottlenecks at work centers by more frequent worker transfers and by reducing manufacturing lead times, and finally, DRC systems respond to (3) by improving customer service and adapting to frequent changes in product demand (Kher, 2000).

However, the frequent worker transfers common in DRC systems necessitate the relearning of an operation that otherwise might not have been forgotten if the worker was only trained for, and dedicated to, one task. As a result, worker learning and forgetting in DRC shops is an important investigation because it determines, to what degree, how the benefits of a DRC system are overestimated by the detrimental effects of worker learning and forgetting.

This thesis extends upon Jaber et al. (2003) that investigated worker learning and forgetting phenomenon in DRC settings. This thesis also introduces the concept of worker learning and forgetting in a two and three stage DRC system according to the dual-phase learning-forgetting model (DPLFM) developed by Jaber and Kher (2002). This learning and forgetting model is based on the theory that a task has separate cognitive and motor requirements. The effect of including task similarity is also examined. The experiments consist of deterministic simulation models; the results are compared to those of Jaber et al. (2003) and the five possible issues that affect DRC shop performance given by Hottenstein and Bowman (1998).

This thesis is organized as follows. The remainder of chapter I provides a survey of research done in the areas of DRC systems, worker learning theory, learning and forgetting models, and the learning and forgetting phenomenon in DRC systems. Chapter 2 summarizes the development of the dual-phase learning-forgetting model (DPLFM), the learning and forgetting 
model used in model A and model B of this thesis, and illustrates the behaviour of the DPLFM with a numerical example.

Chapter 3 discusses the modeling or experimental designs used in model A, model B, and model $\mathrm{C}$ of this thesis. Chapter 4 provides the results, analysis and conclusions to these models and discusses how they compare to those of Jaber et al. (2003) and the five possible issues that affect DRC shop performance given by Hottenstein and Bowman (1998). Chapter 5 provides thesis conclusions and suggestions for further work in this area.

\subsection{Dual resource constrained (DRC) systems}

Research on scheduling in DRC systems may be easily examined by first outlining the characteristics of the scheduling problem. In this thesis, the production problem falls under the category of short-range production scheduling (Silver et al., 1998). Namely, scheduling that does not require detailed and advanced production planning, but rather, involves individual workstation decision-making initiated by workers or supervisors. Silver et al. (1998) clarified an important distinction when studying short-range production scheduling problems: whether or not the sequence of processing one or more jobs allows for new job arrivals. If the schedule is fixed and no new jobs are expected until each job is processed, then the method is called 'static scheduling'. If the schedule allows for the possibility of new job arrivals the method is called 'dynamic scheduling' (Silver et al., 1998). The work environment in this thesis does not allow for new job arrivals while each job is being processed; and as a result, this thesis only deals with static scheduling. The shop structure also dictates which scheduling approach to use. The simplest shop layout is a pure flow shop. This is where all the jobs must follow the same predetermined sequence through the shop and visit every station. A general flow shop is where the jobs are allowed to skip stations (Silver et al., 1998). In a job shop, the order of jobs on each station may be different for each job and they may be processed in any order. In this thesis, the job routing is known for certain; and as a result, the assumption of deterministic pure flow shop job routing is used. An additional scheduling complication involves parallel machines. In parallel machines, the flow shop or job shop may have more than one machine for processing at any stage. This adds flexibility to the shop structure. In job shop research, the individual work center is referred to as a machine or station, whereas the group of machines with the same operation and processing time is referred to as a stage. In this thesis only individual stations are included in the model. To summarize, the DRC system modeled in this thesis is a static-pure 
flow shop with deterministic job routings, job arrival times, initial job processing times, and, has only one station per stage.

The performance of a shop system is evaluated as various experimental factors are adjusted to determine their effect on one or more performance measure. Sequencing rules are one such experimental factor. Silver et al. (1998) organized sequencing rules into 4 classes: local rules, global rules, static rules, and dynamic rules. Local rules only require information about the queue in question; whereas global rules also require information about work elsewhere in the shop. Static rules are rules based on information that does not change over time such as earliest due date (EDD) rule or shortest processing time (SPT) rule. Dynamic rules rely on information that changes with time such as the minimum slack time rule (Silver et al., 1998). As discussed later, DRC systems can include all of the above types of global and local dispatching rules in addition to 'when' and 'where' each worker should be transferred (Treleven, 1989). Since this thesis examines a static DRC shop model, rules pertaining to station queues are not applicable because no queues are formed. Namely, there is never a job waiting to be processed, or workers waiting to process a task.

Common job shop performance measures are usually classified by their effect on shop congestion or work in process (WIP), and those that are used for meeting due dates (Silver et al., 1998). As previously mentioned, no queues form in this thesis model, and as a result, shop congestion performance measures are not applicable. This thesis uses the shop performance measure of average processing (service) time ( $A P T$ ) calculated over approximately 3000 jobs, the same performance measure used by Jaber et al. (2003).

\subsubsection{Characteristics of a DRC system}

As previously mentioned, the DRC system is a shop where both machines and labour are limiting resources, namely, $h$-machines and $w$-labourers, with $w<h$ (Wisner and Pearson, 1993). The benefits of a DRC shop, commonly referred to as a shop with a multi-skilled flexible workforce, are the reduction of manufacturing lead times (reduction in WIP), improved customer service, and the ability to adapt to frequent changes in product demand (Kher, 2000). The improved customer service is characterized by on-time delivery performance measured by mean tardiness and percent tardy jobs (Park and Bobrowski, 1989). Also, adaptation to frequent demand changes is necessary in a highly competitive work force (Treleven, 1989). Job shops, 
common production settings for DRC systems (Hottenstein and Bowman, 1998), are predominantly make-to-order shops with large WIP and little inventory of finished goods; typical job shop products include printed circuit boards, metal parts, and commercial printers, with each item usually tailored to the wishes of customers (Silver et al., 1998). Rosser (1967) first proposed the concept of dual resource constrained (DRC) work systems. Until that time, only shop problems involving machine-limited resources were examined. The introduction of an additional labour constraint increased the complexity of the shop problem while enhancing upon the flexibility already known in queue discipline rules. Rosser (1967) suggested that the introduction of the labour constraint opened up new levels of design parameters such as the level and quality of the labour force. Most notable was that the quality and level of the workforce were interrelated in DRC systems. This interrelation is described as follows: as the workforce level decreased, the degrees of possible worker flexibility increased, and therefore the quality of the workers increased. Conversely, as the workforce level increased, the amount of possible flexibility decreased, and therefore the quality of the workforce decreased (Rosser, 1967).

\subsubsection{Parameters of a DRC system}

Rosser (1967) clearly outlines the scope (and some possible extensions) of a DRC shop system in his paper "Labour and Machine Limited Production Systems". The DRC system examined in his paper was a stochastic flexible job shop with stochastic job routings. The scope of a DRC system and its design parameters is shown in Figure 1.1.

The notations shown in Figure 1.1 represent workload, design, and control parameters. The workload parameters are as follows: job arrival density function, $a$, mean job arrival rate, $\lambda$, the service rate density function for each machine in stage $i, \chi_{i}$, the mean service rate of each machine in stage $i, \gamma_{i}$, and the job routing transition probability matrix from stage $i$ to stage $j$, $p_{i j}($ Rosser, 1967). The models in this thesis are deterministic, and as a result only the $\lambda$ and $\gamma_{i}$ workload parameters are required to describe the DRC system. The design parameters are as follows: the number of stages in the system, $m$, the number of identical machines at each 


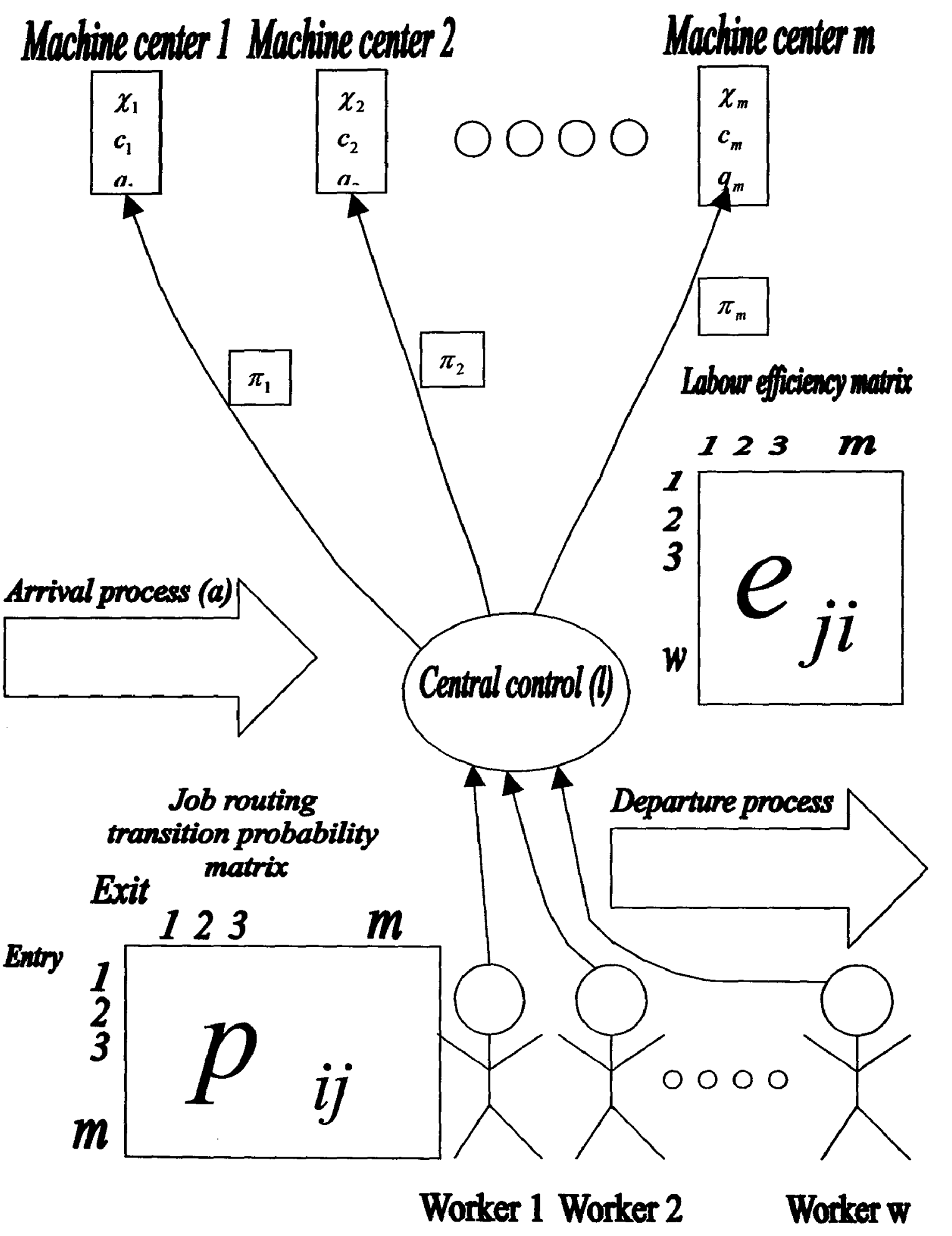

Figure 1.1: DRC generalized model (Rosser, 1967) 
stage $i, c_{i}$, the number of workers in the system, $w$, and the relative efficiency of worker $j$ on stage $i, e_{j i}$. The efficiency parameter is used to determine the service time of the individual at stage $i$. This is done by dividing the service rate for job $k$ on stage $i$ by $e_{j i}, \gamma_{k i} / e_{j i}$, where $e_{j i}$ ranges from 0 to 1 , and $e_{j i}=1$ represents maximum worker efficiency. The central control assigns workers to each stage according to the queue discipline of each stage. In this thesis, it is assumed that each machine at a particular stage has the same processing time distribution $\chi_{i}$. Also, since the models in this thesis are deterministic, only $m, c_{i}$, and $w$ are applicable. In this thesis, $m=2$ or $3, c=1$ for every $i$, and $w=1$. The control parameters are as follows: the stage selection procedure in the central control, $l$, the queue discipline used in stage $i, q_{i}$, and the level of centralized control of jobs at stage $i, \pi_{i}$ (Rosser, 1967). Again, since the models in this thesis are static, and therefore no queues are formed, only $l$ and $\pi_{i}$ are applicable. The level of centralized control represents the extent by which the central control determines when the worker is assigned to the next task. Full control, or when $\pi_{i}=1$, means that central control determines where the worker should go next after the present unit is completed. When $\pi_{i}=0$ means that central control does not allocate the worker to a new station until the worker has completed the work remaining in queue at that station (Hottenstein and Bowman, 1998). In the models of this thesis, the $\pi_{i}$ parameter is neither 0 nor 1 but varies between these two values. Namely, the level of centralized control is determined by the number of units processed as in Jaber et al. (2003). The worker moves from the station after a fixed number of jobs are completed, e.g.: batch sizes of 10,25 , or 250 units. Also, in the thesis, $l$ is assumed to be the next stage in the sequence, i.e. stage1-stage2-stage3.

The common managerial decisions in DRC systems can be grouped into two categories: operating issues and design issues. Operating issues are as follows: when to transfer workers, where to transfer them, queue discipline policies (local, global and dynamic sequencing rules), and job release policies. The 'when' rule is often called the 'centralization of control rule' and the 'where' decision is called 'worker assignment rule' (the aforementioned stage selection procedure in central control, $l$ ). When the control is said to be 'centralized' means that the worker is eligible for a transfer after the present unit is completed $\left(\pi_{i}=1\right)$; 'decentralized' control is when a worker is not eligible for a transfer until the queue at the station is empty $\left(\pi_{i}=0\right)$ (Hottenstein and Bowman, 1998). The queue discipline policy determines the order in 
which the jobs waiting in station queues are processed. The job release policy decides when previously arrived jobs are dispatched to the job floor. Job release policies are classified by either finite loading or infinite loading policies. Finite loading policies consider machine capacities and load jobs to machines according to priorities in order to fill machine capacities, with lower priority jobs postponed until capacity is available. Infinite loading policies, on the other hand, release jobs without considering station capacities, current station loads, or job arrival times. Instead, criteria such as critical ratios (time remaining until due date/total processing time) are used to determine when jobs are released to the shop floor (Wisner and Pearson, 1993). The models in this thesis include the assignment rule, the centralization of control policy (this thesis also refers to this as the worker transfer policy), and job release policy operating issues. In this thesis, the assignment rule is governed simply by transferring the worker to the next station in a simple two or three-stage sequence. The centralization of control policy in this thesis is governed by the worker completing a fixed batch number of units $\left(0<\pi_{i}<1\right)$. The job release policy in this thesis is a finite loading policy where a job is released into the system only after the worker has completed releasing the previous job since the capacity at every machine is only one unit. Therefore no queues are formed, and hence, a queue discipline policy is not applicable.

Design issues are as follows: the degree by which workers are trained and cross-trained, the degree of worker flexibility, labour utilization levels, the routing pattern of jobs, and the manner by which information is collected and used. An important caveat must be considered when discussing flexibility. In DRC literature, labour flexibility may have different meanings such as: the machine staffing level, the level of worker-station efficiency (as previously discussed, $e_{j i}$ ), the level of centralized control $\left(\pi_{i}\right)$, and number of possible stages that a worker can be transferred (Treleven, 1989). In this thesis worker flexibility is defined as the number of stages for which the worker is trained ( $N$ ), a symonym for the degree of worker cross-training. 


\subsubsection{DRC research: an overview}

Two good surveys of DRC research are Treleven (1989) and Hottenstein and Bowman (1998) Several notable conclusions that Treleven (1989) made regarding past DRC research were as follows: the dispatching rules that work well with machine limited systems also tend to work well with DRC systems; DRC systems work most efficiently with a staffing level between $50 \%$ and 75\%; the effectiveness of the 'where' assignment rule is dependent on the level of labour flexibility; the mean and variance of flow time decrease as the control becomes more centralized; the importance of having greater flexibility is heightened as the labour efficiency in the subsequent station is decreased; the relative rankings of decision rules remain the same as the size of the DRC system increases thereby allowing conclusions for larger systems with the simulation of smaller systems; and, performance criteria, such as the ranking of decision rules, are found to be sensitive to high labour utilization levels.

Hottenstein and Bowman (1998) provided a comprehensive survey of DRC research. The research was categorized into five areas of study: worker flexibility, centralization of control, worker assignment rules, queue discipline, and the cost of transferring workers (Hottenstein and Bowman, 1998).

Some of the notable conclusions of their survey concerning worker flexibility were as follows: cross-training beyond two or three skills per worker $(N=2$ or 3$)$ does not significantly enhance DRC performance; the high cost of cross-training further impacts on the cost effectiveness of DRC systems; and, workers need not be perfectly interchangeable as far as their value of $e_{j i}$ is concerned.

Conclusions of their survey concerning centralization of control were as follows: an efficiency control rule that moves a worker as soon as the worker can be moved to a stage that he is most efficient is shown to be a superior rule under most conditions; the degree of centralized control is not independent of the assignment rule; centralized control $\left(\pi_{i}=1\right)$ only marginally reduces mean and variance of flow-time compared to decentralized control $\left(\pi_{i}=0\right)$; and if the efficiency levels of the workers between tasks vary, then the level of centralized control decision

‡ Note that in this section the term 'flexibility' refers to the level of worker cross-training, just as it does throughout this thesis. 
is far less dependent on the status of the queue but is determined by the time of the availability of a station where the worker is more efficient.

Conclusions of their survey concerning worker assignment rules were as follows: the question of where to assign workers has a greater impact on shop performance than when to assign workers; the assignment of workers to stages with queues having the greatest number of jobs (LNQ) had the greatest improvement on the mean and variance of flow-time; the performance of the worker assignment rule is not independent of the queue discipline used at that stage; and, when the $e_{j i}$ of workers vary, they should be assigned to the stages where they are most efficient.

Conclusions of their survey concerning queue discipline rules were as follows: there is an interaction between the effectiveness of the queue discipline rule and the worker assignment rule because the state of the next assigned queue is dependent on the queue discipline used at that queue; the shortest operation time (SOT) in the queue rule results in the smallest mean flow-time and the largest flow-time variance; the first in system-first served (FISFS) rule results in the smallest flow-time variance but the largest mean flow-time; the combination of the SOT queue discipline rule and the LNQ assignment rule results in the lowest mean flow-time; and, the combination of LNQ assignment rule and the FISFS queue discipline results in the lowest queue time variance. It is interesting to note that specific assignment rules can be combined with specific queue discipline rules depending on whether improvements of either mean flow-time or mean variance of flow-time is desired.

Finally, Hottenstein and Bowman (1998) provided conclusions for research on the cost of transferring workers. They are given as follows: overall, increasing the transfer delay hinders shop performance; and, the greater the transfer delay the higher the effectiveness of decentralized control $\left(\pi_{i}=0\right)$. Also, Hottenstein and Bowman (1998) concluded that the effect of learning in setups encountered when transferring a worker to a new station is a relevant transfer cost.

Hottenstein and Bowman (1998) also discussed future research of the implications of variety, teams, and forgetting on DRC systems. The effect of variety assumes the introduction of meandemand changes or new products. The concept of teams involves using a group of individual workers as a base unit instead of one cross-trained worker. Hottenstein and Bowman (1998) suggested that the effect of worker forgetting may cause centralized control to be much more 
effective than decentralized control. He proposed that centralized control would allow the worker to more frequently hone certain skills and thereby reduce the effect of long-term forgetting. The assignment rule would therefore be based on the unique forgetting function of each worker across skill levels; and, the assignment rule would be less dependent on where the worker is more efficient, but rather, where the worker would best relearn in order to prevent further forgetting. In other words, the short-term costs of not adhering to the 'best worker to the best job rule' are offset by the long-term benefits of a more homogenous cross-trained flexible workforce (Hottenstein and Bowman, 1998). Further research on the implications of learning and forgetting in DRC systems are discussed in subsequent sections.

As previously shown, the common performance measures of a DRC system are similar to those of a regular job shop such as mean job flow-time, variance of flow-time and mean number of jobs in the system. The inclusion of a labour constraint has proved beneficial in both increasing the possible areas of job shop research and improving the effectiveness of actual shop performance. For example, it was found that because DRC systems allow for the introduction of cross-trained workers, utilization of labour resources improved during periods of product mix changes and material shortages (Treleven, 1989). Also, DRC research has shown that it is always preferable to acquire flexibility in any degree $(N \geq 2)$ over a strictly machine limited system (where $N=1$ ) (Hottenstein and Bowman, 1998). However, few papers have reported on empirical studies of DRC systems or studies of models of existing systems. As a result, the bulk of the research on DRC systems uses simulation models. This leads to difficulty in analysis because simulation studies often have difficulty interpreting statistically significant differences between mean values of results (Treleven, 1989). However, this thesis uses deterministic simulation modeling as an experimental tool. As a result, the models use deterministic input parameters and therefore statistical analysis of the output parameters is not required. For readers interested in DRC system research, the following papers are also suggested by Hottenstein and Bowman (1998): Bobrowski and Park (1993), Fryer (1973), Fryer (1976), Gunther (1979), Nelson (1967), and Treleven (1987, 1988). 


\subsection{Learning and forgetting models}

\subsubsection{The learning phenomenon - an introduction}

The learning phenomenon has been recognized in industry for many years as a factor that should be considered when determining labour costs. Worker learning must be taken into account when tasks are preformed repetitively so that appropriate labour costs and processing times can be determined. Wright (1936) first proposed that worker learning follows a power function such that the processing time of the worker decreases at a constant rate whenever the amount of units produced is doubled. Wright's learning function is of the form:

$$
y(n)=y(1) n^{-b}
$$

where $y(n)$ is the processing time of the $n^{\text {th }}$ unit, $y(1)$ is the processing time of the first unit, $n$ is the number of units produced so far, and $b$ is the learning slope. The slope is determined as follows:

$$
b=-\frac{\log (L R)}{\log (2)}
$$

where $L R$ is the learning rate, and $0 \% \leq L R \leq 100 \%$. Larger learning rates usually indicate less difficult tasks since there is less opportunity for further learning when tasks are relatively simple. Typical learning rates have been reported to range from as low as $68 \%$ for a difficult task such as 'truck body assembly' to $98.5 \%$ for more tedious tasks such as 'manual grinding'(Konz, 1990). Therefore, the nature of the task performed is a crucial factor when determining appropriate future processing times.

Human learning occurs whenever a task is done repetitively, and may be defined as an increase in performance for successive operations of a task. Often, the improvement between adjacent repetitions is relatively large in the early cycles but becomes small or negligible in later cycles. As a result, in the latter cycles, the improvement can only be measured by improvements in large 
groups of repetitions. Human learning is a phenomenon that we know occurs but are still uncertain about exactly why it occurs (Dar-El, 2000). As for how learning occurs, several authors have proposed their views on this matter. Some of the hypotheses for how learning occurs are as follows: discontinuous movements become smoother, fumbling disappears, greater simultaneity in movements is obtained, and wasted or unsuccessful movements are gradually 'weeded' out (Dar-El, 2000). A previous methods time measurement (MTM) supported this idea by finding that the standard velocity of motions of the worker are achieved early in the learning process, and as a result, supported the idea that learning is not caused by an increased speed of motion but rather a more discriminating choice of movements (Dar-El, 2000). Dar-El (2000) classified human learning research into four broad categories: individual learning, product learning, product development learning, and organisational learning. The learning-forgetting models used in this thesis just pertain to individual learning; this is learning that occurs among individuals, not in groups of people as in the latter three types of learning.

The learning curve models are usually expressed as univariate functions with the dependent variable being the unit processing time or unit cost and the independent variable as the cumulative production or cycle count. Some of the common learning curve models are: the loglinear model, the Stanford-B model, DeJong's learning formula, and the S-curve (Dar-El, 2000).

The power curve in equation (1.1) is the most common and widely applied univariate learning curve function. It is also called the log-linear model. The power curve model can also be given in a cost form as follows (Dar-El, 2000):

$$
K(n)=K(1) n^{-b}
$$

where $K(n)$ is the cost of producing the $n^{\text {th }}$ unit and $K(1)$ is the cost of producing the first unit. The log-linear form of the power model is as follows:

$$
\log [y(n)]=\log [y(1)]-b \times \log (n)
$$


and the learning rate $L R$ (as a percentage) is a function of $b$ :

$$
L R=100 \times 2^{-b}(\%) .
$$

The relationship in (1.5) is because, by definition, the power curve learning model states that, as production doubles, the time to produce the $n^{\text {th }}$ unit decreases at a set rate. For illustration:

Let $\operatorname{production}(1)=n_{1}$, and production $(2)=n_{2}=2 n_{1}$, then substituting into (1.1) gives

$$
\begin{aligned}
& y\left(n_{1}\right)=y(1) n_{1}^{-b} \text { and } y\left(n_{2}\right)=y(1) n_{2}^{-b}=y(1)\left(2 n_{1}\right)^{-b}, \\
& \text { then } \\
& \qquad L R=\frac{y(1)\left(2 n_{1}\right)^{-b}}{y(1)\left(n_{1}\right)^{-b}}=2^{-b} .
\end{aligned}
$$

The graphical form of the power curve and the log-log linear form of the power curve is given in Figure 1.2 and Figure 1.3 respectively. The power curve can be used to find the total time to complete $m$ units $[\breve{y}(m)]$ by assuming that $(1.1)$ is a continuous function; note that the following integration in (1.7) is just an approximation as shown (Dar-El, 2000):

$$
\breve{y}(m)=\sum_{0}^{m} y(1) n_{i}^{-b} \Delta n_{i} \approx \int_{0}^{m} y(1) n^{-b} d n=\frac{y(1)}{1-b} m^{1-b}
$$

then the average time to complete each unit if $m$ units are produced is given as

$$
\bar{y}(m)=\frac{\breve{y}(m)}{m}=\frac{y(1)}{1-b} m^{-b} .
$$

The Stanford B model accounts for the amount of experience at the commencement of the first production cycle by including an 'experience factor' $B$ (Dar-El, 2000). The amount of time to 


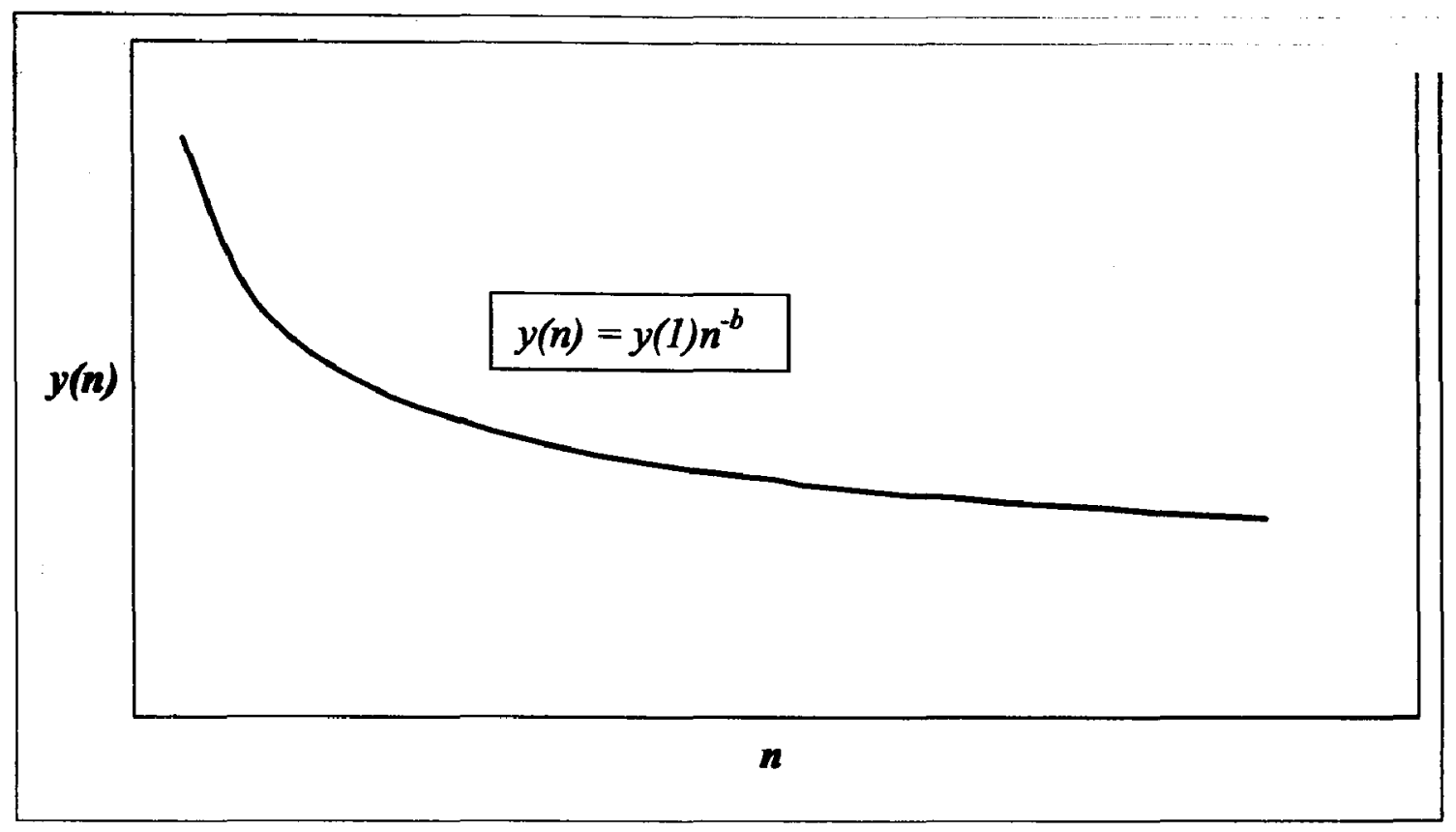

Figure 1.2: Wright's (1936) power learning curve (Dar-El, 2000)

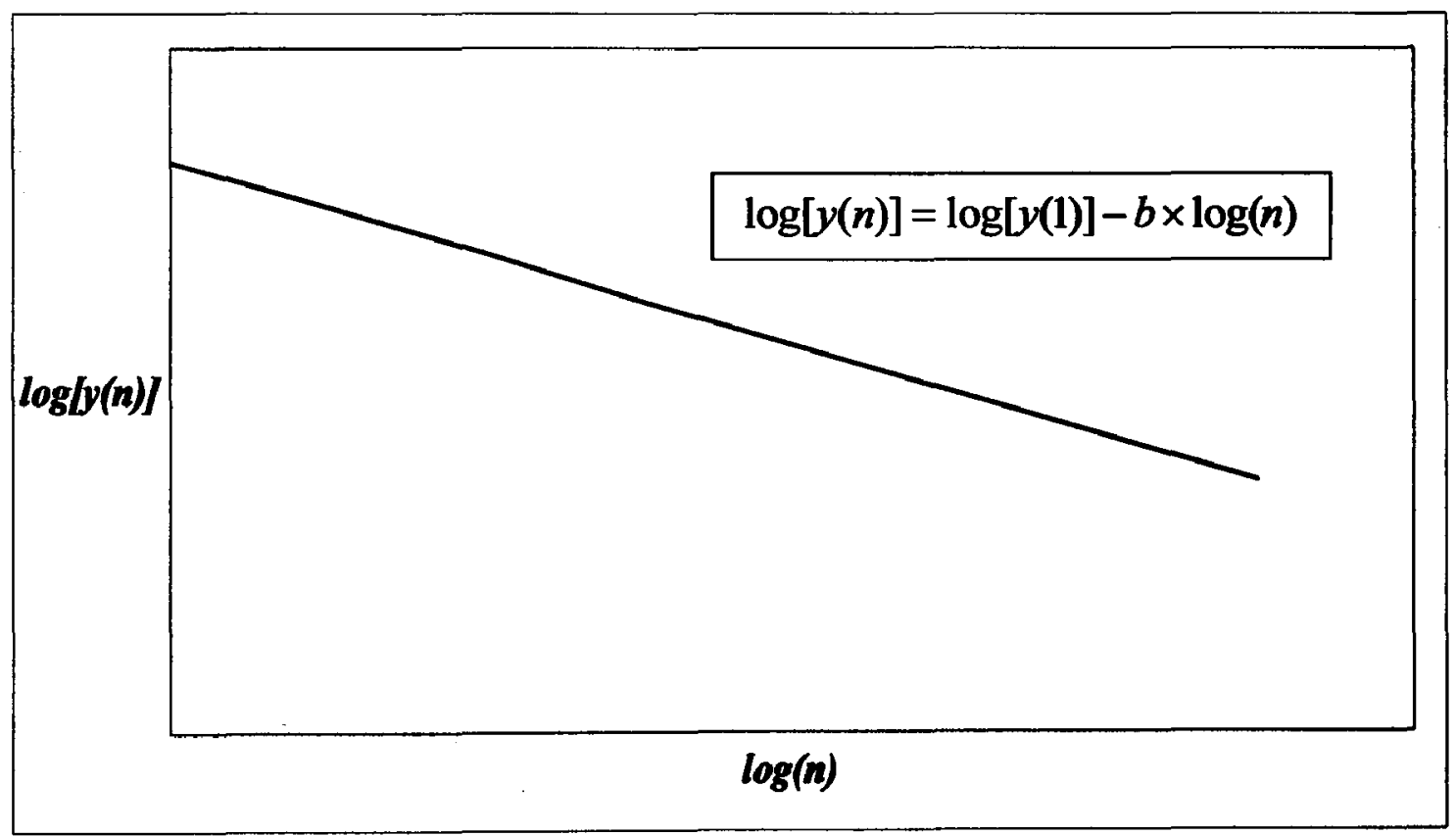

Figure 1.3: Log-log linear form of the power curve (Dar-El, 2000) 
process the $n^{\text {th }}$ unit by the Stanford B model is given by:

$$
y(n)=y(1)(n+B)^{-b}
$$

The Stanford B curve eventually asymptotes to the regular log-linear power model. This effect and the effect of increasing $B$-values are shown in Figure 1.4.

DeJong's learning model was developed to account for sub-components of tasks that do not follow the learning effect such as a machine-dominated portion of a task. A 'non-compressible' factor $M$ is used to account for the machine portion of the task (Dar-El, 2000). DeJong's learning model is given as follows:

$$
y(n)=y(1)\left(M+(1-M) n^{-b}\right)
$$

where $M=0$ is the case of no machine content (model reverts to the original power curve), and $M=1$ is the case where the task is fully automated and no learning is possible. The Stanford B model tends to be more accurate in the early stages, and the DeJong learning model tends to be more accurate in the latter cycles. As a result, the S-curve learning model was developed to take advantage of the characteristics of both the Stanford B model and the DeJong learning model (Jaber, 1996). The S-curve learning model is given as follows:

$$
y(n)=y(1)\left(M+(1-M)(n+B)^{-b}\right)
$$

The behaviour of the above learning models is shown together when the units of output increases by the base of 10 in Figure 1.5. There are many other learning models that have been developed. However, they are not commonly used and they are beyond the scope of this thesis. For interested readers the following papers are suggested: Baloff (1971), Belkaoui (1986), Bohlen and Barany (1976), Buck et al. (1976), Knecht (1974), Lippert (1976), Smith (1989), Steedman (1970), and Teplitz (1991). This thesis uses the DPLFM which is partially based on the learning theory of the dual-phase learning model (DPLM) of (Dar-El et al., 1995). The DPLFM and the DPLM are described in section 2.2 and section 2.1 .1 respectively. 


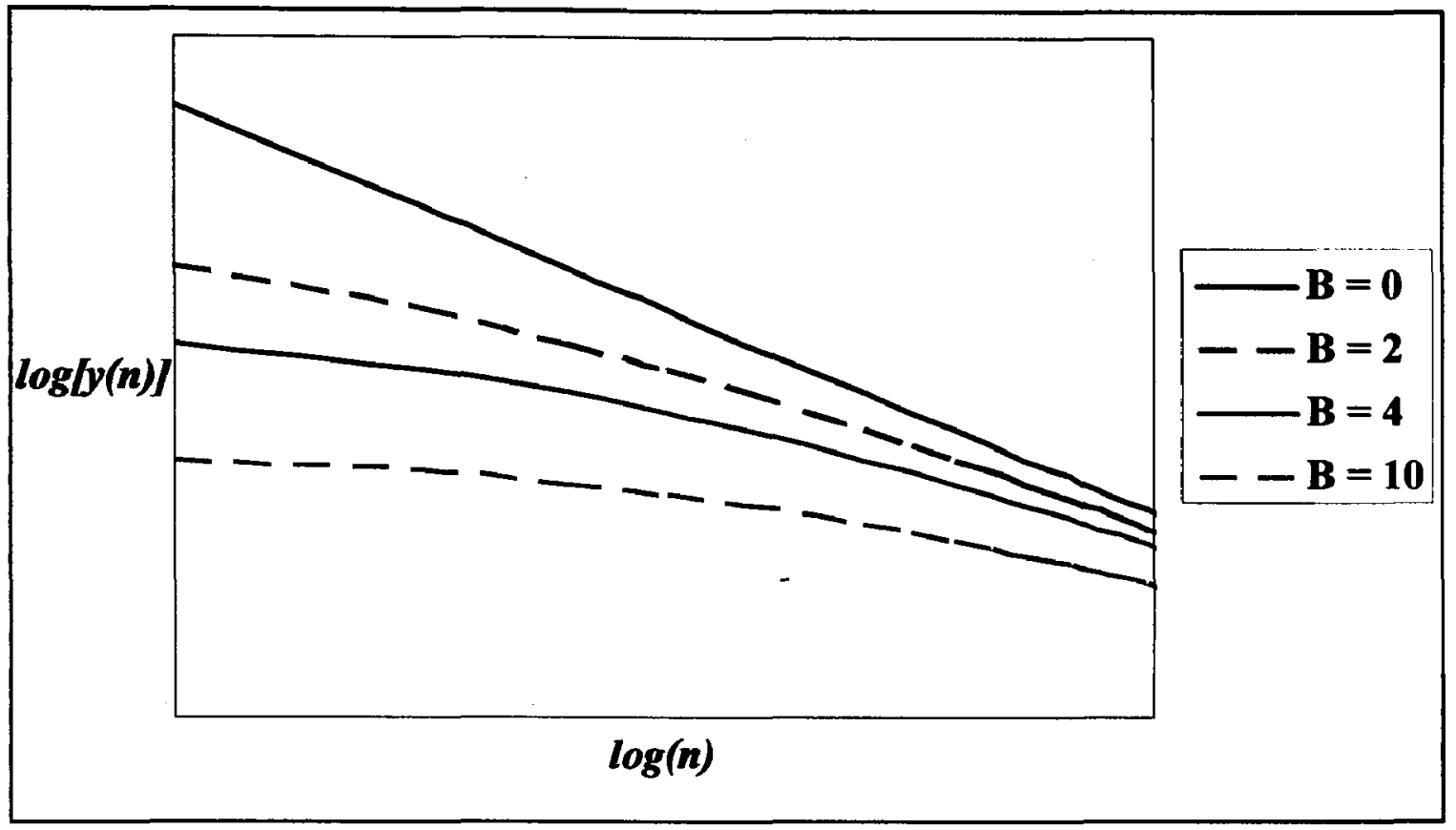

Figure 1.4: The Stanford B model (Dar-El, 2000)

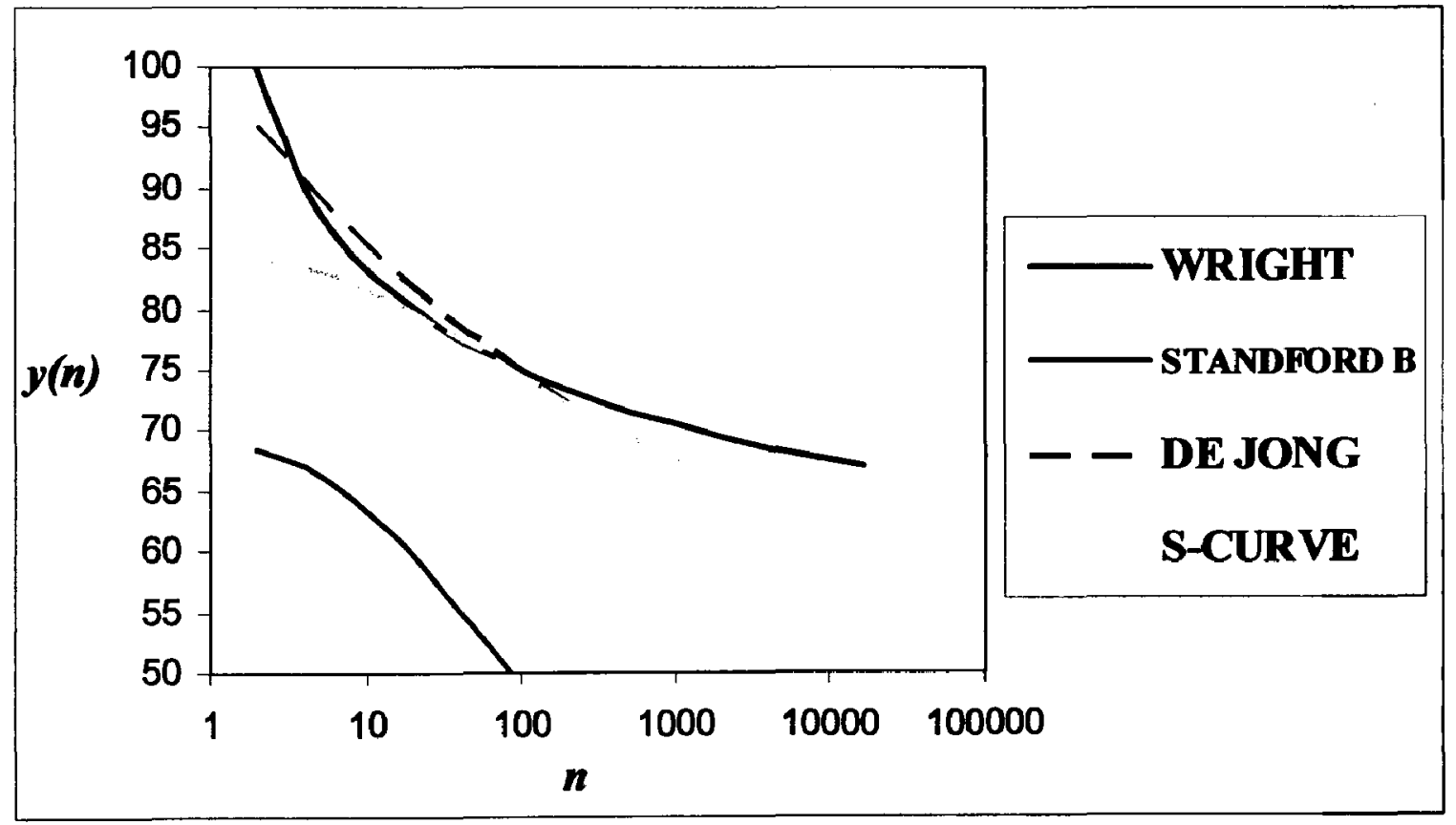

Figure 1.5: The behaviour of common learning models (Jaber, 1996) 
Human learning is influenced by many internal and external factors. Internal factors include the nature of the task being performed or the skill and experience of the worker. Extermal factors include the effectiveness of the ability of the organisation to make workplace improvements such as continuous improvement teams. This thesis only examines internal factors, or those factors that directly relate to pre-established worker and task characteristics. Dar-El (2000) listed 11 factors that effect human learning, they are as follows: methods improvement, worker selection (differences between workers and variations within each operator), previous experience, training, motivation, job complexity, number of repetitions (or cycles), length of the task, errors, continuous improvement, and forgetting. This thesis assumes that the workplace and methods for performing the task are well established beforehand, and as a result, methods improvement is not an experimental factor. Differences between workers are not a factor because the models in this thesis only assume one worker. Also, this thesis assumes that the variation of performance within the worker is negligible. Previous experience is a factor in this thesis because the amount of worker proficiency on the present station is dependent on the experience acquired at predecessor stages. Training is an obvious experimental factor in the models of this thesis because it pertains to the effect of the upfront training policy $(F A P)$. Motivation and worker errors are not addressed in this thesis as experimental factors. Job complexity is not an experimental factor in this thesis as each job is assumed to be equally complex as perceived by the worker. The number of repetitions $\left(n_{s}\right)$ or the time to reach standard time is discussed in experimental design section of chapter 3. Dar-El (2000) explained that the task can in fact be a product of several sub-tasks, some of which may be repeated. In these repeated sub-tasks, the learning rate can be greater than that of the entire task. This is due to the fact that the repeated sub-tasks are being performed more times than the task itself. This phenomenon was categorized as the 'task length' factor. This factor is not addressed in this thesis. The continuous improvement factor is not addressed in this thesis because these models only examine internal learning factors. Finally, forgetting is an experimental design as it is defined and modeled in this thesis by the DPLFM. The importance of the forgetting factor cannot be overlooked if there are interruptions. For example, if there are interruptions in the learning process, the aforementioned learning models do not always provide an appropriate estimation of future processing times. Modern production environments often encounter foreseen or unforeseen work stoppages. For example, the setup of a machine is a task that has foreseen breaks between subsequent setup operations. These foreseen breaks or interruptions are due to the machine processing time between successive setup operations. Also, unforeseen work stoppages such as machine failures, employee related interruptions, or product changes cause interruptions that have a significant effect on the effectiveness of traditional learning curve models. Interruptions cause the deterioration of worker knowledge thorough the phenomena of forgetting. As a result, this lost knowledge has to be eventually relearned in order 
to resume the same level of worker efficiency that was achieved prior to the interruption. For example, Globerson et al. (1998) noted that Anderlohre (1969) found that a plant may lose around $50 \%$ of its productivity due to a production break of three to six months and may lose $75 \%$ of its productivity after a break of one year. Globerson et al. (1998) also noted that McKenna et al. (1985) corroborated Anderholer's findings of performance decay, where they found that there was a decay of $60 \%$ in performance after a break of six months with a total loss of learning after three years. Also, Dar-El (2000) noted that an interruption of a one year can cause a productivity drop of $60 \%$ to $75 \%$. It is important to note that this thesis deals only with forgetting that relates directly to the knowledge lost in individual employees and not other organizational forgetting factors such as employees leaving, changes in products/processes, or lost records or routines. For example, total organisational forgetting caused the cost of producing the Lockheed L-1011 Tri-Star plane to continually rise as cumulative output increased from 1975 to 1982 (Argote and Epple, 1990). As a result, forgetting in this thesis only pertains to individual forgetting and hence is not modeled as potentially severe as total organizational forgetting. Learning and forgetting curves can be applied for needs such as: determining staffing levels, labour costing, production planning, setting time standards, establishing wage incentives, and determining optimal cycle times for assembly (Dar-El, 2000). In this thesis, learning and forgetting models are applied in a multi-function role: (1) in model $\mathbf{A}$, to determine the effect of the task-type factor (variable learning rate) on DRC shop performance using the dual-phase learning-forgetting model (DPLFM) developed by Jaber and Kher (2002) instead of the VRVF or the LFCM to model worker learning and forgetting, and, to determine how this compares with the results of Jaber et al. (2003) where all tasks where assumed equal (fixed learning rate), (2) in model B, to determine how the inclusion of the task similarity factor affects the results of model $A$ and how this compares with the results of Jaber et al. (2003), where the LFCM was used, and, (3) in model $\mathrm{C}$, to construct a multivariate function that provides the optimal upfront training policy $\left(F A P^{*}\right)$ and the optimal centralization of control policy $\left(C^{*}\right)$ (batch transfer frequency) as output variables given four prescribed DRC system factors: task similarity factor $(s)$, initial processing time to standard time ratio of the task $(\rho)$, worker learning rate ( $L R)$, and worker forgetting rate $(F R)$. All of the policy decisions in models $\mathrm{A}, \mathrm{B}$ and $\mathrm{C}$ are examined as they minimize the labour costing performance measure of average processing (service) time ( $A P T$ ).

The following summary of learning-forgetting papers illustrate that worker relearning is costly and is not accounted for in traditional learning curve models such as Wright's power curve model. 


\subsubsection{Learning and forgetting research: an overview}

The costs of forgetting become an important factor to consider when analyzing work environments. As a result, worker-forgetting research was appended and integrated into traditional learning theories. Research on industrial learning and/or learning and forgetting can be grouped into two categories: (1) those papers that perform an experiment and then validate the results statistically with partial or no theoretical validation; and (2) those that develop a theoretical (mathematical) model that describes the learning-forgetting phenomena with partial or no empirical or experimental validation. The following summarizes predominantly experimental papers in learning-forgetting research, or type (1) papers.

Globerson et al. (1989) experimentally derived a forgetting function that allows for the calculation of processing time lost due to an interruption. A specific power model consisting of two parameters and the break time between sets was fitted to the experimental results. This model was then combined with the Stanford B model to obtain a function that determined the relative magnitude of forgetting as a function of the break length and the performance time prior to the break. Bailey (1989) experimentally tested five hypotheses on the nature of learning and forgetting and subsequent relearning. The three most notable hypotheses were: (1) forgetting of a 'continuous' task is negligible; (2) the learning rate is not correlated with the forgetting rate; and, (3) the relearning rate for an assembly task is a function of the original learning rate. The experiment consisted of testing 31 subjects with a 'procedural' and 'continuous' task. A procedural task was defined as one that consists of discrete motor responses such as a car repair job, and a continuous task was defined as consisting of repetitive movements with no clear beginning or end such as riding a bicycle. His experiment used the assembly and disassembly of an Erector set toy, which corresponded to a procedural and continuous task respectively. It was found that hypotheses (1) and (2) were true and hypothesis (3) was false.

The aforementioned experimental research effectively draws conclusions about the behaviour of industrial learning and forgetting. However, they fail to distinguish between the learning and forgetting of simple tasks versus those of complex tasks.

The notion of simple versus complex tasks was highlighted by Bailey (1989) when he distinguished between 'procedural' and 'complex' tasks respectively. However, task distinction may be more explicitly described when tasks are classified as either 'cognitive' or 'manual' (motor) tasks, suggesting that task difficulty is a function of the elements of cognitive and manual components of the task. This distinction was suggested in the experimental paper by 
Sparks and Yearout (1990). In their paper, an experiment was conducted in order to determine if there is a difference between the forgetting functions of manual versus cognitive tasks. The experiment used 16 subjects, eight of whom were tested on a manual peg-board task and eight of whom were tested on a cognitive computer game task on a computer display monitor. The subjects were tested again after a 28 -day break period. The time to reach steady state and the learning curve results were obtained for before and after the break period for the manual task subjects, the cognitive task subjects, and a control group who maintained a certain level of practice during the break period. The results concluded that the learning curve rates before the break were $91 \%$ and $95 \%$ for the cognitive and manual task respectively. The learning curve rates after the break were $94.5 \%$ and $99.15 \%$ for the cognitive and manual task respectively. Steady state was reached almost immediately following the break for the manual task, whereas it took an additional 33 iterations for the cognitive task. These results implied that forgetting for a manual task was negligible as compared to that of a cognitive task. It was suggested that this was an important conclusion because of the increasing amount of cognitive tasks found in modern manufacturing environments. Hewitt el al. (1992) conducted an experiment to determine if worker-relearning rates were different than learning rates after an interruption that ranged between two and 83 days. The experiment used a low-cognitive pegboard task and a moderately high cognitive computer graph/spreadsheet task. This experiment was modeled after the dual motor and cognitive study of Sparks and Yearout (1990). The time-lost-to-forgetting results obtained in the experiment were compared to results of Globerson et al. (1989), where the learning and relearning rates were assumed equal. The results of their experiment concluded that assuming that the learning and relearning rates are equal may cause overly conservative task times after an interruption and thereby may result in excessive worker idle time and worker utilization.

The cognitive and motor elements described in the above papers were either just implicitly described or just included as a synonym for difficult and simple tasks. Also, what was lacking was an explicit mathematical model of how these cognitive and motor elements interact with the learning phenomena. Dar-El et al. (1995) developed such a model and referred to it as the dualphase learning model (DPLM). This model was the first to account for cognitive and motor elements in a learning model that was validated experimentally. Their paper could be classified as both a theoretical and an experimental paper, or as a type (1) and type (2) paper. The 'dual' term implied the dual cognitive and motor nature of human learning. Their study suggested that the learning slope is a variable, and that it gradually reduces as experience is gained. It is hypothesized that this reduction is due to the dual cognitive and motor aspects of learning inherent in the learning slope; namely, the learning slope $b$, actually consists of both a cognitive 
element $b_{c}$ and a motor element $b_{m}$. In this way, Dar-El et al. (1995) suggested that Wright's learning model (Wright, 1936) implicitly included both cognitive and motor elements. During the early task repetitions it is reasoned that the individual retrieves information from the longterm memory. As a result, the early stages are dominated by cognitive elements of learning. As experienced is gained, and as less long-term knowledge is required, the motor element dominates the task. This hypothesis is tested experimentally using one task that is highly cognitive and one task that requires mainly motor skills. The following summarizes predominantly theoretical papers in learning-forgetting research, or type (2) papers.

Carlson and Rowe (1976) addressed the cost of worker forgetting. This paper proposed the use of a $\log$-linear and cubic learning curve as a means of determining the relative effect of an interruption in the learning process. The use of the cubic curve was validated both experimentally in this paper and empirically using historical data. It was assumed that the forgetting portion of the learning curve could be modeled as a negative decay function comparable to electrical losses in condensers. However, they provided no empirical evidence for this assumption. It was suggested that the cubic model is a more accurate representation of the learning process because it is derived from a natural 'S-shape' learning process. A learn-forget model was developed based on this shape. This model was later referred to as the 'variable regression to variable forgetting model' or the VRVF model (Jaber and Bonney, 1997).

Jaber and Bonney (1996) developed another learn-forget model by mathematically determining the forgetting slope if the amount of units of production (up to the point of interruption), the learning rate, and the time to total forgetting is known. This model is called the learn-forget curve model (LFCM). The accuracy of this model is empirically verified using the experimental results of the forgetting phenomena in Globerson et al. (1989), where the Stanford B model was used (Jaber and Bonney, 1997).

In a theoretical comparative study by Jaber and Bonney (1997), three learn-forget curve models (VRIF, VRVF, and LFCM) were tested against two hypotheses deemed important to learning and forgetting phenomena, namely: (1) in the case of total forgetting, the curve asymptotes to a unique value equivalent to the time to produce the first unit with no prior experience; and, (2) the intersection point of interruption is equal on both the learning and forgetting curve (Jaber and Bonney, 1997). Their study showed that the VRIF satisfied the first hypothesis but violated the second, the VRVF violated the first hypothesis but satisfied the second, and the LFCM satisfied both hypotheses. The unique characteristic of the LFCM was that in addition to calculating a unique intersection 
point every interruption-cycle like in the VRVF, the LFCM also recalculated a unique forgetting curve slope $f$. The LFCM model accuracy was validated empirically using historical experimental data (Jaber and Bonney, 1997).

Jaber and Kher (2002) took this dual nature of learning one step further and accounted for the forgetting effect by combining the dual-phase learning model (DPLM) of Dar-El et al, (1995) with the LFCM developed by Jaber and Bonney (1996). This resulted in the dual-phase learningforgetting model or the DPLFM. The DPLFM combined the dual cognitive-motor aspects of learning of the DPLM with the variable forgetting slope function of the LFCM. The DPLFM was then able to determine the level of forgetting that occurs when the forgetting rate, the length of interruption, and the level of experience gained prior to the point of interruption is known Jaber and Kher (2002). The inclusion of the dual nature of learning allowed for the variation in learning and forgetting when the specific cognitive and motor contents of the task are taken into account.

Shafer et al. (2001) examined the effect of learning and forgetting on assembly line performance. In their paper, a simulation experiment was conducted in order to find if there was a statistically significant difference in shop performance when workers are modeled as having homogeneous learning and forgetting distributions as compared to having heterogeneous distributions. Contrary to intuition, the results of the experiment suggested that the productively of the workforce increases as the variability of the learning and forgetting parameters of the workers increase. In other words, the productivity of the system is greater if workers are modeled as having unique learning-forgetting distributions as compared to assuming a fixed distribution across workers.

This thesis examines the effect of learning and forgetting on DRC systems, more specifically, worker learning and forgetting that resembles that of the DPLFM of Jaber and Kher (2002). The next section highlights research on learning and forgetting in DRC research. 


\subsection{Learning and forgetting in DRC systems: an overview}

Worker transfers result in interruptions in the learning process of the worker. These interruptions cause forgetting losses that affect the benefits of using DRC systems. For example, when a worker is transferred to a new workstation without adequate training on the present station, he/she experiences significant forgetting losses after returning to the original workstation. These forgetting losses can be mitigated by providing a worker with upfront training and/or determining the optimal level of centralized control or assignment policies. As a result, the issue of determining the optimal trade-off between acquiring flexibility and reducing forgetting losses in a DRC system is relevant and complex.

The effect of worker learning and forgetting may change the assumptions and conclusions made in traditional DRC systems research as summarized by Hottenstein and Bowman (1998) and Treleven (1989). Again, as in the previous learning and forgetting research summary, this section is divided into: (1) those papers that perform an experiment and then validate the results statistically with partial or no theoretical validation; and, (2) those that develop a theoretical (mathematical) model that describes the learning-forgetting phenomena in DRC systems with partial or no experimental validation. A review of learning and forgetting research in DRC systems begins with the former type (1) research paper.

Wisner and Pearson (1993) were the first to address the notion that relearning losses in DRC systems may affect previously studied DRC performance measures. Their paper is classified as an empirical simulation study because many of the input parameters are empirically validated by an actual DRC shop environment such as: mean processing time, job flow time, tardy costs, labour costs, and labour utilizations rates. Wisner and Pearson (1993) assumed that when a worker is transferred a relearning loss is incurred. However, even though it was implied that relearning involved task-forgetting, the relearning was not time dependent and therefore the concept of worker forgetting was not explicitly addressed in their paper. Their full factorial simulation experiment concluded that relearning losses significantly affect traditional DRC performance measures and that the notion of worker forgetting should be considered whenever DRC systems are studied or managed. The remaining papers are all of the theoretical/mathematical type. The following summarizes predominantly theoretical papers in learning-forgetting research in DRC systems, or type (2) papers. 
Malhotra et al. (1993) were the first to examine the detrimental effects of learning in a DRC job shop. Their paper used a mathematical simulation model where the results were statistically validated, and the inputs and results were partially validated empirically. Previous work that just incorporated the factor of worker flexibility was extended upon in their paper to include the effects of worker learning and attrition rates. The degree by which performance measures such as mean flow time, mean tardiness, and percentage of jobs tardy differed from those of the base case (of just job flexibility) was examined. However, their paper did not assume any costs of relearning due to worker forgetting. An interesting finding of their study was that, in the presence of high learning losses, cross-training workers in more than three departments worsens system performance. Kher et al. (1999) were the first to address both learning and forgetting effects in a DRC system. Their paper utilized mathematical modeling with partial empirical validation of inputs and results. Statistical validation of results was not necessary because of deterministic input parameters. Their paper integrated the worker forgetting effects of the learnforget-learn (LFL) (referred to by Elmaghraby (1990) as the VRVF) model developed by Carlson and Rowe (1976) into a DRC system context. The experimental factors considered by Kher et al. (1999) were as follows: (1) the degree of the upfront training policy ( FAP -0 ), $(F A P-1),(F A P-2)$, (2) the level of worker flexibility, $(N=2,3)^{\S}$, (3) the forgetting rate $(85 \%$ or $95 \%)$, (4) the attrition rate/training period ratio $(2,4,8,12,16,20,40)$, with the attrition rate being a random variable, and, (5) three batch size transfer intervals of $(10,25,250)$. The FAP $-0, F A P-1$, and $F A P-2$ upfront training policies meant that the training period of the worker was complete after the first batch, after the 'standard time' was reached, or after two times the 'standard time' was reached. The centralization of control rule $(C)$ was governed by the completion of batches and was tested for the above three batch sizes. The two performance measures tested were the attrition adjusted average processing time, and the final level of efficiency defined by standard time divided by the last unit processed. The results of their paper concluded that in the presence of high forgetting and attritions rates, workers do not even achieve their standard processing time efficiency (Kher et al., 1999). Also, only in situations of extensive initial training and large batch sizes is a worker flexibility of three a feasible option with respect to average processing time values (Kher et al., 1999). This limits the benefits of flexibility beyond two in DRC systems of significant learning and relearning losses (Kher et al., 1999).

McCreery and Krajewski (1999) were the first to test the effects of task variety and task complexity on the performance of a DRC system with learning and forgetting effects. Their

$\$$ Note that a maximum of three workstations is used because of the Malhotra et al. (1993) finding that cross-training workers in more than three departments worsens system performance. 
paper utilized a mathematical simulation model where the results were statistically validated and the input parameters were partially validated experimentally from field data and empirical studies. There was no empirical validation of results. Their simulation experiment answered the following two hypotheses: (1) there is no difference between different levels of cross training given to workers, and; (2) there is no performance difference between different worker deployment policies. The model consisted of a U-shaped assembly configuration with 12 stages, and 12 workers with two stations per stage. The type of work described in McCreery and Krajewski (1999) consisted of assembly operations where workers were allowed to transfer between stations according to whether they were 'fixed' or 'floating'. Fixed workers were designated a 'home' station, and only left this station when the queue was empty. Workers could visit another station that they were additionally trained for but must immediately return to their home station as soon as there was work available. However, a floating worker would move to another station they are additionally trained for with the longest queue after the completion of every unit. Their paper assumed a worker learning curve according to the DeJong (1957) model. However, their paper did not use a power forgetting curve rate but rather assumed a linear progress back up the original learning curve during an interruption, and, that total forgetting occurred according to the difficulty of the task, with a complex task requiring 30 days for total forgetting and a simple task requiring 60 days. As a result, the forgetting function was linear with respect to the interruption interval. The experiment consisted of four experimental factors and eight fixed factors. The experimental factors were: product variety, task complexity, degree of cross-training, and proportion of fixed workers. The product variety factor consisted of four sub-factors: number of products in product line, task-time variability within product types, variability in product routings, and rate of product turnover. The task complexity factor also consisted of four sub-factors: learning rate, proportion of learning possible, predominant type of learning, and speed of forgetting. The proportion of learning possible measured the fraction of standard processing time to initial processing time. This fraction varied between the 'home' and 'away' tasks of the workers. The predominant type of learning sub-factor included two levels: 'process' learning and 'product' learning. Process learning pertained to generic skills that were transferable to any product that required those tasks. As a result, the interruption interval just included the elapsed time since this generic task was last performed. Product learning, on the other hand, specified that the task is different across products. Therefore, the interruption interval included the elapsed time since this unique product-process task was performed. The effect of these factors on the performance measures of product throughput, work in process, and worker utilization was examined by analyzing the results of four work environment settings: low task complexity-low product variety, low task complexity-high product variety, high task complexity-low product variety, and high task complexity-high variety. The results of their simulation experiment concluded that performance in the first environment is best with just a 
little cross-training; however, high levels of cross-training do little good. In the second environment, high levels of cross-training and flexible worker deployment are the best policy. In fact, the second level environment benefited most from worker cross-training. In the third environment, a little cross-training and restricted deployment is best. In the fourth environment, high cross-training and restrictive deployment was the best policy.

In Kher (2000), a stochastic version of the study done by Kher et al. (1999) was conducted. His study utilized a mathematical simulation model where the results were statistically validated, and there was partial empirical validation of inputs and results. Also, the same learning-forgetting model (LFL), upfront training policies, attrition rate/training period ratios, and forgetting rates $(85 \%, 90 \%, 95 \%)$ as Kher et al. (1999) were assumed, with the last two factors varying in quantity only. However, in Kher (2000), the DRC system was modeled as stochastic with exponentially distributed job arrivals and processing times. The number of operations per job was also random and uniformly distributed. The DRC system consists of six departments, each containing four machines and two workers, resulting in a staffing level of $50 \%$. The centralization of control rule was decentralized and the worker assignment rule was first come, first served (FCFS). The shop performance measures used were mean flow time and mean tardiness. A warm-up period of three years was used prior to the collection of the simulation results over a subsequent period of 15 simulated years. A base-case of no flexibility was used for comparison. The results of the simulation were as follows: DRC systems with forgetting rates of $90 \%$ and $95 \%$ are always preferable to the base case with respect to both performance measures; worker flexibility when forgetting rates are $85 \%$ is only preferable to the base case when the attrition rate is lowest; incremental flexibility can overcome the detrimental effects of worker attrition in the $90 \%$ - 95\% forgetting range; and, $F A P-1$ and $F A P-2$ reduce relearning losses but do not help to improve the two performance measures in the $90 \%-95 \%$ forgetting range.

Jaber et al. (2003) used mathematical modeling in a deterministic simulation where there was partial empirical validation of inputs and results. Statistical validation of results was not necessary because of deterministic input parameters. They extended upon the findings of Kher (2000) by incorporating the LFCM of learning and forgetting of Jaber and Bonney (1996) instead of the VRVF model of Carlson and Rowe (1976). Their choice of the LFCM over other models was justified by its ability to satisfy what was stated as the seven characteristics of learning and forgetting. These characteristics were obtained through past studies of learning and forgetting and were listed as follows (Jaber et al., 2003): (1) the amount of experience gained before interruption occurs in the learning process influences the level of forgetting; (2) the length of the interruption 
interval influences the level of forgetting; (3) the relearning rate is the same as the original learning rate; (4) the power-based model is appropriate for capturing forgetting effects; (5) learning and forgetting are mirror images of each other; (6) the level of forgetting depends on the rate at which a worker learns; and, (7) the nature of the task being performed influences the amount of forgetting. The VRVF model used by Kher (2000) was shown to underestimate the amount of forgetting in Jaber and Bonney (1996). As a result, Jaber et al. (2003) used the LFCM in a similar but deterministic experimental design as Kher (2000). The results of Jaber et al. (2003) were as expected: the VRVF underestimated the amount of total forgetting over subsequent cycles because the VRVF does not recalculate a unique forgetting curve slope every cycle as does the LFCM. The conclusions of Kher (2000) stated that no upfront training $(F A P-0)$ is the best way to improve shop performance under severe forgetting $(85 \%)$. The results of Jaber et al. (2003) suggest that this conclusion had to be further justified since the VRVF may mislead managers by providing shorter estimates of time standards. The results for $F A P-1$ and $F A P-2$ concur with the findings of Kher (2000) when the worker learns two tasks. However, when the worker learns three tasks, the VRVF overestimates forgetting losses due to the absence of the adjustment of the forgetting rates of subsequent cycles. In addition, Kher (2000) stated that upfront training did not improve worker performance. The results of Jaber et al. (2003) suggest that this also may be unsubstantiated because the VRVF model does not carry over the positive affects of training through subsequent cycles, as does the variable forgetting slope of the LFCM.

Jaber et al. (2003) also organized their analysis by posing three specific questions: (1) Will providing upfront training reduce forgetting? (2) Does the frequency of worker transfers relate to forgetting? (3) Do the answers to (1) and (2) change if the amount of training or forgetting rates is changed? The notable results of the three questions were as follows: increasing training to $F A P-1$ or $F A P-2$ always reduces forgetting losses, varying the forgetting rate and the number of tasks learned affects the impact of the worker transfer frequency $(C)$ and upfront training ( $F A P$ ) on forgetting losses, and increasing the rate of worker transfers reduces the forgetting losses. Interestingly, most notable was the unexpected degree of interaction between the transfer and training polices. For example, either increasing the transfer policy or introducing initial training reduces forgetting losses when forgetting rates are low and there is a transfer policy of 10 units $(C=10)$. It is not necessary to increase both the training and transfer policy. This phenomenon is observed until the case of when three tasks are learned $(N=3)$ and forgetting rates are high. It is not until this point that both increasing the transfer policy and the amount of training is needed to reduce forgetting effects. 
Jaber et al. (2003) also included the effect of the degree of job similarity in the LFCM model. Their extension to the LFCM model allowed for a degree of similarity between two or three jobs over the original LFCM assumption of no job similarities. The effect of job similarity caused the relative importance of initial training and transfers to decline with increasing levels of job similarity (Jaber et al., 2003). This thesis has the same experimental design structure of Jaber et al. (2003) with respect to DRC system formulation and task similarity factors. However, this thesis extends upon the work of Jaber et al. (2003) by examining the effect of a variable worker learning rate on the performance of DRC shops by introducing different task-types with respect to the degree of cognitive and motor elements of the task. This dual nature of learning in DRC systems is modeled using the dual-phase learning-forgetting model (DPLFM) developed by Jaber and Kher (2002). This model is hereafter referred to as model A. Then a second model incorporates the new job similarity factor into model $A$ and examines how this compares with the results of Jaber et al. (2003), where the learn forget curve model (LFCM) was used. This model is hereafter referred to as model $B$. Then a third model develops a multivariate function that provides the optimal upfront training policy $(F A P)$ and the optimal centralization of

control policy $\left(C^{*}\right)$ (batch transfer frequency) as output variables given four prescribed DRC system factors: task similarity factor $(s)$, initial processing time to standard time ratio of the task $(\rho)$, worker learning rate ( $L R$ ), and worker forgetting rate $(F R)$. This model is hereafter referred to as model $\mathrm{C}$. All of the policy decisions in models A, B and C are examined as they minimize the labour costing performance measure of average processing (service) time ( $A P T$ ) calculated over approximately 3000 jobs, the same performance measure used by Jaber et al. (2003). A numerical example is included to illustrate the practicality of the function developed in model $\mathrm{C}$. In addition, all of the results of the aforementioned models are compared to the results of Jaber et al. (2003) and the five possible issues that affect DRC shop performance given by Hottenstein and Bowman (1998).

\subsection{Chapter 1 summary}

The previous chapter 1 has provided a survey of DRC systems research, briefly outlined worker learning theory, described some pertinent worker learning and forgetting models, and has reviewed research on the learning and forgetting phenomenon in DRC systems. The following chapter 2 summarizes the development of the dual-phase learning-forgetting model (DPLFM), the learning and forgetting model used in model A and model B of this thesis. Chapter 2 concludes by illustrating the behaviour of the DPLFM with a numerical example. 


\section{Chapter 2: the dual-phase learning- forgetting model (DPLFM)}

\subsection{Prologue}

In modern industrial settings, workers perform tasks that have various levels of mental and manual requirements. Also, frequent interruptions and shorter production runs cause workers to forget previously gained task experience. Traditional learning curves such as Wright's (Wright, 1936) power model do not capture these two characteristics of worker learning in industrial settings. The dual-phase learning-forgetting model or the DPLFM (Jaber and Kher, 2002) is the first to model both the cognitive and motor elements of learning, and learning and forgetting losses due to interruptions. Jaber and Kher (2002) did this by integrating the dual-phase learning model (DPLM) of Dar-El et al. (1995) with the learn-forget curve model (LFCM) of Jaber and Bonney (1996) to develop the DPLFM.

As previously discussed, the DPLM was the first model to account for cognitive and motor elements in a learning model that was validated experimentally. Also, the LFCM is the only empirically validated mathematical model of learning and forgetting that contains two important features of learning and forgetting curves: it contains a forgetting curve that asymptotes to a unique value equivalent to the time to produce the first unit with no prior experience and, it has an intersection point of interruption equal on both the learning and forgetting curves (Jaber and Bonney, 1996). The LFCM does this by re-calculating a unique forgetting curve slope after each interruption.

The unique features of both models were combined to formulate the DPLFM. New mathematical qualities inherent to the DPLFM resulted. These qualities are used to model the seven characteristics of learning and forgetting that have been summarized in industrial learning and forgetting research (Jaber et al., 2003). These characteristics are given by Jaber et al. (2003) and are listed as follows: (1) the amount of experience gained before an interruption occurs in the learning process influences the level of forgetting; (2) the length of the interruption interval influences the level of forgetting; (3) the relearning rate is the same as the original learning rate; (4) the power-based model is appropriate for capturing forgetting effects; (5) learning and forgetting are mirror images of each other; (6) the level of forgetting depends upon the rate at 
which a worker learns; (7) the nature of a task being performed influences the amount of forgetting.

The DPLFM learning and forgetting model most closely embodies the above characteristics, and accounts for cognitive and motor elements in a learning model that was validated experimentally (Jaber et al., 2003); and as a result, the DPFLM was chosen to simulate the worker learning and forgetting effect in the DRC systems modeled in this thesis.

\subsubsection{The dual-phase learning model (DPLM)}

The dual-phase learning model (DPLM) (Dar-El et al., 1995) was developed in order to model two main learning phenomena found in experimental settings, namely: that cognitive and motor learning occurs at different rates, and, these rates vary with time. It was also developed to account for prediction errors in carefully researched empirical data (Dar-El, 2000). When the empirical data were taken in the early stages of the learning curve, traditional learning curve models underestimated future processing times, see Figure 2.1 (Dar-El et al., 1995). Also, when the estimation of initial processing times were predicted from later empirical data, the early cycle estimates underestimated actual processing times, see Figure 2.2 (Dar-El et al., 1995). It was found that tasks that involve both cognitive and motor learning have a faster learning rate than tasks with mainly motor learning; and that cognitive-motor tasks have a larger learning constant in the initial learning stages than the later stages (Dar-El et al., 1995). These two finding imply that cognitive tasks have a larger learning rate than motor tasks and that the cognitive portion of the task is most prevalent in the early stages, whereas the motor element dominates the latter stages, see Figure 2.3. This dual-learning phenomenon explained the aforementioned empirical prediction errors of traditional learning models. These findings led to the development of the DPLM which included a variable learning slope as a function of both the cognitive and motor learning rates. The behaviour of this variable learning rate is shown in Figure 2.3. 


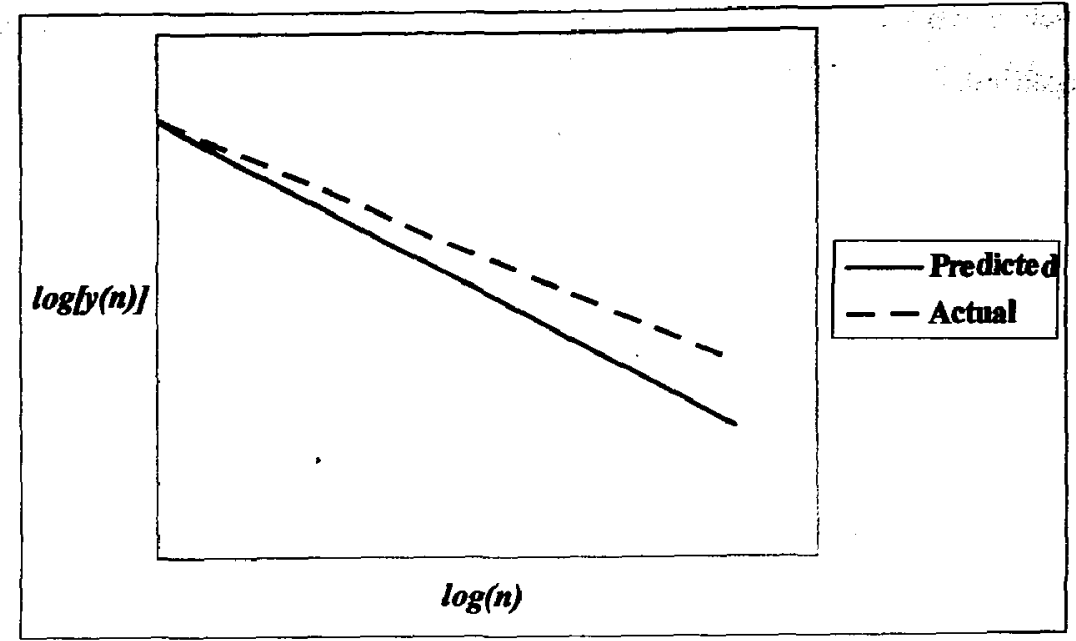

Figure 2.1: Prediction based on early data (Dat-El, 2000)

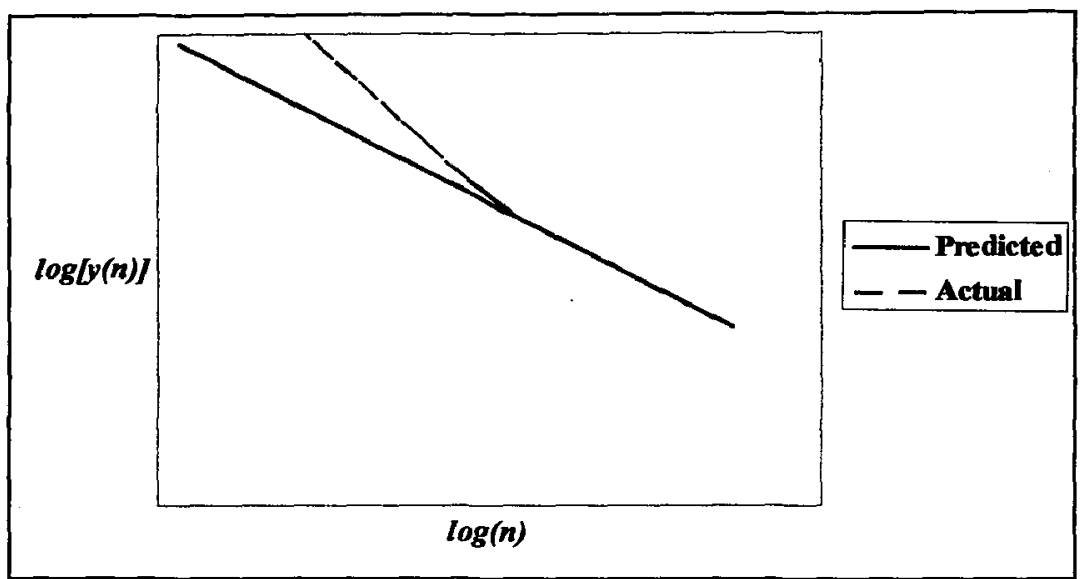

Figure 2.2: Prediction based on late data (Dar-El, 2000)

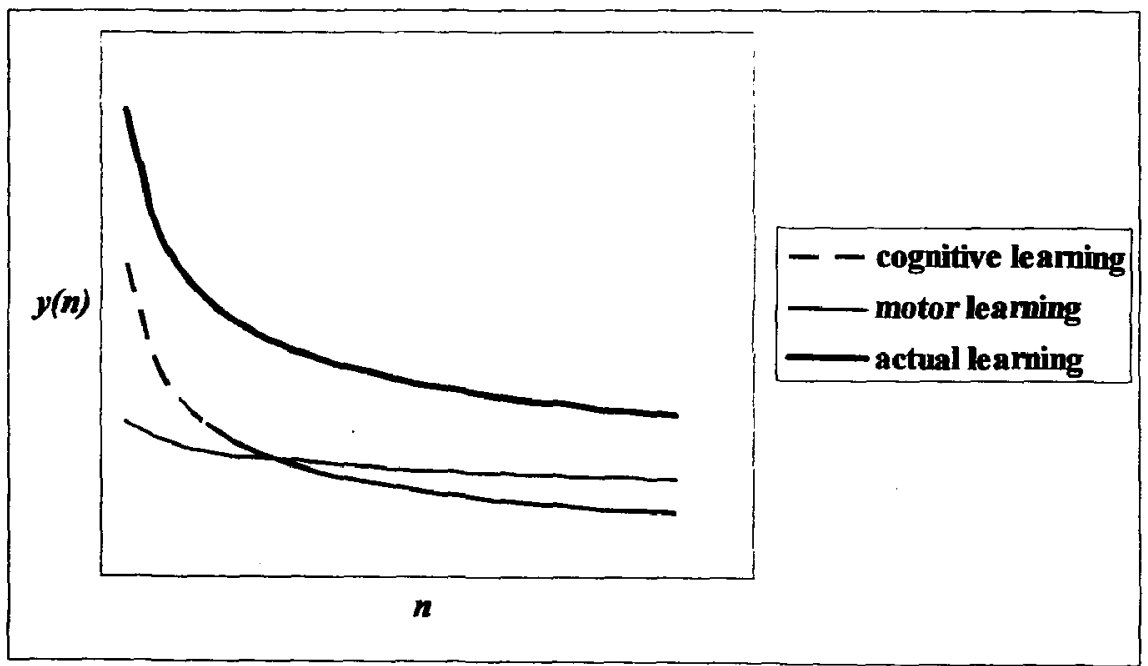

Figure 2.3: The combined effect on learning performance (Dar-El et al., 1995) 
The cognitive portion of the task is designated a learning constant under pure cognitive conditions, $b_{c}$; and the motor portion of the task is designated a learning constant under pure motor conditions, $b_{m}$ (Dar-El et al., 1995). These slopes, and their respective initial processing times, are combined to form the actual dual-learning of the DPLM, it is given by (Dar-El et al., 1995):

$$
y(n)=\left\{y_{c}(1)+y_{m}(1)\right\} \times n^{-b_{*}}
$$

where $y(n)$ is the time to process the $n^{\text {th }}$ unit, $y_{c}(1)$ is the time to process the first unit under purely cognitive conditions, $y_{m}(1)$ is the time to process the first unit under purely motor conditions, and $b$, is the combined learning slope (Dar-El et al., 1995).

\subsubsection{The learn-forget curve model (LFCM) ${ }^{\$}$}

Jaber and Bonney (1996) developed the learn-forget curve model (LFCM) in order to reflect three characteristics of learning highlighted in learning-forgetting literature (Jaber et al., 2003): (1) forgetting is a function of the amount of experience gained prior to an interruption; (2) forgetting is a function of the break length and the learning rate; and, (3) the model should satisfy the two hypotheses that when total forgetting occurs, the processing time reverts to the time to produce the first unit with no prior experience, and the processing time on the learning curve equals the forgetting curve at the point of interruption (Jaber and Bonney, 1996). The LFCM achieved these three criteria respectively by expressing the forgetting slope as a function of the amount of experience prior to the point of interruption, denoted by $u_{i}+n_{i}$, where $u_{i}$ is the amount of experience retained at the beginning of cycle $i$, and $n_{i}$ is the number of units processed in the current cycle $i$ before the interruption; and, the number of units that could have been processed in cycle $i$ if there was no interruption (Jaber and Bonney, 1996):

$$
\phi_{i}=\left[\frac{1-b}{\hat{y}(1)}\left(t\left(u_{i}+n_{i}\right)+d_{i}\right)\right]^{\frac{1}{1-b}}
$$

where $t\left(u_{i}+n_{i}\right)=[y(1) /(1-b)] \times\left(u_{i}+n_{i}\right)^{1-b}$ is the time required to process $u_{i}+n_{i}$ units 
and $d_{i}$ is the length of the interruption interval and $b$ is the learning constant, and the intercept of the forgetting equation in cycle $i$ is given by:

$$
\hat{y}_{i}(1)=y(1)\left(u_{i}+n_{i}\right)^{-\left(b+f_{i}\right)} \text {. }
$$

therefore, the forgetting slope is calculated by:

$$
f_{i}=\frac{b(1-b) \log \left(u_{i}+n_{i}\right)}{\log \left(\alpha_{i}+1\right)}
$$

where $\alpha_{i}$ is found by the following:

$$
\alpha_{i}=D\left[\frac{y(1)\left(u_{i}+n_{i}\right)^{1-b}}{1-b}\right]^{-1},
$$

and $D$ is the time to total forgetting. The time to process the first unit in cycle $i$ is given by:

$$
\tilde{y}_{i}(1)=y(1)\left(u_{i}+1\right)^{-b},
$$

where $u_{i}$, the amount of experience retained from the previous cycle, is found by:

$$
u_{i}=\left(u_{i-1}+n_{i-1}\right)^{\left(b+f_{i}\right) / b}\left(\phi_{i}\right)^{-f_{i} / b}
$$

Using the expressions above, interested readers can confirm that the processing time reverts back to the time to produce the first unit when the interruption interval reaches $D$, and the processing time on the learning curve equals the forgetting curve at the point of interruption (Dar-El et al., 1995). 


\subsection{The dual-phase learning-forgetting model (DPLFM)}

The unique strengths of both the above models, namely, the dual cognitive-motor learning of the DPLM, and the mathematical modeling of the forgetting phenomena of the LFCM were combined by Jaber and Kher (2002) to form the dual-phase learning-forgetting model (DPLFM). Therefore, the DPLFM model has similar relationships to the LFCM with the exception that there is unique learning and forgetting slopes, intersects, levels of transferred experience, and, time to total forgetting parameters for both the cognitive and motor elements of a task (Jaber and Kher, 2002). These dual parameters were then combined to form a processing time for the entire task (both motor and cognitive portions), effective number of units remembered, and, combined learning slopes for every subsequent cycle (Jaber and Kher, 2002). The following is a numerical example that illustrates the calculations involved in the DPLFM. In addition, a table of results is given to examine the behaviour of the DPLFM parameters over several production cycles.

\subsubsection{Numerical example of the DPLFM}

Jaber and Kher (2002) provided a numerical example to illustrate the use of the DPLFM. Reasonable initial estimates of the parameters are given using historical estimates of appropriate cognitive and motor learning rates and $R$ values, where $R$ is the ratio of time for the first unit under purely cognitive conditions to the time for the first unit under purely motor conditions (Jaber and Kher, 2002). In Jaber and Kher (2002), a sensitivity analysis was conducted by varying the length of the interruption interval $\left(d_{i}\right), R$ values, $D_{m}$, and $D_{c}$ values; where $D_{m}$

is the time to totally forget motor elements, and $D_{c}$ is the time to totally forget cognitive elements. In this thesis, however, it was sufficient to just examine the behaviour of the parameters across several cycles by keeping the parameters constant throughout the analysis. For a detailed sensitivity analysis, it is recommended that readers see Jaber and Kher (2002).

Initially, the estimated parameters used for the numerical example are shown in Table 2.1; the settings of these parameters differ only slightly from the numerical example in Jaber and Kher (2002). This numerical example only illustrates the calculations involved to reach the desired parameters for the start of the second cycle. After the sample calculations are shown, a table of parameter values is given in Table 2.2 to display results for five full cycles. 
Table 2.1: Parameter estimations for the DPLFM numerical example

\begin{tabular}{|c|c|c|}
\hline Parameter & Definition & Setting \\
\hline$D_{c}$ & $\begin{array}{l}\text { Time to totally forget } \\
\text { cognitive element of task }\end{array}$ & 45 days \\
\hline$D_{m}$ & $\begin{array}{l}\text { Time to totally forget motor } \\
\text { element of task }\end{array}$ & 250 days \\
\hline$b_{c}$ & Cognitive learning slope & 0.4150 ( $75 \%$ learning rate $)$ \\
\hline$b_{m}$ & Motor learning slope & $0.0740(95 \%$ learning rate $)$ \\
\hline$R$ & $\begin{array}{l}\text { Initial unit processing time: } \\
\text { cognitive-to-motor ratio }\end{array}$ & $1\left[y_{\mathrm{c}}(1) / y_{\mathrm{m}}(1)\right]$ \\
\hline$y(1)$ & Initial unit processing time & 0.25 days \\
\hline$n_{i}$ & $\begin{array}{l}\text { Number of units processed in } \\
\text { each cycle } i \text { (equal for every } \\
\text { cycle) }\end{array}$ & 30 \\
\hline$d_{i}$ & $\begin{array}{l}\text { Interruption interval length } \\
\text { between cycle } i \text { (equal for } \\
\text { every cycle) }\end{array}$ & 15 days \\
\hline
\end{tabular}


Initially, the parameters $\alpha_{i}^{c}$ and $\alpha_{i}{ }^{m}$ are calculated for the first cycle, namely, $\alpha_{1}^{c}$ and $\alpha_{1}^{m}$. These parameters represent the ratio of total forgetting to the time needed to acquire $\left(u_{i}^{c}+n_{i}\right)$ units of cognitive and $\left(u_{i}^{m}+n_{i}\right)$ units of motor experience in the first cycle respectively. This is given by:

$\alpha_{1}^{c}=D_{c}\left[\frac{y_{c}(1)\left(u_{1}{ }^{c}+n_{1}\right)^{1-b_{c}}}{1-b_{c}}\right]^{-1}=45\left[\frac{0.125(0+30)^{1-0.4150}}{1-0.4150}\right]=28.80$

and

$$
\alpha_{1}^{m}=D_{m}\left[\frac{y_{m}(1)\left(u_{1}^{m}+n_{1}\right)^{1-b_{m}}}{1-b_{m}}\right]^{-1}=250\left[\frac{0.125(0+30)^{1-0.0740}}{1-0.0740}\right]=79.40
$$

The DPLFM assigns learning slopes for the cognitive and motor portions of a task, given as $b_{c}$ and $b_{m}$ respectively. The combined slope $b_{1 *}$ for the first cycle is given by:

$$
b_{1 *}(n)=b_{c}-\frac{\log \left[\left(R+\left(u_{1}+n\right)^{b_{c}-b_{m}}\right) /(R+1)\right]}{\log \left(u_{1}+n\right)},
$$

substituting the values gives:

$$
b_{1 *}(30)=0.4150-\frac{\log \left[\left(1+(0+30)^{0.4150-0.0740}\right) /(1+1)\right]}{\log (0+30)}=0.198 \text {. }
$$

The forgetting slopes for the first cycle under pure cognitive and motor conditions are given respectively by:

$$
f_{1}^{c}=\frac{b_{c}\left(1-b_{c}\right) \log \left(u_{1}^{c}+n\right)}{\log \left(1+\alpha_{1}^{c}\right)}=\frac{0.4150(1-0.4150) \log (0+30)}{\log (1+28.80)}=0.243,
$$

and

$$
f_{1}^{m}=\frac{b_{m}\left(1-b_{m}\right) \log \left(u_{1}^{m}+n\right)}{\log \left(1+\alpha_{1}^{m}\right)}=\frac{0.0740(1-0.0740) \log (0+30)}{\log (1+79.40)}=0.053
$$


The time needed to acquire $\left(u_{i}^{c}+n_{i}\right)$ units of cognitive experience and $\left(u_{i}^{m}+n_{i}\right)$ units of motor experience in the first cycle is given by:

$$
y\left(u_{1}^{c}+n_{1}\right)=\frac{y_{c}(1)\left(u_{1}^{c}+n\right)^{1-b_{c}}}{1-b_{c}}=\frac{0.125(0+30)^{1-0.4150}}{1-0.4150}=1.563,
$$

and

$$
y\left(u_{1}^{m}+n_{1}\right)=\frac{y_{m}(1)\left(u_{1}^{m}+n\right)^{1-b_{m}}}{1-b_{m}}=\frac{0.125(0+30)^{1-0.0740}}{1-0.0740}=3.149
$$

Therefore, the time to acquire $\left(u_{1}+n_{1}\right)$ units of combined experience in the first cycle is given by:

$$
y\left(u_{1}+n_{1}\right)=\frac{y(1)\left(u_{1}+n\right)^{1-b_{1 *}}}{1-b_{1^{*}}}=\frac{0.25(0+30)^{1-0.198}}{1-0.198}=4.769
$$

The combined forgetting slope $f^{*}$ for the first cycle is given by:

$$
f_{1}^{*}=\frac{b_{1^{*}}\left(1-b_{1^{*}}\right) \log \left(u_{1}+n_{1}\right)}{\log \left[1+\frac{\max \left\{D_{c}, D_{m}\right\}}{y\left(u_{1}+n_{1}\right)}\right]}=\frac{0.198(1-0.198) \log (0+30)}{\log [1+250 / 4.769]}=0.136
$$

The forget curve intercept for the first cycle is given by:

$$
\hat{y}_{1}(1)=y(1)\left(u_{1}+n_{1}\right)^{-\left(b_{1}+f_{1^{*}}\right)}=0.25(0+30)^{-(0.198+0.136)}=0.080
$$

If there was not an interruption in the production process, and if learning continued throughout this process, a theoretical number of units could have been produced. The number of both cognitive units $\left(\phi_{1}^{c}\right)$ and motor units $\left(\phi_{1}^{m}\right)$ that could have been processed in the first cycle if there was not an interruption is given as follows:

$$
\begin{aligned}
& \phi_{1}{ }^{c}=\left[\frac{1-b_{c}}{y_{c}(1)}\left[y\left(u_{1}{ }^{c}+n_{1}\right)+d_{1}\right]\right]^{1 / 1-b_{c}} \\
& =\left[\frac{1-0.4150}{0.125}(1.563+15)\right]^{1 / 1-0.4150}=1698,
\end{aligned}
$$


and

$$
\begin{aligned}
& \phi_{1}^{m}=\left[\frac{1-b_{m}}{y_{m}(1)}\left[y\left(u_{1}^{m}+n_{1}\right)+d_{1}\right]\right]^{1 / 1-b_{m}} \\
& =\left[\frac{1-0.0740}{0.125}(3.149+15)\right]^{1 / 1-0.0740}=199 .
\end{aligned}
$$

Since the DPLFM models a partial transmission of learning after each interruption, this retained experience must be accounted for in successive cycles. The DPLFM does this by calculating the equivalent number of units of experience retained (or transferred) to the subsequent cycle. Therefore, the number of cognitive and motor units of experience retained at the start of the second cycle is given by:

$$
\begin{aligned}
u_{2}^{c}=\left(u_{1}^{c}+\right. & \left.n_{1}\right)^{\left(b_{c}+f_{1}^{c}\right) / b_{c}} \times\left(\phi_{1}^{c}\right)^{-f_{1}^{c} / b_{c}} \\
& =(0+30)^{(0.4150+0.243) / 0.4150} \times(1698)^{-0.243 / 0.4150}=3^{,}
\end{aligned}
$$

and

$$
\begin{aligned}
u_{2}^{m}=\left(u_{1}^{m}+n_{1}\right)^{\left(b_{m}+f_{1}^{m}\right) / b_{m}} \times\left(\phi_{1}^{m}\right)^{-f_{1}^{m} / b_{m}} \\
=(0+30)^{(0.0740+0.053) / 0.0740} \times(199)^{-0.053 / 0.0740}=8
\end{aligned}
$$

The processing time for the first unit in the second cycle is now found as follows:

$$
\begin{aligned}
y_{2}(1)= & y_{2}^{c}(1)+y_{2}^{m}(1)=y_{c}(1)\left(u_{2}^{c}+1\right)^{-b_{c}}+y_{m}(1)\left(u_{2}{ }^{m}+1\right)^{-b_{m}} \\
& =0.125(3+1)^{-0.4150}+0.125(8+1)^{-0.0740} \\
& =0.177 \text { days. }
\end{aligned}
$$

The value of 0.177 follows the logic of the assumption that experience is still transferred to the next cycle causing $y_{2}(1)<y_{1}(1)$ as long as $d_{i}<D$. The number of units of experience remembered at the start of the second cycle is given by:

$u_{2}=\left[\frac{y_{2}(1)}{y_{1}(1)}\right]^{-1 / b_{1}(30)}-1=\left[\frac{0.177}{0.250}\right]^{-1 / 0.198}-1=5$ 
Therefore, since the processing time and the number of transferred units of experience for the first unit of the second cycle is now known for both cognitive and motor elements, all of the previous calculations can be performed for the second cycle and all other successive production cycles. For interest, the combined learning slope for the second cycle is given by:

$b_{2^{*}}(n)=b_{c}-\frac{\log \left[\left(R+\left(u_{2}+n\right)^{b_{c}-b_{m}}\right) /(R+1)\right]}{\log \left(u_{2}+n\right)}$,

substituting the values give:

$b_{2 *}(30)=0.4150-\frac{\log \left[\left(1+(5+30)^{0.4150-0.0740}\right) /(1+1)\right]}{\log (5+30)}=0.196$

Likewise, the parameters for the first five production cycles are calculated and summarized in Table 2.2. As shown in Table 2.2, the behaviour of the initial unit processing time of each cycle decreases at a non-uniform rate through successive cycles. This implies that some experience is transferred after the interruption period between cycles. The equivalent units of experience transferred between cycles, or $u_{i}$, represents the degree by which forgetting has caused the experience of the worker to rise back up the learning curve. The increasing level of $u_{i}$ suggests that this corresponds to more transferred experience between cycles as the number of cycles increase. However, it appears that $u_{i}$ slowly asymptotes to some fixed value after successive cycles. Also, the combined cognitive/motor learning slope $b_{i^{*}}$ appears to behave in the same manner, suggesting that, in this particular example, the rate at which the worker learns gradually decreases with successive cycles.

In summary, the DPLFM is the only learning and forgetting model that closely models seven characteristics of learning as summarized by Jaber et al. (2003), and it theoretically models cognitive and motor learning elements that were validated experimentally. 
Table 2.2: Initial five production cycle parameters of the DPLFM numerical example

\begin{tabular}{|l|l|l|l|l|}
\hline Cycle $i$ & $n$ & $y_{i}(1)$ & $u_{i}$ & $b_{i^{*}}$ \\
\hline 1 & 30 & 0.250 & 0 & 0.198 \\
\hline 2 & 30 & 0.177 & 5 & 0.196 \\
\hline 3 & 30 & 0.175 & 6 & 0.195 \\
\hline 4 & 30 & 0.174 & 6 & 0.195 \\
\hline 5 & 30 & 0.174 & 6 & 0.195 \\
\hline
\end{tabular}

\subsection{Chapter 2 summary}

The previous chapter 2 has summarized the development of the dual-phase learning-forgetting model (DPLFM) and has illustrated its behaviour with a numerical example. The following chapter 3 summarizes the modeling or experimental designs used in the three models of this thesis. 


\section{Chapter 3: experimental design and modeling}

\subsection{Experimental design of model $A$}

There are two main components to any experimental problem: the design of the experiment and the statistical analysis of the results. This chapter outlines the experimental design for the models in this thesis. The statistical analysis of the results is not necessary or possible since the experiment is deterministic. That is, all of the models in this thesis use a series of functions and logical statements, and each observation for each cell of the experiment is identical to the next. Consequently, no statistical methods are necessary since the results are definite and not probabilistic.

Montgomery (1997) stated three basic principles of experimental design: (1) replication, (2) randomization, and, (3) blocking. The experiments in model $\mathrm{A}$, model $\mathrm{B}$, and model $\mathrm{C}$ do not have any errors due to variability because of their deterministic input parameters, and therefore no replications or randomizations of replications are necessary. Also, blocking is not necessary because nuisance sources of variability do not exist in a deterministic model. However, this thesis does conduct a mathematical test in which changes are made to the input variables that result in changes to output responses, and hence, this thesis does use experiments in all of its models. As a result, this thesis employs an experimental design for all of its models. An effective experiment includes the following seven steps (Montgomery, 1997): (1) recognition and statement of the problem, (2) choice of factors, levels, and ranges, (3) selection of the response variable, (4) choice of experimental design, (5) performing the experiment, (6) statistical analysis of the data, and, (7) conclusions and recommendations. However, since the models in this thesis are deterministic, the statistical analysis of the data is excluded. Instead, all the main factor effects and factor interactions are examined in a logical and qualitative fashion. 


\subsubsection{Recognition and statement of the problem}

The purpose of Jaber et al. (2003) was to see the relative effect of modeling a DRC shop system with worker learning and forgetting following the LFCM of Jaber and Bonney (1996) versus the VRVF learning model of Carlson and Rowe (1976). Four other experimental factors were varied in addition to the type of learning model.

The problem or purpose of model $\mathrm{A}$ is to determine the effect of the task-type factor on DRC shop performance using the DPLFM instead of the VRVF or the LFCM (used in Jaber et al. (2003)) to model worker learning and forgetting. Namely, the learning rate of the worker is the new variable in the DRC system and it varies in relation to the type of task being processed. The degree and pattern of the difference in results obtained by including the task-type factor (variable learning rate) in model A versus the results of a fixed task-type (learning rate) in Jaber et al. (2003) is commented upon and conclusions are drawn for the reasons for the change in output as it relates to a DRC system context.

\subsubsection{Choice of factors, levels, and ranges}

Jaber et al. (2003) used a five factor experiment with different levels within each factor. The experimental factors and their corresponding levels are summarized in Table 3.1. Montgomery (1997) stated that relevant process knowledge in the form of practical experience or theoretical understanding is necessary in order to set appropriate factor levels and ranges of those levels. The levels chosen in Jaber et al. (2003) have been justified empirically by referencing studies in DRC research. For example, as shown in Table 3.1, the levels of worker flexibility chosen were justified empirically by a study by Malhotra et al. (1993). The levels chosen for worker forgetting rates were also justified by Malhotra et al. (1993) as representing a relatively challenging machine-paced environment. The range of the centralization of control levels is appropriate given the transfer time penalties associated with the levels of forgetting used (Kher et al., 1999). The range of the upfront training levels were chosen to reflect the trade off between the choice to take advantage of flexibility as soon as possible $(F A P-0)$ and the desire for greater worker efficiency in the long run (Kher et al., 1999). However, even though training periods of zero $(F A P-0)$, one $(F A P-1)$, and two $(F A P-2)$ times the standard times seem appropriate, no empirical evidence was given for choosing these levels (Kher et al., 1999). Finally, the two levels for learning and forgetting were justified in the original development of these models by 
Table 3.1: Experimental factors used in the study of Jaber et al. (2003)

\begin{tabular}{|c|c|c|}
\hline Experimental factor & Levels per factor & Notes \\
\hline $\begin{array}{l}\text { Level of worker flexibility } \\
(N)\end{array}$ & 2 & $\begin{array}{l}\text { The worker is either trained for two } \\
\text { or three tasks/stages. Note that a } \\
\text { maximum of three workstations is } \\
\text { used because of the Malhotra et al. } \\
\text { (1993) finding that cross-training } \\
\text { workers in more than three } \\
\text { departments worsens system } \\
\text { performance. }\end{array}$ \\
\hline $\begin{array}{l}\text { Centralization of control } \\
(C)\end{array}$ & 3 & $\begin{array}{l}\text { The worker moves from the station } \\
\text { after a fixed number of jobs are } \\
\text { completed, i.e.: after batch sizes of } \\
10,25 \text {, and } 250 . \text { Kher et al. ( } 1999) \\
\text { suggested that a batch size transfer } \\
\text { less than } 10 \text { or greater than } 250 \\
\text { causes too many transfer time } \\
\text { penalties to be effective. }\end{array}$ \\
\hline $\begin{array}{l}\text { Worker forgetting rate } \\
(F R)\end{array}$ & 3 & $\begin{array}{l}\text { Three different worker forgetting rate } \\
\text { levels of } 85 \%, 80 \% \text {, and } 95 \% \text {. The } \\
\text { range of these levels is the same as in } \\
\text { Jaber et al. (2003). Kher et al. (1999) } \\
\text { used the same range and commented } \\
\text { that it represented reasonably } \\
\text { challenging yet sufficiently broad } \\
\text { forgetting conditions. }\end{array}$ \\
\hline $\begin{array}{l}\text { Extent of upfront worker } \\
\text { training }(F A P)\end{array}$ & 3 & $\begin{array}{l}F A P-0, F A P-1, \text { and } \\
F A P-2 \text { which corresponds to: no } \\
\text { initial training, the worker must } \\
\text { process until standard time is reached } \\
\left(370 \text { units }{ }^{* *}\right) \text { in the first cycle, and, } \\
\text { the worker must process twice this } \\
\text { amount }(740) \text { in the first cycle } \\
\text { respectively before any transfers } \\
\text { occur. }\end{array}$ \\
\hline $\begin{array}{l}\text { Worker learning-forgetting } \\
\text { model }\end{array}$ & 2 & $\begin{array}{l}\text { Worker learning-forgetting is } \\
\text { modeled according to the VRVF and } \\
\text { the LFCM. }\end{array}$ \\
\hline
\end{tabular}

. Based on equation (1) in Kher et al. (1999) for an $85 \%$ learning environment 
Carlson and Rowe (1976) (VRVF) and Jaber and Bonney (1996) (LFCM). The comprehensive summary of assumptions and results of Jaber et al. (2003), and the assumptions and results of the models of this thesis as they relate to DRC system research are given in the DRC summary matrix in the appendix, see section 7.1. The experimental factors of Jaber et al. (2003) and all the models in this thesis are also included in this DRC summary matrix ${ }^{\dagger \dagger}$. In model $\mathrm{A}$, the first four experimental factors of Jaber et al. (2003) are used, as described in Table 3.1 (excluding the learning-forgetting model factor). The additional experimental factors used in this thesis are the initial unit processing time cognitive-to-motor ratio $(R)$, and the introduction of four different task-types with respect to both cognitive and motor learning elements of the task. The additional experimental factors used in model A are summarized in Table 3.2. As a result, from Tables 3.1 and 3.2 , this experiment is a 2 (level of worker flexibility) $\times 3$ (centralization of control ('when' rule) $) \times 3$ (worker forgetting rate) $\times 3$ (extent of upfront worker training) $\times 4$ (task-type) $\times 3(R)$ model yielding 648 individual data points, where each data point represents the performance measure of the average processing (service) time ( $A P T$ ) calculated over approximately 3000 jobs per station, the same performance measure used by Jaber et al. (2003). In Jaber et al. (2003), a fixed learning rate was assumed to apply to the task as a whole. However, in model A, the composition of the learning rate of each task is specified using unique cognitive and motor learning rates. Four different task-types, a predominantly cognitive task $\left(T_{C}\right)$, a motor task $\left(T_{M}\right)$, a median task $\left(T_{M e d}\right)$, and a uniform task $\left(T_{U}\right)$ are defined by their own respective cognitive and motor learning rates. In model A, as in Jaber et al. (2003), there is no difference in factors between stations. The levels and ranges for the learning rates of the four task-types are supported by an initial parameter learning estimation section by Dar-El et al. (1995). The classification of learning rates as a function of task-type is given in Figure 3.1. Dar-El et al. (1995) classified high cognitive tasks (C1) $L R=70 \%$ to $75 \%$, more cognitive than motor tasks (C2) $L R=75 \%$ to $80 \%$, more motor than cognitive tasks (M2) $L R=80 \%$ to $85 \%$, and high motor tasks (M1) $L R=80 \%$ to $85 \%$. In model A, the cognitive task $T_{C}$, has a cognitive learning rate equal to the mean of the 'high cognitive task' range, or $72.5 \%$, and a motor learning rate equal to the mean of the 'more motor than cognitive' task range, or $82.5 \%$.

\footnotetext{
t† Please note that the matrix clearly distinguishes the similarities and differences between models and highlights the assumptions of each model in bold.
} 
Table 3.2: Additional experimental factors used in model $A$

\begin{tabular}{|l|l|l|}
\hline Experimental factor & Levels per factor & Notes \\
\hline Task-type & 4 & $\begin{array}{l}\text { A predominantly cognitive task } T_{C}, \text { and } \\
\text { motor task } T_{M}, \text { are distinguished from } \\
\text { each other with their own composite } \\
\text { learning rates. The respective cognitive } \\
\text { and motor learning rates of cognitive task } \\
T_{C} \text { are as follows: } \\
L R_{C}=72.5 \%, L R_{M}=82.5 \% \text {; and for } \\
\text { motor task } T_{M}: L R_{C}=77.5 \%, L R_{M} \\
=87.5 \% \text {. The median task } T_{M e d} \text { and } \\
\text { uniform task } T_{U} \text { have cognitive and } \\
\text { motor learning rates of } L R_{C}=75 \%, \\
L R_{M}=85 \%, \text { and } L R_{C}=L R_{M}= \\
80 \% \text { respectively. }\end{array}$ \\
\hline$R$ & & $\begin{array}{l}\text { Initial unit processing time: cognitive-to- } \\
\left.\text { motor ratio [ } y_{c}(1) / y_{m}(1)\right] \text {. The three } \\
\text { levels are set at } 1 / 3,1, \text { and 3. These ratios } \\
\text { correspond to those empirically observed } \\
\text { by Dar El et al. (1995) and used in a } \\
\text { numerical example by Jaber and Kher } \\
(2002) .\end{array}$ \\
\hline &
\end{tabular}

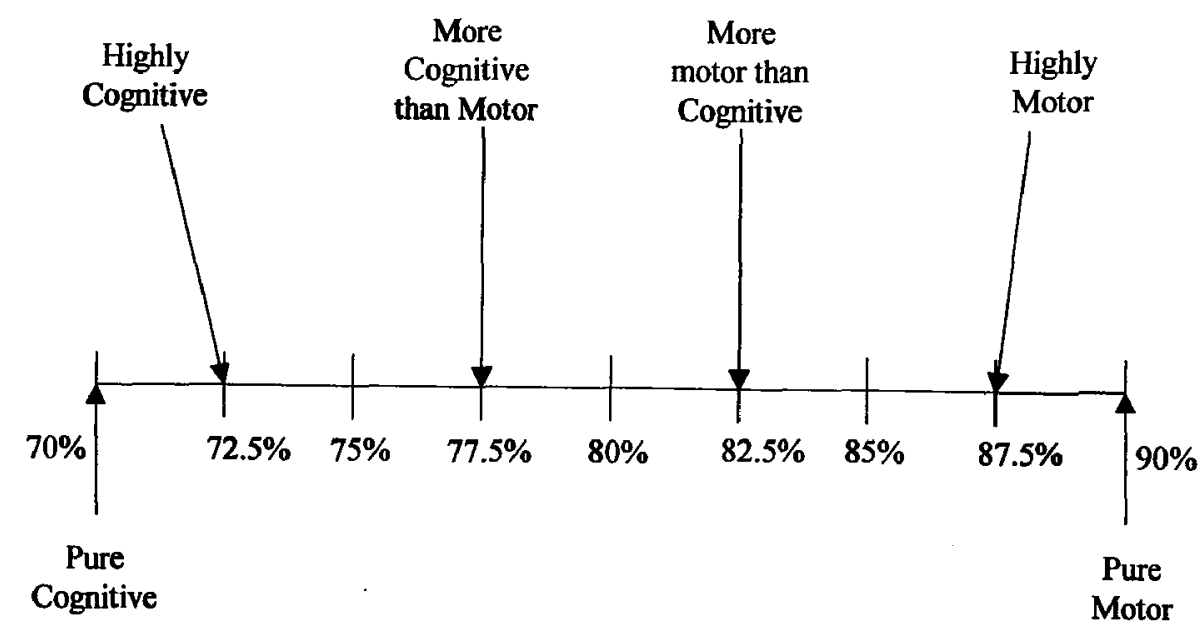

Figure 3.1: Classification of learning slopes (Dar-El et al, 1995) 
The motor task $T_{M}$ has a cognitive learning rate equal to the mean of the 'more cognitive than motor' task range, or $77.5 \%$, and a motor learning rate equal to the mean of the 'high motor task' range, or $87.5 \%$. The median task $T_{M e d}$ represents a task that is neither predominantly cognitive nor motor, and hence, has a cognitive and motor learning range spread equally across the median of the learning range in Figure 4.5 with a cognitive learning rate equal to $75 \%$, and a motor learning rate equal to $85 \%$. The uniform task $T_{U}$ represents a task that has an average learning rate equal to that of the median task $T_{\text {Med }}(80 \%)$; however, this task has no distinctive cognitive and motor elements and therefore has both cognitive and motor learning rates equal to 80\%. The values and ranges of the four task-types are shown in Figure 3.2. Model A also uses two additional parameters. These parameters, their values, and the justification for their levels are listed in Table 3.3. The parameter $D_{c}$ in each station is given by substituting equation (2.8) into (2.12) to get:

$$
f_{1}^{c}=\frac{b_{c}\left(1-b_{c}\right) \log \left(u_{1}^{c}+n\right)}{\log \left(1+\frac{D_{c}\left(1-b_{c}\right)}{y_{c}(1)\left(u_{1}{ }^{c}+n_{1}\right)^{1-b_{c}}}\right)},
$$

and solving for $D_{c}$ gives:

$$
D_{c}=\left(10^{\frac{b_{c}\left(1-b_{c}\right) \log \left(u_{1}^{c}+n\right)}{f_{1}^{c}}}-1\right) \times\left(\frac{y_{c}(1)\left(u_{1}^{c}+n_{1}\right)^{1-b_{c}}}{1-b_{c}}\right)
$$

$D_{m}$ is found similarly and is given by:

$$
D_{m}=\left(10^{\frac{b_{m}\left(1-b_{m}\right) \log \left(u_{1}^{m}+n\right)}{f_{1}^{m}}}-1\right) \times\left(\frac{y_{m}(1)\left(u_{1}^{m}+n_{1}\right)^{1-b_{m}}}{1-b_{m}}\right)
$$

where $f_{1}^{c}, f_{1}^{m}, n_{1}$, are the specified initial cognitive and motor forgetting slopes and the 


\section{Motor Tass( (Im)}
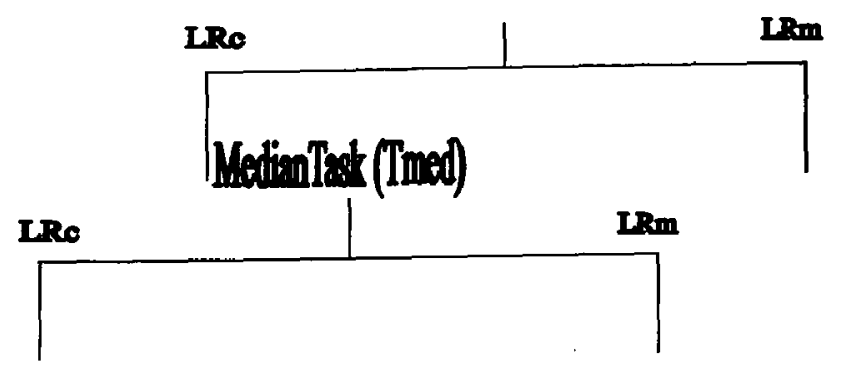

20\% $725 \% \quad 75 \% \quad 775 \% \quad 80 \% \quad 825 \% \quad 85 \% \quad 875 \% \quad 90 \%$

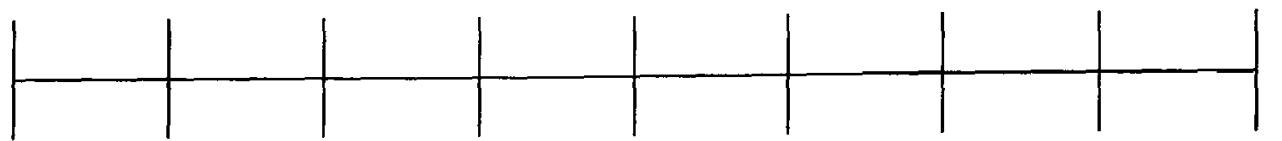

Pare Cognitive

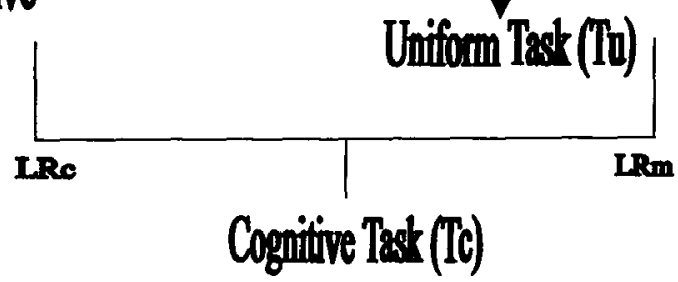

Pure Motor

Figure 3.2: The ranges of learning rates for the four task-types in model A

Table 3.3: Additional parameters related to time-for-total-forgetting used in model $A$.

\begin{tabular}{|l|l|l|l|}
\hline Parameter & Definition & Level & \begin{tabular}{l} 
Justification \\
\hline$D_{c}$
\end{tabular} \\
$\begin{array}{l}\text { Time to totally } \\
\text { forget cognitive } \\
\text { elements of a task }\end{array}$ & $\begin{array}{l}\text { The time to total forgetting }(D) \text { is } \\
\text { a function of the forgetting rate, } \\
\text { learning slope, number of units } \\
\text { produced and the time to produce } \\
\text { these units. However, in the } \\
\text { DPLFM, there is a unique time to } \\
\text { forgetting parameter for both } \\
\text { cognitive and motor elements. }\end{array}$ & $\begin{array}{l}\text { Faster learned tasks are also } \\
\text { forgotten faster, given that } \\
\text { cognitive tasks are learned a } \\
\text { a faster rate (Jaber et al., } \\
\text { 2003). These levels of time } \\
\text { to total forgetting were also } \\
\text { used in Jaber and Kher } \\
(2002) .\end{array}$ \\
\hline$D_{m}$ & $\begin{array}{l}\text { Time to totally } \\
\text { forget motor } \\
\text { elements of a task }\end{array}$ & $\begin{array}{l}\text { The time to total forgetting }(D) \text { is } \\
\text { a function of the forgetting rate, } \\
\text { learning slope, number of units } \\
\text { produced and the time to produce } \\
\text { these units. However, in the } \\
\text { DPLFM, there is a unique time to } \\
\text { forgetting parameter for both } \\
\text { cognitive and motor elements. }\end{array}$ & Same as above. \\
\hline
\end{tabular}


number of units processed in the first cycle respectively. Also, in model A, the determination of the number of units needed to reach the standard time of the worker $\left(\boldsymbol{n}_{s}\right)$ necessitated solving for $n_{s}$ in the following expression using a simple bisection numerical technique:

$$
y_{c}(1) n_{s}^{-b_{c}}+y_{m}(1) n_{s}^{-b_{m}}=y_{s} \text {. }
$$

\subsubsection{Selection of the response variables}

The response variable in model $\mathrm{A}$ is the performance measure of the DRC system in question, namely: average processing (service) time ( $A P T$ ) calculated over approximately 3000 jobs per station (APT). This response variable is consistent with the one used in Jaber et al. (2003).

The results of model A are compared to those of Jaber et al. (2003). Also, the results are interpreted as they pertain to the five possible issues that affect DRC shop performance measures: worker flexibility, centralization of control, worker assignment, queue disciplines, and cost of transferring workers (Hottenstein and Bowman, 1998). This comparison is conducted for every model in this thesis. These issues are summarized in the results/conclusions section of the summary matrix in the appendix (see section 7.1.2) and are included for the results of every model examined in this thesis.

\subsubsection{Choice of experimental design}

As previously discussed, model $\mathrm{A}$ uses deterministic input parameters, and hence the results do not require the statistical tools of replication, randomization, or blocking. However, a judgement had to be made to determine whether the difference in outputs obtained by using the DPLFM instead of the VRVF and the LFCM learning models is of any practical significance. The implications of the results of model $A$ are also discussed as they pertain to five possible DRC shop performance issues discussed by Hottenstein and Bowman (1998).

\subsubsection{Modeling methodology}

The calculations for model A were performed using an Excel spreadsheet. Any necessary programming was performed in Excel-Visual Basic. 


\subsection{Experimental design of model B}

Task similarity can be defined as the ratio of shared task attributes (between tasks) and the total task attributes of all tasks, where each task attribute is inherent to the task (i.e. irrespective to who works on the task), is identical in each task, and does not depend on other attributes. This simplification of the definition of task similarity and task attributes is given for ease of quantification.

Intuitively, performing a similar task during a work interruption yields less forgetting losses than performing a task that is totally dissimilar. Jaber et al. (2003) used this reasoning to remodel the forgetting losses associated with the LFCM by assuming that tasks have various levels of similarity. This was done by assigning a similarity coefficient that measures the degree of commonality between task attributes for two and three tasks. For example, a similarity coefficient of $s=0$ and $s=1$ represent tasks that are totally dissimilar and totally similar respectively. They then remodelled the LFCM and determined how the task similarity factor affected the previous assumption of no task similarities. The similarity coefficient for two tasks is calculated as follows (Jaber et al., 2003):

$$
s=\frac{2[N\{T(1) \cap T(2)\}]}{N\{T(1)\}+N\{T(2)\}}
$$

where $N\{X\}$ in the denominator is the number of attributes in task $X$. The numerator represents the number of common attributes between tasks multiplied by two. The similarity coefficients for three tasks consist of one three-way coefficient and three two-way coefficients.

They are calculated as follows (Jaber et al., 2003):

the three-way coefficient is given by,

$$
s_{(1,2,3)}=\frac{3[N\{T(1) \cap T(2) \cap T(3)\}]}{N\{T(1)\}+N\{T(2)\}+N\{T(3)\}},
$$


the two-way coefficient is given by,

$$
s_{(1,2)}=\frac{2\left[N\left\{T(1)^{*} \cap T(2)^{*}\right\}\right]}{N\left\{T(1)^{*}\right\}+N\left\{T(2)^{*}\right\}}
$$

where $T(i)^{*}=T(i)-\{T(1) \cap T(2) \cap T(3)\}$ for $i=1,2$. The other two-way coefficients $s_{(2,3)}$ and $s_{(1,3)}$ are calculated similarly. For a detailed numerical example of the determination of similarity coefficients see Jaber et al., (2003). The determination of the forget curve intercept equation (2.18) of the DPLFM is then modified for two similar tasks as follows:

$$
\hat{y}_{1 i}(1)=\left\{\begin{array}{l}
(1-s) y(1)\left(n+u_{i}\right)^{-\left(b_{i *}+f_{i}^{*}\right)}+s y(1)\left(n+u_{i}\right)^{-b_{i^{*}},}, \text { if } n+u_{i}<n_{s}, \\
(1-s) y_{s}\left(n+u_{i}\right)^{-f_{i}^{*}}+s y_{s}, \text { otherwise }
\end{array}\right.
$$

where $y_{s}$ is the standard time and $n_{s}$ is the required effective quantity to reach standard time. Also, the determination of the forget curve intercept equation (2.18) of the DPLFM is then modified for three similar tasks as follows:

$$
\hat{y}_{1 i}(1)=\left\{\begin{array}{l}
\left(1-s_{j k}-s_{j k l}\right) y(1)\left(n+u_{i}\right)^{-\left(b_{i}+f_{i}^{*}\right)}+\left(s_{j k}+s_{j k l}\right) y(1)\left(n+u_{i}\right)^{-b_{i *},} \text { if } n+u_{i}<n_{s}, \\
\left(1-s_{j k}-s_{j k l}\right) y(1)\left(n+u_{i}\right)^{-f_{i}^{*}}+\left(s_{j k}+s_{j k l}\right) y_{s}, \text { otherwise }
\end{array}\right.
$$

where $s_{j k}$ is the similarity coefficient of the task of the current station $j$ and the task at the successor station $k$, and $s_{j k l}$ is the three-way similarity coefficient.

\subsubsection{Recognition and statement of the problem}

Jaber et al. (2003) examined how the inclusion of the task similarity factor affected the results of the original assumption of no task similarity. The purpose of model $B$ of this thesis is to determine how the inclusion of the task similarity factor affects the results of model $A$ and how this compares with Jaber et al. (2003), where the LFCM was used. 


\subsubsection{Choice of factors, levels, and ranges}

In model B, the same experimental factors of model A are used. The additional experimental factor in model $\mathrm{B}$ is the degree of similarity between tasks, this is denoted by $\boldsymbol{s}$. For consistency, the levels in model B are identical to those chosen by Jaber et al. (2003). They are listed for two and three tasks in Table 3.4. As shown in Table 3.4, there are five similarity levels for two tasks and four similarity levels for three tasks. The ranges for the levels for two tasks seem appropriate because the degree of similarity increases in a constant manner in a wide range from two totally dissimilar tasks, $s=0$, to two very similar tasks $s=0.8$. Also, for three tasks, the levels chosen represent an ever-increasing degree of similarity, with the highest level $\left(S_{3}\right)$ having all three tasks sharing many common task attributes. As a result, from Tables 3.1, 3.2 and 3.4, the experiment in model B is a [3(centralization of control ('when' rule)) $\times 3$ (worker forgetting rate) $\times 3$ (extent of upfront worker training) $\times 4$ (task-type) $\times 3(R) \times 5$ (levels of similarity when $N=2)]+[3($ centralization of control ('when' rule) $) \times 3$ (worker forgetting rate) $\times 3$ (extent of upfront worker training) $\times 4$ (task-type) $\times 3(R) \times 4$ (levels of similarity when $N=3$ )] model yielding 2916 individual data points, where each data point represents the performance measure of the average processing (service) time calculated over approximately 3000 jobs per station, the same performance measure used in model A and in Jaber et al. (2003).

\subsubsection{Selection of the response variables}

The response variable in model $B$ is the same as in model A. 
Table 3.4: The levels of the factors in model $B$

\begin{tabular}{|l|l|l|l|l|l|}
\hline$N$ & \multicolumn{4}{|l|}{ Factor levels } \\
\hline $\mathbf{2}$ & $\boldsymbol{s}=0.0$ & $\boldsymbol{s}=0.2$ & $\boldsymbol{s}=0.4$ & $\boldsymbol{s}=0.6$ & $\boldsymbol{s}=\mathbf{0 . 8}$ \\
\hline $\mathbf{3}$ & Level $S_{0}$ & Level $S_{1}$ & Level $S_{2}$ & Level $S_{3}$ \\
& $s_{(I, 2)}=0.0$ & $s_{(I, 2)}=0.2$ & $s_{(I, 2)}=0.25$ & $s_{(I, 2)}=0.0$ \\
& $s_{(2,3)}=0.0$ & $s_{(2,3)}=0.2$ & $s_{(2,3)}=0.25$ & $s_{(2,3)}=0.0$ \\
& $s_{(1,3)}=0.0$ & $s_{(l, 3)}=0.2$ & $s_{(I, 3)}=0.25$ & $s_{(I, 3)}=0.0$ \\
& $s_{(I, 2,3)}=0.0$ & $s_{(I, 2,3)}=0.0$ & $s_{(I, 2,3)}=0.2$ & $s_{(l, 2,3)}=0.8$ \\
\hline
\end{tabular}

Table 3.5: Input and output factors used in the formulation of the multivariate function in model C

\begin{tabular}{|c|c|c|c|}
\hline \multicolumn{2}{|l|}{ Factor } & \multirow{2}{*}{$\begin{array}{l}\text { Levels/ranges } \\
s:\{0.0,0.2,0.4,0.6,0.8\}\end{array}$} & \multirow{2}{*}{$\begin{array}{l}\text { Justification } \\
\text { Same levels and ranges } \\
\text { used in Jaber et al. } \\
(2003) \text {. }\end{array}$} \\
\hline Input factors & $\begin{array}{l}\text { Degree of task } \\
\text { similarity }(s)\end{array}$ & & \\
\hline & $\begin{array}{l}\text { Initial processing time } \\
\text { to standard time ratio } \\
(\rho)\end{array}$ & $\rho:\{2,3,4,5,6\}$ & $\begin{array}{l}\text { Malhotra et al. ( } 1993) \\
\text { used a } \rho \text { value of } 2 \\
\text { and } 4 \text { to represent a low } \\
\text { and high initial cost of } \\
\text { learning respectively. } \\
\text { Model } C \text { also includes } \\
\text { the effect of a more } \\
\text { severe initial learning } \\
\text { loss }(\rho=5,6)\end{array}$ \\
\hline & $\begin{array}{l}\text { Worker learning rate } \\
(L R) \text {, where } \\
L R=100 \times 2^{-b}\end{array}$ & $\begin{array}{l}L R: \\
\{70 \%, 75 \%, 80 \%, 85 \%, 90 \%\}\end{array}$ & $\begin{array}{l}\text { As in model } A, \text { the } \\
\text { range of the worker } \\
\text { learning rate } \\
\text { encompasses the entire } \\
\text { estimated range as } \\
\text { noted by Dar-El et al. } \\
\text { (1995a), see Figure } 3.1 \text {. }\end{array}$ \\
\hline & $\begin{array}{l}\text { Worker forgetting rate } \\
(F R) \text {, where } \\
F R=100 \times 2^{f}\end{array}$ & $\begin{array}{l}F R: \\
\{95 \%, 92.5 \%, 90 \%, 87.5 \%, 85 \%\}\end{array}$ & $\begin{array}{l}\text { Same as in model } A \text {, see } \\
\text { Table } 3.1 \text {. }\end{array}$ \\
\hline \multirow[t]{2}{*}{$\begin{array}{l}\text { Optimal output } \\
\text { factors (translated) }\end{array}$} & $\begin{array}{l}\text { Optimal upfront } \\
\text { training policy } \\
\left(F A P^{*}\right)\end{array}$ & $\begin{array}{l}\text { The optimal policy was } \\
\text { searched within the range: } \\
\{F A P-0-F A P-5\} \text { in } \\
\text { increments of } 0.5 \text { times the } \\
\text { standard-time number of units } \\
\left(0.5 \times n_{s}\right) \text {. }\end{array}$ & $\begin{array}{l}\text { An upfront training } \\
\text { policy of } \\
F A P-0 / 1 / 2 \text { was } \\
\text { used in Jaber et al. } \\
\text { (2003). Model } C \\
\text { includes the effect of } \\
\text { further upfront training. }\end{array}$ \\
\hline & $\begin{array}{l}\text { Optimal centralization } \\
\text { of control policy (batch } \\
\text { transfer policy) }\left(C^{*}\right)\end{array}$ & $\begin{array}{l}\text { The optimal policy was } \\
\text { searched within the range: } \\
\{10-250 \text { units }\} \text { in } \\
\text { increments of one unit. }\end{array}$ & $\begin{array}{l}\text { Same as in model } A \text {, see } \\
\text { Table } 3.1 \text {. }\end{array}$ \\
\hline
\end{tabular}




\section{Choice of experimental design}

Model B differs from model A because the assumption of no task similarity has been released to include tasks that are similar by varying degrees. The magnitude of the effect of the similarity factor on the forgetting losses of the DPLFM is compared to the results of Jaber et al. (2003), where the LFCM was used. The implications of the results of model B are also discussed as they pertain to five possible DRC shop performance issues discussed by Hottenstein and Bowman (1998). As in model A, the analysis in model B is predominantly qualitative.

\subsubsection{Modeling methodology}

The modelling methodology is the same as in model A except that the assumption of no task similarity was released to include tasks that are similar by varying degrees.

\subsection{Experimental design of model $\mathrm{C}$}

In model $\mathrm{C}$, two multivariate functions were found that provide the optimal upfront training policy $\left(F A P^{*}\right)$ and the optimal centralization of control policy $\left(C^{*}\right)$ (batch transfer frequency) as output variables to the LFCM model $(N=2)$ given four prescribed DRC system factors: task similarity factor $(S)$, initial processing time to standard time ratio of the task $(\rho)$, worker learning rate $(L R)$, and worker forgetting rate $(F R)$.

\subsubsection{Recognition and statement of the problem}

The problem statement of model $\mathrm{C}$ is as follows: Given a degree of job similarity $(s)$, initial processing time to standard time ratio of the task $(\rho)$, and the learning and forgetting rate of the worker ( $L R, F R)$, what should the upfront training policy $\left(F A P^{*}\right)$ and centralization of control policy $\left(C^{*}\right)$ (batch transfer frequency) be in order to minimize the performance measures of average processing (service) time over approximately 3000 jobs per station? 
The results of the experiment in model $\mathrm{C}$ may prove important from a managerial perspective because it suggests preferred settings for controllable industrial DRC factors (training and transfer policies) given existing factors that cannot be as easily changed (job similarity, tasktimes, and worker learning-forgetting rate). A numerical example is included to illustrate the practicality of the function developed in model $\mathrm{C}$.

\subsubsection{Choice of factors, levels, and ranges}

The aforementioned problem of model $\mathrm{C}$ uses the following input and translated ${ }^{\mathrm{a}}$ output factors as given in Table 3.5. The experiment in model $\mathrm{C}$ searches for an optimal level of input factors given a fixed set of output factor ranges. The result yields two multivariate functions of the following form (the asterisks on $F A P$ and $C$ denote optimized values of these parameters):

$$
g(s, \rho, L R, F R)=C^{*}
$$

and

$$
g(s, \rho, L R, F R)=F A P^{*}
$$

where the input variables $(s, \rho, L R, F R)$, optimal output variables $\left(F A P^{*}, C^{*}\right)$, their levels/ranges, and justifications for these levels, are described in Table 3.5.

\subsubsection{Selection of the response variables}

The response variable in model $\mathrm{C}$ is the same as in model $\mathrm{A}$ and model $\mathrm{B}$.

\subsubsection{Choice of experimental design}

Model C is an optimization experiment that searches for the optimal set of output experimental factors (see Table 3.5) based on prescribed input parameters that minimize the response variable of average processing (service) time ( $A P T$ ) calculated over approximately 3000 jobs per station, the same performance measure used in model $A$ of this thesis and Jaber et al. (2003).

"each minimized $A P T$ value corresponded (and was translated) to a specific value for $C^{*}$ and $F A P^{*}$ 


\subsubsection{Modeling methodology}

The modelling methodology is the same as in model A and model B. The multivariate function is fitted from the table of optimized $C^{*}$ and $F A P^{*}$ values. Model $\mathrm{C}$ is a 5 (levels of task similarity) $\times 5$ (initial processing time to standard time ratio) $\times 5$ (worker learning rate) $\times 5$ (worker forgetting rate) model yielding 625 pairs of output: $C^{*}$ and $F A P^{*}$ values. Due to the distribution of the output variables described in Chapter 4, the functions in (3.10) and (3.11) were fitted to the values using binary logistic multiple-regression; these functions were then used to translate the input factors directly into optimal settings for $C$ and $F A P$ based on minimized $A P T$ values. Since these functions were fitted between data points, every optimal setting of $C$ and $F A P$ can be found for input factors throughout the continuous range of the input factors listed in Table 3.5 .

\subsection{Chapter 3 summary}

The models in this thesis do not contain randomness in their design. However, they all conduct a mathematical test in which changes are made to the input variables that result in changes to output responses, and hence, this thesis does use a type of deterministic experiment in all of its models. Due to the complexity of the output response of all the models, following an outline of an experimental design aids interpretation despite the fact that the models are deterministic. The previous chapter 3 has outlined the first four steps of experimental design mentioned by Montgomery (1997) for each model in this thesis: (1) recognition of, and statement of the problem, (2) choice of factors, levels, and ranges, (3) selection of the response variable, and, (4) choice of experimental design for all of the models with the addition of the description of the modelling methodology. The next two steps, (5) performing the experiments, and, (6) analysis (deterministic) of the data are summarized for each model in the following chapter 4. 


\section{Chapter 4: results and analysis}

\subsection{Model A results}

Appendix section 7.2.1 contains the tables of results for both levels of worker flexibility for the DPLFM. The LFCM and the VRVF model results are given for comparison in appendix section 7.2.2. The following analysis is based on these results. As previously mentioned, these results for the DPLFM were obtained from calculations performed using an Excel spreadsheet. Any necessary programming was performed using Excel-Visual Basic.

The analyses of the results of model $A$ have been subdivided into two sections: the main effect analysis and the interaction effect analysis. These two terms have been taken from the discipline of experimental design and their methods have been used in this thesis to enrich the analysis of model $\mathrm{A}$. The main effect can be defined as the total change in response or output of one factor by changing the level of the factor from its highest level to its lowest level (Montgomery, 1997). In model $\mathrm{A}$, the main effect of the factors was calculated for each learning-forgetting model. By finding this, the total relative direct influence of each factor is given quantitatively, and hence, is easily compared to the main effect of the other factors. The interaction effect is when the change in response (output) between the levels of one factor is not the same at all the levels of the other factors (Montgomery, 1997). In model A, the degree of interaction of each factor is measured quantitatively. In model $\mathrm{A}$, the interaction effect of the factors was calculated to determine the degree by which each factor interacts with each other factor. Based on the degree of these interactions, conclusions were drawn regarding the influence of these factors as they compare with the conclusions given in Jaber et al. (2003) and the five possible issues that affect DRC shop performance measures: worker flexibility, centralization of control, worker assignment, queue disciplines, and cost of transferring workers (Hottenstein and Bowman, 1998). 


\subsubsection{The main effect analysis of model $A$}

\subsubsection{Description of the main effect}

As previously mentioned, the main effect of a factor is the change in output response caused by a change in a level of the factor. In model A, this quantity is given as follows:

$$
M E_{i}=A E R_{i, \max }-A E R_{i, \min }
$$

where $M E_{i}$ is the main effect of factor $i, A E R_{i, \text { max }}$ is the average experimental response at the maximum level of factor $i$, and $A E R_{i, \min }$ is the average experimental response at the minimum level of factor $i$. Note that the experimental response in model $\mathrm{A}$ is the performance measure of average processing (service) time ( $A P T$ ) calculated over approximately 3000 jobs per station. The main effect results for the VRVF, the LFCM, and the DPLFM are discussed in the subsequent sections.

As shown in Figure 4.1, the relative main effect graphs have percentage quantity measures; they are used to compare the magnitude of the main effect of each factor as a percentage of the maximum range of output values for the model. These percentage values are calculated as follows:

$$
R M E_{i}=\frac{M E_{i}}{M A X_{j}} \times 100
$$

where $R M E_{i}$ is the relative main effect of factor $i, M E_{i}$ is the main effect of factor $i$, and $M A X_{j}$ is the maximum range of output values for model $j$. For example, Figure 4.1(c) illustrates that the relative main effect of the upfront training policy $(F A P)$ factor is more than three times as influential as the number of tasks learned $(N)$ factor (13.0\% versus $4.3 \%)$. The changes in the relative main effect between models are shown in Figure 4.2. These values are used to compare the change in the influence of one factor between models. These values are calculated as follows:

$$
\Delta R M E_{i, a, b}=R M E_{i, b}-R M E_{i, a}
$$

where $\triangle R M E_{i, a, b}$ is the change in the relative main effect of factor $i$ from learning-forgetting model $a$ to learning-forgetting model $b, R M E_{i, a}$ is the relative main effect of 


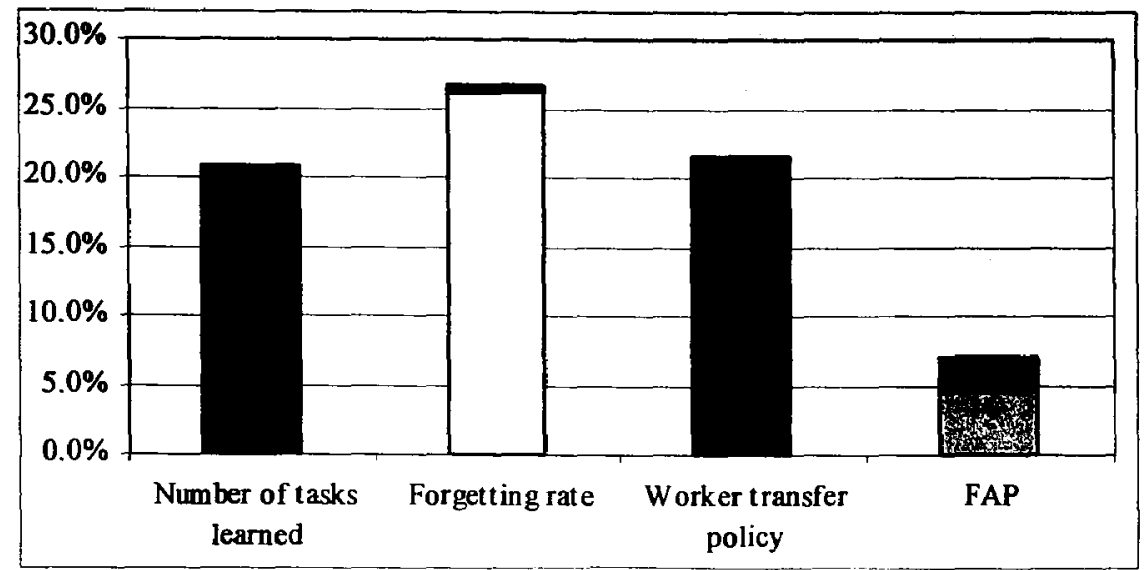

(a): The relative main effect of the factors - VRVF

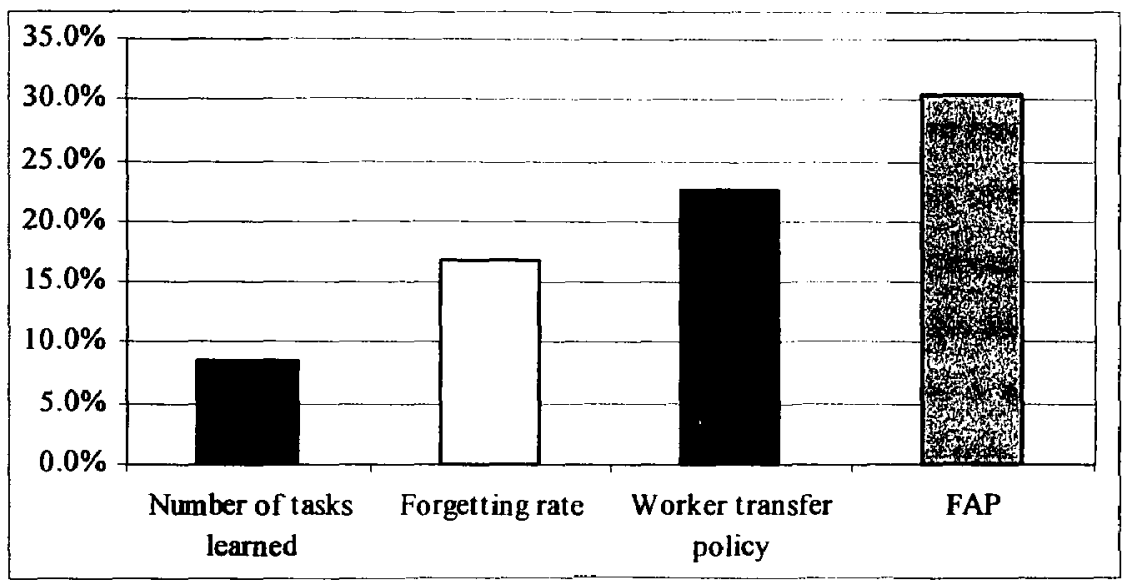

(b): The relative main effect of the factors - LFCM

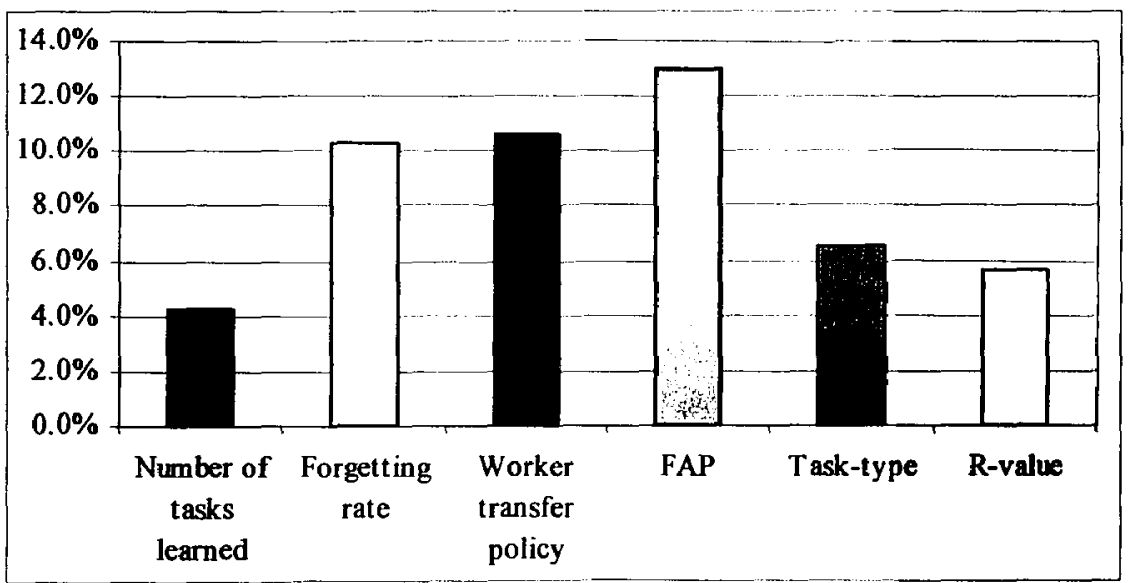

(c): The relative main effect of the factors - DPLFM

Figure 4.1: Graphs displaying the relative main effects in each learning-forgetting model 


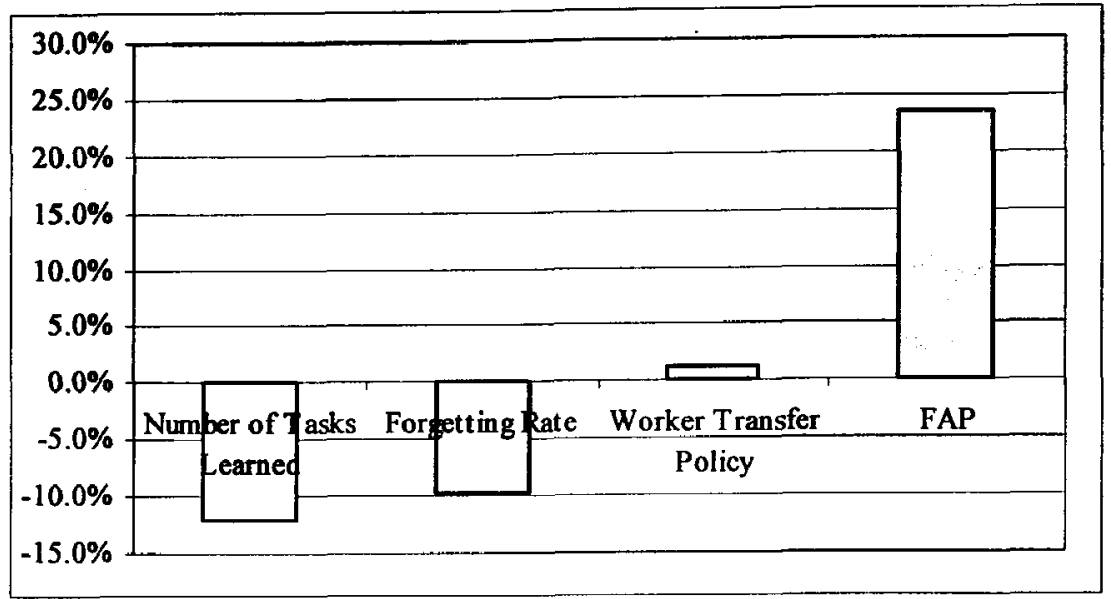

(a): Change in the relative main effect from the VRVF to the LFCM

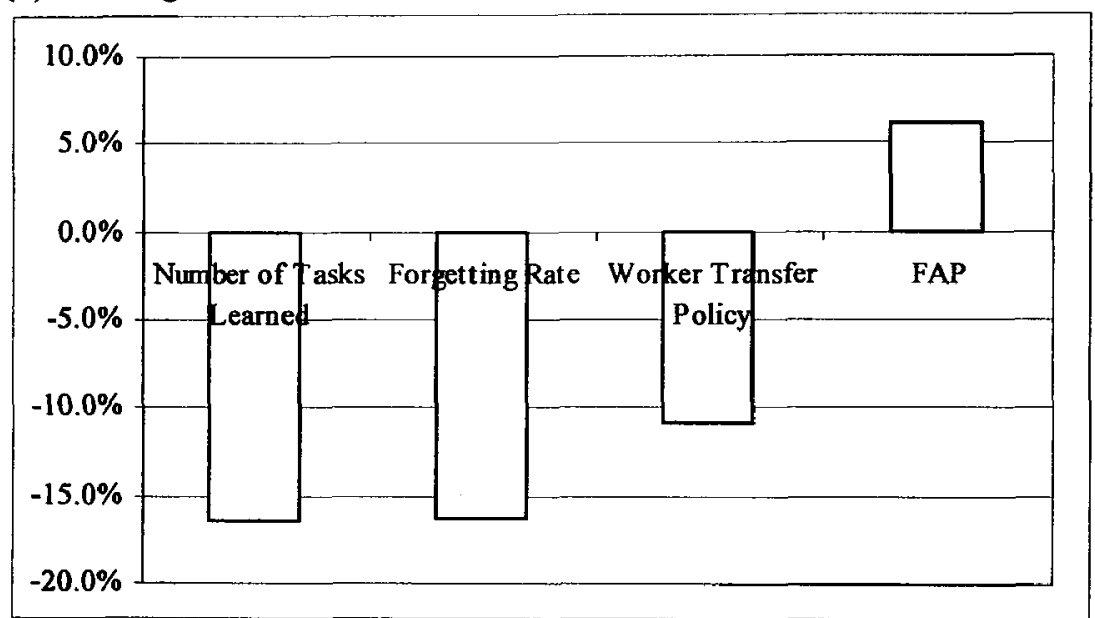

(b): Change in the relative main effect from the VRVF to the DPLFM

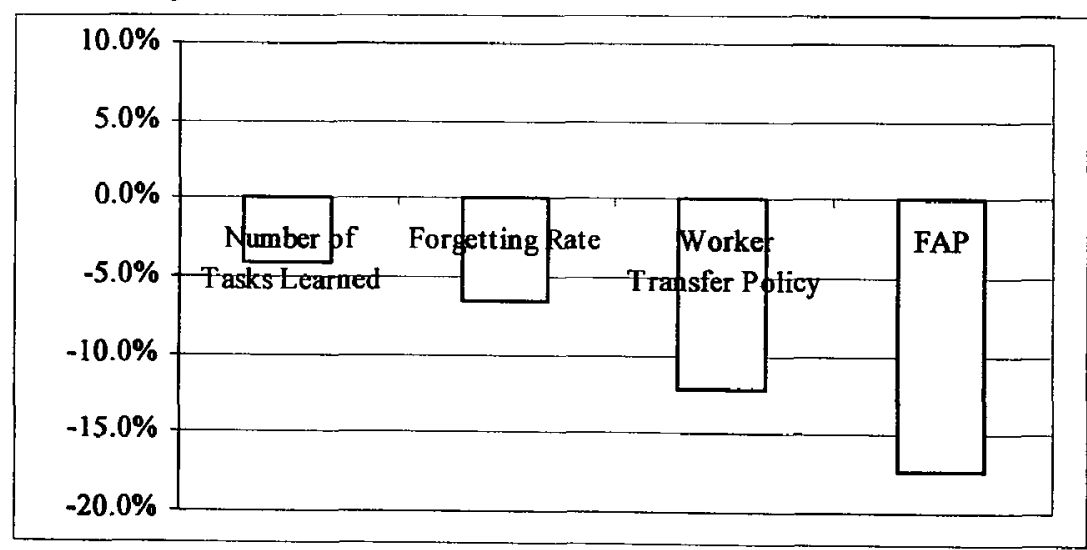

(c): Change in the relative main effect from the LFCM to the DPLFM

Figure 4.2: Changes in the relative main effect between learning-forgetting models 
factor $i$ in model $a$, and $R M E_{i, b}$ is the relative main effect of factor $i$ in model $b$.

For example, Figure 4.2(c) illustrates that the effect of the $F A P$ (upfront training policy) factor is approximately $17.5 \%$ less influential $(-17.5 \%)$ in the DPLFM than in the LFCM.

\subsubsection{Discussion of the main effect (the VRVF, the LFCM, and the DPLFM)}

\subsection{Main effect - VRVF}

As shown in Figure 4.1(a), the relative main factor effects of the VRVF are ranked in decreasing effect as follows: (1) forgetting rate, (2) worker transfer policy, (3) number of tasks learned, and (4) upfront training policy ( $F A P$ ). The relative change in performance between factor levels in the VRVF is shown in Table 4.1. For example, changing from three to two tasks learned in the VRVF causes a $19.8 \%$ increase (performance increases are denoted by a negative sign '-' because of a percent decrease in forgetting losses) in performance (second row, fourth column).

The most notable main effect in the VRVF is that the forgetting rate ranks first in importance (see Figure 4.1(a)) whereas in the LFCM it only ranks second last (third) (see Figure 4.1(b)), and only third in the DPLFM (see Figure 4.1(c)). The significance of this finding is beneficial since the worker forgetting rate factor is less controllable than the other factors.

\subsection{Main effect - LFCM}

As shown in Figure 4.1(b), the relative main factor effects of the LFCM are ranked in decreasing significance as follows: (1) upfront training policy ( $F A P)$, (2) worker transfer policy, (3) forgetting rate, and (4) number of tasks learned. The relative change in performance between factor levels in the LFCM is shown in Table 4.2. The most notable main effect in the LFCM is the large increase in relative influence of the main effect of the upfront training policy factor ( $F A P$ ) over that of the VRVF. This is consistent with the findings of Jaber et al. (2003). In fact, the ranking increases from last to first, as shown in the change between Figure 4.1(a) to Figure 4.1(b). The relative change is over 23.5\%, as shown in Figure 4.2(a). The relative influence of the worker transfer policy also increases slightly by $1.2 \%$ over the VRVF, 
Table 4.1: The main effect of the factors in the VRVF

\begin{tabular}{|c|c|c|c|}
\hline $\begin{array}{l}\text { Number of } \\
\text { tasks learned } \\
(N)\end{array}$ & $A P T$ & Level change & $\begin{array}{l}\text { Relative \% } \\
\text { change in the } \\
\text { main effect } \\
\text { between levels }\end{array}$ \\
\hline 2 & 1.0670 & \multirow{3}{*}{$\begin{array}{l}3 \text { tasks learned } \\
\text { to } 2 \text { tasks } \\
\text { learned }\end{array}$} & \multirow{3}{*}{$-19.8 \%$} \\
\hline 3 & 1.3302 & & \\
\hline Main effect & 0.2632 & & \\
\hline $\begin{array}{l}\text { Forgetting rate } \\
(F R)\end{array}$ & $A P T$ & Level change & $\begin{array}{l}\text { Relative \% } \\
\text { change in the } \\
\text { main effect } \\
\text { between levels }\end{array}$ \\
\hline $95 \%$ (low) & 1.0403 & $90 \%$ to $95 \%$ & $-11.6 \%$ \\
\hline $90 \%$ (med) & 1.1775 & $85 \%$ to $90 \%$ & $-14.6 \%$ \\
\hline $85 \%$ (high) & 1.3781 & & \\
\hline Main effect & 0.3378 & $85 \%$ to $95 \%$ & $-24.5 \%$ \\
\hline $\begin{array}{l}\text { Worker } \\
\text { transfer policy } \\
(C)\end{array}$ & $A P T$ & Level change & $\begin{array}{l}\text { Relative \% } \\
\text { change in the } \\
\text { main effect } \\
\text { between levels }\end{array}$ \\
\hline 10 & 1.3253 & 10 to 25 & $-8.1 \%$ \\
\hline 25 & 1.2185 & 25 to 250 & $-13.7 \%$ \\
\hline 250 & 1.0521 & & \\
\hline Main effect & 0.2731 & 10 to 250 & $-20.6 \%$ \\
\hline $\begin{array}{l}\text { Upfront } \\
\text { training policy } \\
(F A P)\end{array}$ & $A P T$ & Level change & $\begin{array}{l}\text { Relative \% } \\
\text { change in the } \\
\text { main effect } \\
\text { between levels }\end{array}$ \\
\hline 0 & 1.2564 & $F A P-0$ to $F A P-1$ & $-6.8 \%$ \\
\hline 1 & 1.1714 & $F A P-1$ to $F A P-2$ & $-0.3 \%$ \\
\hline 2 & 1.1681 & & \\
\hline Main effect & 0.0883 & $F A P-0$ to $F A P-2$ & $-7.0 \%$ \\
\hline
\end{tabular}


Table 4.2: The main effect of the factors in the LFCM

\begin{tabular}{|c|c|c|c|}
\hline $\begin{array}{l}\text { Number of } \\
\text { tasks learned } \\
(N)\end{array}$ & $A P T$ & Level change & $\begin{array}{l}\text { Relative \% } \\
\text { change in the } \\
\text { main effect } \\
\text { between levels }\end{array}$ \\
\hline 2 & 1.1660 & \multirow{3}{*}{$\begin{array}{l}3 \text { tasks learned } \\
\text { to } 2 \text { tasks } \\
\text { learned }\end{array}$} & \multirow{3}{*}{$-9.7 \%$} \\
\hline 3 & 1.2919 & & \\
\hline Main effect & 0.1259 & & \\
\hline $\begin{array}{l}\text { Forgetting rate } \\
(F R)\end{array}$ & $A P T$ & Level change & $\begin{array}{l}\text { Relative \% } \\
\text { change in the } \\
\text { main effect } \\
\text { between levels }\end{array}$ \\
\hline $95 \%$ (low) & 1.1026 & $90 \%$ to $95 \%$ & $-10.6 \%$ \\
\hline $90 \%$ (med) & 1.2331 & $85 \%$ to $90 \%$ & $-8.7 \%$ \\
\hline $85 \%$ (high) & 1.3513 & & \\
\hline Main effect & 0.2487 & $85 \%$ to $95 \%$ & $-18.4 \%$ \\
\hline $\begin{array}{l}\text { Worker } \\
\text { transfer policy } \\
\text { (C) }\end{array}$ & $A P T$ & Level change & $\begin{array}{l}\text { Relative \% } \\
\text { change in the } \\
\text { main effect } \\
\text { between levels }\end{array}$ \\
\hline 10 & 1.3898 & 10 to 25 & $-10.6 \%$ \\
\hline 25 & 1.2427 & 25 to 250 & $-15.2 \%$ \\
\hline 250 & 1.0545 & & \\
\hline Main effect & 0.3353 & 10 to 250 & $-24.1 \%$ \\
\hline $\begin{array}{l}\text { Upfront } \\
\text { training policy } \\
(F A P)\end{array}$ & $A P T$ & Level change & $\begin{array}{l}\text { Relative \% } \\
\text { change in the } \\
\text { main effect } \\
\text { between levels }\end{array}$ \\
\hline 0 & 1.5290 & $F A P-0$ to $F A P-1$ & $-29.4 \%$ \\
\hline 1 & 1.0791 & $F A P-1$ to $F A P-2$ & $-0.02 \%$ \\
\hline 2 & 1.0789 & & \\
\hline Main effect & 0.4501 & $F A P-0$ to $F A P-2$ & $-29.4 \%$ \\
\hline
\end{tabular}


whereas the relative influence of the forgetting rate and the number of tasks learned decreased by $9.8 \%$ and $12.2 \%$ respectively (see Figure $4.2(\mathrm{a})$ ). Again, the significance of this finding is beneficial because if learning and forgetting is assumed to more closely follow the LFCM than the VRVF, there is added consolation to DRC job shop practitioners that more controllable factors such as the upfront training policy $(F A P)$ and the worker transfer policy $(C)$ are more significant than less controllable factors such as the worker forgetting rate $(F R)$ and the number of tasks learned $(N)$.

\subsection{Main effect - DPLFM}

As shown in Figure 4.1(c), the relative main factor effects of the DPLFM are ranked in decreasing effect as follows: (1) upfront training policy $(F A P)$, (2) worker transfer policy $(C)$, (3) forgetting rate, (4) task-type, (5) the $R$-value, and, (6) the number of tasks learned.

The relative change in performance between factor levels in the DPLFM is shown in Table 4.3. Note the two additional DPLFM experimental factors of $R$-value and task-type in the first column of Table 4.3. There are several notable main effect observations in the DPLFM. Firstly, the overall detriment of learning three tasks is less pronounced in the DPLFM than in the other models (see the second row, fourth column of Table 4.3 compared with the same cell of Table 4.1 and Table 4.2). Secondly, the benefit of providing the additional upfront training of $F A P-2$ over just $F A P-1$ upfront training is slightly more evident in the DPLFM than in the LFCM, but slightly less evident than in the VRVF (see row 17, column four of Table 4.3 compared with the same cell of Table 4.1 and Table 4.2). Thirdly, overall, upfront training (either FAP-1 or FAP-2) in the DPLFM reduces forgetting losses more than it did in the VRVF but less than in the LFCM (see row 19, column four of Table 4.3 compared with the same cell of Table 4.1 and Table 4.2). This relative change in the influence of upfront training from the VRVF and the LFCM to the DPLFM is also illustrated in Figure 4.2(b) and Figure 4.2(c) respectively. Jaber et al. (2003) deduced that since: (1) the LFCM attributed more benefit to upfront training than the VRVF, and, (2) a greater benefit is assumed by providing upfront training to workers performing dissimilar tasks, that the LFCM more closely models a job shop that performs dissimilar tasks. By this reasoning, it is deduced that the aforementioned main effect results of the DPLFM suggest that the DPLFM accrues less importance on the dissimilarity of the tasks being performed than the LFCM. Model B of this thesis addresses this issue. In addition, as shown in Figure 4.2(c), the inclusion of additional factors in the DPLFM ( $R$-value and task-type) caused the reduction of the relative influence of all the factors 
Table 4.3: The main effect of the factors in the DPLFM

\begin{tabular}{|c|c|c|c|}
\hline $\begin{array}{l}\text { Number of tasks } \\
\text { learned }(N)\end{array}$ & $A P T$ & Level change & $\begin{array}{l}\text { Relative \% change } \\
\text { in the main effect } \\
\text { between levels }\end{array}$ \\
\hline 2 & 1.0722 & \multirow{3}{*}{$\begin{array}{l}3 \text { tasks learned to } 2 \\
\text { tasks learned }\end{array}$} & \multirow{3}{*}{$-6.2 \%$} \\
\hline 3 & 1.1435 & & \\
\hline Main effect & 0.0712 & & \\
\hline $\begin{array}{l}\text { Forgetting rate } \\
(F R)\end{array}$ & $A P T$ & Level change & $\begin{array}{l}\text { Relative \% change } \\
\text { in the main effect } \\
\text { between levels }\end{array}$ \\
\hline $95 \%$ (low) & 1.0261 & $90 \%$ to $95 \%$ & $-6.8 \%$ \\
\hline $90 \%$ (med) & 1.1006 & $85 \%$ to $90 \%$ & $-8.0 \%$ \\
\hline $85 \%$ (high) & 1.1968 & & \\
\hline Main effect & 0.1707 & $85 \%$ to $95 \%$ & $-14.3 \%$ \\
\hline $\begin{array}{l}\text { Worker transfer } \\
\text { policy }(C)\end{array}$ & $A P T$ & Level change & $\begin{array}{l}\text { Relative \% change } \\
\text { in the main effect } \\
\text { between levels }\end{array}$ \\
\hline 10 & 1.2022 & 10 to 25 & $-9.0 \%$ \\
\hline 25 & 1.0937 & 25 to 250 & $-6.0 \%$ \\
\hline 250 & 1.0276 & & \\
\hline Main effect & 0.1746 & 10 to 250 & $-14.5 \%$ \\
\hline $\begin{array}{l}\text { Upfront training } \\
\text { policy }(F A P)\end{array}$ & $A P T$ & Level change & $\begin{array}{l}\text { Relative \% change } \\
\text { in the main effect } \\
\text { between levels }\end{array}$ \\
\hline 0 & 1.2507 & $F A P-0$ to $F A P-1$ & $-17.1 \%$ \\
\hline 1 & 1.0369 & $F A P-1$ to $F A P-2$ & $-0.1 \%$ \\
\hline 2 & 1.0358 & & \\
\hline Main effect & 0.2149 & $F A P-0$ to $F A P-2$ & $-17.2 \%$ \\
\hline Task-type & $A P T$ & Level change & $\begin{array}{l}\text { Relative \% change } \\
\text { in the main effect } \\
\text { between levels }\end{array}$ \\
\hline$T_{C}$ & 1.0643 & $T_{M}$ to $T_{C}$ & $-9.2 \%$ \\
\hline$T_{M}$ & 1.1718 & $T_{M}$ to $T_{\text {Med }}$ & $-5.5 \%$ \\
\hline$T_{\text {Med }}$ & 1.1070 & $T_{M}$ to $T_{U}$ & $-7.1 \%$ \\
\hline \multirow[t]{2}{*}{$T_{U}$} & 1.0884 & $T_{M e d}$ to $T_{c}$ & $-3.9 \%$ \\
\hline & & $T_{U}$ to $T_{C}$ & $-2.2 \%$ \\
\hline Main effect & 0.1075 & $T_{M}$ to $T_{C}$ & $-9.2 \%$ \\
\hline$R$ & $A P T$ & Level change & $\begin{array}{l}\text { Relative \% change } \\
\text { in the main effect } \\
\text { between levels }\end{array}$ \\
\hline $1 / 3$ & 1.1553 & $1 / 3$ to 1 & $-4.2 \%$ \\
\hline 1 & 1.1067 & 1 to 3 & $-4.1 \%$ \\
\hline 3 & 1.0615 & & \\
\hline Main effect & 0.0938 & $1 / 3$ to 3 & $-8.1 \%$ \\
\hline
\end{tabular}


common to all three models (number of tasks learned, forgetting rate, worker transfer policy, and $F A P$ ) over that of the LFCM, with the main effect of $F A P$ being reduced the most. As expected, the performance change between the levels of performing a motor task $\left(T_{M}\right)$ to performing a cognitive task $\left(T_{C}\right)$ is a main effect change in the DPLFM because both tasks are found on opposite extremes of the range of learning slopes used in this thesis (see row 26, column four of Table 4.3). Also, it is interesting to note that, overall, the performance of task $T_{U}$ yields less forgetting losses $(A P T=1.0884$, see row 24, column two of Table 4.3) than $T_{\text {Med }}(A P T=1.1070$, see row 23 , column two of Table 4.3), a task with an average learning rate equal to that of $T_{U}$, and nearly as little forgetting losses as task $T_{C}(A P T=1.0643$, see row 21, column two of Table 4.3). Consequently, it could be inferred that forgetting losses may be underestimated when the dual phase cognitive and motor task elements are not considered. Finally, it is of importance to note that the main effect of the $R$-value ranks second last in the DPLFM. However, the interaction effect of the $R$-value is significant, as shown in the following interaction effect section.

\subsubsection{Main effect conclusions}

From a managerial perspective, the following three findings of the main effect analysis should be noted: (1) the effectiveness of reducing forgetting losses in a DRC job shop may be more achievable by adjusting the controllable factors such as upfront training policy and worker transfer policy than by focusing on uncontrollable factors such as the worker forgetting rate (the LFCM versus the VRVF); this finding is used to develop the reasoning for model $\mathrm{C}$ of this thesis, (2) the results of the main effect of the DPLFM suggest that increasing the number of tasks learned beyond two may be more achievable in a DRC context than suggested by the LFCM; (3) the type of worker-task interaction with regards to the learning rates of the worker on the task $(T)$ and the ratio of time for the first unit under purely cognitive conditions to time for the first unit under purely motor conditions $(R)$ may be more of an issue than the number of tasks for which the worker is trained (the DPLFM versus the LFCM, see Figure 4.1(c) and Figure 4.1(b)), and (4) the findings of model A suggest that forgetting losses may be underestimated when the dual phase cognitive and motor task elements are not considered, and as a result, the task-type factor inherent to the DPLFM is a factor that may be important to include when examining further DRC forgetting loss reductions.

It is of interest to note that finding (2) suggests that research pursuing a quantitative trade-off 
between forgetting loss reduction and job flexibility may be of significance. However, a quantitative measure of shop flexibility would be required in order for a measurable trade-off analysis.

\subsubsection{The interaction effect analysis of model $A$}

\subsubsection{Description of the interaction effect}

As previously mentioned, the interaction effect of a factor is when the change in response (output) between the levels of one factor is not the same at all the levels of the other factors. In model $\mathrm{A}$, the magnitude of the interaction effect between factor levels was calculated by subdividing the results table into sub-tables forming all the possible 2-way interactions between factors. For simplification, the factors were given acronyms. These acronyms and the 2-way factor interactions are listed in Table 4.4. The 15 non-duplicated interactions are underlined.

For example, Table 4.4(a) illustrates a sub-table consisting of averaged results for each worker transfer policy level - forgetting rate level pair. The values are averaged irrespective of the levels of any other factor. As a result, this is referred to as a 2-way interaction table $C-F R$, with $C$ referring to the centralization of control policy (worker transfer policy) and $F R$ referring to the worker forgetting rate. Since there were a total of 152 -way factor interactions there were 152 -way interaction tables constructed. All of the 2-way interaction tables are given in the appendix section 7.3. A baseline measure was required to determine the magnitude of the interaction at each factor level. This was done by measuring the relative percentage difference of each interaction pair with the lowest interaction pair in the table (professor Gharghouri, Dr. Kolasa, and Dr. Todorow, personal communication, 2003). Table 4.4(b) illustrates how the table looks after this is done. For example, the bolded value in Table 4.4(b) represents the baseline pair $C_{250} F R_{95 \%}$ (referring to a batch transfer size of 250 with a worker forgetting rate of $95 \%$ ) since this pair has the lowest averaged result in Table 4.4(a) (see 1.014 and $0.0 \%$ in row five, column three of Table 4.4(a) and Table 4.4(b)). All the other 2-way factor level interactions are shown by their relative difference above the baseline measure. For example, in Table 4.4(b), the 2-way point interaction of $C_{25} F R_{90 \%}$ is $6.5 \%$ above the baseline measure. Also, only a relative change of $6 \%$ or greater between any 2-way point interaction is considered a significant change. 
Table 4.4: Factors, their acronyms, and their interactions for model $A$

\begin{tabular}{|l|l|l|}
\hline Factor & Acronym & 2-Way interactions \\
\hline $\begin{array}{l}\text { Worker transfer } \\
\text { policy }\end{array}$ & $C$ & $\frac{C-F R, C-T, C-F A P, C-N, C-R}{F R-T, F R-F A P, F R-N, F R-R, F R-C}$ \\
\hline Forgetting rate & $F R$ & $\frac{T-C, T-F R, T-F A P, T-N, T-R}{1}$ \\
\hline Task-type & $T$ & $F A P-C, F A P-F R, F A P-T, F A P-N, F A P-R$ \\
\hline $\begin{array}{l}\text { Upfront training } \\
\text { policy }\end{array}$ & $F A P$ & $N-C, N-F R, N-T, N-F A P, N-R$ \\
\hline $\begin{array}{l}\text { Number of tasks } \\
\text { Learned }\end{array}$ & $N$ & $R-C, R-F R, R-T, R-F A P, R-N$ \\
\hline$R$-value & $R$ &
\end{tabular}

Table 4.4(a): 2-way interaction

\begin{tabular}{|l|l|l|l|l|}
\hline \multicolumn{2}{|c|}{} & \multicolumn{3}{|l|}{ Forgetting rate $(F R)$} \\
\cline { 3 - 5 } \multicolumn{2}{|c|}{} & $\mathbf{9 5 \%}$ & $\mathbf{9 0 \%}$ & $\mathbf{8 5 \%}$ \\
\hline $\begin{array}{l}\text { Worker } \\
\text { transfer policy } \\
(C)\end{array}$ & $\mathbf{1 0}$ & 1.044 & 1.198 & 1.365 \\
\cline { 2 - 5 } & $\mathbf{2 5}$ & 1.020 & 1.080 & 1.181 \\
\cline { 2 - 5 } & $\mathbf{2 5 0}$ & $\mathbf{1 . 0 1 4}$ & 1.024 & 1.045 \\
\hline
\end{tabular}

Table 4.4(b): 2-way interaction with relative difference from baseline

\begin{tabular}{|l|l|l|l|l|}
\hline \multicolumn{2}{|c|}{} & \multicolumn{4}{|l|}{ Forgetting rate $(F R)$} \\
\cline { 3 - 5 } \multicolumn{2}{|c|}{} & $\mathbf{9 5 \%}$ & $\mathbf{9 0 \%}$ & $\mathbf{8 5 \%}$ \\
\hline $\begin{array}{l}\text { Worker } \\
\text { transfer policy } \\
(C)\end{array}$ & $\mathbf{1 0}$ & $2.9 \%$ & $18.1 \%$ & $34.6 \%$ \\
\cline { 2 - 5 } & $\mathbf{2 5}$ & $0.6 \%$ & $6.5 \%$ & $16.4 \%$ \\
\cline { 2 - 5 } & $\mathbf{2 5 0}$ & $\mathbf{0 . 0 \%}$ & $1.0 \%$ & $3.0 \%$ \\
\hline
\end{tabular}

Table 4.5(a): 3-way interaction for $C_{10} F R_{85 \%}$

\begin{tabular}{|l|c|c|c|c|c|c|c|c|c|c|c}
\hline \multicolumn{2}{|l|}{$C_{10} F R_{85 \%}$} \\
\hline$T$ & & $\%$ & $F A P$ & & $\%$ & $N$ & & $\%$ & $R$ & & $\%$ \\
\hline$T_{C}$ & 1.251 & $23.3 \%$ & 0 & 1.884 & $85.7 \%$ & 2 & 1.254 & $23.6 \%$ & $1 / 3$ & 1.442 & $42.1 \%$ \\
\hline$T_{M}$ & 1.493 & $47.2 \%$ & 1 & 1.135 & $11.9 \%$ & 3 & 1.476 & $45.5 \%$ & 1 & 1.376 & $35.7 \%$ \\
\hline$T_{M e d}$ & 1.368 & $34.9 \%$ & 2 & 1.076 & $6.1 \%$ & & & & 3 & 1.302 & $28.3 \%$ \\
\hline$T_{U}$ & 1.346 & $32.7 \%$ & & & & & & & & & \\
\hline
\end{tabular}

Table 4.5(b): 3-way interaction for $C_{250} F R_{95 \%}$

\begin{tabular}{|l|c|c|c|c|c|c|c|c|c|c|c|}
\hline \multicolumn{2}{|l|}{$C_{250} F R_{95 \%}$} \\
\hline$T$ & & $\%$ & $F A P$ & & $\%$ & $N$ & & $\%$ & $R$ & & $\%$ \\
\hline$T_{C}$ & 1.009 & $-0.5 \%$ & 0 & 1.019 & $0.5 \%$ & 2 & 1.013 & $-0.1 \%$ & $1 / 3$ & 1.070 & $5.5 \%$ \\
\hline$T_{M}$ & 1.025 & $1.0 \%$ & 1 & 1.003 & $-1.1 \%$ & 3 & 1.015 & $0.1 \%$ & 1 & 1.013 & $-0.1 \%$ \\
\hline$T_{M e d}$ & 1.013 & $-0.1 \%$ & 2 & 1.021 & $0.7 \%$ & & & & 3 & 1.009 & $-0.5 \%$ \\
\hline$T_{U}$ & 1.018 & $0.4 \%$ & & & & & & & & & \\
\hline
\end{tabular}


For example, moving from a 2-way point interaction of $C_{250} F R_{90 \%}$ to $C_{10} F R_{95 \%}$ does not constitute a significant change in output response since the difference in measure from the baseline for these two values is only $1.9 \%(2.9 \%-1.0 \%)$. This base measure of $6 \%$ is consistent with the suppression measure used in Jaber et al. (2003). In addition, 3-way factor level interactions were also examined. This was done by extracting the largest two interactions (highest and lowest (baseline) performance result) within each 2-way interaction table and pairing them up against all other factors in order to search for any significant 3-way factor interactions. For example, $C_{10} F R_{85 \%}$ and $C_{250} F R_{95 \%}$ in Table 4.4(b) exhibited the largest and smallest (baseline) performance interaction (34.6\% and $0.0 \%$ ) results respectively within all the levels of the $C-F R$ interactions table. As a result, $C_{10} F R_{85 \%}$ and $C_{250} F R_{95 \%}$ were then paired up against all the other factors to form additional 3-way interaction sub-tables. It was assumed that only significant 2-way interactions would foster any significant 3-way interactions (Dr. Gharghouri and Dr. Todorow, personal communication, 2003). Examples of 3-way interaction tables for $C_{10} F R_{85 \%}$ and $C_{250} F R_{95 \%}$ are given in Table 4.5(a) and Table 4.5(b). The method for determining whether there were any significant 3-way interactions is given by listing maximum and minimum interaction criterion measures. These are given as follows:

\section{Upper interaction criterion:}

$<$ (second highest 2-way interaction) $-6.0 \%$

\section{Lower interaction criterion:}

$$
>\text { (second lowest 2-way interaction) }+6.0 \% \text {. }
$$

For example, for the 3-way interaction table in Table 4.5(a), the upper interaction criterion is calculated as follows:

$$
\text { Upper interaction criterion }<(18.1 \%)-6.0 \%=12.1 \% \text {, }
$$

where the second highest 2-way interaction (18.1\%) refers to the value in the third row, fourth column of Table $4.4(\mathrm{~b})$. The $6 \%$ is used as a buffer value; this is consistent with the suppression measure used in Jaber et al. (2003). As shown in the shaded cells of Table 4.5(a), the 3-way interactions $C_{10} F R_{85 \%} F A P-1$ and $C_{10} F R_{85 \%} F A P-2$ are significant 3-way interactions because they are less than the upper interaction criterion: $(11.9 \%, 6.1 \%)<12.1 \%$. The implications of significant 3-way interactions are that they provide exceptions to the general 
conclusions made concerning the 2-way interactions.

Also, for the 3-way interaction table in Table 4.5(b), the lower interaction criterion is calculated as follows:

$$
\text { Lower interaction criterion }>(0.6 \%)+6.0 \%=6.6 \% \text {, }
$$

where the second lowest 2-way interaction $(0.6 \%)$ refers to the value in the fourth row, third column of Table 4.4(b). However, in this particular case, as shown in Table 4.5(b), there are no shaded cells depicting significant 3-way interactions because all the 3-way interactions are less than the lower interaction criterion of $6.6 \%$.

All the 3-way interaction tables are given in the appendix section 7.4. The conclusions drawn from these 2-way and 3-way factor interactions are compared to the conclusions of Jaber et al. (2003) and to the five possible issues that affect DRC shop performance measures: worker flexibility, centralization of control, worker assignment, queue disciplines, and cost of transferring workers given by Hottenstein and Bowman (1998).

\subsubsection{Discussion of the interaction effect (LFCM and DPLFM)}

\subsection{The interactions between $C$ and $F R$}

\subsection{LFCM}

Given a worker transfer policy of 250 , varying the forgetting rate has no significant effect on performance. However, given a worker transfer policy of 10 or 25 , decreasing the amount of forgetting at any level significantly improves performance. Given a worker with a low (95\%) forgetting rate, increasing the worker transfer policy from 10 to 25 benefits performance, whereas a further increase to 250 provides no significant additional benefit. Given a forgetting rate of $90 \%$ or $85 \%$, increasing the worker transfer policy at any level improves performance. Performance is best when the worker transfer policy is 250 in a low forgetting rate (95\%). Performance is poorest when the worker transfer policy is 10 in a high forgetting rate (85\%); 
however, an exception is when the upfront training is $F A P-2$ or $F A P-1$, in these special cases forgetting losses are significantly reduced. There is no benefit in reducing the worker transfer policy regardless of the level of worker forgetting. These results contradict those of Hottenstein and Bowman (1998) who suggested that the effect of worker forgetting may cause centralized control (smaller batch transfer policy) to be much more effective than decentralized control (larger batch transfer policy).

\subsection{DPLFM}

With regards to this particular 2-way interaction of the DPLFM, the results differ from the above LFCM results by the following: (1) given a worker transfer policy of 25 , decreasing the rate of worker forgetting from $85 \%$ to $90 \%$ significantly improves performance, however, an additional reduction to $95 \%$ provides no further significant benefit, (2) given a forgetting rate of $95 \%$, increasing the worker transfer policy at any level has no significant effect on performance, (3) given a forgetting rate of $90 \%$, increasing the worker transfer policy from 10 to 25 significantly improves performance, however, an additional increase to $C=250$ provides no further significant benefit, and, (4) as in the LFCM, there is no benefit in reducing the worker transfer policy regardless of the level of worker forgetting; and hence, the results contradict those of Hottenstein and Bowman (1998). Also, the best and worst 2-way interactions and the 3-way interaction exceptions to the worst case 2-way interaction $\left(C_{10} F R_{85 \%}\right)$ are identical for the DPLFM.

\subsection{Discussion}

The results suggest that, at this interaction, the combined effect of reducing the forgetting rate and/or increasing the worker transfer policy is slightly less beneficial in reducing forgetting losses in the DPLFM than in the LFCM. This concurs with the relative main effect of the factors, see Figure 4.1(b) and Figure 4.1(c).

\subsection{The interactions between $C$ and $F A P$}

\subsection{LFCM}

Given a worker transfer policy of 250 , varying the $F A P$ level has no significant effect on performance. However, given a worker transfer policy of 10 or 25 , increasing the $F A P$ level 
from 0 to 1 improves performance, but a further increase to $F A P-2$ provide no significant additional benefits. Given a $F A P$ level of 2 , varying the worker transfer policy has no significant effect on performance. Given a $F A P$ level of 1 , increasing the worker transfer policy from 10 to 25 yields no improvement; however, an increase from 25 to 250 provides significant benefits. Given a $F A P$ level of 0 , increasing the worker transfer policy at any level provides a significant performance improvement. Performance is best when the worker transfer policy is 250 and the upfront training policy is $F A P-1$. Performance is poorest when the worker transfer policy is 10 and there is no upfront training ( $F A P-0)$. At this interaction, there are no 3-way interaction exceptions to these extreme cases.

\subsection{DPLFM}

The interactions of the DPLFM are similar to the LFCM except for the following: given $F A P$ levels of 1 or 2 , increasing the worker transfer policy at any level has no significant effect on performance, whereas in the LFCM, only FAP-2 rendered the level of the worker transfer policy level immaterial. The best and poorest 2-way interactions for performance are identical to the LFCM for this particular interaction. However, in the DPLFM, a low forgetting rate (95\%) yielded a 3-way exception that significantly reduced the harshest forgetting situation $\left(C_{10}-F A P-0-F R_{95 \%}\right)$.

\subsection{Discussion}

At this interaction, the results suggest that, since upfront training and worker transfer policies are more influential in the LFCM than the DPLFM (see Figure 4.2(c)), there is no remedy for the harshest forgetting conditions $\left(C_{10}-F A P-0\right)$ in the LFCM, whereas a reduction in worker forgetting $(95 \%)$ does significantly mitigate forgetting losses in the DPLFM.

\subsection{The interactions between $C$ and $N$}

\subsection{LFCM}

Given a worker transfer policy of 250 , varying the number of tasks learned has no significant effect on performance. However, given a worker transfer policy of 10 or 25 , decreasing the number of tasks learned from three to two provides significant benefits. Irrespective of the 
number of tasks learned, increasing the worker transfer policy at any level provides significant benefits. Performance is best when the worker transfer policy is 250 and the number of tasks learned is two. Performance is poorest when the worker transfer policy is 10 and the number of tasks learned is three; however, an exception is when the upfront training is $F A P-2$ or $F A P-1$ and/or the forgetting rate is low (95\%), in these special cases forgetting losses are significantly reduced.

\subsection{DPLFM}

With regards to this particular 2-way interaction of the DPLFM, the results are identical to the above LFCM results except for the following: when two tasks are learned in the DPLFM, increasing the worker transfer policy beyond 25 provides no further benefits whereas in the LFCM increasing $C$ was effective at any level of $N$. Also, in the DPLFM, only FAP -2 upfront training and/or a low forgetting rate (95\%) provide 3-way exceptions to the harshest forgetting losses.

\subsection{Discussion}

Again, the interaction effect of the factors that are more influential in the LFCM is evident. Namely, the upfront training factor $(F A P)$ is far more influential in the LFCM than in the DPLFM (see Figure 4.2(c)); and as a result, only a lengthy upfront training policy of $F A P-2$ is significant in reducing the harshest case of forgetting losses in the DPLFM, whereas in the LFCM just $F A P-1$ is sufficient.

\subsection{The interactions between $F R$ and $F A P$}

\subsection{LFCM}

Irrespective of the forgetting rate, increasing the upfront training policy from $F A P-0$ to $F A P-1$ provides significant benefits; however, an additional increase to $F A P-2$ provides no significant additional benefit. Given an upfront training policy of $F A P-1$ or $F A P-2$, decreasing the forgetting rate from high (85\%) to medium (90\%) provides significant benefits; however a further decrease to a low (95\%) forgetting rate provides no significant additional 
benefit. Given no upfront training $F A P-0$, decreasing the level of forgetting at any level provides improved performance. Performance is best when the forgetting rate is low (95\%) and the upfront training is $F A P-1$. Performance is poorest when the forgetting rate is high $(\mathbf{8 5} \%)$ and there is no upfront training ( $F A P-0$ ); however, an exception is when the worker transfer policy is 250 , in this special case forgetting losses are significantly reduced.

\subsection{DPLFM}

The interactions of the DPLFM differ from the previous LFCM interactions by the following: given an upfront training level of $F A P-2$, varying the level of worker forgetting does not significantly affect performance, whereas in the LFCM, a reduction in worker forgetting from $85 \%$ to $90 \%$ provides benefits at $F A P-2$. Interestingly, the highest and lowest 2-way interactions and the 3-way interaction exception for the DPLFM were identical to the above LFCM interactions.

\subsection{Discussion}

The relative effect of decreasing the forgetting rate in this interaction is shown to be slightly less in the DPLFM than in the LFCM. This is probably due to the fact that the forgetting rate factor is less influential in the DPLFM than in the LFCM (see Figure 4.2(c)). However, overall, the difference in interaction results between both learning-forgetting models is minimal at this particular interaction. Also, in both learning models, increasing the worker transfer policy to 250 can alleviate the poorest case of forgetting losses.

\subsection{The interactions between $F R$ and $N$}

\subsection{LFCM}

Given a forgetting rate of $95 \%$, varying the number of tasks learned does not significantly affect performance. However, given a forgetting rate of $90 \%$ or $85 \%$, decreasing the number of tasks learned from three to two significantly benefits performance. Irrespective of the number of tasks learned, decreasing the amount of forgetting at any level significantly benefits performance. Performance is best when the forgetting rate is low (95\%) and the number of tasks learned is two. Performance is poorest when the forgetting rate is high $(85 \%)$ and the number of tasks 
learned is three; however, an exception is when the worker transfer policy is 250 and/or the upfront training policy is $F A P-2$, in these special cases forgetting losses are significantly reduced.

\subsection{DPLFM}

The interactions of the DPLFM differ from the previous LFCM interactions by the following: when two tasks are learned $(N=2)$, only a reduction in worker forgetting from $85 \%$ to $90 \%$ provides significant benefits, whereas a further reduction to a low forgetting rate provides no significant additional benefit. This differs from the LFCM because, in the LFCM, irrespective of $N$, reducing worker forgetting at any level is beneficial. Also, in the DPLFM, only the worker transfer policy of 250 (not also $F A P-2$ as in the LFCM) is a special case 3-way interaction that significantly reduces the severest case of forgetting losses.

\subsection{Discussion}

Again, the relative influence of upfront training and the worker forgetting rate is less evident in the DPLFM than in the LFCM. This is because, in this case, the large initial training of two times the standard time was effective in reducing the severest cases of forgetting losses (3-way interaction) in the LFCM, whereas in the DPLFM, FAP-2 was ineffective. Also, in the DPLFM at $N=2$, the reduction of forgetting losses was slightly more insensitive to the level of worker forgetting than it was in the LFCM.

\subsection{The interactions between $F A P$ and $N$}

\subsection{LFCM}

Given an upfront training policy of $F A P-2$, varying $N$ has no significant effect on performance. However, given a $F A P$ level of 0 or 1, decreasing $N$ from three to two causes a significant increase in performance. Irrespective of the number of tasks learned, increasing the $F A P$ level from 0 to 1 provides significant benefits; however, a further increase to $F A P-2$ provides no significant additional benefit. Performance is best when the upfront training policy 
is $F A P-1$ and the number of tasks learned is two. Performance is poorest when there is no upfront training $(F A P-0)$ and the number of tasks learned is three; however, an exception is when the worker transfer policy is 250 and/or the forgetting rate is low (95\%), in these special cases forgetting losses are significantly reduced.

\subsection{DPLFM}

The interactions of the DPLFM differ from the previous LFCM interactions by the following: at an upfront training policy of $F A P-1$, varying the level of $N$ has no significant effect on performance, whereas in the LFCM at $F A P-1$, decreasing $N$ from three to two provided significant benefits. Also, the special 3-way interactions are identical for the upfront training policy and the number of tasks learned in both models.

\subsection{Discussion}

Again, it is evident, that the relative influences of some factors in the LFCM cause interaction differences between models. Namely, in this interaction, the performance is slightly less affected by the level of the number of tasks learned factor $(N)$ in the DPLFM than in the LFCM. This is probably because $N$ is also less influential in the DPLFM than in the LFCM (see Figure $4.2(\mathrm{c}))$.

\subsection{Summary of the interaction effect (LFCM and DPLFM)}

It is apparent from the previous comparison of the 2-way and 3-way interaction analysis between the DPLFM and the LFCM that the factors $C, F A P, F R$, and $N$ are less of an influence in the DPLFM than in the LFCM (this concurs with the main effect conclusions see Figure 4.2(c)). This was evident because of the following: (1) the insensitivity of the level of the worker forgetting rate and worker transfer policy was greater in the DPLFM than in the LFCM in the $C / F R$ interaction, (2) the insensitivity of the level of the worker forgetting rate was also greater in the DPLFM than in the LFCM in the $F R / N$ interaction, (3) the insensitivity of the level of the number of tasks learned factor $N$ was greater in the DPLFM than in the LFCM in the $F A P / N$ interaction, (4) the detrimental affect on forgetting losses of no upfront training $(F A P-0)$ and a small transfer policy $(C=10)$ was unresolved by a reduction in worker forgetting to $95 \%$ in the LFCM in the C/FAP interaction, whereas, in the DPLFM, the 
forgetting reduction was sufficient in mitigating forgetting losses; this highlights the greater influence of both factors in the LFCM model, and, (5) in the $C / N$ interaction, only an upfront training policy of $F A P-2$ is significant in reducing the harshest case of forgetting losses in the DPLFM, whereas in the LFCM, just FAP-1 is sufficient. This suggests the overwhelming influence of the upfront training policy in the LFCM as compared with the DPLFM.

The inclusion of the task related factors inherent in the DPLFM model such as task-type $(T)$ and the ratio of time for the first unit under purely cognitive conditions to time for the first unit under purely motor conditions $(R)$ were probably responsible for the change in the relative main effect of the factors illustrated in the main effect conclusions (see Figure 4.2(c)) and the above comparison of the interaction effect of the LFCM and the DPLFM.

\subsubsection{Discussion of the interaction effects unique to the DPLFM}

\subsection{The interactions between $C$ and $T$}

The performance increases as the worker transfer policy and the degree of cognitive elements in the task increase. However, given a worker transfer level of 250 , varying the task-type has no significant effect on performance. Given a worker transfer policy of 25 , changing from a tasktype $T_{\text {Med }}$ to $T_{U}$, from $T_{C}$ to $T_{U}$, or from $T_{\text {Med }}$ to $T_{C}$, has no significant affect on performance. Given a worker transfer policy of 10 , changing from a task-type $T_{M e d}$ to $T_{U /}$, or from $T_{U}$ to $T_{C}$ does not significantly affect performance, whereas any other task-type change causes a significant change. Also, given a worker transfer policy of 10 or 25 , changing the task-

type from $T_{M}$ to any other task significantly benefits performance. Given task-type $T_{M}$ and $T_{M e d}$, increasing the worker transfer policy at any level significantly improves performance. Also, given task-type $T_{C}$ or $T_{U}$, increasing the worker transfer policy from 10 to 25 significantly improves performance, however, an additional increase to 250 provides no further benefits. Performance is best when the worker transfer policy is 250 and the task-type is $T_{C}$. Performance is poorest when the worker transfer policy is 10 and the task-type is $T_{M}$; however, an exception is when the upfront training is $F A P-2$ or $F A P-1$, and/or the worker 
forgetting rate is low (95\%), in these special cases forgetting losses are significantly reduced.

\subsection{Discussion}

At this interaction, it appears that the beneficial effect of a worker transfer policy of 250 overrides any affect of task-type on performance. It is interesting to note that, except during an infrequent worker transfer policy $(C=250)$, changing the task-type from $T_{M}$ to any other task significantly benefits performance. This finding suggests that it may be almost always beneficial to change the task-type if it is predominantly a motor task. Also, it is interesting to note that, performing the uniform task $T_{U}$ reduces forgetting losses more than $T_{\text {Med }}$, even though the learning rate of $T_{U}$ is equal to the average of task $T_{\text {Med }}$. These results concur with the main effect findings that forgetting losses may be underestimated if the dual cognitive and motor learning elements are not taken into account. It also appears that providing any upfront training $(F A P-1$ or $F A P-2)$ or reducing worker forgetting can significantly counteract a harsh situation of performing a task $T_{M}$ with a frequent worker transfer of 10 .

\subsection{The interactions between $C$ and $R$}

Given a worker transfer policy of 250 , changing the $R$-value of the task has no significant effect on shop performance. Given a worker transfer policy of 10, increasing the $R$-value of the task at any level yields significant performance benefits. However, given a worker transfer policy of 25, changing the $R$-value of the task from one to three, or from one-third to one, does not yield a significant change in performance, whereas a change from one-third to three does provide a significant improvement. Given that the $R$-value of the task is one-third or one, increasing the worker transfer policy at any level significantly improves performance. Also, given that the $R$-value of the task is three, increasing the worker transfer policy from 10 to 25 significantly improves performance; however, a further increase to 250 provides no further significant benefits. Performance is best when the worker transfer policy is 250 and the $R$-value of the task is three. Performance is poorest when the worker transfer policy is 10 and the $R$ value of the task is one-third; however, an exception is when the forgetting rate is low (95\%), and/or upfront training is given ( $F A P-1$ or $F A P-2)$, in these special cases forgetting losses are significantly reduced. 


\subsection{Discussion}

Again, it is evident that an increased worker transfer policy is sufficient in reducing forgetting losses irrespective of the qualities of the task, in this case the $R$-value of the task. In fact, when $C=250$, varying the task-type does not affect shop performance at this interaction. Also, irrespective of worker transfer policy level, the relative influence of varying the $R$-value of the task was small within the range chosen. This concurs with the main effect results, see Figure 4.1(c) comparing $C$ versus $R$ in the DPLFM. Also, since three special 3-way cases could alleviate the harsh conditions of $C_{10} R_{1 / 3}$, this 2-way condition may not be a crucial issue.

\subsection{The interactions between $F R$ and $T$}

Given a situation of high forgetting ( $85 \%$ ), changing the task-type to any setting, except for a task-type change from $T_{\text {Med }}$ to $T_{U}$, or from $T_{U}$ to $T_{C}$, has a significant effect on shop performance. Given a medium or low worker forgetting rate $(90 \%$ or $95 \%)$, only changing the task-type from $T_{M}$ to any other task significantly improves performance; any other task-type change does not significantly affect performance. At every task-type except $T_{C}$, any decrease in worker forgetting at any level improves shop performance. Given the worker performs a cognitive task, reducing the worker forgetting from $85 \%$ to $90 \%$ significantly improves performance; however, an additional decrease to a worker forgetting rate of $95 \%$ provides no further significant benefits. Interestingly, irrespective of worker forgetting rate, performing a uniform task $\left(T_{U}\right)$ does not significantly improve performance over a cognitive task $\left(T_{C}\right)$. Again, it is interesting to note that performing task $T_{11}$, a task with no specified motor or cognitive elements, is more beneficial in reducing forgetting losses than performing task $T_{\text {Med }}$, a task that has both learning elements but has an averaged combined learning rate equal to that of $T_{U}$. Performance is best when the forgetting rate is low (95\%) and the task-type is $T_{C}$. Performance is poorest when the forgetting rate is high (85\%) and the task-type is $T_{M}$; however, an exception is when the worker transfer policy is 250 and/or upfront training is given ( $F A P-1$ or $F A P-2$ ), in these special cases forgetting losses are significantly reduced.

\subsection{Discussion}

The results of the above interaction suggest that significant benefits can be achieved by changing 
the task-type to counteract the forgetting losses associated when a worker with a high forgetting rate performs a more motor dominated task, especially when the positive effects of a worker transfer policy of 250 and/or upfront training are not available (3-way interaction exceptions). Also, in this interaction, the underestimation of forgetting losses when the dual learning elements are not recognized is evident when $T_{U}$ is performed versus $T_{\text {Med }}$. As in the main effect conclusions (see Table 4.3), the benefits provided from changing from a $T_{U}$ task to a $T_{C}$ task are minimal, especially in medium and low forgetting rates. Again, the detrimental effect of performing motor dominated tasks is evident because changing from a $T_{M}$ task to any other task is beneficial for two out of three worker forgetting rate levels. Also, at this particular interaction, with the exception of performing a cognitive task, decreasing the forgetting rate at any level provides significant performance improvements.

\subsection{The interactions between $F R$ and $R$}

Given a low forgetting rate (95\%), varying the $R$-value at any level has no significant effect on performance. Given a medium forgetting rate (90\%), significant benefits may result if the $R$ value is increased from one-third to three; however an $R$-value change from one-third to one, or from one to three is not sufficient in yielding significant benefits. Given a high worker forgetting rate (85\%), increasing the $R$-value of the task at any level provides significant performance improvements. Given that the $R$-value of the task is one-third or one, decreasing the forgetting rate at any level provides significant benefits. However, given that the $R$-value of the task is three, decreasing the worker forgetting from $85 \%$ to $90 \%$ provides significant benefits, whereas an additional decrease to $95 \%$ provides no further significant benefits. Performance is best when the forgetting rate is low (95\%) and the $R$-value of the task is three. Performance is poorest when the forgetting rate is high (85\%) and the $R$-value of the task is one-third; however, an exception is when there is upfront training ( FAP-1 or FAP-2), and/or the worker transfer policy is 250 ; in these special cases forgetting losses are significantly reduced.

\subsection{Discussion}

The result of the above interaction suggest that the $R$-value of the task has a less overall affect on performance as compared with the forgetting rate. This is evident because, given that the $R$ value is one-third or one (two out of three levels), reducing the forgetting rate greatly enhances performance, whereas, for example, the influence of the $R$-value is only of interest at a high forgetting rate (one out of three levels). Nonetheless, an $R$-value of three provides enough 
additional benefit that a further decrease in worker forgetting from $90 \%$ to $85 \%$ provides no significant benefits $(-4.09 \%)$. Also, the special case 3-way interactions of upfront training $(F A P-1$ or $F A P-2$ ), and/or a worker transfer policy of 250 provide a significant reduction of forgetting losses in the harshest 2-way interaction $F R_{85 \%} R_{1 / 3}$.

\subsection{The interactions between $T$ and $F A P$}

Given any task-type, increasing the upfront training policy beyond $F A P-1$ provides no significant additional benefit. Given an upfront training policy of $F A P-1$ or $F A P-2$, a task-type change at any level does not significantly affect performance. However, given no upfront training $(F A P-0)$, any change in task-type, except from a $T_{M e d}$ to a $T_{U}$ task, significantly affects performance. Performance is best when the task-type is $T_{C}$ and the upfront training policy is $F A P-2$. Performance is poorest when the task-type is $T_{M}$ and there is no upfront training $(F A P-0)$; however, an exception is when the worker transfer policy is 250 and/or the worker forgetting rate is low (95\%) in these special cases forgetting losses are significantly reduced.

\subsection{Discussion}

In this interaction, the results suggest that the upfront training policy factor $(F A P)$ is much more influential on performance than the task-type factor, this finding concurs with the main effect results, see Figure 4.1(c). For example, given upfront training levels of $F A P-1$ or $F A P-2$, performance is insensitive to the change in task-type, whereas, conversely, given any task-type, providing just the standard time number of units $(F A P-1)$ of upfront training is effective in significantly reducing forgetting losses. In this interaction, the special case 3-way interaction of a worker transfer policy of 250 and/or a low worker forgetting rate (95\%) reduces the harshest of forgetting losses.

\subsection{The interactions between $T$ and $N$}

The results suggest that, given that a worker performs a task $T_{C}$, the number of tasks learned has no significant effect on performance. However, given any other task $\left(T_{M}, T_{\text {Med }}\right.$, or $\left.T_{U}\right)$, decreasing the number of tasks learned from three to two causes a significant increase in 
performance. Given that the number of tasks learned is two, changing from a task $T_{M}$ to $T_{C}$, or from $T_{M}$ to $T_{U}$ causes a significant increase in performance; however, any other task-type change has no significant effect on performance. Given that the number of tasks learned is three, changing from a motor task $\left(T_{M}\right)$ to any other task causes a significant increase in performance; however, any other task-type change has no significant effect on performance. Performance is best when the task-type is $T_{C}$ and the number of tasks learned is two. Performance is poorest when the task-type is $T_{M}$ and the number of tasks learned is three; however, an exception is when the worker transfer policy is $250 \mathrm{and} /$ or the worker forgetting rate is low $(95 \%)$ and/or the upfront training policy is $F A P-2$, in these special cases forgetting losses are significantly reduced.

\subsection{Discussion}

In this interaction, the results suggest that the performance of a cognitive task provides enough forgetting losses to deem the number of tasks learned factor immaterial. Also, similarly, the results suggest that if the number of tasks learned is three, only a task-type change from a motor task to any other task causes a significant increase in performance. Therefore, since the factor of task-type has one level $\left(T_{C}\right)$ that nullifies the significance of the effect of varying the levels of the other factor $N$, and the number of tasks learned factor has one level $(N=3)$ that nullifies the significance of the effect of varying the levels of the other factor $T$ (with the exception of changing from a task $T_{M}$ ), the factors $N$ and $T$ appear nearly equal with respect to their contributions to performance. This concurs with the main affect results; see Figure 4.1(c). Again, the special case 3-way interactions of: a worker transfer policy of 250 , and/or a low worker forgetting rate (95\%), and/or the upfront training policy is $F A P-2$, significantly reduces the harshest factor 2-way interaction $T_{M} N_{3}$.

\subsection{The interactions between $T$ and $R$}

Given task-type $T_{U}$, varying the $R$-value of the task has no significant effect on performance.

Given task-type $T_{M}$, increasing the $R$-value at any level yields a significant increase in performance. Given task $T_{\text {Med }}$, increasing the $R$-value from one-third to one provides significant benefits, however, an additional increase to an $R$-value of three provides no further 
significant benefits. Given task $T_{C}$, the performance is only significantly increased if the $R$ value is increased from one-third to three; no significant benefit result from just an increase from one-third to one, or from one to three. Also, as expected, $T_{U}$ is not significantly affected by the change in $R$-value since there are no distinct cognitive or motor elements in the task. Given a task with an $R$-value of one-third, every task-type change, except from $T_{U}$ to $T_{C}$, causes a significant change in performance. Given a task with an $R$-value of one, only a task-type change from $T_{M}$ to $T_{C}$, or from $T_{M}$ to $T_{U}$ causes a significant change, every other task-type change has no significant effect on performance. However, given a task with an $R$-value of three, the task-type factor has no significant effect on performance. Performance is best when the task-type is $T_{C}$ and the $R$-value of the task is three. Performance is poorest when the task-type is $T_{M}$ and the $R$-value of the task is one-third; however, an exception is when there is upfront training provided ( $F A P-1$ _or $F A P-2$ ), and/or the worker transfer policy is 250 , in these special cases forgetting losses are significantly reduced.

\subsection{Discussion}

An interesting observation is the relative lack of performance sensitivity with the change in tasktype when the $R$-value of the task is three. Also, it is evident how an increasing fraction of motor elements increases the relative benefit of changing from task $T_{\text {Med }}$ to $T_{U}$ or from $T_{\text {Med }}$ to $T_{C}$; this is because the detrimental effect of performing a task containing a motor element is exacerbated as the fraction of motor content increases. This interaction is important because it stresses that even though the $R$-value factor has, overall, the second smallest main effect, it interacts closely with the task-type factor (a higher ranked main effect factor), and as a result, its importance should not be entirely overlooked.

\subsection{The interactions between $F A P$ and $R$}

Given no upfront training $(F A P-0)$, increasing the $R$-value of the task at any level significantly increases performance. However, given upfront training ( FAP-1 or $F A P-2$ ), changing the $R$-value of the task has no significant effect on performance. Irrespective of the $R$-value of the task, increasing the upfront training policy from $F A P-0$ to $F A P-1$ yields increased performance; however, a further increase to $F A P-2$ provides no significant additional benefit. Performance is best when the upfront training policy is $F A P-2$ and the 
$R$-value of the task is three. Performance is poorest when there is no upfront training $(F A P-0)$ and the $R$-value of the task is one-third; however, an exception is when the worker transfer policy is 250 and/or the worker forgetting rate is low (95\%), in these special cases forgetting losses are significantly reduced.

\subsection{Discussion}

In this interaction, there are effects that, again, have a strong influence on performance, such as: the benefit of just providing $F A P-1$, and the minor influence of the $R$-value of a task in the presence of upfront training. Changing the $R$-value of a task at any level is indeed only significant when there is no upfront training $(F A P-0)$, whereas just the addition of $1 \times n_{s}$ amount of upfront training causes the $R$-value of the task factor to be immaterial. This finding concurs with the main effect results (see, Figure 4.1(c)) that the upfront training factor (FAP) overshadows the effect of the $R$-value factor.

\subsection{The interactions between $N$ and $R$}

Irrespective of the number of tasks learned, the performance is only significantly increased if the $R$-value of the task is increased from one-third to three; no significant benefits result from just an increase from an $R$-value of one-third to one, or from one to three. Given that the $R$-value of the task is one-third or one, decreasing the number of tasks learned from three to two significantly improves performance. However, given that the $R$-value of the task is three, the number of tasks learned factor has no significant effect on performance. Performance is best when the number of tasks learned is two and the $R$-value of the task is three. Performance is poorest when the number of tasks learned is three and the $R$-value of the task is one-third; however, an exception is when the upfront training policy is $F A P-2$ and/or the worker transfer policy is 250 and/or the forgetting rate is low (95\%), in these special cases forgetting losses are significantly reduced.

\subsection{Discussion}

In this interaction, the observations reiterate the minor effect of the $R$-value factor. However, given that an $R$-value of three causes the number of tasks learned factor to be immaterial, the significance of the $R$-value factor should not be entirely overlooked. In addition, this interaction reflects the main effect conclusion that only learning two tasks is preferable. 


\subsubsection{Interaction effect conclusions - DPLFM}

Several notable conclusions are inferred from the previous analysis. Firstly, in the DPLFM, the worker transfer level of 250 and upfront training (especially $F A P-2$ ) remain important policies for reducing the harshest 2-way forgetting losses, as they were included in 10 $(C=250)$, nine $(F A P-2)$, and six $(F A P-1)$ out of the 34 special case 3-way upper interaction exceptions (located below the upper interaction criterion, see inequality in (4.4)). However, in eight of the interactions, upfront training and/or a reduced transfer frequency were not necessary for reducing the harshest 2-way forgetting losses if the worker forgetting rate was low (95\%). Also, it may also be of interest to note that performing a task-type $T_{U /}$ came within $0.5 \%$ of exceeding the upper interaction criterion of the 2-way interaction $F A P-0-R_{1 / 3}$. Again, this highlights the significance of how much the forgetting losses may be underestimated by not accounting for the dual phase cognitive-motor elements of a task. There were no observed 3-way interaction exceptions for a best case 2-way interaction (located above the lower interaction criterion, see inequality in (4.4)).

Secondly, a remarkable observation was that changing the task-type from $T_{M}$ to any other task significantly reduces forgetting losses in three out of five 2-way interactions containing the tasktype factor $(C / T, F R / T, T / N)$. Forgetting losses are significantly reduced in these cases except for when the worker transfer policy is 250 in the $C / T$ interaction, when the worker forgetting rate is low (95\%) in the $F R / T$ interaction, and when the number of tasks learned is two in the $T / N$ interaction. This finding provides credit to the superb benefit of trying to avoid the production of a task-type $T_{M}$; however, this is unnecessary in the above specified 2way interactions if the forgetting rate of the worker is low (95\%), if an employer can reduce the worker transfers to every 250 units, or if the number of tasks learned is kept at two.

Thirdly, the interaction effect of the $R$-value factor of the task was found to be inferior to the other factors; this concurred with the main effect conclusions. However, the $R$-value factor still may be of significance because the results suggest that even though the $R$-value factor has, overall, the second smallest main effect, it interacts closely with the task-type factor (a higher ranked main effect factor). Also, a task that has three times more motor than cognitive elements ( $R_{1 / 3}$ ) came within $1.1 \%$ of exceeding the lower interaction criterion, and as a result, nearly caused the only exception for a best case 2-way interaction $\left(C_{250} F R_{95 \%}\right)$. Furthermore, in the 
interaction $N / R$, a task with an $R$-value of three caused the number of tasks learned factor to be immaterial. Therefore, because of the above reasons, the significance of the $R$-value factor should not be entirely overlooked.

Finally, as in the LFCM, there is no additional benefit for the worker to learn three tasks instead of just two tasks. However, the results of the interaction effect suggest that the level of the number of tasks learned factor $(N)$ is insignificant if: the worker transfer policy is 250 in the $C / N$ interaction, the worker forgetting rate is low (95\%) in the $F R / N$ interaction, the upfront training policy is $F A P-2$ in the $F A P / N$ interaction, the task being performed is cognitive $\left(T_{C}\right)$ in the $T / N$ interaction, or the $R$-value of the task is three in the $N / R$ interaction.

\subsubsection{Comparison of the results of model A with Jaber et al. (2003)}

Jaber et al. (2003) conducted their experiment in order to answer the following three questions: (A) Will providing a worker upfront training reduce forgetting? (B) How does the frequency of worker transfers $(C)$ relate to forgetting? $(C)$ Do the answers to the above questions $\mathrm{A}$ and $\mathrm{B}$ change as the number of tasks for which a worker is trained increases or for different forgetting rates? The interplay between the upfront training policy $(F A P)$ and the worker transfer policy $(C)$ in the LFCM was also examined. In this section, the results of Jaber et al. (2003) are compared with the results of modeling worker learning and forgetting using the DPLFM and are discussed as they relate to the aforementioned A, B, C questions and (D): the interplay between upfront training and transfer policy.

\subsubsection{Discussion of question (A)}

Jaber et al. (2003) concluded that, in the LFCM, increasing worker training from $F A P-0$ to $F A P-1$ to $F A P-2$ reduced forgetting losses. The percentage improvement from $F A P-0$ to $F A P-2$ was $29.4 \%$ for the LFCM (see Table 4.2 ). It can also be concluded that, when using the DPLFM, increasing worker training from $F A P-0$ to $F A P-1$ to $F A P-2$ also reduces forgetting losses. Upfront training in the DPLFM reduces forgetting 
losses less than in the LFCM; this is evident since the overall relative improvement from $F A P-0$ to $F A P-2$ in the DPLFM was $17.2 \%$ (see Table 4.3 ).

\subsubsection{Discussion of question (B)}

Jaber et al. (2003) concluded that, in the LFCM, reducing the frequency of worker transfers (increasing the worker transfer policy from 10 to 25 to 250) reduces forgetting losses. The percentage benefit by changing the worker transfer policy from 10 to 250 is $24.1 \%$ for the LFCM (see Table 4.2). It can also be concluded that, when using the DPLFM, reducing the frequency of worker transfers reduces forgetting losses. However, the magnitude of forgetting loss reduction provided by reducing the frequency of worker transfers in the DPLFM from 10 to 250 is less (14.5\%) than that of the LFCM (see Table 4.3).

\subsubsection{Discussion of question (C)}

Due to the complexity of the interacting experimental factors, the conclusions made by Jaber et al. (2003) regarding question $C$ are numbered and then referenced by the corresponding conclusions of model A. Also, the conclusions for the DPLFM in this section were acquired by examining the DPLFM results of model A in a suppressed format (similar to the results of Jaber et al. (2003)), see appendix section 7.5.

Conclusion (1) of Jaber et al. (2003) suggested that, in the LFCM, given a worker transfer policy of $10, F A P-1$ was all that was needed to reduce forgetting losses at all forgetting rates when the number of tasks learned was two, and (conclusion (2)) with a low forgetting rate when the number of tasks learned is three. Conclusion (3) suggested that, when three tasks are learned, and the forgetting rate is medium or high, there is additional benefit to providing more upfront training to $F A P-2$. Conclusion (4) suggested that, given no upfront training ( $F A P-0$ ), increasing the worker transfer policy from 10 to 25 is sufficient to counter forgetting losses for two tasks learned and a low forgetting rate. In conclusion (5) it was suggested that, given no upfront training $(F A P-0)$, for medium and high forgetting rates, and for three tasks learned, there is an additional benefit to increasing worker transfer policy to 250 .

The results of this experiment suggest the following: conclusion (1) of Jaber et al. (2003) is true in the DPLFM with the exception of when a worker with a high forgetting rate (85\%) performs a task $T_{M}$ with an $R$-value of one-third ( $\left.F R_{85 \%} T_{M} R_{1 / 3}\right)$; in this case even upfront training to 
two times the standard number of units $(F A P-2)$ is ineffective in significantly reducing forgetting losses. However, conclusion (2) is true in the DPLFM at all task-types and $R$-values. Conclusion (3) only concurs with the following DPLFM factor settings of $C_{10} F R_{90 \%} T_{\text {Med }} R_{1 / 3}, C_{10} F R_{85 \%} T_{C} R_{1}, C_{10} F R_{85 \%} T_{\text {Med }} R_{3}, C_{10} F R_{85 \%} T_{U} R_{\mathrm{1} / 3}$, $C_{10} F R_{85 \%} T_{U} R_{3}, C_{25} F R_{85 \%} T_{C} R_{1 / 3}, C_{25} F R_{85 \%} T_{C} R_{1}, C_{25} F R_{85 \%} T_{M} R_{3}, C_{25} F R_{85 \%} T_{M e d} R_{1}$, $C_{25} F R_{85 \%} T_{U} R_{1 / 3}, C_{25} F R_{85 \%} T_{U} R_{1}$, and $C_{25} F R_{85 \%} T_{U} R_{3}$, at all other settings either $F A P-2$ is insufficient in providing forgetting loss reductions, $F A P-1$ is all that is required to reduce forgetting losses, or even no upfront training is required at all $(F A P-0)$ to significantly reduce forgetting losses. Namely, only 12 out of the possible 72 factor settings specified within the criteria of conclusion (3) concurred with the LFCM. As a result, it is evident how influential the task-type and $R$-value factors are at certain settings of $N$ and $F R$. Overall, conclusion (4) differs from the results of the LFCM because, given the settings of conclusion (4) of FAP-0,N=2, and $F R=95 \%$, the shop conditions in the DPLFM already provide significant forgetting loss reductions regardless of the level of the worker transfer policy. Also, interestingly, for the factor setting of $N_{2} F R_{95 \%} T_{M} R_{1 / 3}$, increasing the worker transfer policy is actually insufficient in significantly reducing forgetting losses, a further increase to $C=250$ is required due to the inherent magnitude of the motor elements of the task at this setting. Conclusion (5) is also true in the DPLFM with the exception of when a worker with a medium forgetting $(90 \%)$ rate performs a task $T_{C}$ with an $R$-value of one or three, or a task $T_{M e d}$ with an $R$-value of three $\left(F R_{90 \%} T_{C} R_{1}, F R_{90 \%} T_{C} R_{3}, F R_{90 \%} T_{M e d} R_{3}\right)$. In these cases, the amount of forgetting losses is so small initially that only an increase to a worker transfer policy of 25 is necessary to reduce forgetting losses. Also, conversely, even an increase to a worker transfer policy of $250(C=250)$ in conclusion (5) is insufficient in reducing forgetting losses in the DPLFM when a worker with a medium forgetting rate $(90 \%)$ performs a task $T_{M}$ with an $R$-value of one-third ( $\left.F R_{90 \%} T_{M} R_{1 / 3}\right)$, when a worker with a high forgetting rate $(85 \%)$ performs a task $T_{M}$ with an $R$-value of one-third or one $\left(F R_{85 \%} T_{M} R_{1 / 3}\right.$ or $F R_{85 \%} T_{M} R_{1}$ ), or when a worker with a high forgetting rate (85\%) performs a task $T_{\text {Med }}$ with an $R$-value of one-third ( $F R_{85 \%} T_{M e d} R_{1 / 3}$ ). 


\subsubsection{Discussion of issue (D)}

The conclusions made by Jaber et al. (2003) regarding issue D are numbered and then referenced by the corresponding conclusions of model A. Conclusion (1) of Jaber et al. (2003) suggested that, given two tasks learned, a low forgetting rate (95\%), a transfer policy of 10 , and no initial training ( $F A P-0$ ), either increasing the transfer policy (to 25 or 250 ) or introducing initial training (to $F A P-1$ or $F A P-2$ ) would significantly remove forgetting losses. It is not necessary to both increase the transfer policy and provide upfront training, as either method alone is sufficient in reducing forgetting losses. Conclusion (2) stated that the general pattern in conclusion (1) holds as the forgetting rate increases to medium $(90 \%)$ or high $(85 \%)$ and/or the number of tasks learned increases to three. Conclusion (3) suggested that, given that three tasks are learned and a high forgetting rate, both providing upfront training and increasing the transfer policy are required to achieve maximum forgetting loss reduction.

Conclusion (1) of Jaber et al. (2003) is also true in the DPLFM. In fact, only the factor setting of $N_{2} F R_{95 \%} T_{M} R_{1 / 3}$ requires the use of either upfront training or an increased transfer policy to reduce forgetting, all other factor settings within those specified in conclusion (1) are already significantly absent of forgetting losses even before any policy changes. Conclusion (2) is true in the DPLFM, with the exception of when a worker with a high forgetting rate learns two tasks of type $T_{M}$ with an $R$-value of one-third ( $\left.N_{2} F R_{85 \%} T_{M} R_{\mathrm{I} / 3}\right)$, when a worker with a medium forgetting rate learns three tasks of type $T_{M}$ with an $R$-value of one-third ( $N_{3} F R_{90 \%} T_{M} R_{1 / 3}$ ), when a worker with a high forgetting rate learns three tasks of type $T_{M}$ with an $R$-value of one-third or one $\left(N_{3} F R_{85 \%} T_{M} R_{1 / 3}, N_{3} F R_{85 \%} T_{M} R_{1}\right)$, or when a worker with a high forgetting rate learns three tasks of type $T_{M e d}$ with an $R$-value of one-third $\left(N_{3} F R_{85 \%} T_{M e d} R_{1 / 3}\right)$, in these cases increasing the worker transfer policy and introducing initial training are not even sufficient in removing forgetting losses. Again, conclusion (3) is true in the DPLFM with the exception of when a task $T_{\text {Med }}$ with an $R$-value of one-third $\left(T_{M c d} R_{1 / 3}\right)$ is performed, or when a task $T_{M}$ with an $R$-value of one-third or one $\left(T_{M} R_{1 / 3}\right.$ or $\left.T_{M} R_{1}\right)$ is performed, in these cases increasing the worker transfer policy and introducing initial training are not even sufficient in removing forgetting losses. 
It is interesting to note that all three issue (D) DPLFM conclusions suggested that the detrimental effect of performing a motor dominated task is such that the combined effort of providing upfront training and increasing the transfer policy may be insufficient in countering forgetting losses.

\subsubsection{Implications of results for the five DRC issues}

The five possible issues that affect DRC shop performance measures as given by Hottenstein and Bowman (1998) are: (1) worker flexibility, (2) centralization of control, (3) worker assignment, (4) queue disciplines, and, (5) cost of transferring workers. The results of model A are compared to these issues and are summarized briefly below.

\subsubsection{Issue 1: worker flexibility (number of tasks learned)}

Hottenstein and Bowman (1998) found that cross-training beyond two or three skills per worker does not significantly enhance DRC system performance. In model A, and in Jaber et al. (2003), it was evident that, overall (see row two, column four of Table 4.3), training workers beyond two skills does not benefit shop performance; and as a result, the results in model A concur with those of Jaber et al. (2003) and Hottenstein and Bowman (1998).

\subsubsection{Issue 2: centralization of control ('when' rule)}

The survey by Hottenstein and Bowman (1998) regarding centralization of control concluded the following: (1) an efficiency control rule that moves a worker as soon as the worker can be moved to a stage that he/she is most efficient is shown to be a superior rule under most conditions; (2) the degree of centralized control is not independent of the assignment rule; and, (3) centralized control only marginally reduces mean and variance of flow-time compared to decentralized control; and if the efficiency levels of the workers at various tasks differ, then the level-of-control-decision is far less dependent on the status of the queue but is determined by the time of the availability of a station where the worker is more efficient.

In model A, and in Jaber et al. (2003), the implications of the above conclusions are as follows. Regarding conclusion (1), in model $\mathrm{A}$, it was assumed that a worker is equally efficient in all stages; therefore this analysis is not applicable. As for conclusion (2), the degree of independence of the centralized control was not addressed in either model A or in Jaber et al. 
(2003) because the worker was always assigned to the next available job and different assignment rules were not examined. Regarding conclusion (3) in model A, and in Jaber et al. (2003), the $\pi_{i}$ (the level of centralized control of jobs in stage $i$ parameter), is neither 0 nor 1 but varies between these two values. More specifically, the level of centralized control is determined by the number of units processed. The worker moves from the station after a fixed number of jobs are completed $(C)$, e.g.: batch sizes of 10,25 , or 250 . However, the conclusion (3) by Hottenstein and Bowman (1998) suggests that a level of control closer to 0 than to 1 is preferred. In model A (see row 13, column two in Table 4.3), and in Jaber et al. (2003), out of the possible levels of 10,25 and 250 , it was found that a worker transfer policy of 250 reduced forgetting losses most significantly. As a result, it can be inferred that the results of model $A$ and those of Jaber et al. (2003) differed from those researched by Hottenstein and Bowman (1998) because they concluded that a centralized control policy was superior to a decentralized policy; albeit, Hottenstein and Bowman (1998) concluded that it was superior by only a marginal amount. Again, as for the remaining remarks in conclusion (3), the analysis in model A and in Jaber et al. (2003) is not applicable because the worker is assumed equal efficiency on all stations.

\subsubsection{Issue 3: worker assignment}

As previously mentioned, since the worker in model A and in Jaber et al. (2003) was always assigned to the next available job, different assignment rules were not examined.

\subsubsection{Issue 4: queue disciplines}

There are no arrivals in model A or in Jaber et al. (2003) until a unit is complete, therefore, it is a simple static, first-come-first-serve model. As a result, the comparison between different queue discipline rules was not made.

\subsubsection{Issue 5: cost of transferring workers}

There are no conclusions made in either model A or in Jaber et al. (2003) because no transfer delay was assumed and hence the costs of transferring workers was assumed zero. 


\subsubsection{Model A conclusions}

The purpose of the experiment conducted in model A was to determine if modeling worker learning and forgetting according to the dual-phase learning-forgetting model (DPFLM) changed the conclusions made by Jaber et al. (2003), where worker learning and forgetting was modeled according to the LFCM. Determining if there was a difference may be important because the DPLFM is the only learning and forgetting model that closely models the seven characteristics of learning as summarized by Jaber et al. (2003), and theoretically models cognitive and motor learning elements that were validated experimentally; and as a result, model A may provide more appropriate conclusions regarding the effectiveness of DRC performance enhancing policies. The implications of the results of model $A$ on the five possible issues that affect DRC shop performance given by Hottenstein and Bowman (1998) were also addressed.

The following conclusions can be made. Model A suggested that the DPLFM credits less benefit for an increased upfront training policy $(F A P)$ and a less frequent worker transfer policy $(C)$ than the LFCM (see Table 4.3 versus Table 4.2). The difference of benefit for $F A P$ and $C$ in the DPLFM is due to the additional consideration for the task-type that is being performed. This difference is evident in the discussions in sections 4.1.3.3 and 4.1.3.4 when a highly motor dominated task is being performed; especially when the $R$-value of the task is low. The results of model A suggest that the nature of the task being performed with respect to its learning rate $(T)$ and proportion of cognitive and motor elements $(R)$ may be an important issue, and therefore, could be brought under consideration for future DRC research. The DPLFM is a model that can provide a measure that recognizes both these inherent task characteristics. In fact, the results of model A suggest that the task-type factor $(T)$ is of such importance that the detrimental effect of performing a motor dominated task $\left(T_{M}\right)$ is such that the combined effort of providing upfront training and increasing the transfer policy may be insufficient in countering forgetting losses in workers with a high forgetting rate $(F R=85 \%)$ when two tasks are learned, and in workers with a medium ( $F R=90 \%$ ) and high ( $F R=85 \%$ ) forgetting rate when three tasks are learned.

It was also observed that upfront training was still an important factor in reducing the forgetting losses in the DPLFM; albeit at a slightly less degree than in the LFCM. In Jaber et al. (2003), it was suggested that since the results of the LFCM showed that upfront training was more important in the LFCM than in the VRVF, the LFCM more closely modeled tasks that were more dissimilar. The results of model A suggest that the DPLFM stresses the importance of upfront training more than the VRVF but less than the LFCM. As suggested by Jaber et al. 
(2003), what the worker does during an interruption period may affect the degree of forgetting losses. As a result, the inclusion of the similarity factor in model B of this thesis examines how the task similarity factor interacts with the DPLFM as compared with the LFCM. In addition, it was observed that further upfront training to $F A P-2$ was more beneficial in the DPLFM than in the LFCM. An interesting observation of model A was that performing task $T_{U}$ resulted in less forgetting losses than performing $T_{\text {Med }}$, a task with an equal average learning rate. Also, it was of interest to note that performing a task-type $T_{U}$ came within $0.5 \%$ of exceeding the upper interaction criterion of the 2-way interaction $F A P-0-R_{1 / 3}$. As a result, it can then be inferred that forgetting losses may be underestimated when the dual cognitive and motor task elements are not distinguished from each other as they are in the DPLFM.

Model A suggested that learning an additional task (from $N=2$ to $N=3$ ) increases forgetting losses; however, the detrimental effect of learning an additional task was slightly less in the DPLFM than in the LFCM. Also, the number of tasks learned factor $(N)$ is insignificant in specific 2-way interactions if the worker transfer policy is 250 , if the worker forgetting rate is low (95\%), if the upfront training policy is $F A P-2$, or if the task being performed is cognitive. Also, the interaction effect analysis of model A illustrated how some factor settings reduce the influence of the levels of less significant factors. For example, given upfront training levels of $F A P-1$ or $F A P-2$ in the 2-way interaction $T / F A P$, performance is insensitive to the change in task-type, whereas, conversely, given any task-type, providing just one-times the standard time number of units $(F A P-1)$ of upfront training is sufficient to significantly reduce forgetting losses. This observation stresses the importance of the magnitude of interaction between factors and how this interaction may affect conclusions drawn by only considering the factors individually (as in the main effect results).

As for the unique DPLFM factor interactions, it was found that, the interaction effect of the $R$ value of the task was found to be inferior to the other factors. However, the $R$-value factor still may be of significance because the results suggest that even though the $R$-value factor has, overall, the second smallest main effect, it closely interacts closely with the task-type factor (a higher ranked main effect factor). Also, a task that has three times more motor than cognitive elements $\left(R_{1 / 3}\right)$ came within $1.1 \%$ of exceeding the lower interaction criterion, and as a result, nearly caused the only exception for a best case 2-way interaction $\left(C_{250} F R_{95 \%}\right)$. Furthermore, in the interaction $N / R$, a task with an $R$-value of three caused the number of tasks learned 
factor to be immaterial. Therefore, because of the above reasons, the significance of the $R$ value factor should not be entirely overlooked.

The avoidance of producing a task of type $T_{M}$ is suggested by the results of model A; however, this is unnecessary in specified 2-way interactions if the forgetting rate of the worker is low (95\%), if an employer can reduce the worker transfers to every 250 units, or if the number of tasks learned is kept at two. Finally, in the DPLFM, the worker transfer level of 250 and upfront training (especially $F A P-2$ ) remain important policies for reducing the harshest 2-way forgetting losses, as they were included in $10(C=250)$, nine $(F A P-2)$, and six ( $F A P-1)$ out of the 34 special case 3-way upper interaction exceptions for reducing the harshest 2-way forgetting losses.

\subsection{Model B results}

Appendix section 7.6 contains the tables for the suppressed ${ }^{* * *}$ average processing time results for the DPLFM when two tasks $(N=2)$ and three tasks $(N=3)$ are learned with various degrees of similarity for the three worker transfer levels $(C=10, C=25, C=250)$.

Jaber et al. (2003) examined how the inclusion of the task similarity factor affected the results of the original assumption of no task similarity. The following section examines how the results of model B compare to those of Jaber et al. (2003), where the LFCM was used.

\subsubsection{Comparison of the results of model B with Jaber et al. (2003)}

Intuitively, what a worker does during an interruption period may affect the amount of forgetting that occurs. Jaber et al. (2003) suggested that a worker forgets less if he/she performs tasks that are similar during the interruption period. The original assumptions of the LFCM and the DPLFM were that the tasks performed are totally dissimilar; and hence, do not contribute to experience gained during an interruption. Jaber et al. (2003) concluded that the results of the

\footnotetext{
$\$ * *$

The theoretical average processing time is 1.04 . For ease of interpretation, the results from 1.04 and 1.06 have been suppressed with a "-". This is consistent with the formatting of results of Jaber et al. (2003).
} 
LFCM with the similarity factor for two tasks learned suggested that the benefit or motivation to provide upfront training or a reduced transfer frequency decreases with increased similarity because the average processing times decrease as similarity increases. The model $B$ results tables in appendix section 7.6.1 suggest that this is also true for the DPLFM, irrespective of the new factors of task-type ( $T$ ) and the $R$-value of the task. It can be concluded that the DPLFM also attributes less forgetting losses with increased levels of task similarity; this concurs with the findings of Jaber et al. (2003).

However, for two tasks learned, the relative degree of forgetting loss reduction is less pronounced in the DPLFM than in the LFCM. This is evident when the change in the average processing times between task similarity levels is averaged irrespective of all other factors in each model, see Table 4.6. As shown in the shaded cells of Table 4.6, the overall reduction in average processing time from a task similarity level of $s=0$ to $s=0.8$ is less in the DPLFM than in the LFCM. It can then be inferred that forgetting loss reduction in the DPLFM is less

Table 4.6: Percent decrease in average processing times with increasing task similarity $^{* * *}$ - the DPLFM versus the $\operatorname{LFCM}(N=2)$

\begin{tabular}{|l|l|l|}
\hline Change in the degree of & DPLFM & LFCM \\
task similarity $(\Delta s)$ & & \\
\hline $0.0-0.2$ & $-1.39 \%$ & $-1.91 \%$ \\
\hline $0.0-0.4$ & $-2.75 \%$ & $-3.93 \%$ \\
\hline $0.0-0.6$ & $-3.96 \%$ & $-6.20 \%$ \\
\hline $0.0-0.8$ & $-5.07 \%$ & $-8.78 \%$ \\
\hline
\end{tabular}

The baseline for comparison is no task similarity, it is calculated as follows: $[\{A P T(s=i)-A P T(s=0)\} / A P T(s=0)]^{*} 100$, where $i=0.0,0.2,0.4,0.6$ 
sensitive to changes in the degree of task similarity; and hence, there is slightly more motivation to reduce forgetting losses when performing similar tasks with upfront training or a reduced transfer frequency in the DPLFM than in the LFCM. Again, for the suppressed average processing time results in the DPLFM when three tasks are learned with various degrees of task similarity (see appendix section 7.6.2), it is evident that forgetting losses reduction is significant as the task similarity level increases.

The conclusions made by Jaber et al. (2003) regarding the effect of the similarity factor on the LFCM when three tasks are learned are numbered as follows: (1) Jaber et al. (2003) concluded that, for the LFCM when three tasks are learned, when task similarity is low and forgetting rates are high, extensive training $(F A P-2)$ alone cannot completely reduce forgetting losses, and reducing the frequency of worker transfers is necessary to reduce forgetting losses; and (2) the results of Jaber et al. (2003) suggested, however, that as the degree of task similarity increases ( $S_{2}$ or greater), even a moderate amount of training $(F A P-1)$ is sufficient in reducing forgetting losses without also having to resort to more frequent worker transfers.

Conclusion (1) of Jaber et al. (2003) is true with the exception of when a cognitive task $\left(T_{C}\right)$ with an $R$-value of one is performed, when a median task ( $T_{\text {Med }}$ ) with an $R$-value of three is performed, or when a uniform task ${ }^{\dagger \dagger \dagger}$ with an $R$-value of one-third or three is performed (bold font numbers in $85 \%$ forgetting rate column of the suppressed average processing time results for the DPLFM with the task similarity factor $-C=10, N=3$, see Table 4.7); in these cases extensive training $(F A P-2)$ is sufficient in reducing forgetting losses without having to reduce the frequency of worker transfers. In addition, when a cognitive task $\left(T_{C}\right)$ with an $R$ value of three is performed (bold and italicized font), just moderate training $(F A P-1)$ is sufficient in reducing forgetting losses. Conclusion (2) of Jaber et al. (2003) is true with the DPLFM with the following exceptions: (a) when a cognitive task $\left(T_{C}\right)$ with an $R$-value of three is performed, in this case, tasks can be totally dissimilar (similarity factor of $S_{0}$ or greater) in order for moderate training $(F A P-1)$ to be sufficient in reducing forgetting losses, (b) when a cognitive task with an $R$-value of one, or when a median task ( $\left.T_{M e d}\right)$ with an $R$-value of three is performed, in these cases tasks can be even less similar (similarity factor of $S_{1}$ or greater) in order for $F A P-1$ to be sufficient in reducing forgetting losses,

${ }^{t+t}$ As in model A, forgetting losses may be underestimated if the cognitive and motor task requirements are not accounted for. 
Table 4.7: Suppressed average processing time results for the DPLFM with the task similarity factor $-C=10, N=3$

\begin{tabular}{|c|c|c|c|c|c|c|c|c|c|c|c|}
\hline \multirow[b]{2}{*}{$\begin{array}{l}\text { Task- } \\
\text { Type }\end{array}$} & \multirow[b]{2}{*}{$R$} & \multirow[b]{2}{*}{$\begin{array}{l}\text { Similarity } \\
\text { Factor } \\
(S)\end{array}$} & \multicolumn{3}{|c|}{$\begin{array}{l}\text { Low forgetting } \\
\text { rate of } 95 \%\end{array}$} & \multicolumn{3}{|c|}{$\begin{array}{l}\text { Medium forgetting } \\
\text { rate of } 90 \%\end{array}$} & \multicolumn{3}{|c|}{$\begin{array}{l}\text { High forgetting } \\
\text { rate of } 85 \%\end{array}$} \\
\hline & & & $F A P-0$ & $F A P-1$ & $F A P-2$ & $F A P-0$ & $F A P-1$ & $F A P-2$ & $F A P-0$ & $F A P-1$ & $F A P-2$ \\
\hline \multirow{12}{*}{$T_{C}$} & \multirow{4}{*}{$1 / 3$} & $S_{0}$ & 1.1263 & - & - & 1.7420 & - & - & 2.0664 & 1.2708 & 1.1274 \\
\hline & & $\mathbf{S}_{\mathbf{t}}$ & - & - & - & 1.6165 & - & - & 1.9494 & 1.1365 & - \\
\hline & & $\mathbf{S}_{2}$ & - & - & - & 1.4097 & - & - & 1.7521 & - & - \\
\hline & & $\mathbf{S}_{3}$ & - & - & - & - & - & - & 1.2537 & - & - \\
\hline & \multirow{4}{*}{1} & $S_{0}$ & - & - & - & 1.4647 & - & - & 1.8559 & 1.1903 & - \\
\hline & & $\mathbf{S}_{1}$ & - & - & - & 1.3355 & - & - & 1.7331 & - & - \\
\hline & & $S_{2}$ & - & - & - & 1.1110 & - & - & 1.5284 & - & - \\
\hline & & $\mathbf{S}_{\mathbf{3}}$ & $=$ & - & - & - & - & - & - & - & . \\
\hline & \multirow{4}{*}{3} & $\mathrm{~S}_{\mathbf{0}}$ & - & - & - & 1.0833 & - & - & 1.4711 & - & - \\
\hline & & $\mathbf{S}_{1}$ & - & - & - & - & - & - & 1.3361 & - & - \\
\hline & & $\mathbf{S}_{\mathbf{2}}$ & - & - & - & - & - & - & 1.0946 & - & - \\
\hline & & $S_{3}$ & - & - & - & - & - & - & - & - & - \\
\hline \multirow{12}{*}{$T_{M}$} & \multirow{4}{*}{$1 / 3$} & $S_{0}$ & $\begin{array}{l}.7488 \\
\end{array}$ & - & - & 2.3971 & 1.1351 & 1.1079 & 2.6494 & 1.4259 & 1.3012 \\
\hline & & $\mathbf{S}_{\mathbf{1}}$ & 1.6160 & - & - & 2.2934 & & - & 2.5583 & 1.3008 & 1.2062 \\
\hline & & $\mathbf{S}_{\mathbf{2}}$ & 1.4007 & - & - & 2.1180 & - & - & 2.3982 & 1.1180 & 1.0936 \\
\hline & & $S_{3}$ & 1.0733 & - & - & 1.6663 & - & - & 1.9671 & - & - \\
\hline & \multirow{4}{*}{1} & $\mathrm{~S}_{\mathbf{0}}$ & 1.3887 & - & - & 2.0008 & - & - & 2.3408 & 1.3416 & 1.2275 \\
\hline & & $S_{1}$ & 1.2530 & - & - & 1.8835 & - & - & 2.2349 & 1.2105 & 1.1083 \\
\hline & & $\mathbf{S}_{\mathbf{2}}$ & - & - & - & 1.6888 & - & - & 2.0543 & - & - \\
\hline & & $S_{3}$ & - & - & - & 1.2186 & - & - & 1.5878 & - & - \\
\hline & \multirow{4}{*}{3} & $\mathbf{S}_{0}$ & - & - & - & 1.6476 & - & - & 1.9818 & 1.2181 & 1.0805 \\
\hline & & $\mathbf{S}_{\mathbf{1}}$ & - & - & - & 1.5207 & - & - & 1.8627 & 1.0833 & - \\
\hline & & $\mathbf{S}_{\mathbf{2}}$ & - & - & - & 1.3132 & - & - & 1.6637 & - & - \\
\hline & & $S_{3}$ & - & - & - & & - & $=$ & 1.1724 & - & - \\
\hline \multirow{12}{*}{$T_{M e d}$} & \multirow{4}{*}{$1 / 3$} & $\mathrm{~S}_{0}$ & 1.4156 & - & - & 2.0616 & 1.0919 & - & 2.3549 & 1.3814 & 1.2599 \\
\hline & & $S_{1}$ & 1.2772 & - & - & 1.9451 & - & - & 2.2491 & 1.2475 & 1.1343 \\
\hline & & $\mathbf{S}_{\mathbf{2}}$ & - & - & - & 1.7505 & - & - & 2.0676 & - & - \\
\hline & & $\mathbf{S}_{3}$ & - & - & - & 1.2689 & - & - & 1.5943 & - & - \\
\hline & \multirow{4}{*}{1} & $\mathrm{~S}_{\boldsymbol{0}}$ & 1.0811 & - & - & 1.7204 & - & - & 2.0906 & 1.2697 & 1.1301 \\
\hline & & $\mathbf{S}_{\mathbf{I}}$ & - & - & - & 1.5957 & - & - & 1.9751 & 1.1379 & - \\
\hline & & $\mathbf{S}_{2}$ & - & - & - & 1.3911 & - & - & 1.7803 & - & - \\
\hline & & $S_{3}$ & - & - & - & - & - & - & 1.2895 & - & - \\
\hline & \multirow{4}{*}{3} & $\overline{\mathbf{S}_{0}}$ & $=$ & - & - & 1.3773 & - & - & 1.7287 & 1.1005 & - \\
\hline & & $\mathbf{S}_{1}$ & - & - & - & 1.2432 & - & - & 1.6030 & - & - \\
\hline & & $S_{2}$ & - & - & - & - & - & - & 1.3956 & . & - \\
\hline & & $\mathbf{S}_{\mathbf{3}}$ & - & - & - & - & - & - & - & - & - \\
\hline & & $\mathbf{S}_{0}$ & - & - & - & 1.6480 & - & - & 1.9866 & 1.2271 & - \\
\hline & $1 / 3$ & $S_{1}$ & - & - & - & 1.5175 & - & - & 1.8654 & 1.0824 & - \\
\hline & $1 / 3$ & $\mathbf{S}_{\mathbf{2}}$ & - & - & - & 1.3025 & - & - & 1.6615 & - & - \\
\hline & & $S_{3}$ & - & - & - & - & - & - & 1.1477 & - & - \\
\hline & & $\mathbf{S}_{\mathbf{0}}$ & 1.0850 & - & $=$ & 1.7472 & - & - & 2.1198 & 1.2937 & 1.1291 \\
\hline$T$ & 1 & $\mathbf{S}_{1}$ & - & - & - & 1.6194 & - & - & 2.0034 & 1.1559 & - \\
\hline${ }^{I} U$ & 1 & $\mathbf{S}_{\mathbf{2}}$ & - & - & - & 1.4075 & - & - & 1.8045 & - & - \\
\hline & & $\mathbf{S}_{\mathbf{3}}$ & - & - & - & - & - & - & 1.2905 & - & - \\
\hline & & $\mathbf{S}_{\mathbf{0}}$ & - & - & - & 1.6480 & - & - & 1.9866 & 1.2271 & - \\
\hline & 3 & $\mathbf{S}_{\mathbf{I}}$ & - & - & - & 1.5175 & - & - & 1.8654 & 1.0824 & - \\
\hline & 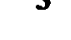 & $\mathbf{S}_{\mathbf{2}}$ & - & - & - & 1.3025 & - & - & 1.6615 & - & - \\
\hline & & $\mathrm{S}_{\mathbf{3}}$ & - & - & - & - & - & $\therefore$ & 1.1477 & - & - \\
\hline
\end{tabular}


and, (c) when a motor task $\left(T_{M}\right)$ with an $R$-value of one-third is performed, in this case tasks need to be almost totally similar (similarity factor of $S_{3}$ or greater) in order for moderate training $(F A P-1)$ to be sufficient in reducing forgetting losses. The aforementioned exceptions to conclusion (2) are illustrated in Table 4.7 by shaded numbers.

Also, for three tasks learned, the degree of forgetting loss reduction is less pronounced in the DPLFM than in the LFCM. This is evident when the change in the average processing times between task similarity levels is averaged irrespective of all other factors in each model, see Table 4.8. As shown in shaded cells of Table 4.8, the overall reduction in average processing time from a task similarity level of $S_{0}$ to $S_{3}$ is less in the DPLFM than in the LFCM. It can then be inferred that forgetting loss reduction in the DPLFM is also less sensitive to changes in the degree of task similarity when three tasks are learned; and hence, there is slightly more motivation to reduce forgetting losses when performing similar tasks with upfront training or a reduced transfer frequency in the DPLFM than in the LFCM. These findings also support the deduction made in the main effect results of model A that the DPLFM accrues less importance on the dissimilarity of the tasks being performed than the LFCM. Also, Jaber et al. (2003) concluded that it is possible to use greater flexibility in shops with similar tasks. The results of model B suggest that this is even truer for the DPLFM (see shaded values of the DPLFM versus the LFCM column in Table 4.9). For example, Jaber et al. (2003) concluded that the assumption of the LFCM with no task similarity suggested that an increase to $N=3$ provided no further benefit.

The overall average processing time in the LFCM when two tasks are learned $(N=2)$ (irrespective of all factors) is 1.16 (see bold font number in the $N=2, s=0$ cell of Table 4.9). The equivalent value for the DPLFM is 1.07 (also in bold font). It can then be inferred that a combination of factors yielding an average value equal to or less than 1.16 for the LFCM, or 1.07 for the DPLFM, may suggest an equally feasible policy. As shown in the shaded $N=3$ values for the LFCM, tasks should have a similarity of at least $S_{3}$ or greater in the LFCM for $N=3$ to be as feasible as $N=2$ with no similarity. However, in the DPLFM, only a task similarity of $S_{2}$ or greater is necessary for the feasibility of training workers for three tasks. Therefore, the results of model $B$ indicate that it may be even more feasible than suggested by Jaber et al. (2003) that it is possible to use greater flexibility in shops with similar tasks. 
Table 4.8: Percent decrease in average processing times with increasing task similarity ${ }^{\ddagger \neq}$ - the DPLFM versus the $\operatorname{LFCM}(N=3)$

\begin{tabular}{|l|r|l|}
\hline $\begin{array}{l}\text { Change in the degree of } \\
\text { task similarity }\end{array}$ & DPLFM & LFCM \\
\hline$S_{0}-S_{1}$ & $-2.83 \%$ & $-4.37 \%$ \\
\hline$S_{0}-S_{2}$ & $-6.00 \%$ & $-9.58 \%$ \\
\hline$S_{0}-S_{3}$ & $-9.97 \%$ & $-16.43 \%$ \\
\hline
\end{tabular}

Table 4.9: Average processing times with respect to number of tasks learned and task similarity factor - the DPLFM and the LFCM

\begin{tabular}{|c|c|c|c|c|c|}
\hline \multicolumn{3}{|c|}{ DPLFM } & \multicolumn{3}{|c|}{ LFCM } \\
\hline \multirow{5}{*}{$N=2$} & 0.0 & 1.07 & \multirow{5}{*}{$N=2$} & 0.0 & 1.16 \\
\hline & 0.2 & 1.06 & & 0.2 & 1.14 \\
\hline & 0.4 & 1.04 & & 0.4 & 1.12 \\
\hline & 0.6 & 1.03 & & 0.6 & 1.09 \\
\hline & 0.8 & 1.02 & & 0.8 & 1.06 \\
\hline \multirow{4}{*}{$N=3$} & $S_{0}$ & 1.14 & \multirow{4}{*}{$N=3$} & $S_{0}$ & 1.29 \\
\hline & $S_{I}$ & 1.11 & & $\overline{S_{I}}$ & 1.24 \\
\hline & $S_{2}$ & 1.07 & & $S_{2}$ & 1.17 \\
\hline & $S_{3}$ & 1.03 & & $S_{3}$ & 1.08 \\
\hline
\end{tabular}

\subsubsection{Implication of results for the five DRC issues}

The five possible issues that affect DRC shop performance measures as given by Hottenstein and Bowman (1998) are: (1) worker flexibility, (2) centralization of control, (3) worker assignment, (4) queue disciplines, and, (5) cost of transferring workers. The results of model B are compared to these issues and are summarized as follows.

\#\# The values in Table 4.8 are calculated as in Table 4.6 


\subsubsection{Issue 1: worker flexibility (number of tasks learned)}

Hottenstein and Bowman (1998) found that cross-training beyond two or three skills per worker does not significantly enhance DRC system performance. In model B, and in the similarity section of Jaber et al. (2003), it was evident that training workers for up to three skills may be possible without severe forgetting losses in cases where there is similarity between tasks. Furthermore, the results of model $B$ indicate that it may be even more feasible than suggested by Jaber et al. (2003) that it is possible to use greater flexibility in shops with similar tasks. As a result, the results in model B support those of Jaber et al, (2003) and Hottenstein and Bowman (1998).

\subsubsection{Issue 2: centralization of control ('when' rule)}

The survey by Hottenstein and Bowman (1998) regarding centralization of control concluded the following: (1) an efficiency control rule that moves a worker as soon as the worker can be moved to a stage that he/she is most efficient is shown to be a superior rule under most conditions, (2) the degree of centralized control is not independent of the assignment rule, and, (3) centralized control only marginally reduces mean and variance of flow-time compared to decentralized control; and if the efficiency levels of the workers at various tasks differ, then the level-of-control-decision is far less dependent on the status of the queue but is determined by the time of the availability of a station where the worker is more efficient. The implications of the results of model $\mathrm{B}$ regarding the above three issues are the same as in model $\mathrm{A}$.

\subsubsection{Issue 3: worker assignment}

As previously mentioned, since the worker modeled in model B and in Jaber et al. (2003) was always assigned to the to the next available job, different assignment rules were not examined

\subsubsection{Issue 4: queue disciplines}

There are no arrivals in model B or in Jaber et al. (2003) until a unit is complete; therefore, it is a simple static, first-come-first-serve model. As a result, the comparison between different queue discipline rules was not made. 


\subsubsection{Issue 5: cost of transferring workers}

There are no conclusions made in either model B or in Jaber et al. (2003) because no transfer delay was assumed and hence the costs of transferring workers was assumed zero.

\subsubsection{Model B conclusions}

It can then be concluded that when worker learning and forgetting is modeled according to the DPLFM, the effect of the task similarity factor is affected by the type of task that is being performed with regards to the effectiveness of upfront training, frequency of worker transfers, and the number of tasks learned. The main findings of model B are as follows: (1) the results suggest that the DPLFM may emphasize a greater benefit for upfront training and more a frequent worker transfer policy than the LFCM when tasks are similar, and (2) model B supported the conclusions of Jaber et al. (2003) by an even greater extent that it is possible to use more flexibility (up to three tasks learned) in DRC shops with similar tasks, and, (3) the findings support the deduction made in the main effect results of model $A$ that the DPLFM accrues less importance on the dissimilarity of the tasks being performed than the LFCM. Consequently, the results of model $B$ suggest that the task-type factor, as modeled by the DPLFM, could also be included in DRC research when task similarity factors are examined.

\subsection{Model C results}

\subsubsection{Preliminary main effect analysis of $C^{*}$ and $F A P^{*}$}

An optimization search routine was programmed in Excel-Visual Basic. This computer procedure searched for the optimal set of output variables $\left(F A P^{*}, C^{*}\right)$ for each corresponding combination of input variables $(s, \rho, L R, F R)$. This produced a 5 (degree of task similarity $(s)$ ) $\times 5$ (initial processing time to standard time ratio $(\rho)) \times 5$ (worker learning rate $(L R)) \times 5$ (worker forgetting rate $(F R))$ table of results yielding 625 optimized values of the 
upfront training policy $\left(F A P^{*}\right)$ and the centralization of control policy $\left(C^{*}\right)$. The tables of results are given in the appendix section 7.7. As previously mentioned, the search range of $F A P^{*}$ was $\{F A P-0-F A P-5\}$ in increments of $0.5 \times n_{s}$ and the search range of $C^{*}$ was $\{10-250$ units $\}$ in increments of one unit.

Initially, the main effect of each input variable $(s, \rho, L R, F R)$ on the output variables $\left(F A P^{*}, C^{*}\right)$ were calculated. As previously mentioned, the main effect can be defined as the change in response or output caused by the change in a level of the factor in question (Montgomery, 1997). In this analysis, the main effect of each input variable on the optimized level of $F A P$ and $C$ was found by averaging each optimized value of $F A P^{*}$ and $C^{*}$ irrespective of the other levels of the input variables. For example, the effect of the degree of similarity was calculated by averaging all of the $F A P^{*}$ and $C^{*}$ values at each level of $s(0.0,0.2,0.4,0.6,0.8)$ over all of the levels of $\rho(2,3,4,5,6), L R(70 \%, 75 \%, 80 \%, 85 \%, 90 \%)$, and $F R(95 \%, 92.5 \%, 90 \%, 87.5 \%, 85 \%)$. As a result, the effect of the degree of similarity on both $C^{*}$ and $F A P^{*}$ was found by plotting the main effect of $C^{*}$ and $F A P^{*}$ at each level of $s$. This was performed for each input variable. This was done to determine if there were any mathematical trends between the levels of each input variable and the resulting optimized output variable. This section examines the behaviour of the main effect of each input variable on each of the output variables, the justification for their apparent mathematical relationship, and the conclusions that can be drawn from this analysis.

The resulting main effect calculations resulted in a total of eight plots: four for each of the input variables versus each output variable $C^{*}$ and $F A P^{*}$.

\subsubsection{Main effect analysis of $C^{*}$}

The main effect results for $C^{*}$ are given in Table 4.10. The results in Table 4.10 are subsequently illustrated as scatter plots that are used for analysis and discussion.

\subsubsection{1 $C^{*}$ versus $s$}

The scatter plot of the main effect behaviour of $C^{*}$ with degree of task similarity $(s)$ is given in Figure 4.3. As shown in Figure 4.3, the average optimal batch size appears to decrease with 
Table 4.10: The main effect of each input variable versus level of $C^{*}$

\begin{tabular}{|c|c|c|}
\hline Input variable & Level & $C^{*}$ \\
\hline \multirow{5}{*}{ Degree of task similarity $(s)$} & 0.0 & 109.0 \\
\hline & 0.2 & 94.5 \\
\hline & 0.4 & 75.8 \\
\hline & 0.6 & 50.0 \\
\hline & 0.8 & 24.3 \\
\hline \multirow{5}{*}{$\begin{array}{l}\text { Initial processing time to standard } \\
\text { time ratio }(\rho)\end{array}$} & 2 & 137.0 \\
\hline & 3 & 100.7 \\
\hline & 4 & 33.5 \\
\hline & 5 & 28.6 \\
\hline & 6 & 53.9 \\
\hline \multirow{5}{*}{$\begin{array}{l}\text { Worker learning rate }(L R) \text {, where } \\
L R=100 \times 2^{-b}\end{array}$} & $70 \%$ & 87.0 \\
\hline & $75 \%$ & 67.9 \\
\hline & $80 \%$ & 54.2 \\
\hline & $85 \%$ & 96.0 \\
\hline & $90 \%$ & 48.6 \\
\hline \multirow{5}{*}{$\begin{array}{l}\text { Worker forgetting rate }(F R) \text {, } \\
\text { where } F R=100 \times 2^{f}\end{array}$} & $95 \%$ & 35.4 \\
\hline & $92.5 \%$ & 52.3 \\
\hline & $90 \%$ & 74.0 \\
\hline & $87.5 \%$ & 91.4 \\
\hline & $85 \%$ & 100.6 \\
\hline
\end{tabular}

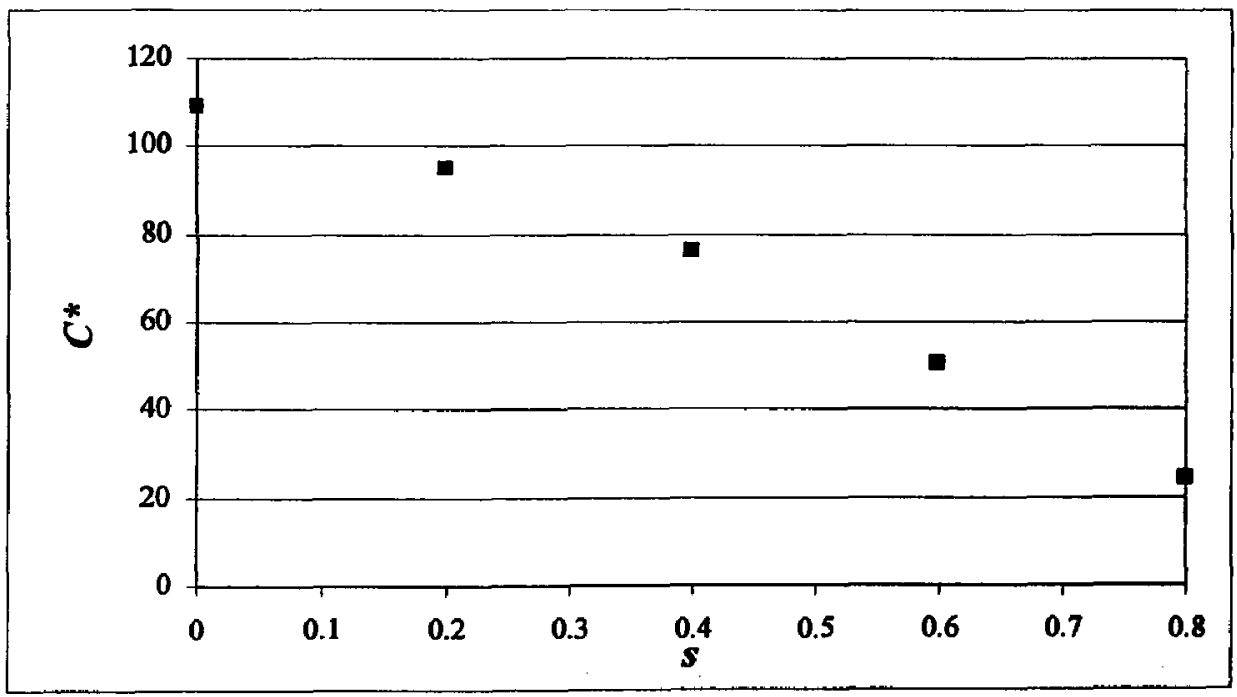

Figure 4.3: Plot of $C^{*}$ versus $s$ 
increasing levels of task similarity. This observation concurs with the results of Jaber et al. (2003) when it was noted that "..forgetting losses decrease with increasing similarity, there is less motivation to provide upfront training or reduce transfer frequency." Figure 4.3 also concurs with the mathematical relationship between the number of units remembered and the degree of task similarity. For example, the relationship between $s$ and the forget curve intercept is given by (Jaber et al., 2003):

$\hat{y}_{i}(1)=\left\{\begin{array}{l}(1-s) y(1)\left(u_{i}+n_{i}\right)^{-(b+f)}+s y(1)\left(u_{i}+n_{i}\right)^{-b}, \text { if }\left(u_{i}+n_{i}\right)<n_{s} \\ (1-s) y_{s}\left(u_{i}+n_{i}\right)^{-f}+s y_{s}, \text { otherwise }\end{array}\right.$

Simplifying equation (4.7) suggests a direct relationship:

$$
\hat{y}_{i}(1) \sim s,
$$

insomuch as when $s$ increases in the range $(0 \leq s \leq 1), \hat{y}_{i}(1)$ increases.

Therefore, in a similar manner, simplifying equations (2.2) and (2.7) give:

$$
\phi_{i} \sim \frac{1}{\hat{y}_{i}(1)}
$$

and

$$
u_{i} \sim \frac{1}{\phi_{i}}
$$

Consequently, substituting $S$ through equations (4.7a) to (4.8a) gives the following:

$$
u_{i} \sim s
$$

The relationship in (4.9) suggests that when the degree of task similarity increases $(0 \leq s \leq 1)$, the amount of experience retained at the beginning of cycle $i$ also increases, representing a decrease in forgetting losses between cycles. This also concurs with the relationship shown in Figure 4.3 , because, as the degree of task similarity increases, forgetting losses decrease, and therefore, there is less incentive to increase $C$. 


\subsubsection{2 $C^{*}$ versus $L R$}

The scatter plot of the main effect behaviour of $C^{*}$ versus learning rate is given in Figure 4.4.

Figure 4.4 suggests that $C^{*}$ tends to decrease as the learning rate slows; this is evident between learning rates of $70 \%$ to $80 \%$. This behaviour is contrary to intuition because it can be assumed that, as learning deteriorates, batch sizes should increase to compensate for the large forgetting losses. However, increasing $C$ greatly magnifies the detrimental effect of a slow learner. This is illustrated by plotting a graph of the average processing time ( $A P T$ ) versus the worker learning rate for several values of $C$ (no initial training: $F A P-0$ ), see Figure 4.5.

As shown in Figure 4.5, larger lot sizes (longer production cycles) become increasingly more effective in reducing $A P T$ values as learning slows. This observation is consistent with intuition but is also contradictory to the results depicted in Figure 4.4. However, it is important to reiterate that the data points in Figure 4.4 are the 'minimized' value of $C$ (or $C^{*}$ ) for all possible combinations of the factors previously mentioned in Table 3.5. As a result, averaging the values as in Figure 4.5 is beneficial for overall trends in forgetting losses but does not account for interactions between factors that have yielded optimized factor settings that only the search procedure in model $\mathrm{C}$ could have found. For example, factor interactions are overlooked by only examining one factor such as 'worker learning rate'. As previously mentioned, the interaction effect is when the change in response (output) between the levels of one factor is not the same at all the levels of the other factors (Montgomery, 1997). Montgomery (1997) clearly outlines this caveat of interactions when analyzing factors by using a simple example of a golfer experimenting with factors to improve his golf score, see Figure 4.6. As shown in Figure 4.6, the effect of the type-of-beverage-consumed factor (W-water versus B-beer) has little effect on the golf score when the regular-sized driver factor is used. However, at the other driver factor setting of 'oversized driver', the beverage factor is significant. Using this analogy in model C, this interaction effect is evident when the level of initial training moves from $F A P-0$ to $F A P-1$. Figure 4.7 illustrates the unique changes in the average processing time as initial training is introduced. Figure 4.7 reveals that, with some initial training ( FAP -1 ), and as learning slows, the minimized value of $C$ changes from $C^{*}=300$ when learning is $70 \%$ to $C^{*}=10$ when learning is $80 \%$. 


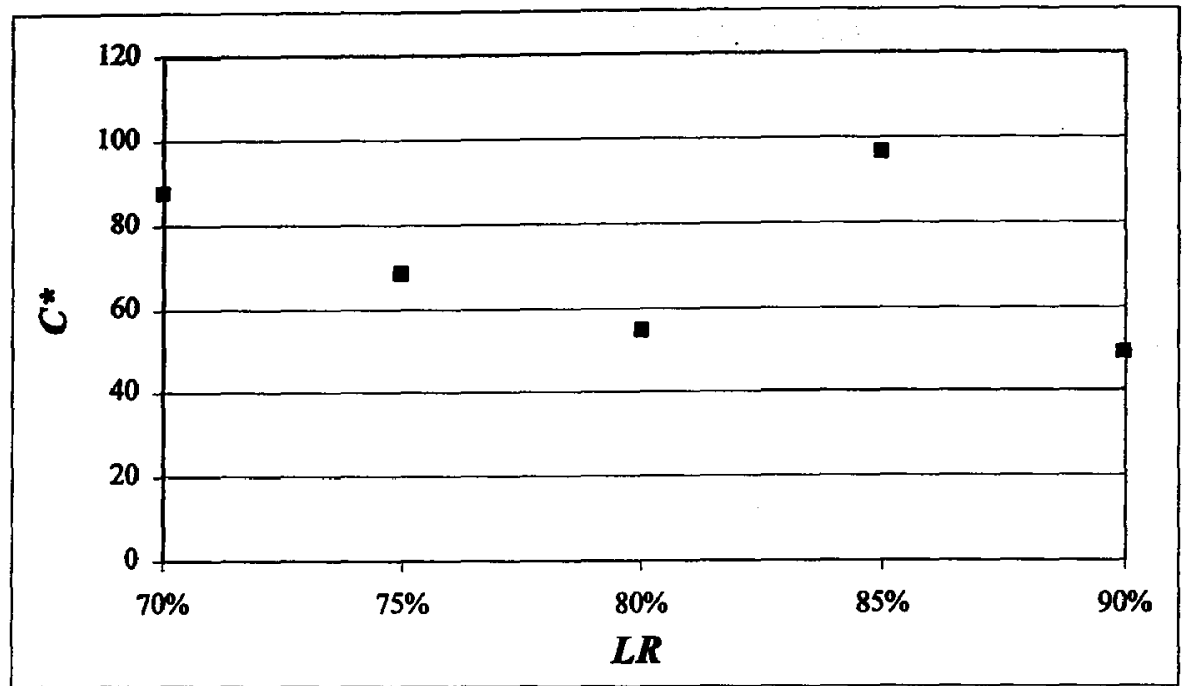

Figure 4.4: Plot of $C^{*}$ versus $L R$

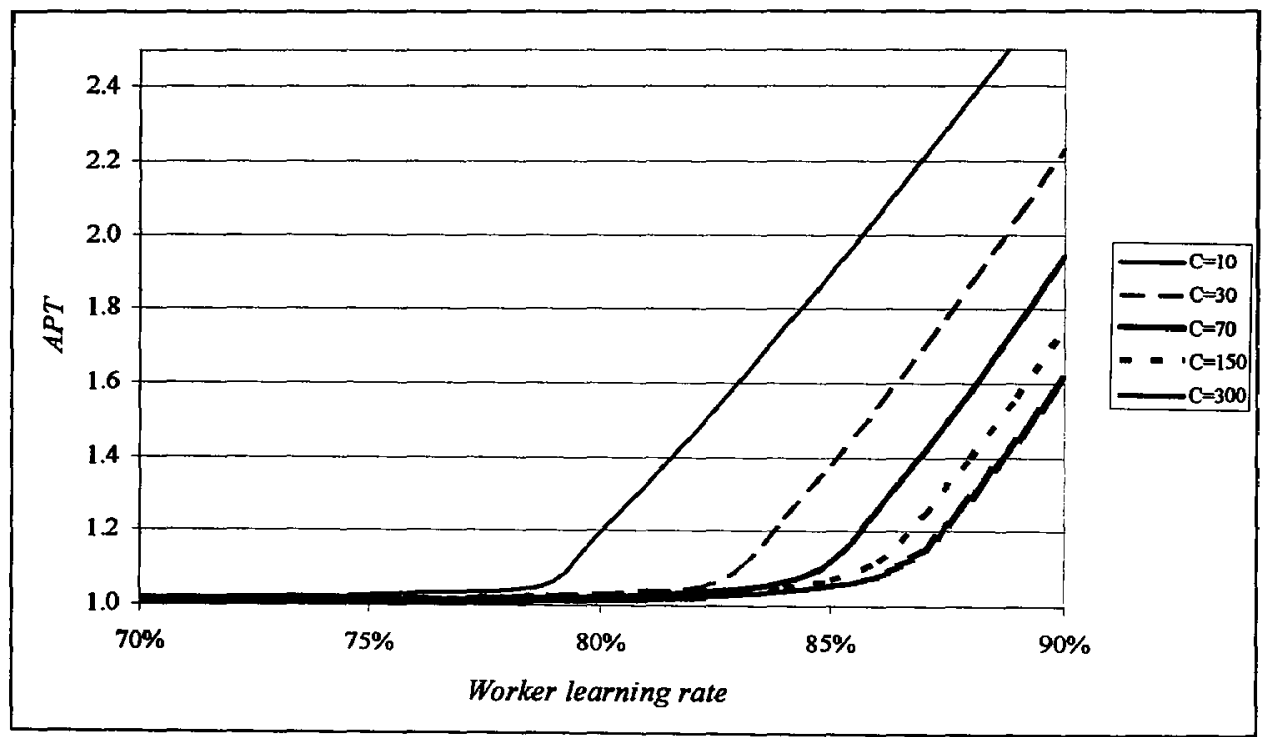

Figure 4.5: Average processing time ( $A P T)$ versus learning rate $(F A P-0)$ for several values of $C(s=0, N=2, F R=0.9, \rho=4)$ 


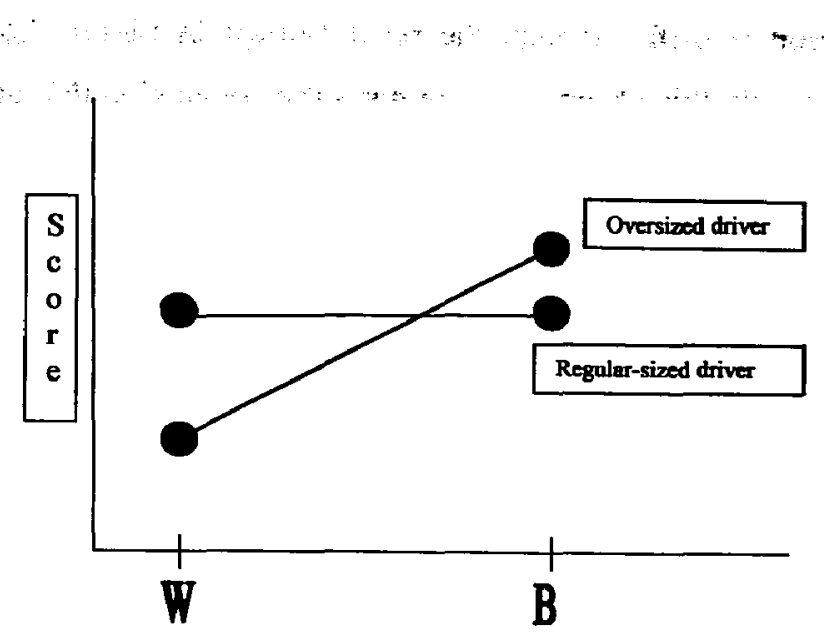

Figure 4.6: Interaction between type of driver and type of beverage for the golf experiment (Montgomery, 1997)

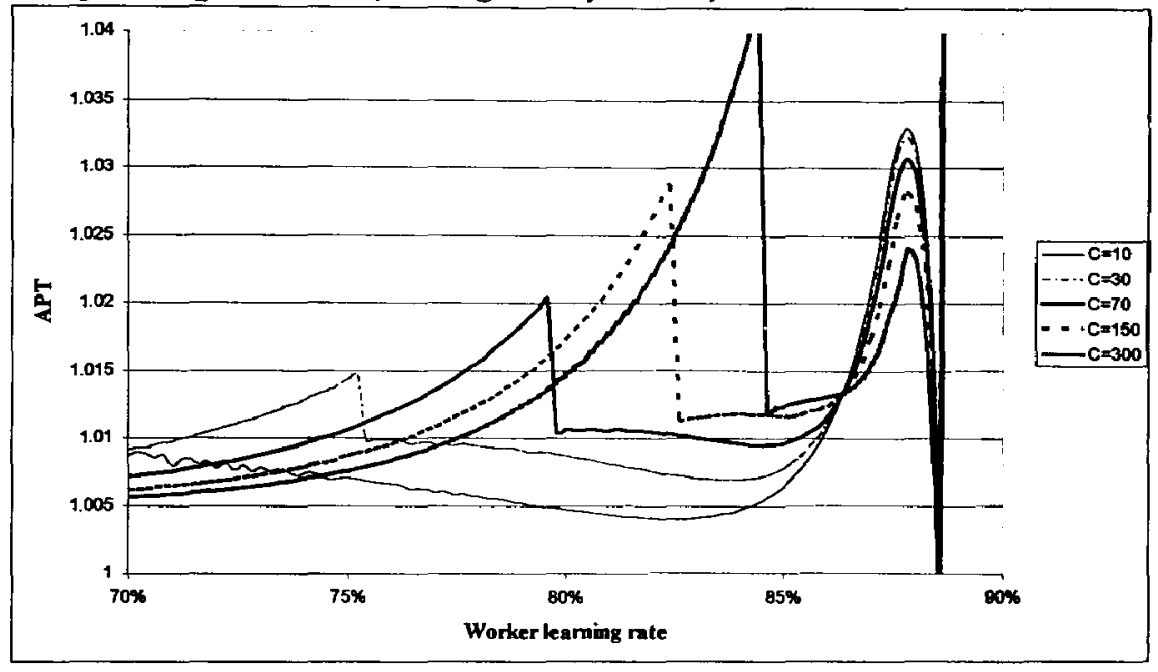

Figure 4.7: Average processing time $(A P T)$ versus learning rate $(F A P-1)$ for several values of $C(s=0, N=2, \rho=4)$

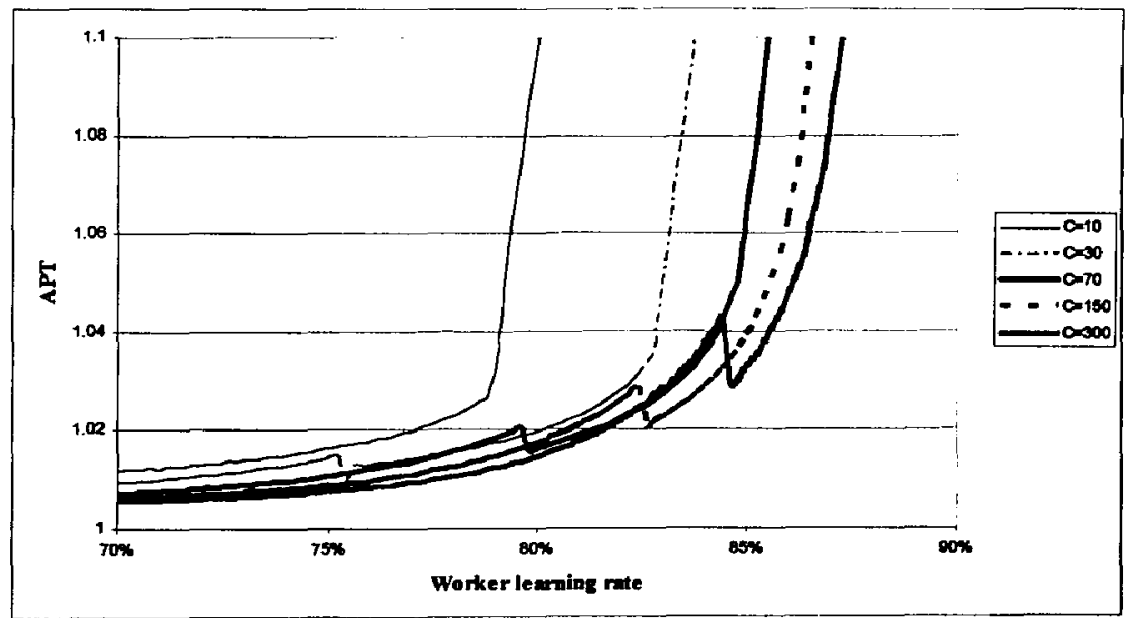

Figure 4.8: Average processing time $(A P T)$ versus learning rate for several values of $C$ averaged across $F A P-0$ and $F A P-1$ $(s=0, N=2, \rho=4)$ 
This observation is different than the result shown in Figure 4.5 where there was no initial training. As a result, this observation supports the conclusion that unique factor interactions may result in optimized performance measures that cannot be readily observed by just examining the performance behaviour at one factor setting. Also, unique factor interactions that yield minimized points cannot be found by averaging across factors such as in Figure 4.8, where the average processing time ( $A P T$ ) for both $F A P-0$ and $F A P-1$ were averaged.

Consequently, the results of Figure 4.4 are minimized values of $C\left(C^{*}\right)$ that are found in the search routine of model $\mathrm{C}$. This search routine included all the possible interactions of all the factors, and therefore, do not necessarily reflect overall trends concerning average processing times ( $A P T$ ) versus worker learning rate. This is true for all of the following main effect analyses of the optimized centralization of control policy $\left(C^{*}\right)$ and the optimized upfront training policy $\left(F A P^{*}\right)$. Having said this, however, some trends in the optimized values of $F A P$ and $C$ can be explained by examining the mathematical trends of the main factors. For example, Figure 4.4 also depicts $C^{*}$ increasing to a maximum at a learning rate of approximately $85 \%$ before decreasing again at a learning rate of $90 \%$. In order to further investigate the reasons for this behaviour, a 3-D plot of the time to total forgetting $(D)$ as a function of the worker learning rate and the initial specified forgetting rate was constructed as shown in Figure 4.9. Where the time to total forgetting $(D)$ is related to the transfer size and the learning and forgetting rate by:

$$
D(b, f, C)=\left[10^{\frac{b(1-b) \log (C)}{f}}-1\right] \times A P T_{1}
$$

and $A P T_{1}$ is the actual processing time in the first production cycle and is given by:

$$
A P T_{1}=\frac{y(1)}{1-b} C^{1-b}
$$

In all the models of this thesis, the time for total forgetting $(D)$ is calculated in the first production cycle and remains constant throughout all subsequent cycles. As a result, the time for total forgetting calculated in the initial cycle has a large effect on the forgetting slopes of successive cycles, and as a result, may affect the final performance measure; namely, a large $D$ should contribute to improved performance since forgetting in successive cycles should be less 
severe. As shown in Figure 4.9, varying the range of $f$ has a relatively insignificant effect on $D$ as compared with varying $L R$. For example, it is not until the initial forgetting rate is as slow as $95 \%(f=0.088$ ) that the time to total forgetting $(D)$ increases by any significant amount. Also, as illustrated in Figure 4.9, it is only at this low range of initial forgetting ( $F R>$ 94\%) that the effect of varying the learning rate affects $D$, whereas, at other levels of $f$, varying $b$ has little effect on the magnitude of $D$. As a result, if the effect of $L R$ on $D$ is to be examined it must be done at low initial forgetting rate levels. The effect of varying $L R$ at an initial forgetting rate of $95 \%$ is shown in leftmost section of Figure 4.9. It appears that $D$ is a concave function throughout the chosen range of $b$ at this initial forgetting rate level, achieving a maximum value when:

$$
\frac{\partial D}{\partial b}=0
$$

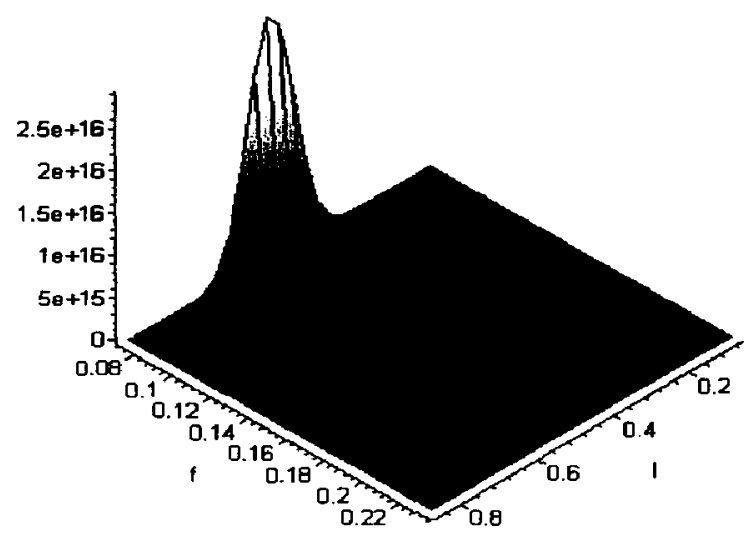

Figure 4.9: 3-dimensional plot of $D$ as a function of $b$ and $f$ with ranges of $0.074<b<0.862$ and $0.074<f<0.234$; where $C$ and $\rho$ are kept constant at 100 and 4 respectively. $(s=0)$ 
Keeping $f$ constant at $0.074(F R=95 \%)$ and $C$ and $\rho$ constant at 100 and 4 respectively gives:

$$
\begin{aligned}
& \frac{\partial D}{\partial b}=\left(10^{\frac{b(1-b) \log (100)}{0.074}}-1\right) \times\left[\frac{4(100)^{1-b}}{1-b}\left(\frac{1}{1-b}-\frac{1}{\log _{100} e}\right)\right] \\
& +\left(\frac{4}{1-b}(100)^{1-b}\right) \times\left[\frac{10^{\frac{b(1-b) \log (100)}{0.074}}}{\log _{10} e}(1-2 b) \times \frac{\log (100)}{0.074}\right]=0 .
\end{aligned}
$$

Solving for $b$ using bisection gives $b=0.4997$, or $L R=70.7 \%$. Therefore, as the learning rate quickens in these circumstances ( $f=0.074, C=100, \rho=4$ ), the time to total forgetting initially increases from a learning rate of $L R=95 \%$ to $L R \rightarrow 70.7 \%$. In other words, when a worker learns quickly $(0.75<b<1)$ he/she also tends to forget quickly, corresponding to a small $D$. This concurs with the findings of Nembhard (2000), and Nembhard and Uzumeri (2000). When the learning rate of the worker slows $(0.25<b<0.75)$, the worker remains at the job for a longer period of time, therefore he/she requires a longer time to totally forget; a maximum $D$ is reached when $b=0.5(L R=70.7 \%$ ). However, if the learning rate of the worker is very slow $0<b<0.25(100 \%<L R<84 \%)$, little is learnt in slow-learning workers; therefore this small amount is forgotten quickly. As a result, for a given value of $f$ and $C$, the value of $D$ is a bell-shaped curve reaching a maximum value at $b=0.5$ ( $L R=$ 70.7). However, the maximum value of $D$ at $L R=70.7 \%$ does not correspond with the findings concluded by Figure 4.4 because the location of this critical point ( $L R^{*}$ ) is not similar to the location of the maximum $C\left(C^{*}\right)$ in Figure 4.4. Furthermore, the range of $L R$ in model $\mathrm{C}$ is $70 \%<L R<90 \%$, this is outside the range that would reveal the bell-shaped function illustrated in Figure 4.9. As a result, the critical point depicted in Figure 4.4 cannot be attributed to the phenomenon of the maximum point illustrated in Figure 4.9 and must be a result of some other model parameter. This was investigated further by averaging the performance measure of $A P T$ within the range of the upfront training policy $(F A P)$. For example, if the performance measure ( $A P T$ ) versus $C$ is plotted again at $F A P-1$ instead of $F A P-0$, the following functions result, see Figure 4.10. 


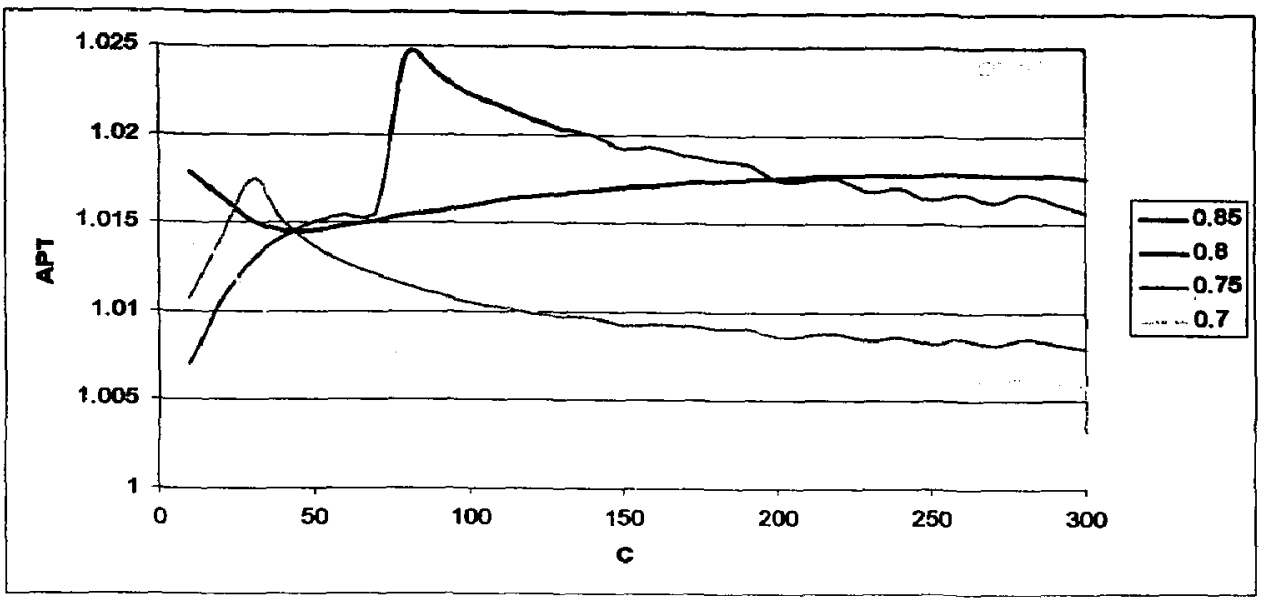

Figure 4.10: Plot of Average processing time ( $A P T$ ) versus $C$ for all $F A P-1$ values averaged across all forgetting rate levels at $\rho=4(s=0, N=2)$

As shown in Figure 4.10, the minimal $A P T$ value occurs at different transfer policy levels. For example, it is clear that the minimal $A P T$ value for a learning rate of $70 \%$ occurs at a maximum $C$ value. However, as learning slows (corresponding to increasingly darker lines) the minimized $A P T$ value is located at shorter transfer times: $70 \%$ learning ( $C^{*}=300$ units), $75 \%\left(C^{*}=300\right.$ units), $80 \%$ ( $C^{*}=10$ units). However, when learning is $85 \%$, the transfer time increases to $C^{*}=50$ units. This observation corresponds to the increase in $C^{*}$ at $L R=85 \%$ in Figure 4.4 to a level nearly as high as when $L R=70 \%$ then decreases to a lowest value of $C^{*}=53.46$ at $L R=90 \%$. Therefore, the behaviour depicted in Figure 4.10 concurs with that of Figure 4.4 that performance tends to improve ${ }^{* * * *}$ greatly before it worsens.

This peculiar behaviour was further investigated by plotting the relationship between time-fortotal-forgetting in the first cycle versus the learning rate of the worker for different levels of initial training, as shown in Figure 4.11. The graph in Figure 4.11 provides a plot of the equation of time to total forgetting $(D)$ against the three initial training policies of model C. Intuitively, the plot of the equation depicts what is expected for the time to total forgetting $(D)$ as the learning rate slows at a setting of $F A P-0$. However, the plot of $D$ corresponding to $F A P-1$ and $F A P-2$ appears to increase after a learning rate of approximately $90 \%$ and reaches a maximum at $95 \%$ before decreasing again. The explanation for this behaviour is given as follows.

\footnotetext{
***** Larger batch sizes are required, suggesting lower forgetting losses; see previous explanation accompanying Figure 4.5.
} 


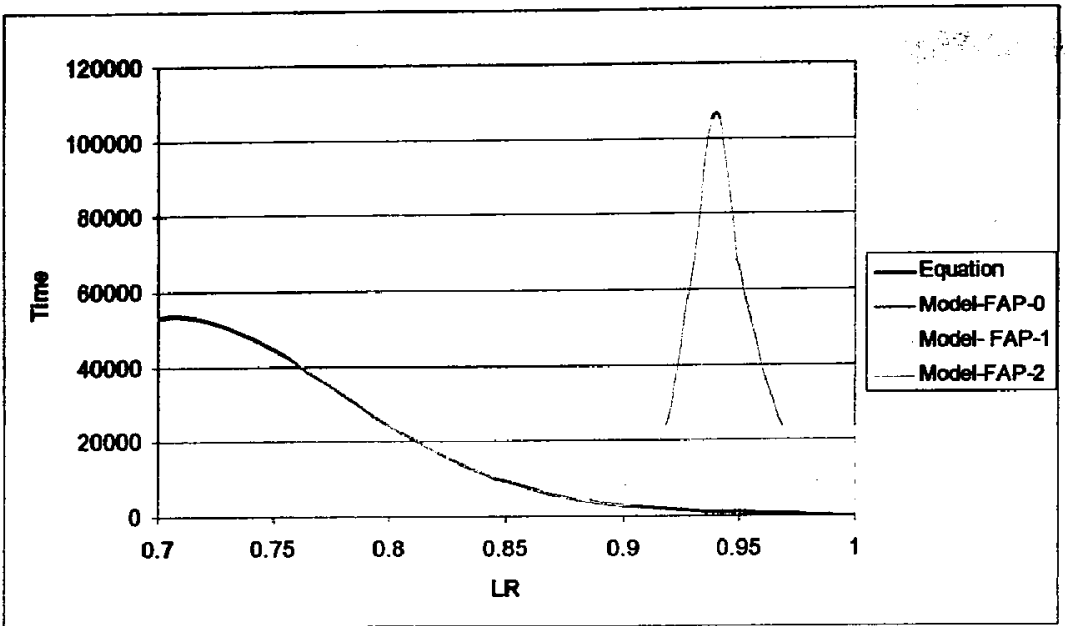

Figure 4.11: Time to total forgetting $(D)$ in the first cycle versus learning rate for different levels of initial training ( $C=100, F R=88 \%, s=0, N=2, \rho=4)$

For example, given $C=100$, the upfront training policy is such that, in a $F A P-1$ or $F A P-2$ situation, the worker in the initial cycle only produces a lot size of 100 in situations of high-learning. At a fast learning rate the lot-size is greater than the number of units needed to reach the low number of units needed to reach standard time $\left(n_{s}\right)$ of the worker. As the learning rate deteriorates, the time for total forgetting decreases, and thus the optimal policy decreases as previously explained (see pg. 158 ) from $L R=70 \%(b=0.515)$ to $L R=87 \%$ $(b=0.201)$. However, if the learning rate decreases to such a point as to cause $n_{s}$ to be greater than the batch size (100), he/she remains at the first station and produces more than the batch size until the standard number of units $n_{s}$ (in a $F A P-1$ situation) or twice the standard number of units $2 n_{s}$ (in a $F A P-2$ situation) are produced. Since such a low learning rate causes the worker to have such a high $n_{s}$, this large amount increases the value of $C^{*}$ greater than if the learning rate of the worker was faster. However, this benefit reaches a maximum value at a certain learning rate (depending on the level of $\rho$ ) and then decreases as the detrimental effect of a low learning rate outweighs the benefit of producing a large initial training batch. Also, as expected, the plot of $F A P-0$ is identical to that of the equation plot since there is no initial training.

However, the learning rate at which the time to total forgetting $(D)$ reaches a maximum is dependent of the value of $\rho$. Since $A P T$ is dependent on $\rho$ it follows that $D$ is also dependent on $\rho$. This relationship is plotted in Figure 4.12. 


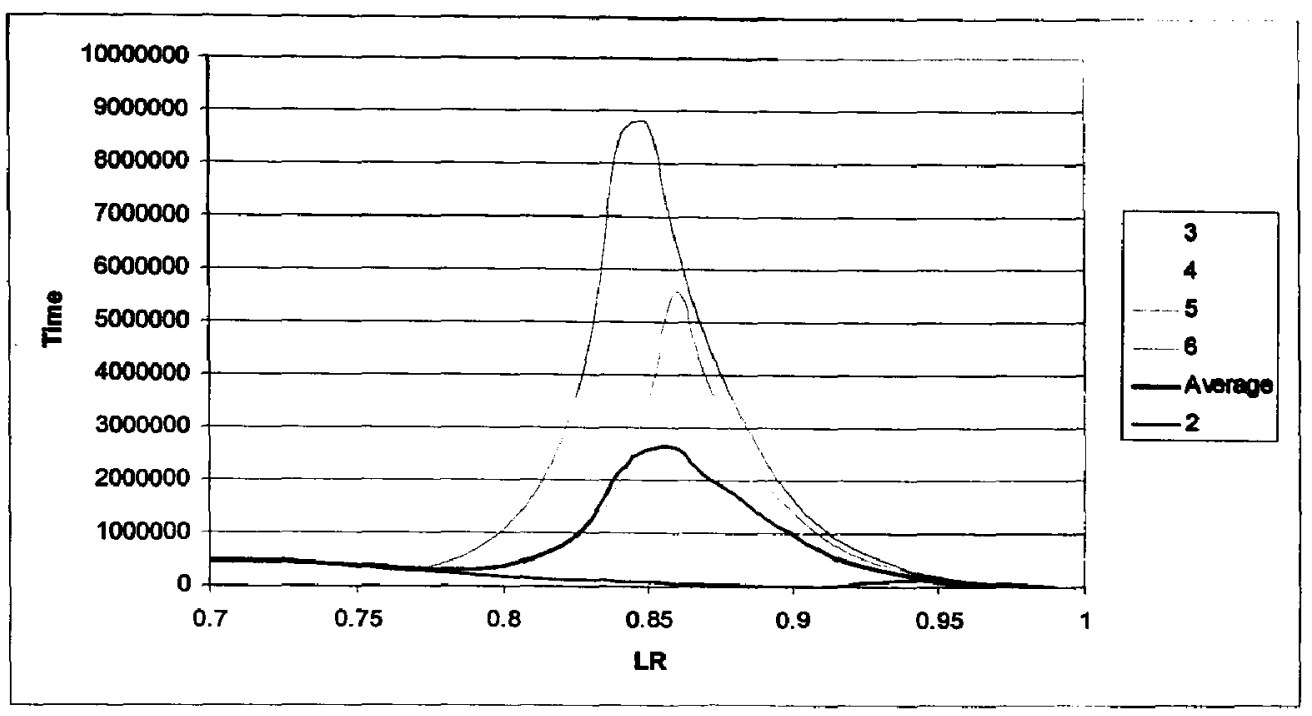

Figure 4.12: Time for total forgetting $(D)$ in the first cycle versus learning rate for different $\rho$-values ( $C=100, F A P-1, F R=88 \%, s=0, N=2$ )

As shown in Figure 4.12, the learning rate at which $D$ reaches a maximum differs according to the value of $\rho$. The maximum value of $D$ is greater and occurs earlier as $\rho$ increases. This is because $n_{s}$ increases exponentially with $\rho$, as given by:

$$
n_{s}=\left(\frac{1}{\rho}\right)^{-\frac{1}{b}}
$$

This causes the experience gained by producing the standard number of units to increase, thereby increasing the length of time required to totally forget acquired experience. Also, as shown in Figure 4.12, the average of the range of the value of $\rho$ yields an optimal learning rate value of approximately $85 \%$. Consequently, this finding explains the reason for the convexity found at an $85 \%$ worker learning rate in Figure 4.4. 


\subsubsection{3 $C^{*}$ versus $\rho$}

The scatter plot of the main effect behaviour of $C^{*}$ with $\rho$ is given in Figure 4.13.

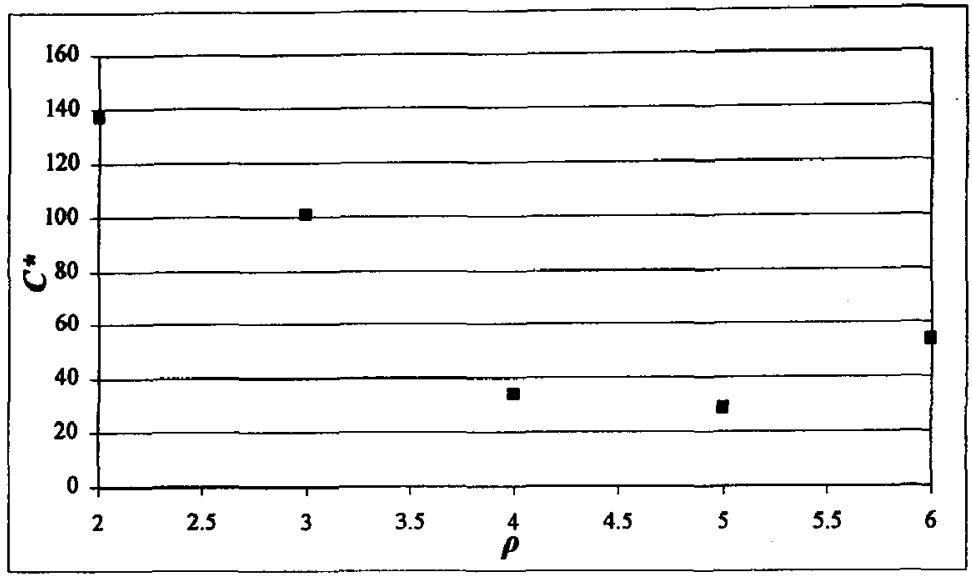

Figure 4.13: Plot of $C^{*}$ versus $\rho$

Figure 4.13 suggests that $C^{*}$ decreases as $\rho$ increases from $\rho=2$ to 5 , and then increases at $\rho=6$. Again, examining the general overall trend in $A P T$ versus $\rho$ and $C$ does not necessarily explain why the plot in Figure 4.13 behaves as it does. For example, in Figure 4.14, a 3-D plot of the time to total forgetting $(D)$ as a function of $\rho$ and $C$ was constructed.

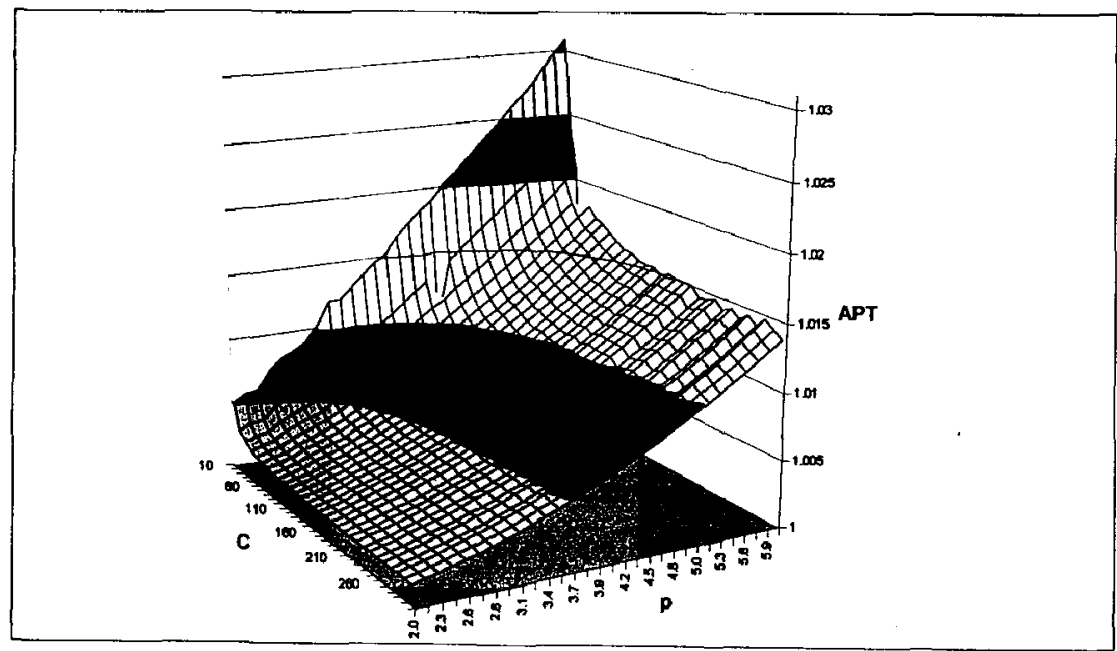

Figure 4.14: 3-dimensional plot of average processing time ( $A P T)$ as a function of $\rho$ and $C$ with tanges of $2<\rho<6$ and $100<C<300$; where $b$ and $f$ are kept constant at $0.152(L R=90 \%)$ and $0.322(F R=80 \%)$ respectively $(F A P-0, s=0, N=2)$ 
As shown in Figure 4.14, and in most instances of $\rho$, performance worsens as $\rho$ increases. This is intuitive because more learning is required simply because the initial unit processing time is greater. However, an exception to this is found in cases when learning is fast and forgetting is slow. For example, in Figure 4.15 , when the learning rate is $70 \%$, forgetting rate is $95 \%$, and $C=10$, it appears that the average processing time increases in the range $2<\rho<3$, reaches a maximum at $\rho \approx 3$, and actually decreases when $\rho>3$.

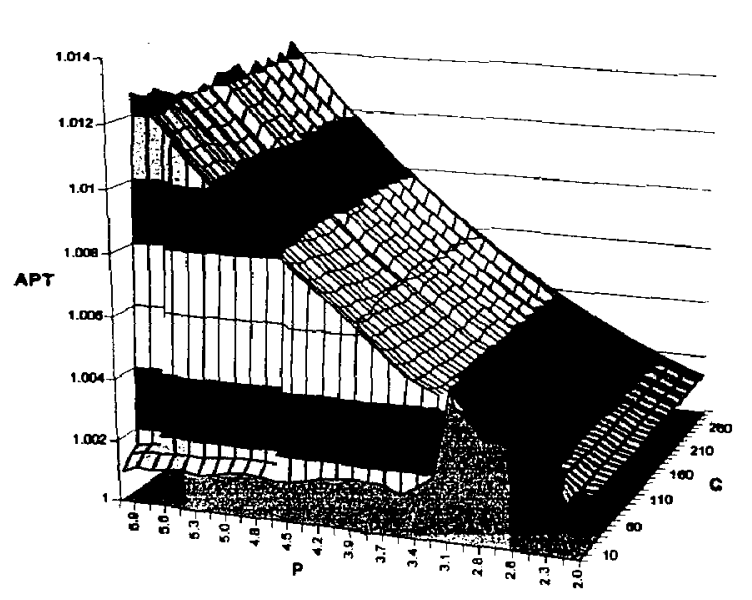

Figure 4.15: 3-dimensional plot of average processing time ( $A P T)$ as a function of $\rho$ and $C$ with ranges of $2<\rho<6$ and $100<C<300$; where $b$ and $f$ are kept constant at $0.515(L R=70 \%)$ and $0.074(F R=95 \%)$ respectively. $(F A P-1, s=0, N=2)$

Since the model $\mathrm{C}$ search procedure would have found the exceptions to the $A P T$ behaviour with respect to $\rho$ and $C$, as found in the parameters used to construct Figure 4.15 , it is logical that the minimized values of $C^{*}$ found in Figure 4.13 do not necessarily conform to 'average' behaviour (as in Figure 4.14) but are equal to extreme values of $A P T$ as shown in specific combinations of parameters such as $F A P-1, L R=70 \%, F R=95 \%, C=10$, and $s=0$.

\subsubsection{4 $C^{*}$ versus $F R$}

The scatter plot of the main effect behaviour of $C^{*}$ versus forgetting rate is given in Figure 4.16. Figure 4.16 suggests that $C^{*}$ decreases as the worker forgetting rate slows from a high forgetting rate of $F R=85 \%$ to low forgetting of $F R=95 \%$. 


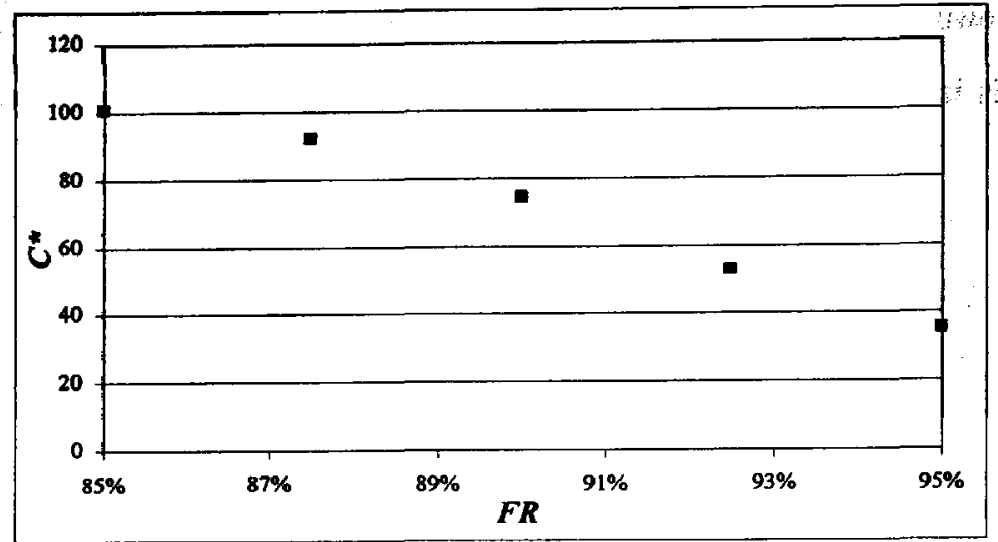

Figure 4.16: Plot of $C^{*}$ versus $F R$

This is as expected because as the worker forgetting rate slows, the shorter batch sizes are allowed because total forgetting losses are less when the initial forgetting rate is slower. As previously mentioned, the time for total forgetting $(D)$ calculated in the initial cycle has a large effect on the forgetting slopes of successive cycles and is dependent upon the initially specified forgetting rate. The value of $D$ remains constant throughout successive cycles. However, as shown in Figure 4.9, varying the range of $f$ has a relatively insignificant effect on $D$ as compared with varying $b$. Nonetheless, varying the initial worker forgetting rate has a detectable affect on the average processing time, see Figure 4.17.

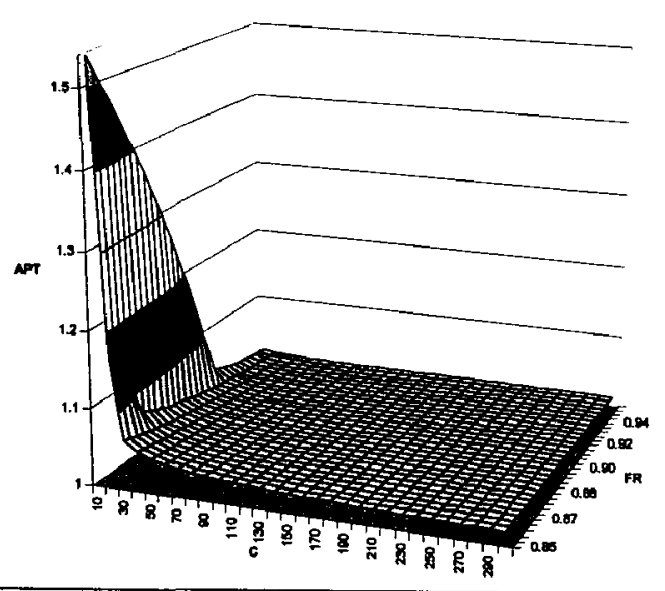

Figure 4.17: 3-dimensional plot of average processing time ( $A P T)$ as a function of $F R$ and $C$ with ranges of $85 \%<F R<95 \%$ and $100<C<300$; where $b$ and $\rho$ are kept constant at $0.322(L R=80 \%)$ and 4 respectively $(F A P-0, s=$ $0, N=2$ ) 
As shown in Figure 4.17, the feasibility of transferring after smaller lot sizes increases as the worker forgetting rate slows.

\subsubsection{Main effect analysis of $F A P^{*}$}

The main effect results (which are the information for the plots) for $F A P^{*}$ are given in Table 4.11.

Table 4.11: The main effect of each input variable versus level of $F A P^{*}$

\begin{tabular}{|c|c|c|}
\hline Input variable & Level & $F A P^{*}$ \\
\hline \multirow[t]{5}{*}{ Degree of task similarity $(s)$} & 0.0 & 0.788 \\
\hline & 0.2 & 0.844 \\
\hline & 0.4 & 0.972 \\
\hline & 0.6 & 1.148 \\
\hline & 0.8 & 1.252 \\
\hline \multirow{5}{*}{$\begin{array}{l}\text { Initial processing time to } \\
\text { standard time ratio }(\rho)\end{array}$} & 2 & 1.296 \\
\hline & 3 & 1.136 \\
\hline & 4 & 0.804 \\
\hline & 5 & 0.876 \\
\hline & 6 & 0.892 \\
\hline \multirow{5}{*}{$\begin{array}{l}\text { Worker learning rate }(L R) \text {, } \\
\text { where } L R=100 \times 2^{-b}\end{array}$} & $70 \%$ & 1.224 \\
\hline & $75 \%$ & 0.912 \\
\hline & $80 \%$ & 1.072 \\
\hline & $85 \%$ & 0.904 \\
\hline & $90 \%$ & 0.892 \\
\hline \multirow{5}{*}{$\begin{array}{l}\text { Worker forgetting rate }(F R) \text {, } \\
\text { where } F R=100 \times 2^{f}\end{array}$} & $95 \%$ & 1.212 \\
\hline & $92.5 \%$ & 1.068 \\
\hline & $90 \%$ & 0.932 \\
\hline & $87.5 \%$ & 0.884 \\
\hline & $85 \%$ & 0.908 \\
\hline
\end{tabular}

\subsubsection{1 $F A P^{*}$ versus $s$}

The scatter plot of the main effect behaviour of $F A P^{*}$ with degree of task similarity $(s)$ is given in Figure 4.18. 


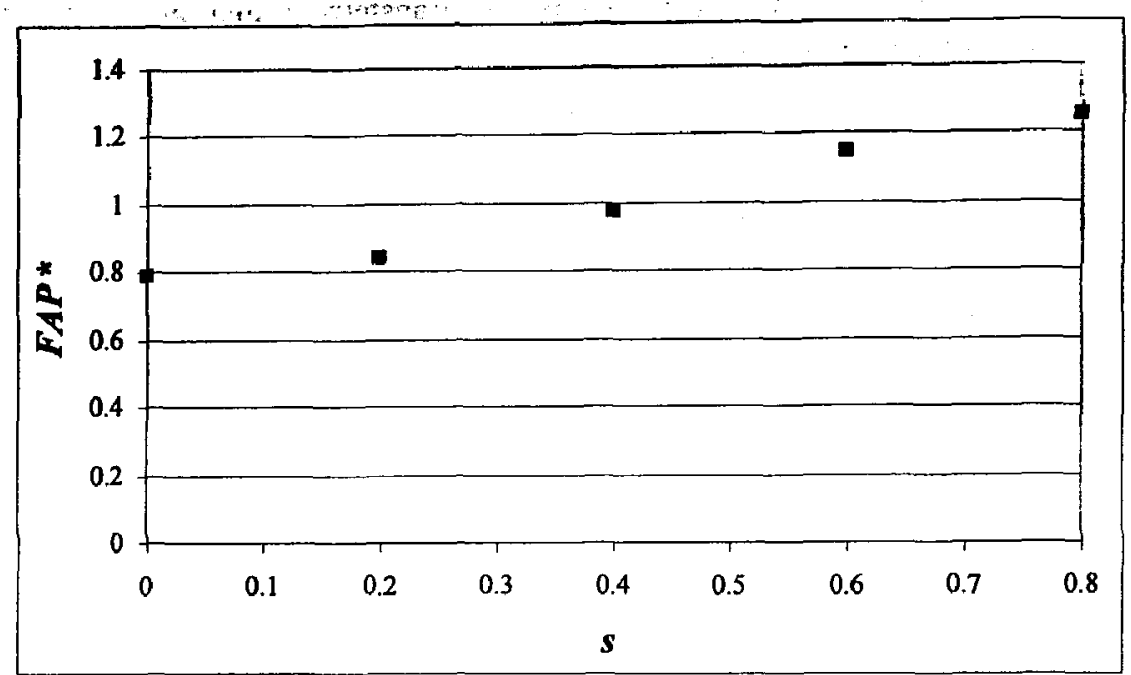

Figure 4.18: Plot of $F A P^{*}$ versus $s$

Figure 4.18 suggests that $F A P^{*}$ increases as the degree of task similarity increases $(s)$. As previously mentioned, examining one main effect may not fully explain the reasons for the above $F A P^{*}$ values. For example, the raw results of model $\mathrm{C}$ (see section 7.7 ) oscillate between an optimal upfront training policy of $F A P-0, F A P-1$, and $F A P-5$. As a result, since Figure 4.18 just represents averaged results across all the specified levels of the parameters, the upward trend simply indicates more $F A P-5$ results than $F A P-0$ or $F A P-1$ results.

For example, in the particular parameter setting used to construct Figure 4.19, an upfront training policy of $F A P-5$ always seems optimal.

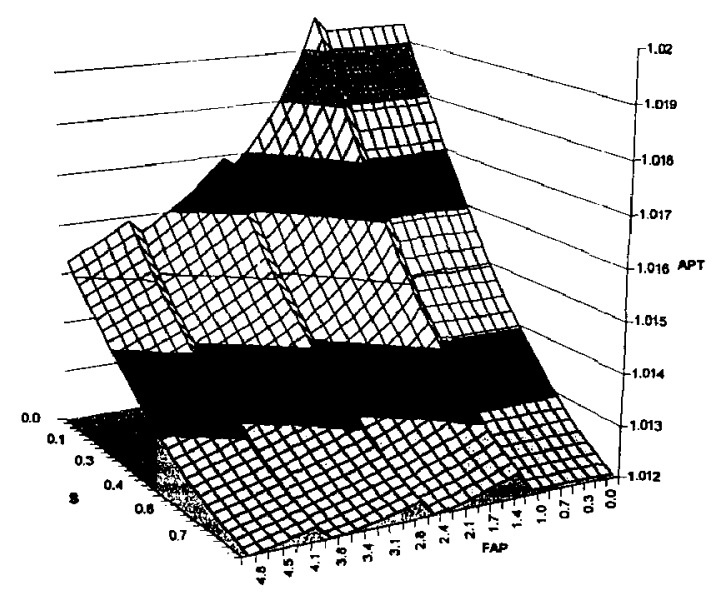

Figure 4.19: 3 -dimensional plot of average processing time $(A P T)$ as a function of $s$ and $F A P$ with ranges of $0<s<0.8$ and $0<F A P<5$; where $b$ and $f$ are kept constant at $0.139(L R=80 \%)$ and $0.152(F R=90 \%)$ respectively $\quad(s=$ $0, \rho=4, N=2, C=100$ ) 
However, if only one parameter such as the batch transfer size $(C)$ is changed from $C=100$ (Figure 4.19) to $C=250$ (Figure 4.20), the plots suggest that a smaller upfront training policy such as $F A P-1$ or $F A P-0$ (instead of $F A P-5$ ) is the preferred upfront training policy, see Figure 4.19 versus Figure 4.20.

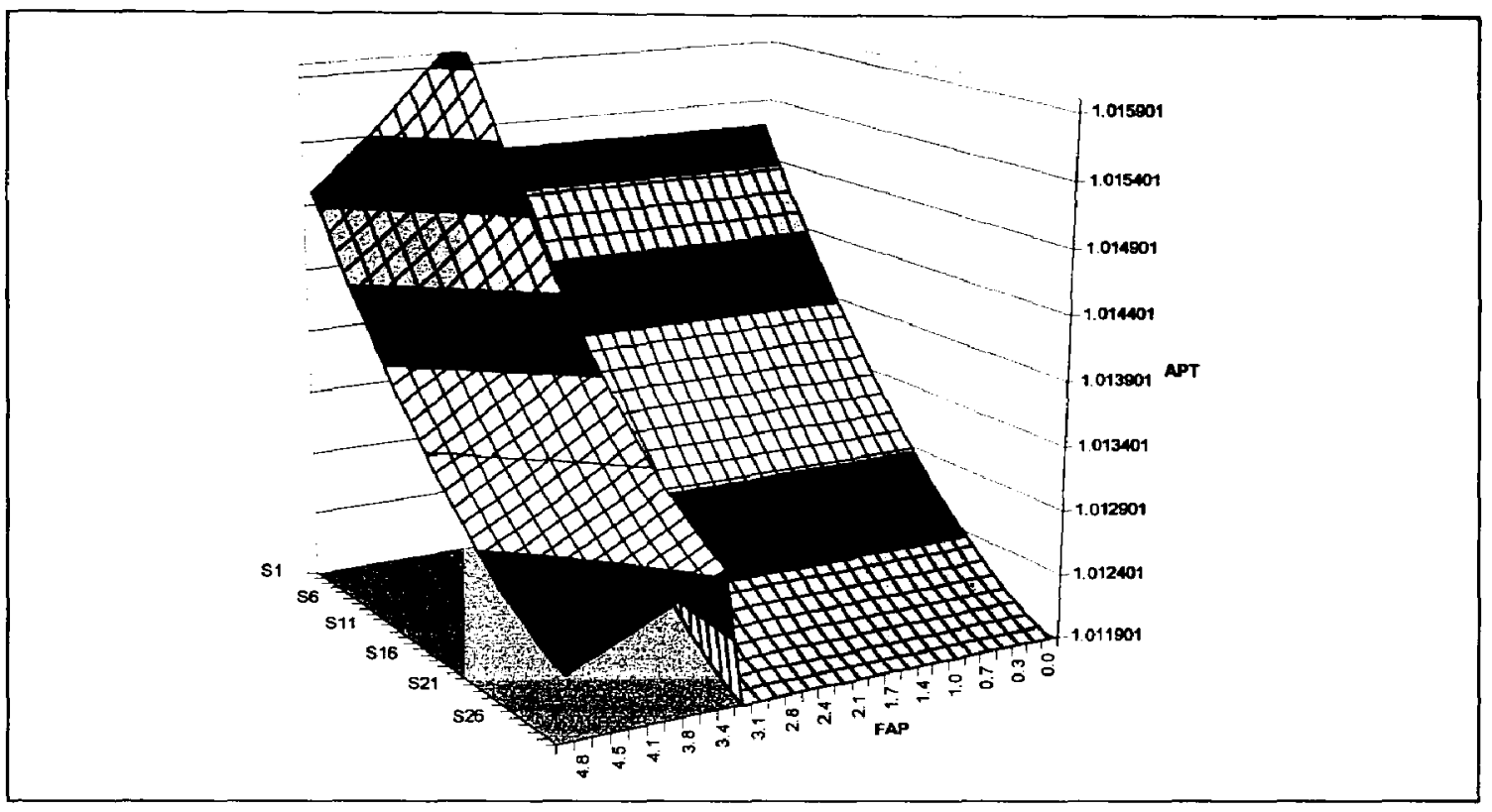

Figure 4.20: 3-dimensional plot of average processing time ( $A P T$ ) as a function of $s$ and $F A P$ with ranges of $0<s<0.8$ and $0<F A P<5$; where $b$ and $f$ are kept constant at $0.139(L R=80 \%)$ and $0.152(F R=90 \%)$ respectively $\quad(s=$ $0, \rho=4, N=2, C=250$ )

As shown later, the multivariate function $g(s, \rho, L R, F R)=\left(F A P^{*}, C^{*}\right)$ accounts for all these unique optimal points that may not be initially obvious by only examining the main effect but may occur at particular interaction combinations of all the parameters listed in Table 3.5. As a result, the remaining main effect of each input variable versus levels of $F A P^{*}$ (Table 4.11) are plotted with their general trend lines and are included in Figure 4.21, Figure 4.22, and Figure 4.23 without further analysis since their variation above and below $F A P-1$ is so minimal that a detailed interaction analysis would be unwieldy given the minimal benefit of obtaining this information. Furthermore, any overlooked interactions between any of the factors is accounted for in the multivariate function that follows and their application is explicitly demonstrated in the numerical example supporting the use of this multivariate function. 


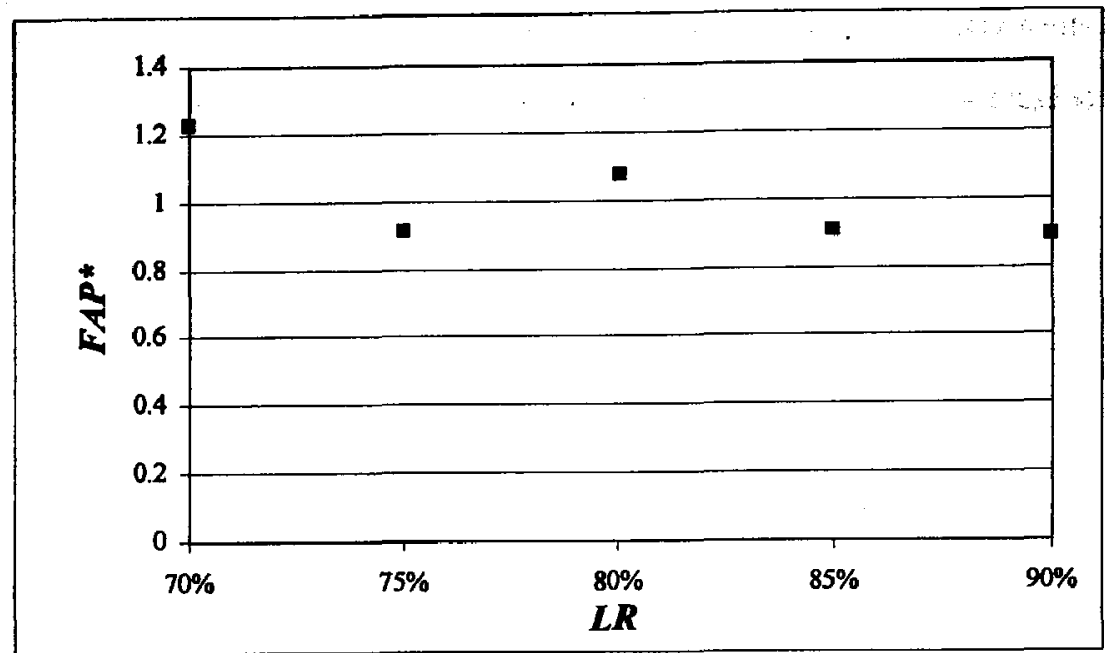

Figure 4.21: Plot of $F A P^{*}$ versus $L R$

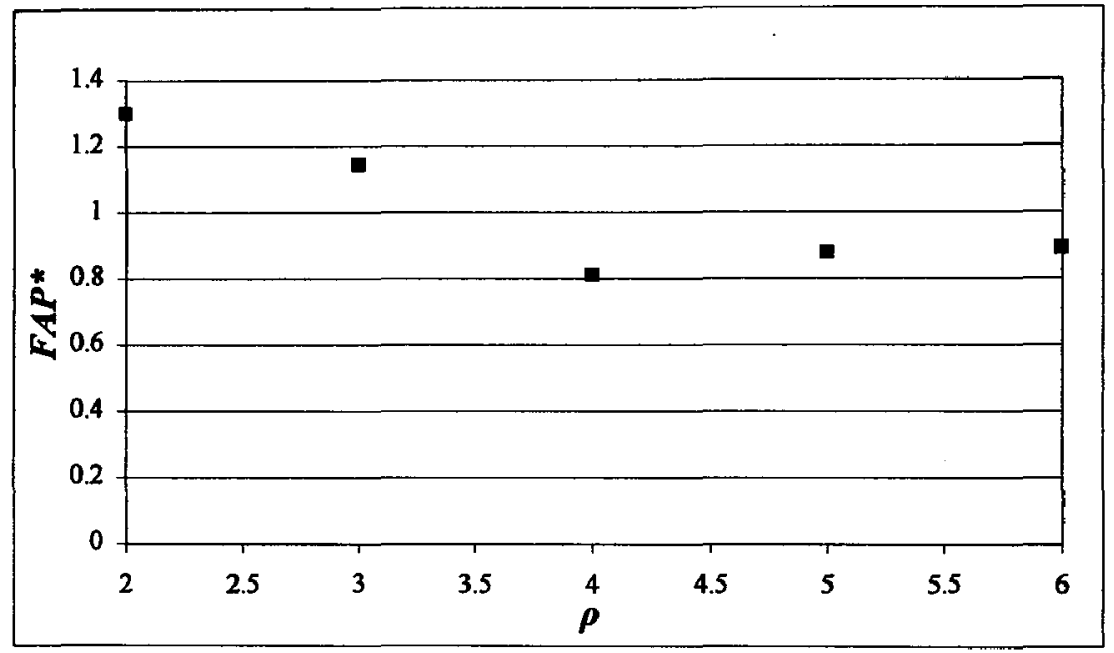

Figure 4.22: Plot of $F A P^{*}$ versus $\rho$

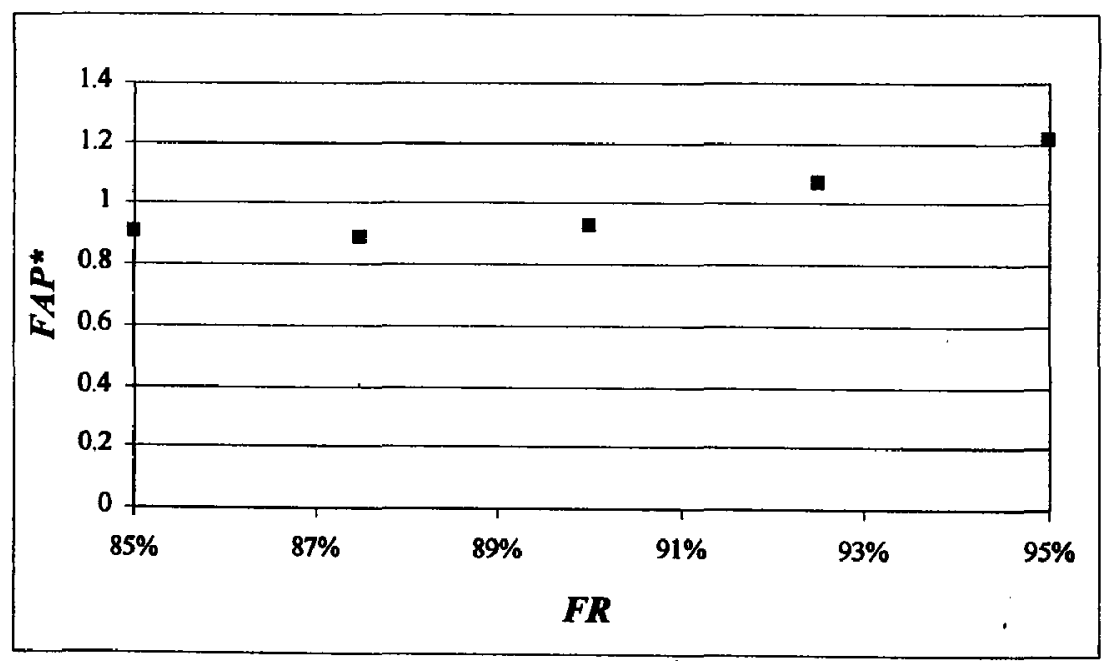

Figure 4.23: Plot of $F A P^{*}$ versus $F R$ 


\subsubsection{Logistic regression equations and numerical example}

\subsubsection{Prologue}

The distribution of the 625 output variables for both the optimal batch transfer size policy ( $\left.C^{*}\right)$ and the optimal level of upfront training policy $\left(F A P^{*}\right)$ are illustrated by histogram plots given in Figure 4.24 and Figure 4.25.

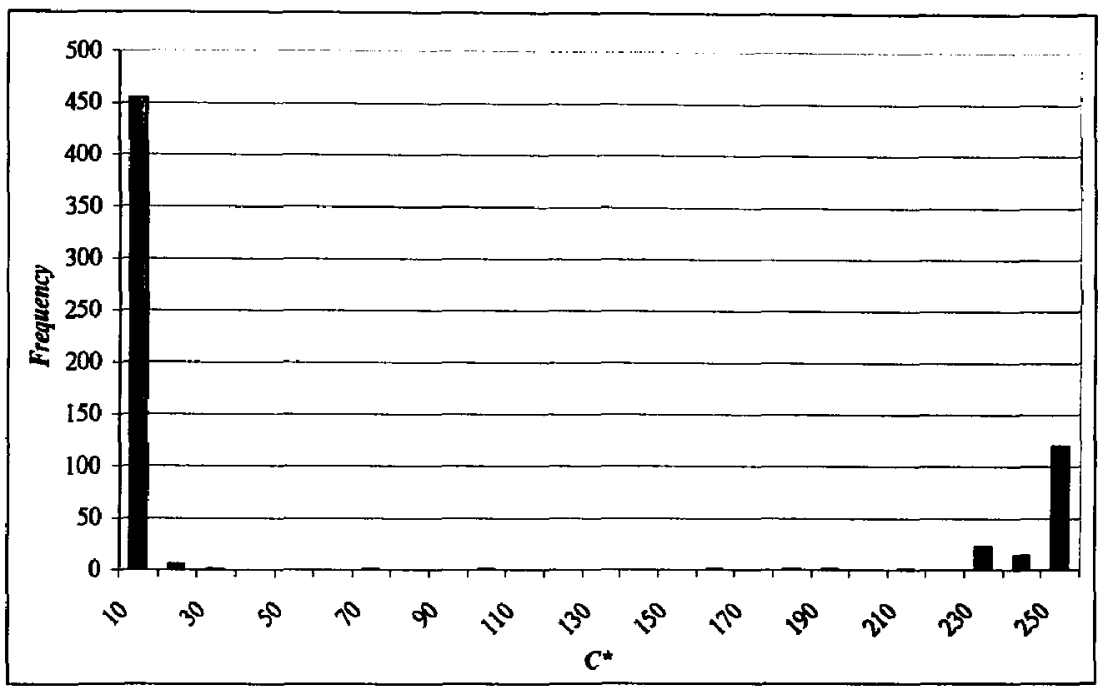

Figure 4.24: Histogram plot of the distribution of $C^{*}$ values

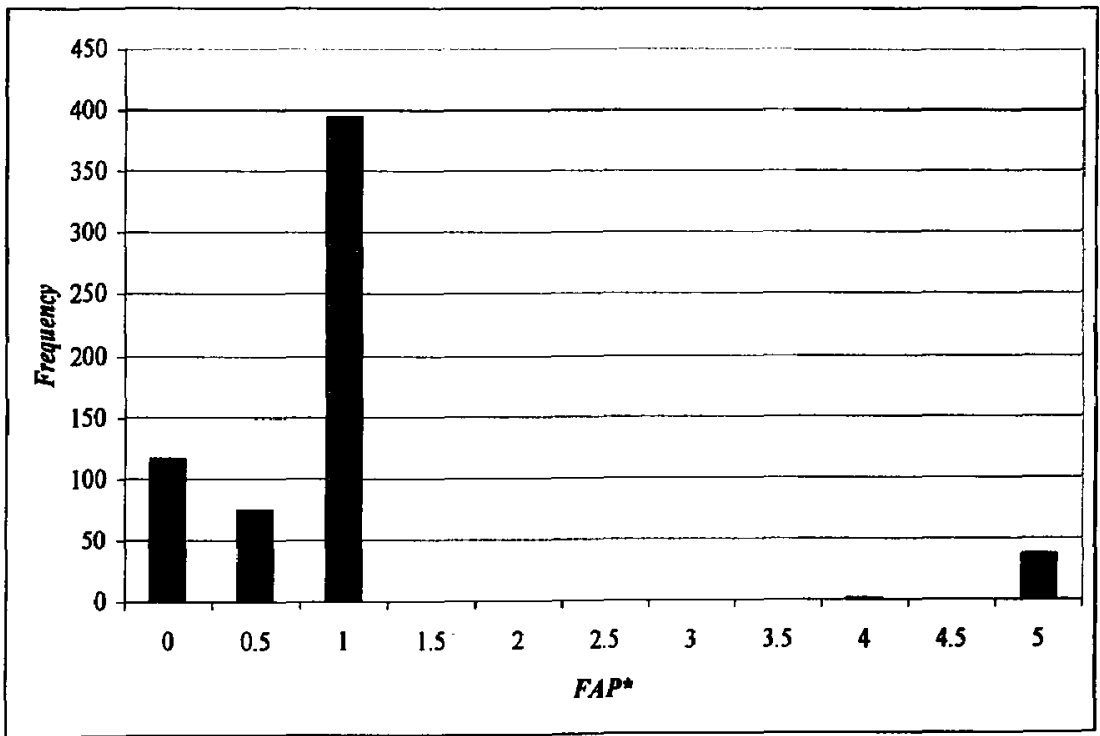

Figure 4.25: Histogram plot of the distribution of $F A P^{*}$ values 
As shown in Figure 4.24 and Figure 4.25, the distributions of $C^{*}$ and $F A P^{*}$ are irregular with few or no data points in the middle sections of the specified ranges. The optimal values appear to cluster at the extremes of both specified ranges of $C$ and $F A P$. The reason for this unusual distribution is described as follows. Note that the data in Figure 4.24 and Figure 4.25 are 'optimized' values of $F A P$ and $C$ that minimize the performance measure of the DRC system in question, namely: average processing (service) time ( $A P T$ ) calculated over approximately 3000 jobs per station. In order to explain why the distributions in Figure 4.24 and Figure 4.25 have these densities, it may help to plot four sections ${ }^{* * *}$ of the behaviour of the performance measure with respect to $C$ and $F A P$, see the graphs in Figure 4.26(a), Figure 4.26(b), Figure 4.27(a), and Figure 4.27(b).

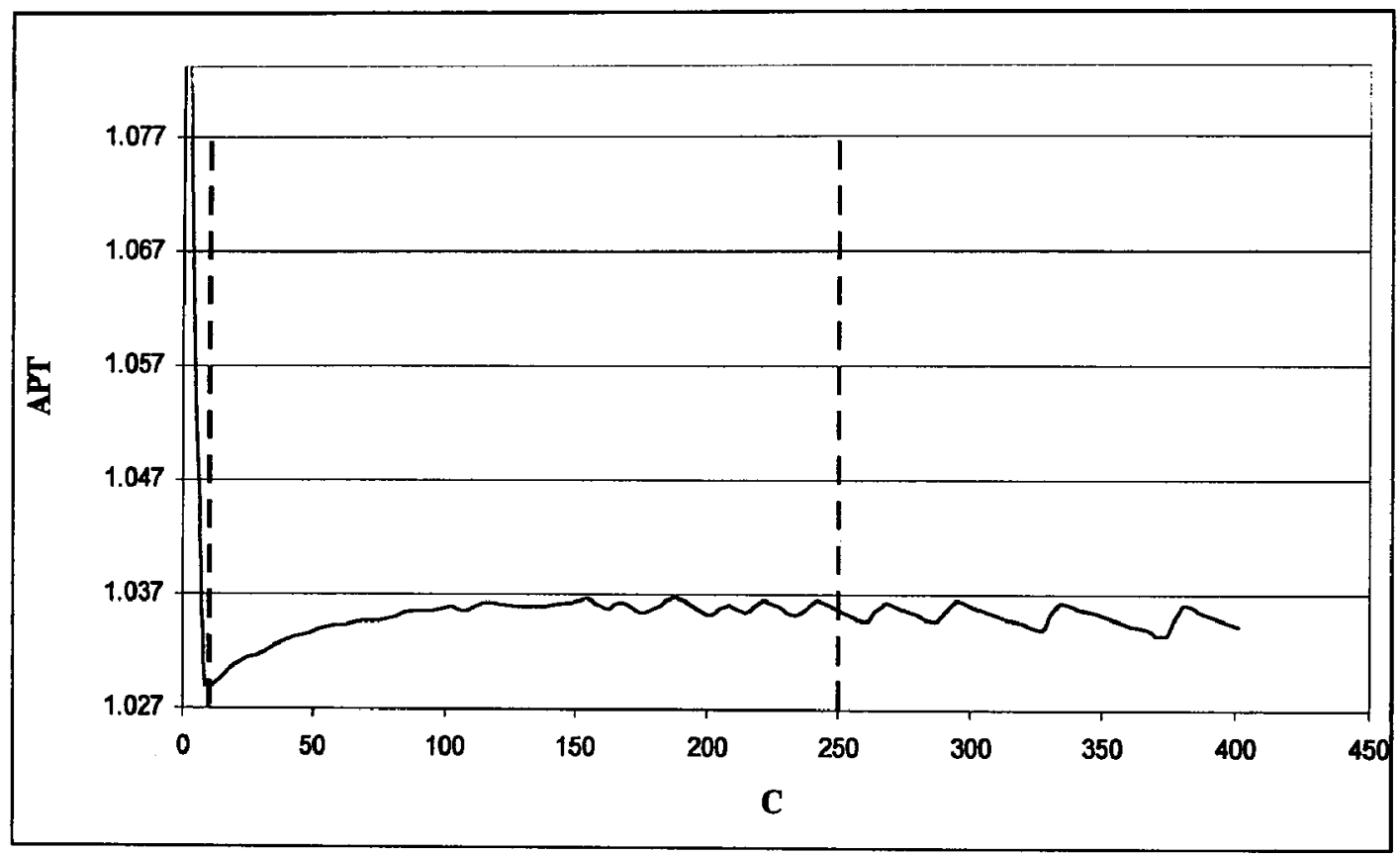

Figure 4.26(a): Plot of $A P T$ versus $C(s=0, N=2, \rho=5, L R=80 \%$, $F R=85 \%$ )

\footnotetext{
**** Combination of factors allowing for a two-dimensional plot.
} 


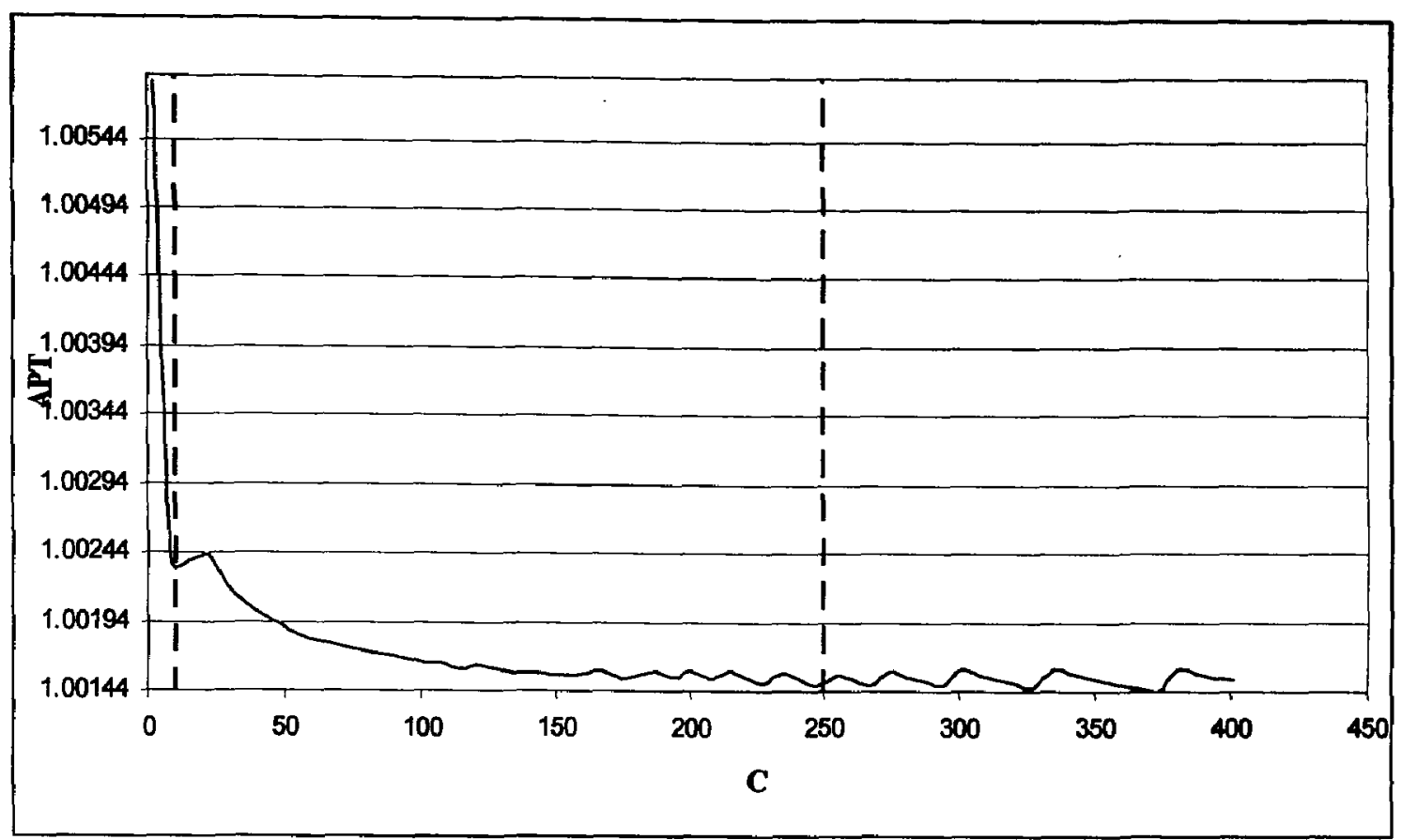

Figure 4.26(b): Plot of $A P T$ versus $C(s=0, N=2, \rho=2, L R=90 \%$, $F R=95 \%)$

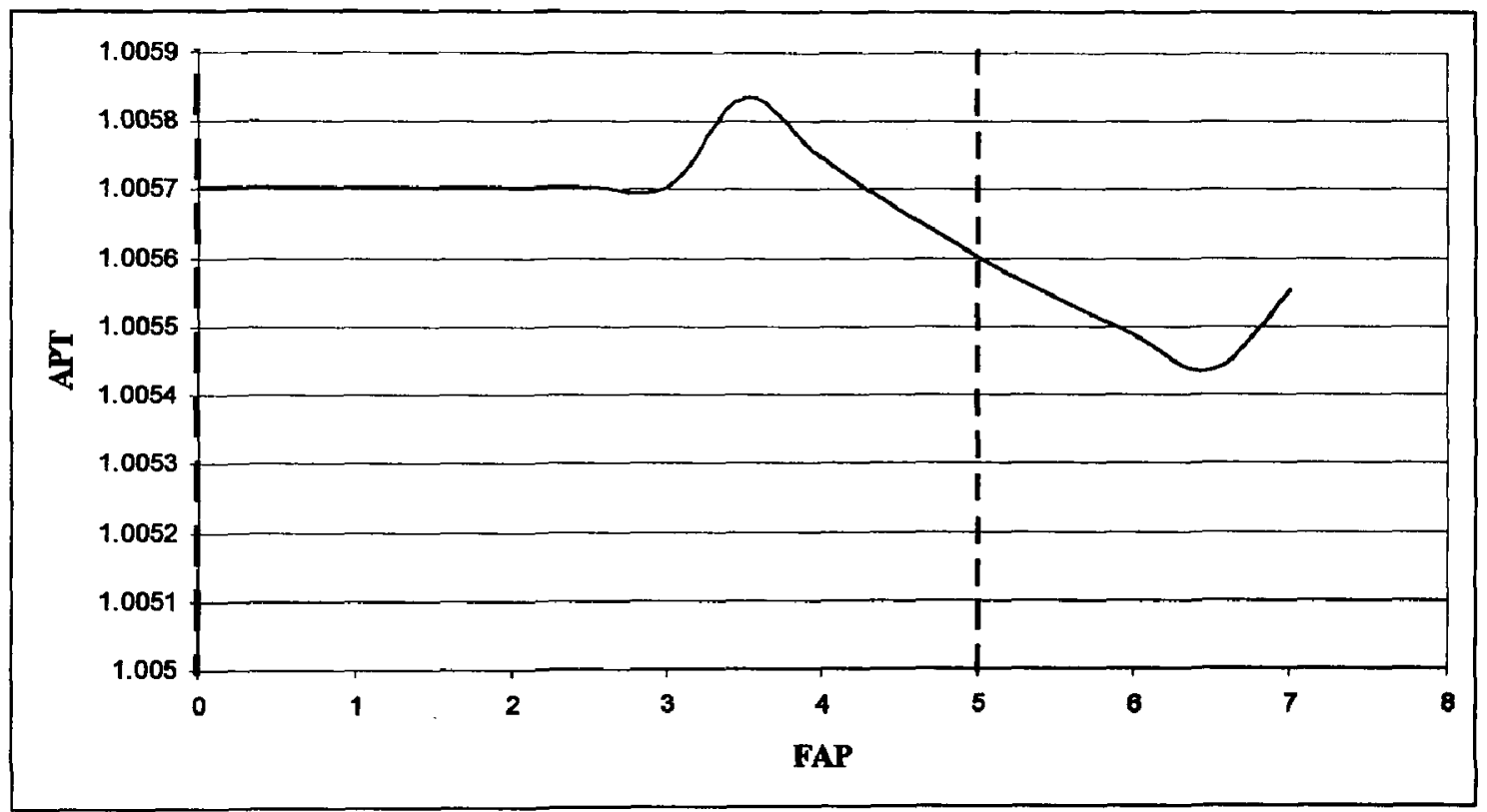

Figure 4.27(a): Plot of $A P T$ versus $F A P(s=0, N=2, \rho=3, L R=80 \%$, $F R=95 \%$ ) 


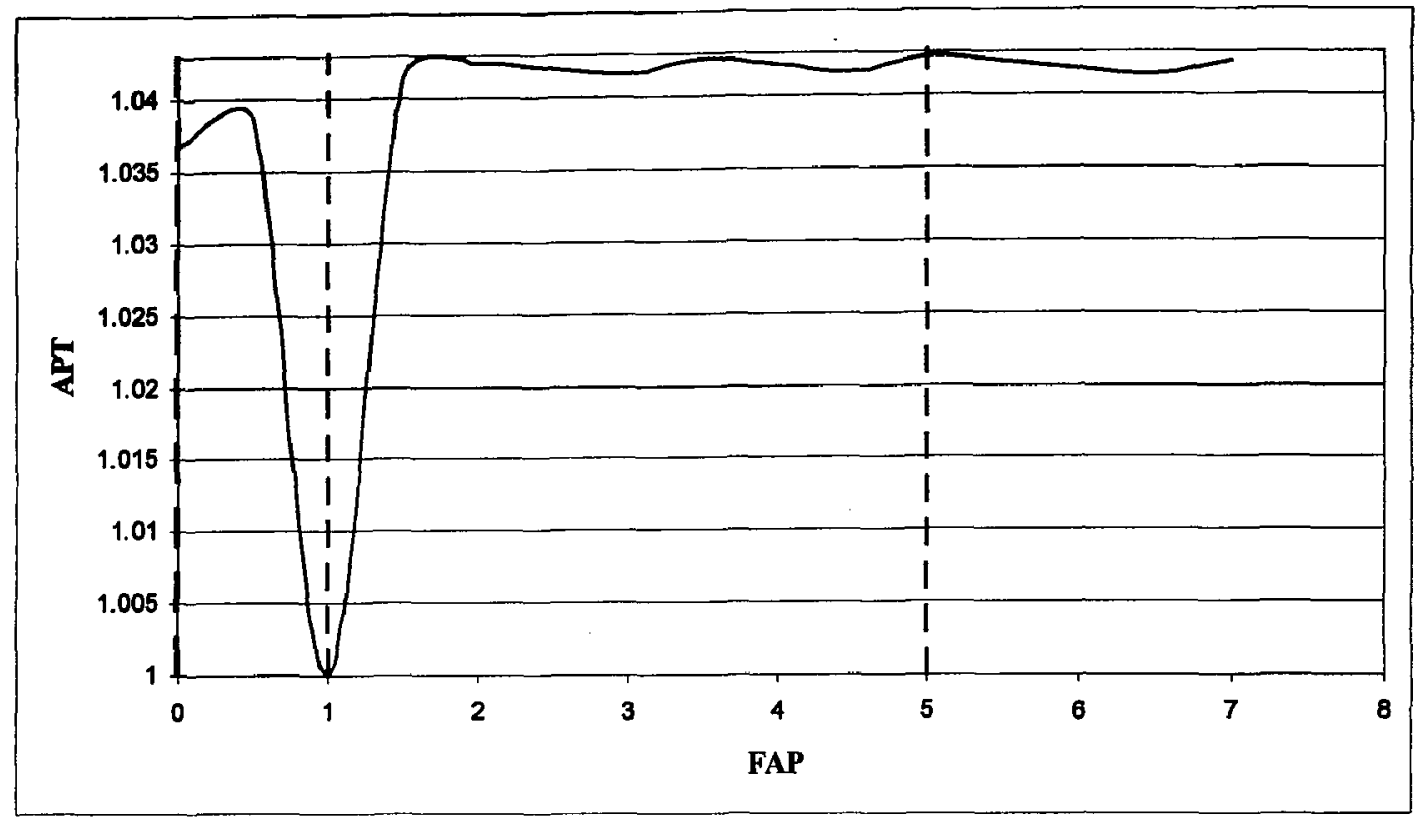

Figure 4.27(b): Plot of $A P T$ versus $F A P(s=0, N=2, \rho=6, L R=80 \%$, $F R=92.5 \%$ )

As shown in the above graphs, depending on the levels of the input variables $(s, \rho, L R, F R)$, the shape of function of $A P T$ versus $C$ and $F A P$ varies greatly within certain variable settings. However, regardless of the shape of the function, the minimum value of the performance measure usually appears at the extremes of the ranges. See the vertical dotted range bars in the graphs. For example, in Figure 4.26(a), the minimum value is located at the leftmost range of $C, C^{*}=10$, and in Figure 4.26(b) (a change in the levels of $\rho, L R$, and $F R$ from the settings used in Figure 4.26(a)), the minimum value is located at the rightmost range of $C$, $C^{*}=250$. Similarly, with the graphs of $A P T$ versus FAP (Figure 4.27(a) and Figure 4.27(b)), the minimum value is located at the rightmost range of $F A P, F A P^{*}=5$ in Figure 4.27(a), and in Figure 4.27(b) (a change in the levels of $\rho$, and $F R$ from settings used in Figure 4.27(a)), the minimum value is located at the leftmost range of $F A P, 0 \leq F A P^{*} \leq 1$.

As a result, fitting a function to the data using regular non-linear multiple regression was not feasible because it resulted in a function that provided outputs that occurred in the middle sections of the range. Namely, the function in the middle of the range was not representative of the actual result data, yet, in the process of regression, it was still recognized because the regression technique had to account for just a few data points occurring in the middle sections of the ranges. The problem of fitting a function to the peculiar distributions was circumvented by 
excluding a small fraction of the data in each distribution and re-fitting the data using binary logistic multiple regression (professor Gharghouri, Dr. Kolasa, and Dr. Todorow, personal communication, 2003). Namely, the use of binary logistic regression necessitated creating a set of data with only two outcomes: ' 1 ' or ' 0 '. In the $C^{*}$ output data the ' 1 ' data corresponded to DRC shop situations where the optimal $C^{*}$ equalled 250 units, whereas the ' 0 ' data corresponded to $C^{*}=10$.

Likewise, in the $F A P^{*}$ output data, the '1' data corresponded to DRC shop situations where the optimal $F A P^{*}$ equalled five times the standard number of units and the ' 0 ' data corresponded

to $0 \leq F A P^{*} \leq 1$, a policy ranging between no upfront training to one times the standard number of units. The adoption of logistic multiple regression necessitated excluding a total of 52 data points located in the middle sections of the distributions of $C^{*}$ and $F A P^{*}$. As a result, only $91.7 \%(573 / 625)$ of the total possible DRC situations as specified in Table 3.5 were accounted for by using this regression technique. This compromise was deemed as the preferred trade-off because the alternative of including all the data would result in either erroneous or irrelevant results.

Consequently, due to the distribution of the output variables, the values were fitted to the functions in (3.10) and (3.11) using binary logistic multiple-regression. The following section provides a pseudo DRC shop situation and demonstrates the application of the logistic regression equations with a numerical example.

\subsubsection{Numerical example - the application of the logistic equations}

\subsection{The logistic equations}

As previously discussed, the results of model $C$ suggest that the optimal batch transfer policy $\left(C^{*}\right)$ is a binary result with ' 1 ' corresponding to $C^{*}=250$ and ' 0 ' corresponding to $C^{*}=10$. The calculation of the optimal batch transfer policy $\left(C^{*}\right)$ is illustrated in the following numerical example. The goodness-of-fit tests for both logistic equations in (4.15) and (4.18) are 
given in the appendix section 7.8.

The binary logistic regression equation for the optimal batch transfer policy $\left(C^{*}\right)$ for model $C$ was calculated using $\mathrm{SAS}^{\circ}$ statistical computer software and is given as follows (Cody and Smith, 1997):

Logistic Equation $\left(C^{*}\right)=$

$=\log \left(\right.$ odds of $\left.C^{*}=250\right)$

$=59.1698-1.8838 \times \rho-6.5048 \times s-42.5111 \times F R-18.0364 \times L R$

where the 'odds' is defined by:

$$
\text { odds }=\frac{P}{1-P}
$$

and $P$ is the probability.

Therefore, from (4.16), $P$ is given by:

$$
P=\frac{o d d s}{1+o d d s}
$$

Similarly, since the results of model $\mathrm{C}$ also suggest that the optimal upfront training policy is a binary result with ' 1 ' corresponding to $F A P^{*}=5$ and ' 0 ' corresponding to $0 \leq F A P^{*} \leq 1$, the optimal upfront training policy $\left(F A P^{*}\right)$ is calculated in a similar manner.

The binary logistic regression equation for the optimal upfront training policy $\left(F A P^{*}\right)$ for model $\mathrm{C}$ was calculated using $\mathrm{SAS}^{\circ}$ statistical computer software and is given as follows (Cody and Smith, 1997):

Logistic Equation $\left(F A P^{*}\right)=$

$=\log \left(\right.$ odds of $\left.F A P^{*}=5\right)$

$=3.0171-1.5797 \times \rho+1.5667 \times s+6.5473 \times F R-9.7559 \times L R$

As a result, the equations in (4.15) and (4.18) could assist the manager of a DRC shop in 
determining, within a given probability, whether the optimal batch transfer size $\left(C^{*}\right)$ and the optimal upfront training policy ( $F A P^{*}$ ) should be set at their minimum or maximum ranges.

\subsection{Description of the pseudo DRC shop problems}

\subsection{DRC shop problem 1}

Consider a DRC shop that utilizes one worker to assemble two types of musical instruments: $V_{1}$ and $V_{2}$. Experimental data suggests that the worker has a learning rate of $87.5 \%$ for this particular set of tasks. ${ }^{4+\dagger \dagger}$ The tasks of assembling the musical instruments $V_{1}$ and $V_{2}$ consist of sub-task attributes given as follows: $\operatorname{Task}_{v_{\mathrm{l}}}=\{A, B, C, D, Q, Z\} \quad$ and Task $_{V_{2}}=\{B, C, E, F\}$. The time to produce the first musical instrument $V_{1}$ or $V_{2}$ was calculated as $y(1)=240$ minutes. ${ }^{\ddagger \ddagger \ddagger}$ The worker is transferred back and forth between stations and some experience is lost during transfers; the rate at which this experience is forgotten is $19 \%$, or a forgetting rate of $81 \% .^{\S \S \S}$ The manger of the musical instrument shop has set an assembly time standard of $y_{s}=114.3$ minutes. Using the logistic equations in (4.15) and (4.18), what will the manager suggest as an optimal batch transfer size policy $\left(C^{*}\right)$ and upfront training policy $\left(F A P^{*}\right)$ in order to minimize the DRC shop performance measure of average processing (service) time ( $A P T$ ) calculated over approximately 3000 jobs per station?

\subsection{DRC shop problem 2}

Suppose the current employee of the musical instrument DRC shop resigned and a new employee from another division was hired. His learning rate was obtained by performing similar work in the other division and was measured as $89 \%$, a slower learning rate than the previous employee. The manger has also replaced the assembly of musical instrument $V_{2}$ with a new and

\footnotetext{
${ }^{\dagger \dagger \dagger}$ Musical instrument learning rates provided by Dar-E1, 2000.

¥\#¥ Musical instrument initial assembly times provided by Dar-El, 2000.

$\$ \$ \S \S$ Guidelines on forgetting rate selection are not available (Kher, 2000).
} 
improved design $V_{3}$, an instrument that consists of fewer sub-task attributes than

$V_{2}:$ Task $_{V_{3}}=\{B, C, Z\}$. Also, the manager reduced the assembly standard time to $y_{s}=43.6$ minutes. Again, using the logistic equations in (4.15) and (4.18), what will the manager suggest as a new optimal batch transfer size policy $\left(C^{*}\right)$ and upfront training policy $\left(F A P^{*}\right)$ in order to minimize the DRC shop performance measure of average processing (service) time ( $A P T$ ) calculated over approximately 3000 jobs per station?

\subsection{Solution of DRC shop problem 1}

Initially, before any calculations can begin, the parameters $s, \rho, L R$, and $F R$ must be determined by examining the problem statement. The learning and forgetting rate of the worker are stated explicitly and are as follows: $L R=87.5 \%$ and $F R=81 \%$. The initial processing time to standard processing time of the task $(\rho)$ is given by:

$$
\rho=\frac{y(1)}{y_{s}}=\frac{240}{114.3}=2.1
$$

The degree of task similarity between $\operatorname{Task}_{V_{1}}$ and $\operatorname{Task}_{V_{2}}(s)$ is determined by using equation (3.5). The required parameters are calculated as follows:

$$
\begin{aligned}
& N\left\{\text { Task }_{V_{1}} \cap \text { Task }_{V_{2}}\right\}=2, \\
& N\left\{\text { Task }_{V_{1}}\right\}=6, \\
& N\left\{\text { Task }_{V_{2}}\right\}=4,
\end{aligned}
$$

which gives:

$$
s=\frac{2 \times N\left\{\text { Task }_{V_{1}} \cap \text { Task }_{V_{2}}\right\}}{N\left\{\text { Task }_{V_{1}}\right\}+N\left\{\text { Task }_{V_{2}}\right\}}=\frac{2 \times 2}{6+4}=0.4 .
$$

Therefore, the odds and probability of an optimal batch size transfer of $C^{*}=250$ to be required in order to minimize the performance measure (namely: average processing (service) time ( $A P T$ ) calculated over approximately 3000 jobs per station) given that the ratio of initial processing time to standard processing time of the task $(\rho)$ is 2.1 , the degree of task similarity $(s)$ is 0.4 , the worker forgetting rate $(F R)$ is $81 \%$, and the worker learning rate $(L R)$ is 


$$
\begin{aligned}
\log \left(\text { odds of } C^{*}=250\right) & =59.1698-1.8838(2.1)-6.5048(0.4) \\
& -42.5111(0.81)-18.0364(0.875)=2.40,
\end{aligned}
$$

the odds that the optimal batch transfer policy is $C^{*}=250$ is given by:

$$
\operatorname{odds}\left(C^{*}=250\right)=e^{2.40}=11.02,
$$

and the probability that the optimal batch transfer policy is $C^{*}=250$ is given by:

$$
P\left(C^{*}=250\right)=\frac{11.02}{1+11.02}=0.917 .
$$

Therefore, the probability of an optimal batch size of $C^{*}=250$ with these new

DRC shop conditions is significant at $91.7 \%$. Since this binary logistic regression equation has only two dependent variables: ' 0 ' and ' 1 ', corresponding to $C^{*}=10$ and $C^{*}=250$ respectively, the probability of an optimal batch transfer size of $C^{*}=250$ of $91.7 \%$ is equivalent to the probability of the optimal batch transfer size of $C^{*}=10$ being $8.3 \%(100 \%$ 91.7\%). In this case, given the DRC shop parameters specified in problem 1, it is evident that an optimal batch size of $C^{*}=250$ is more preferable than $C^{*}=10$.

Also, given the same parameters specified in the $C^{*}$ logistic regression equation, namely, the ratio of initial processing time to standard processing time of the task ( $\rho=2.1$ ), the degree of task similarity ( $s=0.4)$, the worker forgetting rate $(F R=81 \%)$, and the worker learning rate ( $L R=87.5 \%$ ), the odds and probability of an optimal upfront training policy batch size transfer of $F A P^{*}=5$ to be required in order to minimize the performance measure (namely: average processing (service) time ( $A P T$ ) calculated over approximately 3000 jobs per station) are calculated as follows:

$$
\begin{aligned}
\log \left(\text { odds of } F A P^{*}=5\right)= & 3.0171-1.5797(2.1)+1.5667(0.4) \\
& +6.5473(0.81)-9.7559(0.875)=-2.91
\end{aligned},
$$


and the probability that the optimal upfront training policy is $F A P^{*}=5$ is given by:

$$
P\left(F A P^{*}=5\right)=\frac{0.054}{1+0.054}=0.051
$$

Therefore, the probability of an optimal upfront training policy of $F A P^{*}=5$ is small at $5.1 \%$. Since the $F A P^{*}$ binary logistic regression equation also has only two dependent variables: ' 0 ' and '1', corresponding to $0 \leq F A P^{*} \leq 1$ and $F A P^{*}=5$ respectively, the probability of an optimal upfront training policy of $F A P^{*}=5$ of $5.1 \%$ is equivalent to the probability of the optimal upfront training policy of $0 \leq F A P^{*} \leq 1$ being $94.9 \%(100 \%-5.1 \%)$. As a result, given this information, the manager would suggest that the DRC shop should only provide either: no initial training or initial training up to the standard number of units $\left(0 \leq F A P^{*} \leq 1\right)$.

\subsection{Solution of DRC shop problem 2}

Again, before any calculations can begin, the new degree of task similarity parameter $S$ must be determined because a new Task $_{V_{3}}$ replaced the previous

Task $_{V_{2}}$. The required parameters are calculated as follows:

$$
\begin{aligned}
& N\left\{\text { Task }_{V_{1}} \cap \text { Task }_{V_{3}}\right\}=3, \\
& N\left\{\text { Task }_{V_{1}}\right\}=6, \\
& N\left\{\text { Task }_{V_{3}}\right\}=3,
\end{aligned}
$$

which gives,

$$
s=\frac{2 \times N\left\{\text { Task }_{V_{1}} \cap \operatorname{Task}_{V_{3}}\right\}}{N\left\{\text { Task }_{V_{1}}\right\}+N\left\{\text { Task }_{V_{3}}\right\}}=\frac{2 \times 3}{6+3}=0.67 .
$$


Also, the new initial processing time to standard processing time of the task $(\rho)$ is given by:

$$
\rho=\frac{y(1)}{y_{s}}=\frac{240}{43.6}=5.5 \text {. }
$$

Therefore the odds and probability of an optimal batch size transfer of $C^{*}=250$ to be required in order to minimize the performance measure if the new worker with a slower learning rate ( $L R=89 \%$ ) performed tasks that were more similar $s=0.67$ and had a larger initial processing time to standard time ratio $\rho=5.5$, are calculated as follows:

$$
\begin{aligned}
\log \left(\text { odds of } C^{*}\right. & =250)=59.1698-1.8838(5.5)-6.5048(0.67) \\
& -42.5111(0.81)-18.0364(0.89)=-6.04
\end{aligned}
$$

the odds that the optimal batch transfer policy is $C^{*}=250$ is given by:

$$
\operatorname{odds}\left(C^{*}=250\right)=e^{-6.04}=0.0024,
$$

and the probability that the optimal batch transfer policy is $C^{*}=250$ is given by:

$$
P\left(C^{*}=250\right)=\frac{0.0024}{1+0.0024}=0.0024
$$

Therefore, the probability of an optimal batch size of $C^{*}=250$ with these new DRC shop conditions is marginal at $0.24 \%$. Since this binary logistic regression equation has only two dependent variables: ' 0 ' and ' 1 ', corresponding to $C^{*}=10$ and $C^{*}=250$ respectively, the probability of an optimal batch transfer size of $C^{*}=250$ of $0.24 \%$ is equivalent to the probability of the optimal batch transfer size of $C^{*}=10$ being $99.76 \%(100 \%-0.24 \%)$. In this case, given a reduced rate of worker learning, different task characteristics, and a new standard time, it is evident that an optimal batch size of $C^{*}=10$ is now much more preferable than $C^{*}$ $=250$.

Again, using the same changes in DRC shop parameters as the new $C^{*}$ example, if a worker with a slower learning rate $(L R=89 \%)$ performed tasks that were more similar $(s=0.67)$ and had a larger initial processing time to standard time ratio $(\rho=5.5)$, the associated odds and probabilities are:

$$
\begin{aligned}
\log \left(\text { odds of } F A P^{*}=5\right)= & 3.0171-1.5797(5.5)+1.5667(0.67) \\
& +6.5473(0.81)-9.7559(0.89)=-8.0^{\prime}
\end{aligned}
$$


the odds that the optimal upfront training policy is $F A P^{*}=5$ is given by:

$$
\operatorname{odds}\left(F A P^{*}=5\right)=e^{-8.0}=0.0003
$$

and the probability that the optimal upfront training policy is $F A P^{*}=5$ is given by:

$$
P\left(F A P^{*}=5\right)=\frac{0.0003}{1+0.0003}=0.0003
$$

Therefore, the probability of an optimal upfront training policy of $F A P^{*}=5$ in with these new DRC shop conditions is even smaller at $0.03 \%$. This is equivalent to the probability of the optimal upfront training policy of $0 \leq F A P^{*} \leq 1$ being $99.97 \%(100 \%-0.03 \%)$. As a result, these new DRC shop conditions would also suggest to the manager that the DRC shop should only provide either: no initial training or initial training up to the standard number of units $\left(0 \leq F A P^{*} \leq 1\right)$. The summary of solutions for problem 1 and 2 of the DRC shop numerical example is given in Table 4.12 .

Table 4.12: Summary of results of numerical example for the binary logistic regression equation of model $C$

\begin{tabular}{|l|l|l|}
\hline Problem & $\begin{array}{l}\text { Musical instrument } \\
\text { DRC shop input } \\
\text { parameters }\end{array}$ & $\begin{array}{l}\text { Optimal policy } \\
\text { solution } \\
\text { parameters }\end{array}$ \\
\hline$\# 1$ & $\begin{array}{l}s=0.4, \rho=2.1, L R= \\
87.5 \%, F R=81 \%\end{array}$ & $C^{*}=250$ \\
$0 \leq F A P^{*} \leq 1$ \\
\hline$\# 2$ & $s=0.67, \rho=5.5, L R=$ & $C^{*}=10$ \\
& $89 \%, F R=81 \%$ & $0 \leq F A P^{*} \leq 1$ \\
\hline
\end{tabular}




\subsubsection{Implications of results for the five DRC issues}

The five possible issues that affect DRC shop performance measures as given by Hottenstein and Bowman (1998) are: (1) worker flexibility, (2) centralization of control, (3) worker assignment, (4) queue disciplines, and, (5) cost of transferring workers. The results of model $\mathrm{C}$ are obtained by examining the LFCM with a task similarity factor, and as a result, the results of model $\mathrm{C}$ regarding the shop performance measures of Hottenstein and Bowman (1998) are identical to those obtained by the results of model B.

\subsubsection{Model $C$ conclusions}

Model $\mathrm{C}$ resulted in two unexpected findings: (1) $C^{*}$ decreases as the learning rate slows between a learning rate of $70 \%$ and $80 \%$, and increases again to a maximum at a learning rate of approximately $85 \%$ before decreasing again at a learning rate of $90 \%$ (see Figure 4.4 ), and, (2) the distribution of the 625 output variables for both the optimal batch transfer size policy $\left(C^{*}\right)$ and the optimal level of upfront training policy $\left(F A P^{*}\right)$ are irregular with few or no data points in the middle sections of the specified ranges. The optimal values seem to cluster at the extremes of both specified ranges of $C$ and $F A P$.

The unexpected finding in (1) that suggests that $C^{*}$ tends to decrease with respect to the learning rate is contrary to intuition because it can be assumed that, as learning deteriorates, batch sizes should increase to compensate for the large forgetting losses. This observation was due to the unique factor interactions that resulted in optimized performance measures that were not observed by just examining the performance behaviour at one factor setting or by averaging across factors, such as in Figure 4.4. Also, the expected rise in the behaviour of $C^{*}$ at a learning rate of approximately $85 \%$ (see Figure 4.4 ) was due to the interaction between the effect of the batch transfer size and the initial training batch size. Namely, if the worker has a fast learning rate, he/she only initially produces the batch transfer size number of units $(C)$ because this quantity is greater than the number of units needed to reach the standard time of the worker $\left(n_{s}\right)$. However, if the learning rate of the worker is slower, $n_{s}$ increases and exceeds the batch transfer size. Since the worker under an $F A P-1$ or an $F A P-2$ upfront training policy must remain at the first station until $n_{s}$ units or $2 n_{s}$ units are processed, he/she gains enough experience as to cause the overall optimal policy to increase beyond that of even a worker with a 
faster learning rate that had less initial training. However, this benefit decreases as the detrimental effect of a low learning rate outweighs the benefit of producing a large initial training batch. This effect explains the unexpected rise the behaviour of $C^{*}$ found in Figure 4.4. The learning rate at which this rise occurs was shown to be dependent on the value of $\rho$; and in model $\mathrm{C}$, the average influence over the range of $\rho$ caused this overall rise to occur at an approximate worker learning rate of $85 \%$.

The unexpected finding (2) was circumvented by the use of binary logistic multiple regression that necessitated excluding a total of 52 data points located in the middle sections of the distributions of $C^{*}$ and $F A P^{*}$. Therefore, only $91.7 \%$ (573/625) of the total possible DRC situations as specified in Table 3.5 were accounted for by using this regression technique. This compromise was deemed as the preferred trade-off because the alternative of including all the data would result in either erroneous or irrelevant results. Consequently, due to the distribution of the output variables, the functions in (3.10) and (3.11) were fitted to the values using binary logistic multiple-regression. Model C provided a pseudo DRC shop situation and demonstrated the application of the logistic regression equations with a numerical example. The development of these equations may be important from a managerial perspective because they suggest preferred settings for controllable DRC shop factors (training and transfer policies) given existing factors that cannot be as easily changed (job similarity, task-times, and worker learningforgetting rate).

\subsection{Chapter 4 summary}

The previous chapter 4 has provided the results, analysis and conclusions to the models in this thesis and has discussed how they compare to those of Jaber et al. (2003) and the five possible issues that affect DRC shop performance given by Hottenstein and Bowman (1998). Chapter 5 provides thesis conclusions and suggestions for further work in this area. 


\section{Chapter 5: thesis conclusions and recommendations for further research}

\subsection{Thesis summary}

This thesis has extended upon Jaber et al. (2003) that investigated worker learning and forgetting phenomenon in dual resource constrained system (DRC) settings. This thesis also introduced the concept of worker learning and forgetting in a two and three stage DRC system according to the dual-phase learning-forgetting model (DPLFM) developed by Jaber and Kher (2002). This learning and forgetting model is based on the theory that a task has separate cognitive and motor requirements. The effect of including task similarity was also examined. The experiments in model A, model $\mathrm{B}$, and model $\mathrm{C}$ consisted of deterministic simulation models; the results were compared to those of Jaber et al. (2003) and the five possible issues that affect DRC shop performance given by Hottenstein and Bowman (1998).

Chapter 1 provided a survey of research done in the areas of DRC systems, worker learning theory, learning and forgetting models, and the learning and forgetting phenomenon in DRC systems. Chapter 2 summarized the development of the dual-phase learning-forgetting model (DPLFM), the learning and forgetting model used in model A and model B of this thesis. Chapter 3 discussed the modeling or experimental design used in model A, model B, and model $\mathrm{C}$ of this thesis. Chapter 4 provided the results, analysis and conclusions to these models and discussed how they compare to those of Jaber et al. (2003) and the five possible issues that affect DRC shop performance given by Hottenstein and Bowman (1998). This thesis ended at model C because the applicability of model $\mathrm{C}$ would have to be further tested before the inclusion of the DPLFM (for example) as a possible extension of model C. Also, the author wished to keep the scope of the thesis within the boundaries of worker flexibility issues; whereas to also include issues such as machine flexibility (group technology (GT)) would possibly require additional collaboration with persons familiar with such an established research area. These topics are left as possible extensions and are discussed in section 5.4. The remainder of this chapter summarizes the unique contributions provided by this thesis, exposes limitations in the investigation of this thesis, and proposes possible future research extensions. 


\subsection{Thesis contributions to DRC research}

This thesis has provided several contributions to DRC research. Firstly, in model C, it has developed binary logistic equations that may be important from a managerial perspective because they express preferred settings for controllable DRC shop factors such as worker training and transfer policies as functions of existing factors that cannot be as easily changed such as the degree of job similarity, task processing times, and the learning and forgetting rate of the worker. A pseudo DRC shop situation demonstrated the application of these logistic regression equations with a numerical example. This technique may enhance future DRC research by recognizing the need for research pertaining to job shops that are already in operation and that are either resistant to change or incapable of significant change.

Secondly, in model A, it has shown that the type of task that the worker performs with respect to its learning rate and proportion of cognitive and motor elements is a factor that could be included in future DRC research. The DPLFM developed by Jaber and Kher (2002) is a model that can provide a measure that recognizes both these inherent task characteristics. Thirdly, in model $B$, the results have suggested that the DPLFM emphasized a greater benefit for upfront training and more a frequent worker transfer policy than the LFCM when tasks are similar, and supported the conclusions of Jaber et al. (2003) by an even greater extent that it is possible to use more flexibility (up to three tasks learned) in DRC shops with similar tasks. Furthermore, this thesis has also developed a DRC summary matrix that could be used as a guide for future DRC research.

\subsection{Thesis limitations}

The thesis investigation has two important limitations. The first limitation is that this research area has an absence of studies that provide a quantitative unit to measure the benefits of added DRC shop flexibility. As previously mentioned, DRC shops have provided benefits such as the reduction of manufacturing lead times (reduction in WIP), improved customer service, and the ability to adapt to frequent changes in product demand (Kher, 2000). However, in order to truly weigh the benefits of DRC shop flexibility against the detriments of DRC shop forgetting losses, a significant trade-off analysis should also utilize a single quantitative unit to measure the benefits of flexibility. Intuitively, in order for a significant trade-off analysis to be conducted, this unit should be similar to the unit used to measure the detriments of flexibility. 
The second limitation is the performance measure used in this thesis, by Jaber et al. (2003), and by Kher et al. (1999) of average processing (service) time ( $A P T$ ) calculated over approximately 3000 jobs per station. Modern DRC shop products may have product life-cycles much lower than a total production count of 3000 jobs per station (Vokurka, and Lummus, 2003). For example, from the work experience of the author, typical yearly production counts of make-to-order shop products such as customized fine furniture average approximately 300 units with approximately 225 different units within that group. Approximately $50 \%$ of the sub-tasks are similar between products. Therefore, on average, only $188[(300-225)+(0.5) \times 225]$ units are processed per year with an average life-cycle of seven years per product. Consequently, the average life-cycle production count for a customized fine furniture shop is approximately 1316 mits. The graph in Figure 5.1 illustrates the average processing (service) time ( $A P T$ ) versus the centralization of control policy $C$ (the batch transfer quantity) for various levels of product life-cycles measured in units per station $(J)$ for the LFCM. Note that when the product life-cycles are $J=3000,2500,2000$ units per station the optimal batch transfer quantity is 250 units $\left(C^{*}=250\right)$. However, for items with shorter product life-cycles such as fine furniture ( $J=1500,1000)$ units per station), items characteristic of a DRC shop, the optimal batch transfer quantity is 10 units $\left(C^{*}=10\right)$.

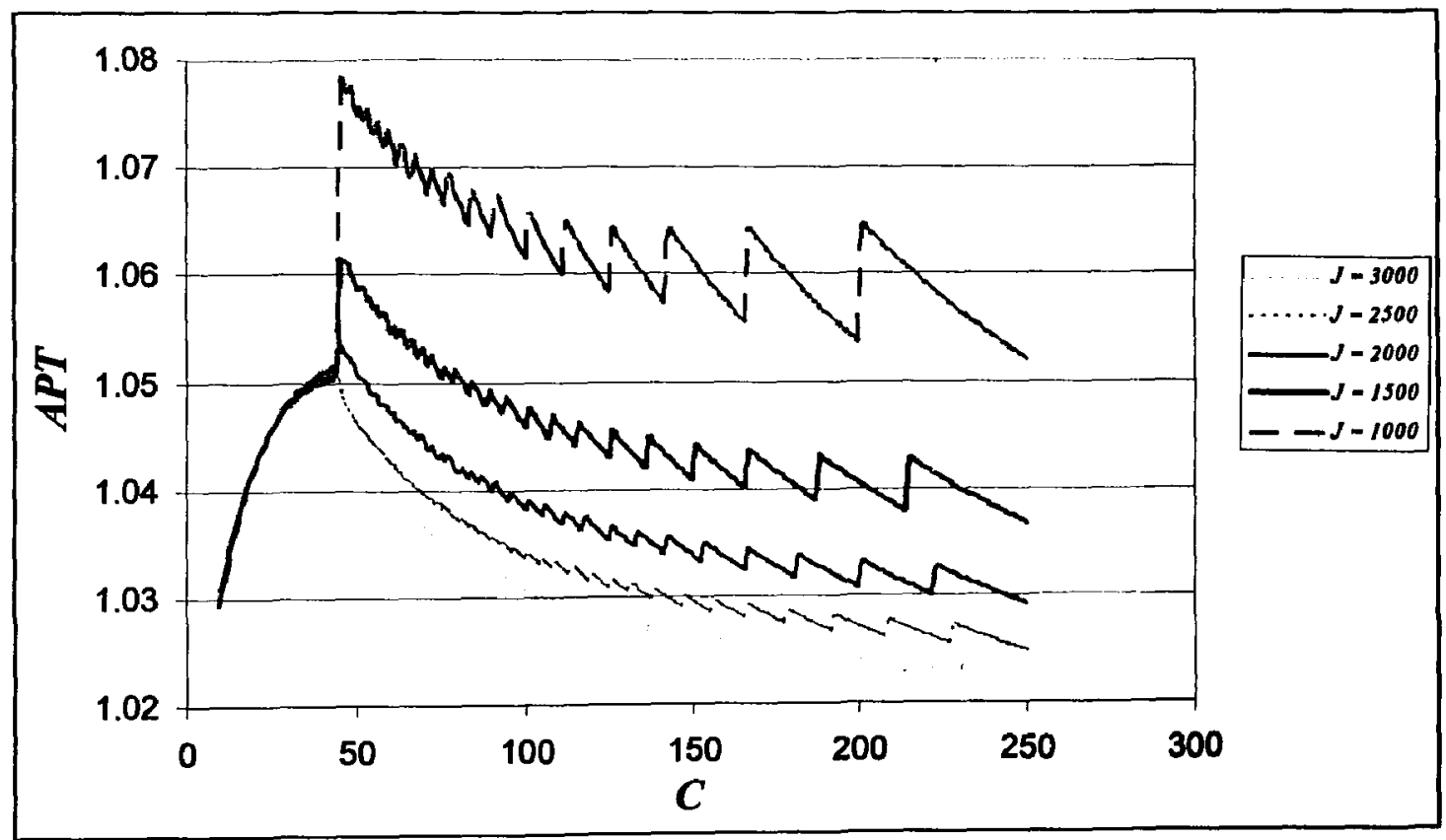

Figure 5.1: Plot of $A P T$ versus $C$ for various levels of $J$ ( $s=0, N=2$, $\rho=7, L R=70 \%, F R=80 \%$, LFCM) 
Consequently, the performance measure used in this thesis, by Jaber et al. (2003), and Kher et al. (1999) of average processing (service) time ( $A P T$ ) calculated over approximately 3000 units per station ( $A P T$ ) may not be the most preferable DRC shop performance measure since DRC shop products may have shorter life-cycles than 3000 units per station (i.e. 1316 units). However, for consistency of comparison with Jaber et al. (2003) and Kher et al. (1999), 3000 units per station was retained as the duration of the production simulation study.

\subsection{Recommendations for further research}

The following sections outline some possible research extensions that might be of some interest.

\subsubsection{Combine DRC and GT}

The benefits and drawbacks of worker learning and forgetting could be integrated with ongoing research on the combined effect of using worker flexibility (DRC systems) in shops with machine flexibility (commonly referred to as group technology (GT)). For interested readers, see Jensen (2000).

\subsubsection{Equations using DPLFM}

A possible extension to model $\mathrm{C}$ of this thesis may include developing similar equations using the DPLFM as the learning forgetting model instead of the LFCM. In this case the new equations could take the form:

$$
g(s, N, \rho, T, F R, R)=\left(C^{*}, F A P^{*}\right)
$$

where $T$, and $R$ represent the additional DPLFM task parameters of task-type and the ratio of time for the first unit under purely cognitive conditions to time for the first unit under purely motor conditions respectively. Depending on the distribution of the output data, a binary logistic regression equation may not be appropriate for equation (5.1). Multi-factor cubic spline interpolation may be a more feasible curve fitting technique if the data results are not clustered 
at the extremes of both specified ranges of $C$ and $F A P$ (as shown in Figure 4.24 and Figure 4.25) but are more equally spread across the specified range of the dependent variables.

\subsubsection{Task sequence}

The examination of the effect of task sequence on forgetting losses could also be studied. For example, in a $N=3$ DRC shop, the worker can transfer from a cognitive task to a motor task and then back to a cognitive task, i.e. $\left(T_{C}-T_{M}-T_{C}\right)$, or from a motor task to a median task and then back to a cognitive task, i.e. $\left(T_{M}-T_{M e d}-T_{C}\right)$, etc. Depending on the sequence of task-type assignments the optimal sequence can be determined in order to minimize forgetting losses.

\subsubsection{Worker attrition rates}

The periodic loss of trained workers for various reasons presents a serious problem to the performance of DRC shops. This is because newly hired workers have to undergo more upfront training than experienced workers. As a result, the inclusion of the rate at which workers leave (equal to the rate at which new workers are acquired), also called the worker attrition rate, is an important factor that could be included as a possible research extension. Kher et al. (1999) included an attrition rate that was measured as the ratio between the worker attrition interval and the length of the initial training period (atr/tr). This particular method of measuring worker attrition is an improvement over a simple yearly attrition rate (as used by earlier studies) because it better represents the detrimental effect of each worker attrition occurrence by relating it to the length of the subsequent training period (Kher et al., 1999). 


\subsubsection{Further recommendations - DRC summary matrix}

\subsubsection{DRC summary matrix overview}

As shown in the appendix (see section 7.1), the DRC summary matrix is an organized table listing DRC research criteria amongst the titles of the leftmost column (heading for rows) of the matrix and listing DRC research studies along the upper row (heading for columns). In section 7.1.1 the model assumption issues 'Workload issues' and 'Operating issues' are listed in the leftmost column of the table. They are the headings for the rows they contain. The column headings 'Jaber et al. (2003)', 'Thesis - model A', 'Thesis - model B', and 'Thesis - model C' are the column headings for the specified DRC research studies. The experimental factors of each study are listed in bold font text and the fixed factors are listed in regular font. For example, in section 7.1.1, for the Workload issue 'Mean job service rate', an initial and standard processing time of four units and one unit respectively are specified as fixed factors (regular font text) for the DRC study Jaber et al. (2003). However, for the DRC study Thesis - model C, the mean job service rate is actually an experimental factor (bold font text) that has five different settings for both the initial and standard processing times.

The partition of the DRC matrix illustrated in section 7.1.1 denotes the model assumptions of each study listed in every column, whereas the partition illustrated in section 7.1.2 denotes the model results and conclusions of each study as they relate to DRC shop performance measures categorized by the particular experimental factors examined.

This DRC summary matrix could be used as a guide for future research by just adding subsequent research (or newly discovered old or new research) as new columns in the matrix. This could aid the research path by indicating the possible performance measures or experimental factors overlooked in current research and/or avoiding redundant research tangents by clearly listing the conclusions of previous studies.

The following section uses the DRC summary matrix as a guide for recommending further DRC research. 


\subsubsection{Further recommendations for DRC research}

The DRC summary matrix could be also used as a guide for recommending future research initiatives, for example: in the design issues section pertaining to worker learning and forgetting of the summary matrix (see section 7.1.1), one row mentions a possible situation where the performance of the worker either temporarily remains constant or increases shortly after an interruption. Since the three studies listed so far in the summary matrix assume that worker forgetting begins (performance decreases) as soon as an interruption occurs, a natural inquiry would be to examine worker forgetting experimentally to see if this occurrence was observed.

Also, in the design issues section pertaining to worker product variety (as categorized by McKreery and Krajewski, (1999)) of the summary matrix (see section 7.1.1), one row mentions a possible situation where there is product turnover measured as a rate per year. The three studies listed so far in the summary matrix assume no product turnover. However, a further study could include a rate of product turnover to compensate for the aforementioned limitation in section 5.3 regarding the long production simulation duration of 3000 units per station. The inclusion of a product turnover rate may emulate a model containing a shorter product life-cycle more characteristic of make-to-order products commonly found in dual resource constrained shops. 


\section{REFERENCES}

Alternburg, K., D. Griscom, J. Hart, F. Smith, G. Wohler. (1999). Just-in-time logistics support for the automobile industry. Production and Inventory Management Journal, 40(2), 59-66.

Anderlohre, G. (1969). What production breaks cost. Industrial Engineering, Sept., 34-36.

Argote, L., Epple, D., (1990). Learning Curves in Manufacturing. Science, 247, 920-924.

Arzi, Y. and Shtub (1997). Learning and forgetting in mental and mechanical tasks: a comparative study. IIE Transactions, 29, 759-768.

Ash, R., Smith-Daniels, D.E., (1999). The Effects of Learning, Forgetting, and Relearning on Decision Rule Performance in Multiproject Scheduling. Decision Sciences, 30(1), 47-82.

Bailey, C.D., (1989) Forgetting and the learning curve: A laboratory study. Management Science, 35(3), 340-352.

Baloff, N. (1971). Extensions of the learning curve - some empirical results, Operational Research Quarterly, 22(4), 329-305.

Belkaoui, A., (1986). The Learning Curve, Quorum Books, Westport, CT.

Bobrowski, P.M., and Park, P.S. (1993). An evaluation of labor assignment rules when workers are not perfectly interchangeable. Journal of Operations Management, 3, 257-268.

Bohlen, G.A. and Baraney, J.W. (1976). A learning curve prediction model for operators performing industrial bench assembly operations. International Journal of Production Research, 14(2), 295-303.

Buck, J.R., Tanchoco, J.M.A. and Sweet, A.L. (1976). Parameter estimation methods for discrete exponential learning curves, AIIE Transactions., 25(6), 34-47.

Carlson, J.C., Rowe, A.J., (1976). How much does forgetting cost? Industrial Engineering, $8,40-47$.

Chapra, S.C., R. P. Canale (2002). Numerical Methods for Engineers, Fourth Ed. New York: McGraw-Hill Companies Inc. 
Cody, R.P., Smith J.K (1997). Applied Statistics and the SAS Programming Language, Fourth Ed. New Jersey: Prentice Hall Inc.

Dar-El, E.M., (2000). Human Learning: From Learning Curves to Learning Organizations, Kluwer Academic Publishers, Norwell Massachusetts 02061.

Dar-El, E.M., K. Ayas and I. Gilad, (1995). A dual phase model for the individual learning process in industrial tasks, IIE Transactions, 27, 265-271.

De Jong, J.R., (1957). The effect of increased skills on cycle time and its consequences for time standards. Ergonomics, 1, 51-61.

Fryer, J.S. (1973). Operating policies in multi-echelon dual-constraint job shops. Management Science, 9, 1001-1012.

Fryer, J.S. (1976). Organizational segmentation and labor transfer policies in labor and machine limited production systems. Decision Science, 7, 725-738.

Globerson, S., Levin, N., Shtub, A., (1989). The impact of breaks on forgetting when performing a repetitive task. IIE Transactions, 21(4), 376-381.

Globerson, S., Nahumi, A., \& Ellis, S. (1998). Rate of forgetting for motor and cognitive tasks. International Journal of Cognitive Ergonomics, 2(3), 181-191.

Gunther, R.E. (1979). Server transfer delays in a dual resource constrained parallel queuing system. Management Science, 12, 1245-1257.

Hewitt, D., Sprague, K., Yearout, R., Lisnerski, D., Sparks, C., (1992). The effects of unequal relearning rates on estimating forgetting parameters associated with performance curves. International Journal of Industrial Ergonomics, 10, 217-224.

Hottenstein, Michael P., Bowman, Sherry A., (1998). Cross training and worker flexibility: a review of DRC system research. Journal of High Technology Management Research, 9(5), 157174. 
Jaber, M.Y., (1996). The effect of learning and forgetting on the economic manufacture quantity. PhD Thesis. University of Nottingham, U.K.

Jaber, M.Y. and Bonney, M.C., (1996). Production Breaks and the Learning curve: The forgetting phenomena. Applied Mathematical Modelling, 20, 162-169.

Jaber, M.Y. and Bonney, M.C., (1997). A comparative study of learning curves with forgetting. Applied Mathematical Modelling, 21, 523-531.

Jaber, M.Y. and Bonney, M.C., (1998). The effects of learning and forgetting on the optimal lot size quantity of intermittent production runs. Production Planning and Control, 9(1), 20-27.

Jaber, M.Y. and Kher, H.V., (2002). The dual phase learning-forgetting model. Int. $J$. Production Economics, 76(3), 229-242.

Jaber, M.Y. and Kher, H.V., Davis, D.J., (2003). Countering forgetting through training and deployment. International Journal of Production Economics, 85(1), 33-40.

Jensen, J.B., (2000). The impact of resource flexibility and staffing decisions on cellular and departmental shop performance. European Journal of Operational Research, 127(2), 279-296.

Karnes, C.L., L.P. Karnes, (2000). Ross controls: A case study in mass customization. Production and Inventory Management Journal, 41(3), 1-4.

Kher, H.V., Malhotra, M.K., Philipoom, P.R., Fry, T.D., (1999). Modeling simultaneous worker learning and forgetting in dual constrained systems. European Journal of Operational Research, 115, 158-172.

Kher, H.V., (2000). Examination of flexibility acquisition policies in dual resource constrained job shops with simultaneous worker learning and forgetting effects. Journal of the Operational Research Society, 51, 1-10.

Knecht, G.R. (1974). Costing, technical growth and generalized learning curves, Operational Research Quarterly, 25(3), 487-491.

Konz, S., (1990). Work Design: Industrial Ergonomics. Third Edition. Publishing Horizons, Inc., Worthington, Ohio, 423-439. 
Lippert, S., (1976). Accounting for prior practice in skill acquisition studies, International Journal of Production Research, 14(2), 285-293.

Malhotra, M.K., Fry, T.D., Kher, H.V., Donohue, J.M., (1993). The Impact of learning and labour attrition on worker flexibility in dual resource constrained job shops. Decision Sciences, 24(3), 641-663.

McKenna, K., Glendon, S.P., \& Glendon, A. I., (1985). Occupational first aid training: Decay in cardiopulmonary resuscitation skills. Journal of Occupational Psychology, 58, 109-117.

McKreery, J.K, Krajewski, L.J., (1999). Improving performance using workforce flexibility in an assembly environment with learning and forgetting effects. International Journal of Production Research, 37(9), 2031-2058.

Montgomery, D.C., (1997). Design and Analysis of Experiments (Fourth Edition). John Wiley \& Sons, Inc.

Naitove, M., (2003). Editorial: Working harder, working smarter. Plastics Technology, 49(1), 9.

Nelson, R.T., (1967). Labor and Machine Limited Production Systems. Management Science, 13(9), 648-671.

Park, P.S. and Bobrowski, P.M., (1989). Job release and labor flexibility in a dual resource constrained job shop. Journal of Operations Management, 8(3), 230-249.

Pepinsky, P.N. and Pepinsky, H.B., (1960). The effects of task complexity and time pressure upon team productivity. Journal of Applied Psychology, 44(1), 34-39.

SAS Online Doc. ${ }^{\circledR}$ version 8, Copyright (C 1999, SAS institute Inc., Cary, NC,USA. All rights reserved, (http:/v8doc.sas.com/sashtml/), [accessed January 20, 2004].

Shafer, M. S., Nembhard, D.A., Uzumeri, M.V., (2001). The effects of Worker Learning, Forgetting, and Heterogeneity on Assembly Line Productivity. Management Science, 47(12), 1639-1653. 
Silver, E. A. et al., (1998). Inventory Management and Production Planning and Scheduling. New York: John Wiley \& Sons, Inc.

Smith, Jason, (1989). Learning Curve for Cost Control, Industrial Engineering and Management Press, Norcross, GA, 1989.

Sparks, C., and Yearout, R., (1990). The impacts of visual display units for highly cognitive tasks of learning curve models. Computers and Industrial Engineering, 19(1- 4): 351-355.

Steedman, I. (1970). Some improvement curve theory. International Journal of Production Research, 8(3), 189-205.

Sule, D.R., (1978). The effect of alternate periods of learning and forgetting on economic manufacturing quantity. AIIE Transactions, 10(3), 338-343.

Teplitz, C.J., (1991). The Learning Curve Deskbook, Quorum Books, New York.

Tompkins, J.A., (2002). New rules emerging in manufacturing. Plant Engineering, 56(3), 26-28.

Treleven, M.D., (1987). The timing of labour transfers in dual resource-constrained systems: 'push' vs. 'pull' rules. Decision Sciences, 18, 73-88.

Treleven, M.D., (1988). A comparison of flow and queue time variances in machine-limited versus dual-resource-constrained systems. IIE Transactions, 20(1), 63-67.

Treleven, M.D., (1989). A review of the dual resource constrained system research. IIE Transactions, 21(3), 279-287.

Vokurka, R.J., R.R. Lummus, (2003). Better supply chains with Baldrige. Quality Progress, 35(4), 51-57.

Wisner, J.D., J.N. Pearson, (1993). An exploratory study of the effects of operator relearning in a dual resource constrained job shop. Production and Operations Management Journal, 2(1), 55-68.

Wood, R.E., (1986). Task Complexity: definition of the construct. Organizational Behaviour and Human Decision Processes, 37, 60-82.

Wright, T., (1936) Factors affecting the cost of airplanes. Journal of Aeronautical Science, 3(4), 122-128. 


\subsection{DRC summary matrix}

\subsubsection{Model assumptions}

\begin{tabular}{|c|c|c|c|c|c|}
\hline & & \multirow[b]{2}{*}{ Jaber et al. (2003) } & & \\
\hline & & & $\begin{array}{c}\text { Thesis - model } \\
\text { A }\end{array}$ & $\begin{array}{c}\text { Thesis - model } \\
\text { B }\end{array}$ & $\begin{array}{l}\text { Thesis - model } \\
\text { C }\end{array}$ \\
\hline $\begin{array}{l}\text { Type of } \\
\text { Issue }\end{array}$ & Issue Description & Modeling Method & $\begin{array}{l}\text { Modeling } \\
\text { Method }\end{array}$ & $\begin{array}{l}\text { Modeling } \\
\text { Method }\end{array}$ & $\begin{array}{l}\text { Modeling } \\
\text { Method }\end{array}$ \\
\hline \multirow{12}{*}{ 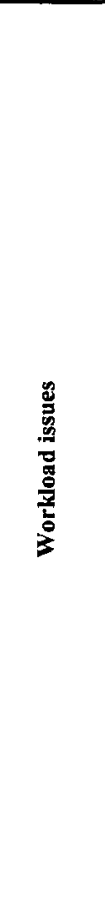 } & Deterministic job a rrivals? & Yes & " " & " " & $7 "$ \\
\hline & Probabilistic job arrivals? & No & " " & " " & " " \\
\hline & Job arrival density function $(a)$ & $\begin{array}{l}\text { N/A since arrivals are } \\
\text { deterministic }\end{array}$ & " " & " " & $\overline{\prime \prime \prime}$ \\
\hline & Mean job arrival rate(s) (ㄱ) & $\begin{array}{l}\text { Equal to service rate i.e. } \\
\text { constant availability of work } \\
\text { with no queue formation }\end{array}$ & $" "$ & " " & " " \\
\hline & Deterministic job service rate? & Yes & $"$ " & " " & $"$ \\
\hline & Probabilistic job service rate? & No & " " & $"$ & $" 1$ \\
\hline & Job service density function $\left(\chi_{i}\right)$ & $\mathrm{N} / \mathrm{A}$ & " " & $"$ & " " \\
\hline & Mean job service rate(s) $\left(\gamma_{i}\right)$ & $\begin{array}{l}\text { A } y(l) \text { and } y_{s} \text { of } 4.0 \text { and } 1.0 \\
\text { time units per job respectively } \\
\text { is specified }(\rho=4) \text {. }\end{array}$ & $" 1$ & $"$ " & $\begin{array}{l}y(l)=2,3,4, \\
5,6 \text { and } \\
y_{2}=1 \\
\text { corresponding } \\
\text { to five } \\
\text { different } p \\
\text { values: } \\
p=2,3,4,5,6\end{array}$ \\
\hline & $\begin{array}{l}\text { Is the system notionally balanced? } \\
\text { (average service time of the average } \\
\text { worker identical across stations) }\end{array}$ & $\begin{array}{l}\text { Only one worker, therefore } \\
\text { N/A. }\end{array}$ & " " & 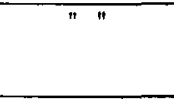 & " " \\
\hline & Deterministic job routing? & Yes & " " & $" n$ & $"$ \\
\hline & Probabilistic job routing? & No & $4 "$ & $"$ & $"$ \\
\hline & Probability matrix from stage $i$ to $j\left(p_{i j}\right)$ & $\mathrm{N} / \mathrm{A}$ & $"$ " & $" \overline{1 "}$ & " " \\
\hline \multirow{4}{*}{ 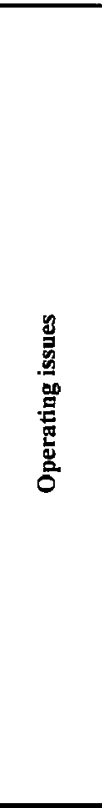 } & $\begin{array}{l}\text { Is the job release policy a finite or an } \\
\text { infinite loading policy? If finite, what rule } \\
\text { was used? }\end{array}$ & $\begin{array}{l}\text { A finite capacity policy with a } \\
\text { rule that a job is released only } \\
\text { after the worker has completed } \\
\text { processing the previous job. }\end{array}$ & " " & " " & " " \\
\hline & $\begin{array}{l}\text { Global, local, or dynamic queuc } \\
\text { disclipline(s)? }\left(q_{i}\right)\end{array}$ & $\begin{array}{l}\text { No queue formed, therefore } \\
\text { N/A. }\end{array}$ & " " & $"$ & " " \\
\hline & $\begin{array}{l}\text { Centralization of control ('when' rule) }\left(\pi_{i}\right) \\
\text { (e.g.: fixed/floating) } \\
\text {-workers that only left an assigned 'home } \\
\text { station' after the queue was empty and that } \\
\text { returned to the home station after the work } \\
\text { was completed (fixed) } \\
\text {-workers that move to another station they } \\
\text { are additionally trained for with the longest } \\
\text { queue affer the completion of every unit } \\
\text { (floating). } \\
\text { (see McCreery and Krajewski ,1999) }\end{array}$ & $\begin{array}{l}\text { Neither fixed nor floating, the } \\
\text { worker moves from the } \\
\text { station a fter a fixed number } \\
\text { of jobs a re completed, } \\
\text { e.g.:batch size of } 10,25 \text {, or } \\
250 \text {. }\end{array}$ & $"$ & $"$ & $\begin{array}{l}\text { In this model, } \\
\text { the } \\
\text { centralization } \\
\text { of control rule } \\
\text { was an } \\
\text { opttmized } \\
\text { output factor } \\
\text { that was } \\
\text { searched } \\
\text { within the } \\
\text { range } 10 \text { - } 250 \\
\text { units in } \\
\text { increments of } \\
\text { one unit. }\end{array}$ \\
\hline & Worker assignment rule ('where' rule) ( $l)$ & $\begin{array}{l}\text { The worker is always } \\
\text { transferred to the next stage, } \\
\text { namely, to } 1 \text { or } 2 \text { in the } 2 \text { stage } \\
\text { model, or to } 1,2 \text {, or } 3 \text { in the } 3 \\
\text { stage model. }\end{array}$ & " " & " " & $n n$ \\
\hline
\end{tabular}




\begin{tabular}{|c|c|c|c|c|c|}
\hline & & Jaber et al. (2003) & $\begin{array}{c}\text { Thesis - model } \\
\text { A }\end{array}$ & $\begin{array}{c}\text { Thesis - model } \\
\text { B }\end{array}$ & $\begin{array}{l}\text { Th } \\
\text { mo }\end{array}$ \\
\hline $\begin{array}{l}\text { Type of } \\
\text { Issue }\end{array}$ & Issue Description & Modeling Method & $\begin{array}{l}\text { Modeling } \\
\text { Method }\end{array}$ & $\begin{array}{l}\text { Modeling } \\
\text { Method }\end{array}$ & $\begin{array}{r}\text { Mo } \\
\text { M6 }\end{array}$ \\
\hline \multirow{17}{*}{ 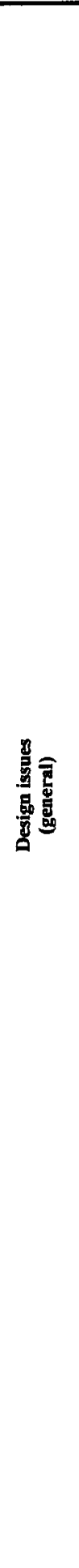 } & $\begin{array}{l}\text { Number of stages in the system } \\
\text { (m) }\end{array}$ & Modeled as 2 and 3 stages. & $" 1$ & $n$ & 7 \\
\hline & $\begin{array}{l}\text { Number of identical machines at } \\
\text { each stage } i\left(c_{i}\right)\end{array}$ & One machine per stage. & " " & $n$ & $\bar{"}$ \\
\hline & $\begin{array}{l}\text { How many different tasks can } \\
\text { each machine perform? } \\
\text { (i.e. is there machine flexibility or } \\
\text { group technology (GT)) }\end{array}$ & $\begin{array}{l}\text { Each machine is assumed to } \\
\text { process only one task, therefore } \\
\text { no machine flexibility is } \\
\text { assumed. }\end{array}$ & $"$ & " & " \\
\hline & $\begin{array}{l}\text { The number of workers in the } \\
\text { system (w) }\end{array}$ & 1 & " " & $n$ & $\overline{\prime \prime}$ \\
\hline & $\begin{array}{l}\text { What are the labour utilization } \\
\text { rate(s)? }\end{array}$ & $100 \%$ & $" \quad "$ & " $"$ & $\overline{\prime \prime}$ \\
\hline & $\begin{array}{l}\text { What is the relative efficiency of } \\
\text { worker } j \text { on stage } i\left(e_{j i}\right)\end{array}$ & $\begin{array}{l}\text { Only one worker, therefore } \\
\text { N/A. }\end{array}$ & $" 1$ & $n "$ & 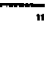 \\
\hline & Can the jobs revisit stations? & $\begin{array}{l}\text { Yes, the worker starts back at } \\
\text { the first station after completing } \\
\text { a cycle. }\end{array}$ & $" n$ & " n & $"$ \\
\hline & Is job pre-emption allowed? & No. & " " & $n$ & $\bar{"}$ \\
\hline & $\begin{array}{l}\text { Static or dynamic scheduling of } \\
\text { jobs? }\end{array}$ & $\begin{array}{l}\text { There are no arrivals until a } \\
\text { unit is complete; therefore it is } \\
\text { a simple static, first-come-first- } \\
\text { serve model. }\end{array}$ & " " & $7 "$ & $\bar{n}$ \\
\hline & $\begin{array}{l}\text { Is there a job transfer time } \\
\text { between stations? }\end{array}$ & No. & " " & $" 1$ & $\bar{"}$ \\
\hline & $\begin{array}{l}\text { Is there worker attrition rates, if } \\
\text { so, what are they? }\end{array}$ & No. & " " & $n$ & $"$ \\
\hline & Is there job blocking? & $\begin{array}{l}\text { Since there is only one task in } \\
\text { progress at any one time, this is } \\
\text { N/A. }\end{array}$ & " " & $\pi n$ & $\bar{~}$ \\
\hline & $\begin{array}{l}\text { Is there a distinction made } \\
\text { between procedural and } \\
\text { continuous tasks (Globerson et } \\
\text { al.,1989), if so, how is this } \\
\text { distinction modeled? }\end{array}$ & No. & " " & $" n$ & $\bar{n}$ \\
\hline & $\begin{array}{l}\text { What is/are the performance } \\
\text { measure(s) of the system? }\end{array}$ & $\begin{array}{l}\text { Average processing (service) } \\
\text { time calculated over } \\
\text { approximately } 3000 \text { jobs per } \\
\text { station. Also, final efficiency } \\
\text { defined as the processing time } \\
\text { of the first job in cycle } n+1 \text {, } \\
\text { where } n \text { is the number of cycles } \\
\text { of data collection in the } \\
\text { simulation run. }\end{array}$ & $\begin{array}{l}\text { Average } \\
\text { processing } \\
\text { (service) time } \\
\text { calculated over } \\
\text { approximately } \\
3000 \text { jobs per } \\
\text { station. }\end{array}$ & " " & $\bar{"}$ \\
\hline & $\begin{array}{l}\text { Are there setup times on some } \\
\text { operations? Are they sequence } \\
\text { dependent? }\end{array}$ & No setup time assumed. & " " & $" 1$ & 7 \\
\hline & Is there a cycle length specified? & $\begin{array}{l}\text { Yes, a cycle is complete when } \\
\text { a worker visits } 1 \text { and } 2 \text { stations } \\
\text { when he/she is trained for } 2 \\
\text { stations, and when a worker } \\
\text { visits } 1,2 \text {, and } 3 \text { stations when } \\
\text { he/she is trained for } 3 \text { stations }\end{array}$ & " " & $n n$ & $\overline{7}$ \\
\hline & $\begin{array}{l}\text { Are the products perishable? } \\
\text { (i.e.: a non-infinite storage } \\
\text { assumption, also see complexity } \\
\text { below) }\end{array}$ & $\begin{array}{l}\text { The product is assumed to have } \\
\text { an infinite storage life. }\end{array}$ & " " & $n$ & $\bar{n}$ \\
\hline
\end{tabular}




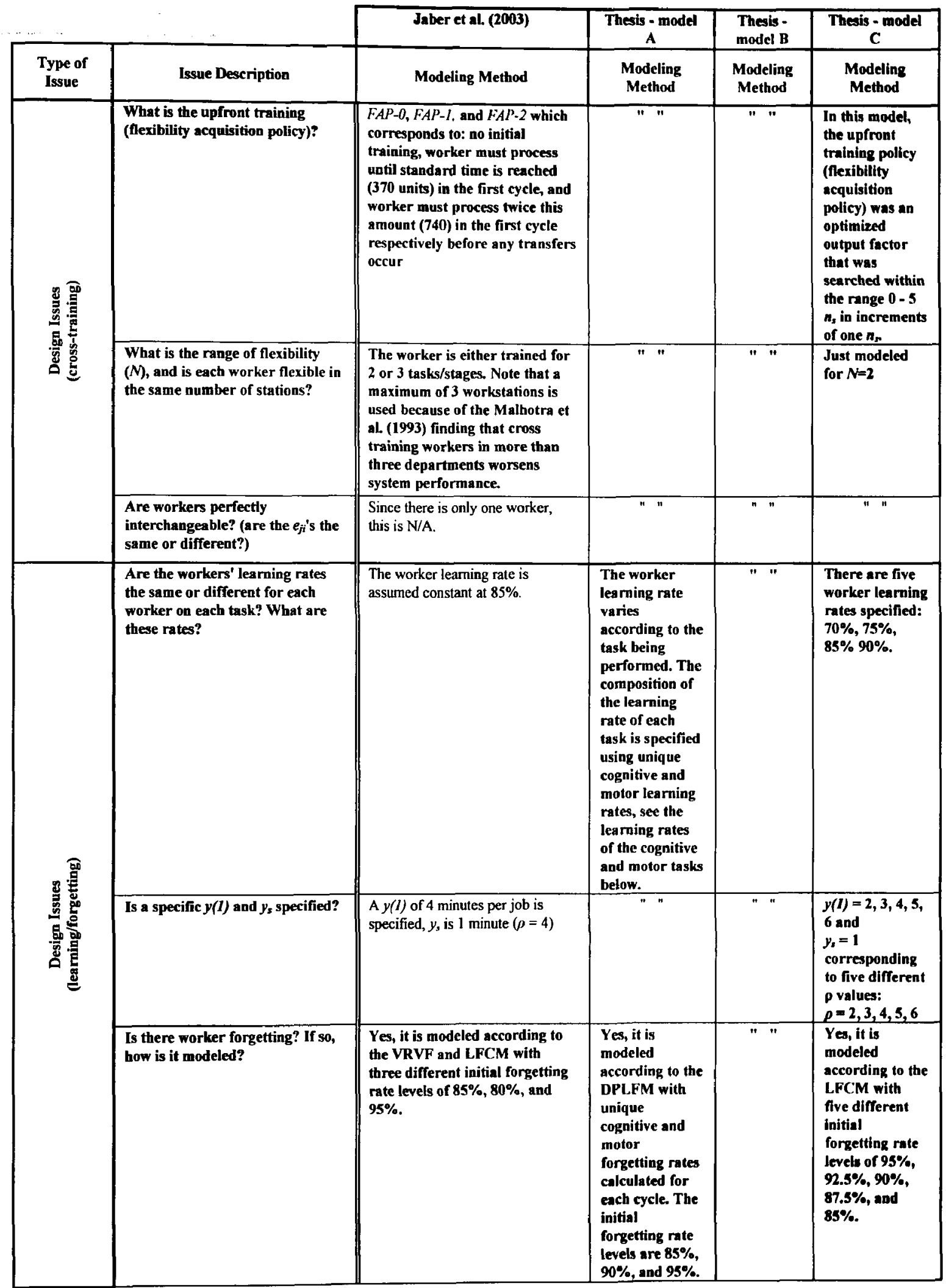




\begin{tabular}{|c|c|c|c|c|c|}
\hline & & Jaber et al. (2003) & $\begin{array}{c}\text { Thesis - model } \\
\text { A } \\
\end{array}$ & $\begin{array}{l}\text { Thesis - } \\
\text { model B }\end{array}$ & $\begin{array}{l}\text { Thesis } \\
\text { model }\end{array}$ \\
\hline Type of Insue & Issue Description & Modeling Method & $\begin{array}{c}\text { Modeling } \\
\text { Method }\end{array}$ & $\begin{array}{c}\text { Modeling } \\
\text { Method }\end{array}$ & $\begin{array}{r}\text { Modeli } \\
\text { Metho }\end{array}$ \\
\hline \multirow{5}{*}{ 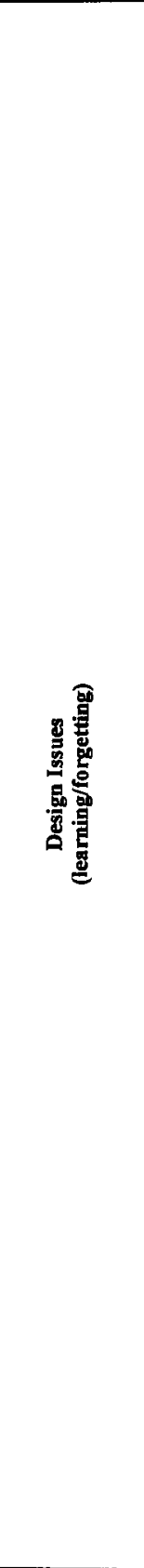 } & $\begin{array}{l}\text { What is the time to total } \\
\text { forgetting? }\end{array}$ & $\begin{array}{l}\text { The time to total forgetting }(D) \text { is a } \\
\text { function of the forgetting rate, } \\
\text { learning slope, number of units } \\
\text { produced and the time to produce } \\
\text { these units. }\end{array}$ & $\begin{array}{l}\text { The time to total } \\
\text { forgetting (D) is } \\
\text { a function of the } \\
\text { forgetting rate, } \\
\text { learning slope, } \\
\text { number of units } \\
\text { produced and the } \\
\text { time to produce } \\
\text { these units. } \\
\text { However, in the } \\
\text { DPLFM, there is } \\
\text { a unique time to } \\
\text { forgetting } \\
\text { parameter for } \\
\text { both cognitive } \\
\text { and motor } \\
\text { elements. }\end{array}$ & " " & $\begin{array}{l}\text { Same as } \\
\text { the LFC } \\
\text { model of } \\
\text { Jaber et } \\
(2003) \text {. }\end{array}$ \\
\hline & $\begin{array}{l}\text { What is the interruption } \\
\text { interval length }\left(d_{i}\right) \text { ? } \\
\text { Is this constant for every cycle } \\
\text { i? }\end{array}$ & $\begin{array}{l}\text { No, the interruption is the function } \\
\text { of the time since this station's task } \\
\text { was last processed. As a result, it } \\
\text { is variable across cycles since the } \\
\text { processing times change with time. }\end{array}$ & " " & " " & 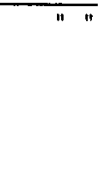 \\
\hline & $\begin{array}{l}\text { What is the initial processing } \\
\text { time cognitive to motor ratio. } \\
(R)\end{array}$ & $\begin{array}{l}\text { Not applicable in the VRVF and } \\
\text { LFCM models. }\end{array}$ & $\begin{array}{l}\text { A ratio of } 1,1 / 3 \\
\text { and } 3 \text { is } \\
\text { assumed. These } \\
\text { ratios } \\
\text { corresponds to } \\
\text { those } \\
\text { empirically } \\
\text { observed by } \\
\text { Dar El et } \\
\text { al.(1995) }\end{array}$ & " " & $\begin{array}{l}\text { Not } \\
\text { applicab } \\
\text { since the } \\
\text { LFCM u } \\
\text { used. }\end{array}$ \\
\hline & $\begin{array}{l}\text { Do workers begin forgetting as } \\
\text { soon as an interruption occurs } \\
\text { or does performance } \\
\text { temporarily remain constant } \\
\text { (or increase) shortly after an } \\
\text { interruption? Is this } \\
\text { phenomenon related to the } \\
\text { level of job complexity (as } \\
\text { defined by McKreery and } \\
\text { Krajewski) and/or cognitive } \\
\text { motor contents of the job } \\
\text { and/or the present model's } \\
\text { definition of complexity? }\end{array}$ & $\begin{array}{l}\text { Workers begin forgetting as soon } \\
\text { as an interruption occurs. }\end{array}$ & " " & " " & $1 "$ \\
\hline & $\begin{array}{l}\text { If performance improves after } \\
\text { an interruption(experimentally } \\
\text { found by Arzi and Shtub, } \\
1997 \text {, is this effect enhanced } \\
\text { by the inclusion of workshop- } \\
\text { type training during the } \\
\text { breaks of mental(cognitive) } \\
\text { tasks(suggested by Arzi and } \\
\text { Shtub, 1997) }\end{array}$ & $\begin{array}{l}\text { Workers begin forgetting as soon } \\
\text { as an interruption occurs. }\end{array}$ & $" 1$ & " n & $7 "$ \\
\hline
\end{tabular}




\begin{tabular}{|c|c|c|c|c|c|}
\hline & & Jaber et al. (2003) & $\begin{array}{c}\text { Thesis - model } \\
\text { A }\end{array}$ & $\begin{array}{c}\text { Thesis - model } \\
\text { B }\end{array}$ & Thesis - model \\
\hline Type of Issue & Issue Description & Modeling Method & $\begin{array}{l}\text { Modeling } \\
\text { Method }\end{array}$ & $\begin{array}{l}\text { Modeling } \\
\text { Method }\end{array}$ & $\begin{array}{c}\text { Mlodeling } \\
\text { Method }\end{array}$ \\
\hline \multirow{7}{*}{ 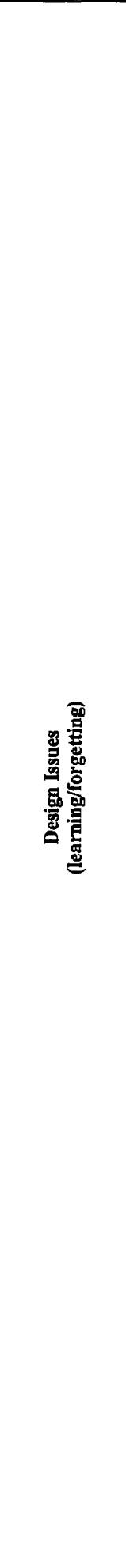 } & $\begin{array}{l}\text { Does the model distinguish } \\
\text { between cognitive and motor } \\
\text { aspect of a job? And if so, is } \\
\text { the relative arrangement or } \\
\text { sequence of the jobs (i.e: } \\
\text { cognitive-motor-agnitive, for } \\
\text { example) specified? }\end{array}$ & $\begin{array}{l}\text { No, cognitive and/or motor } \\
\text { elements of the tasks are not } \\
\text { mentioned. }\end{array}$ & 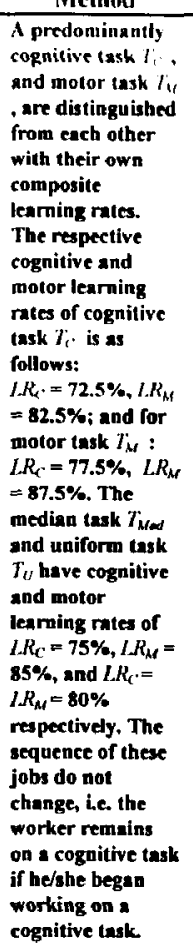 & 411 & $\begin{array}{l}\text { No, connitice } \\
\text { and/or motor } \\
\text { elements of the } \\
\text { tasks are not } \\
\text { mentioned since } \\
\text { the LFCM was } \\
\text { used. }\end{array}$ \\
\hline & $\begin{array}{l}\text { Are the workers modeled as } \\
\text { heterogeneous or homogeneous } \\
\text { with respect } \\
\text { learning/forgetting } \\
\text { distributions (see Shafer et al., } \\
2001 \text { )? }\end{array}$ & Only one worker, therefore N/A. & $" 1$ & " " & $n$ \\
\hline & $\begin{array}{l}\text { Is the degree of learning } \\
\text { and/or forgetting a function of } \\
\text { the complexity of the task } \\
\text { and/or the individual worker- } \\
\text { perceived complexity? }\end{array}$ & $\begin{array}{l}\text { No relationship between task } \\
\text { complexity and learning/forgetting } \\
\text { suggested. }\end{array}$ & $7 "$ & $7 "$ & " " \\
\hline & $\begin{array}{l}\text { Does this model allow for } \\
\text { negative forgetting? (see } \\
\text { Nembhard and Uzumeri } 2000 \text {, } \\
25(4), 315-326 \text {. }\end{array}$ & No. & $" 1$ & " " & " \\
\hline & $\begin{array}{l}\text { Does the size of the lots of the } \\
\text { products interact with the } \\
\text { 'product' vs. 'product' } \\
\text { learning/forgetting variables? } \\
\text { (i.e.: try a lot size of } 1 \text { as an } \\
\text { extreme case of make to order; } \\
\text { what about a variable lot size } \\
\text { per product?) }\end{array}$ & $\begin{array}{l}\text { No, product or process distinction } \\
\text { is not made. }\end{array}$ & " & " " & $" n$ \\
\hline & $\begin{array}{l}\text { Does the paper } \\
\text { distinguish(qualitatively and/or } \\
\text { mathematically) between } \\
\text { 'product' vs. 'process' learning } \\
\text { as in McKreery and } \\
\text { Krajewski? }\end{array}$ & $\begin{array}{l}\text { No, product or process distinction } \\
\text { is not made. }\end{array}$ & " " & 4 & $7 "$ \\
\hline & $\begin{array}{l}\text { What fraction of worker } \\
\text { learning is 'process' and what } \\
\text { fraction is 'product' learning? }\end{array}$ & $\begin{array}{l}\text { Product or process distinction is } \\
\text { not made. }\end{array}$ & " " & " " & \\
\hline
\end{tabular}




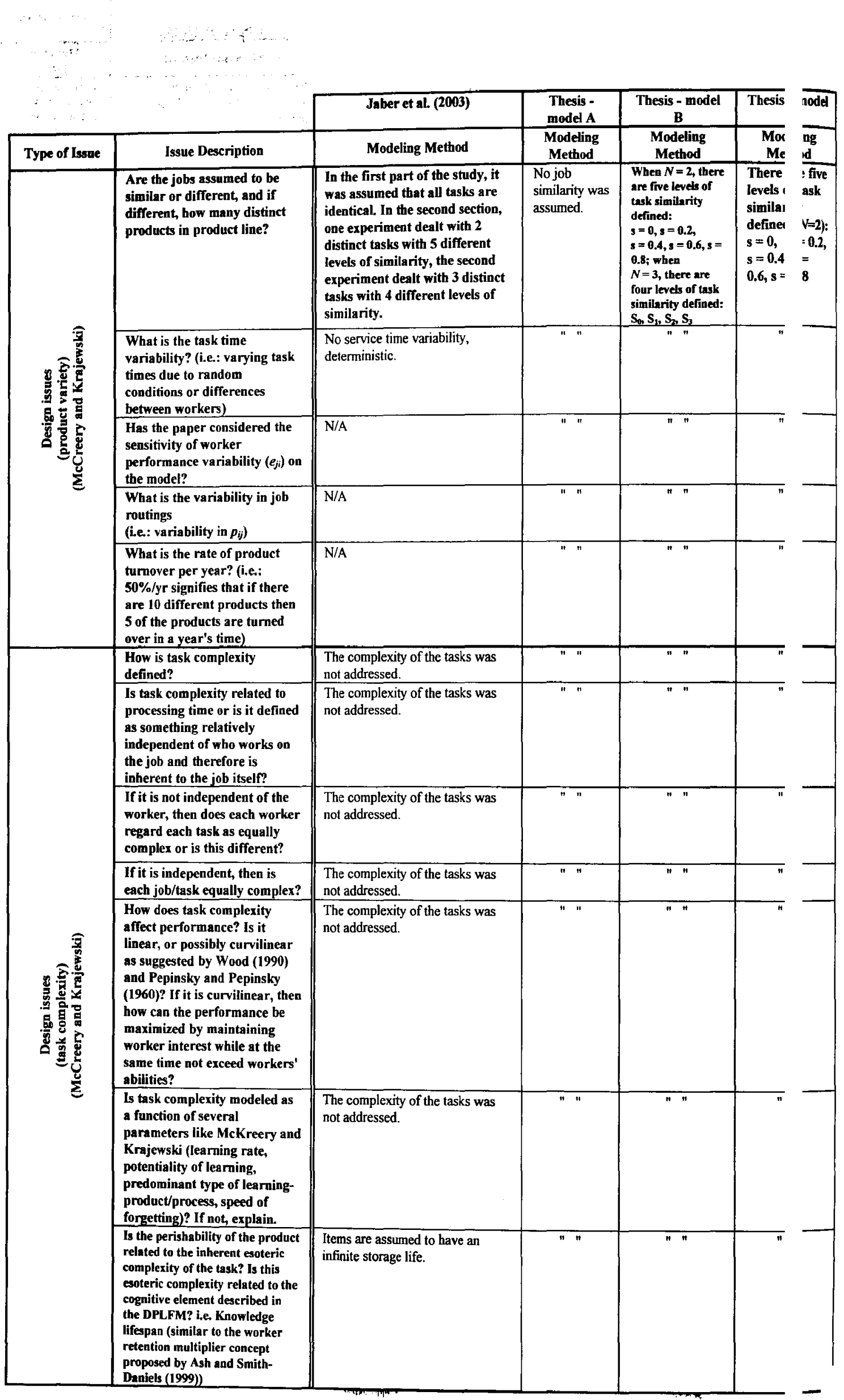




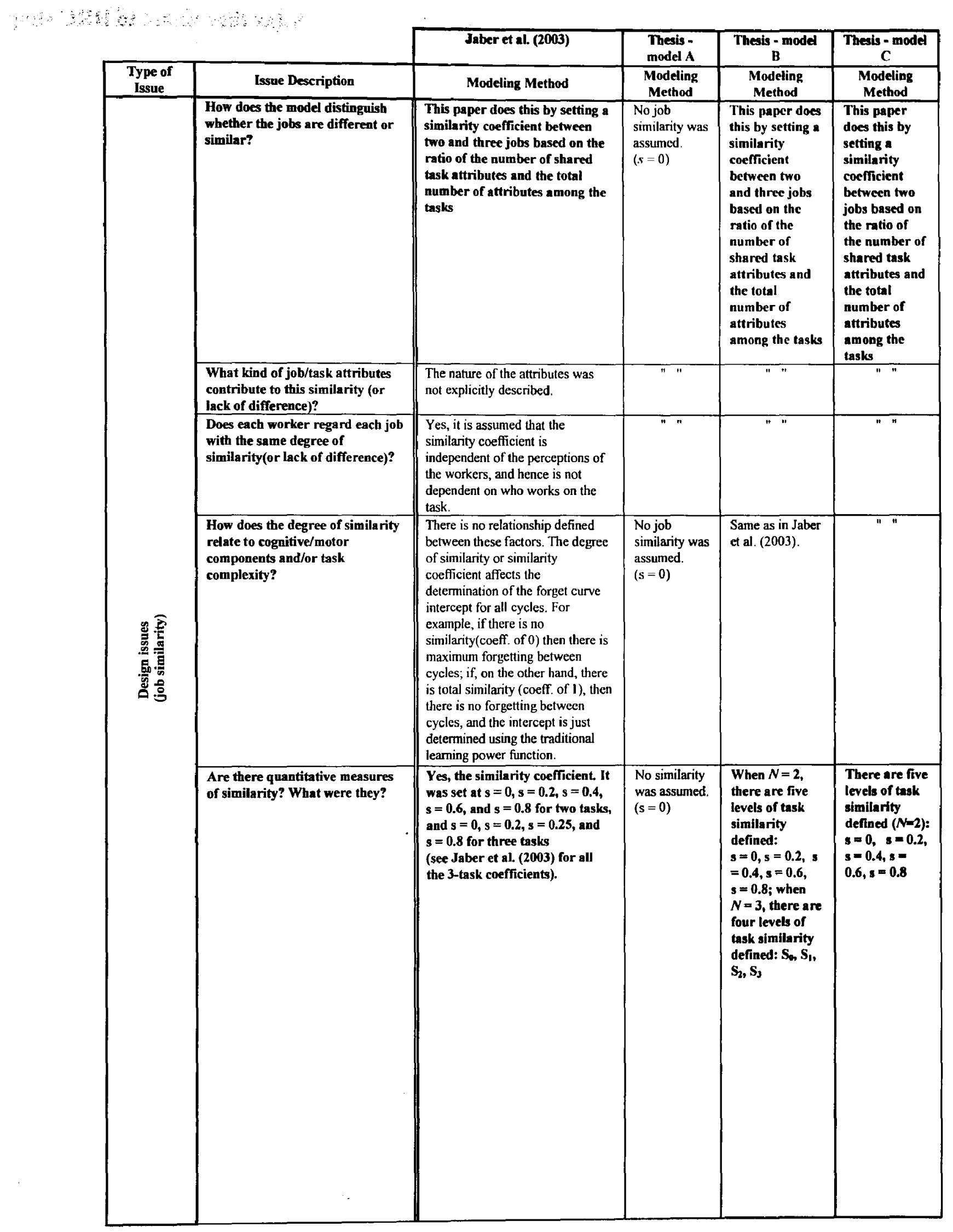




\subsubsection{Model results/conclusions (as they relate to DRC shop}

\section{performance measures: categorized by experimental factors}

\section{examined)}

\begin{tabular}{|c|c|c|c|c|}
\hline & Jaber et al. (2003) & $\begin{array}{l}\text { Thesis - } \\
\text { model A }\end{array}$ & $\begin{array}{l}\text { Thesis - } \\
\text { model B }\end{array}$ & $\begin{array}{l}\text { Thesis } \\
\text { model }(\end{array}$ \\
\hline Type of Issue & Modeling Method & $\begin{array}{l}\text { Modeling } \\
\text { Method }\end{array}$ & $\begin{array}{l}\text { Modeling } \\
\text { Method }\end{array}$ & $\begin{array}{r}\text { Modelin } \\
\text { Method }\end{array}$ \\
\hline $\begin{array}{l}\text { Worker flexibility } \\
\text { results/conclusions } \\
\text { (level of cross training) }\end{array}$ & $\begin{array}{l}\text { It was concluded that training workers for } \\
\text { more than } 2 \text { different tasks ( } 3 \text { tasks) is not } \\
\text { necessary because the forgetting losses for } \\
\text { learning } 3 \text { tasks are greater than learning } 2 \\
\text { tasks. }\end{array}$ & " " & $\pi "$ & $\overline{~ " ~ " ~}$ \\
\hline $\begin{array}{l}\text { Centralization of control } \\
\text { results/conclusions } \\
\text { ('when' rule) }\end{array}$ & $\begin{array}{l}\text { Out of the possible levels of } 10,25 \text { and } 250 \text {, } \\
\text { Overall, a worker transfer policy of } 250 \text { was } \\
\text { optimal. These results differed from those of } \\
\text { Hottenstein and Bowman (1998) because } \\
\text { they concluded that a centralized policy was } \\
\text { superior to a decentralized policy; albeit, } \\
\text { Hottenstein and Bowman (1998) concluded } \\
\text { that is was superior by only a marginal } \\
\text { amount. }\end{array}$ & 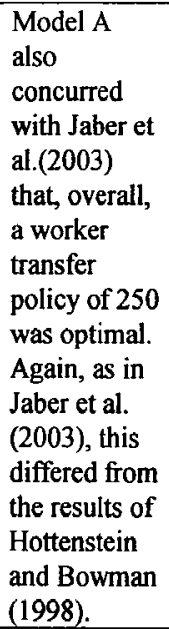 & $7 "$ & $" 1 "$ \\
\hline $\begin{array}{c}\text { Worker assignment rule } \\
\text { resnits/conclusions } \\
\text { ('where' rule) }\end{array}$ & This experimental factor was not examined. & $" 1$ & " " & " " \\
\hline $\begin{array}{l}\text { Queue discipline rule } \\
\text { results/conclusions }\end{array}$ & $\begin{array}{l}\text { There are no arrivals until a unit is complete, } \\
\text { therefore it is a simple static, first-come-first- } \\
\text { serve model. As a result, the comparison } \\
\text { between different queue discipline rules was } \\
\text { not made. }\end{array}$ & " " & " " & " \\
\hline $\begin{array}{c}\text { Cost of transferring } \\
\text { workers } \\
\text { results/conclusions }\end{array}$ & $\begin{array}{l}\text { There are no conclusions made since the } \\
\text { costs are assumed to be zero. }\end{array}$ & " " & " " & \\
\hline
\end{tabular}


7.2 Model A results

7.2.1 DPLFM results (APT)

$$
N=2, F R=95 \%
$$

\begin{tabular}{|c|c|c|c|c|c|}
\hline$C$ & Task Type & $R$ & $F A P-0$ & $F A P-1$ & $F A P-2$ \\
\hline \multirow{12}{*}{10} & \multirow{3}{*}{$T_{C}$} & $1 / 3$ & 1.0231 & 1.0008 & 1.0137 \\
\hline & & 1 & 1.0150 & 1.0010 & 1.0100 \\
\hline & & 3 & 1.0110 & 1.0012 & 1.0079 \\
\hline & \multirow{3}{*}{$T_{M}$} & $1 / 3$ & 1.3609 & 1.0009 & 1.0516 \\
\hline & & 1 & 1.0495 & 1.0006 & 1.0254 \\
\hline & & 3 & 1.0214 & 1.0008 & 1.0141 \\
\hline & \multirow{3}{*}{$T_{\text {Med }}$} & $1 / 3$ & 1.0498 & 1.0006 & 1.0231 \\
\hline & & 1 & 1.0222 & 1.0008 & 1.0145 \\
\hline & & 3 & 1.0144 & 1.0010 & 1.0099 \\
\hline & \multirow{3}{*}{$T_{U}$} & $1 / 3$ & 1.0205 & 1.0010 & 1.0125 \\
\hline & & 1 & 1.0219 & 1.0011 & 1.0126 \\
\hline & & 3 & 1.0205 & 1.0010 & 1.0125 \\
\hline \multirow{12}{*}{25} & \multirow{3}{*}{$T_{C}$} & $1 / 3$ & 1.0180 & 1.0014 & 1.0142 \\
\hline & & 1 & 1.0123 & 1.0015 & 1.0104 \\
\hline & & 3 & 1.0091 & 1.0017 & 1.0082 \\
\hline & \multirow{3}{*}{$T_{M}$} & $1 / 3$ & 1.0867 & 1.0011 & 1.0521 \\
\hline & & 1 & 1.0329 & 1.0010 & 1.0259 \\
\hline & & 3 & 1.0177 & 1.0013 & 1.0146 \\
\hline & \multirow{3}{*}{$T_{\text {Med }}$} & $1 / 3$ & 1.0311 & 1.0010 & 1.0236 \\
\hline & & 1 & 1.0182 & 1.0013 & 1.0150 \\
\hline & & 3 & 1.0120 & 1.0016 & 1.0103 \\
\hline & \multirow{3}{*}{$T_{U}$} & $1 / 3$ & 1.0166 & 1.0017 & 1.0130 \\
\hline & & 1 & 1.0171 & 1.0018 & 1.0131 \\
\hline & & 3 & 1.0166 & 1.0017 & 1.0130 \\
\hline \multirow{12}{*}{250} & \multirow{3}{*}{$T_{C}$} & $1 / 3$ & 1.0141 & 1.0016 & 1.0158 \\
\hline & & 1 & 1.0099 & 1.0013 & 1.0115 \\
\hline & & 3 & 1.0075 & 1.0011 & 1.0089 \\
\hline & \multirow{3}{*}{$T_{M}$} & $1 / 3$ & 1.0552 & 1.0020 & 1.0612 \\
\hline & & 1 & 1.0260 & 1.0018 & 1.0293 \\
\hline & & 3 & 1.0144 & 1.0015 & 1.0161 \\
\hline & \multirow{3}{*}{$T_{\text {Med }}$} & $1 / 3$ & 1.0240 & 1.0019 & 1.0272 \\
\hline & & 1 & 1.0148 & 1.0015 & 1.0165 \\
\hline & & 3 & 1.0098 & 1.0012 & 1.0114 \\
\hline & \multirow{3}{*}{$T_{U}$} & $1 / 3$ & 1.0126 & 1.0017 & 1.0145 \\
\hline & & 1 & 1.0127 & 1.0018 & 1.0145 \\
\hline & & 3 & 1.0126 & 1.0017 & 1.0145 \\
\hline
\end{tabular}




$$
N=2, F R=90 \%
$$

\begin{tabular}{|c|c|c|c|c|c|}
\hline$C$ & Task Type & $R$ & $F A P-0$ & $F A P-1$ & $F A P-2$ \\
\hline \multirow{12}{*}{10} & \multirow{3}{*}{$T_{C}$} & $1 / 3$ & 1.3784 & 1.0048 & 1.0164 \\
\hline & & 1 & 1.0814 & 1.0055 & 1.0128 \\
\hline & & 3 & 1.0302 & 1.0059 & 1.0106 \\
\hline & \multirow{3}{*}{$T_{M}$} & $1 / 3$ & 2.1088 & 1.0070 & 1.0566 \\
\hline & & 1 & 1.6664 & 1.0034 & 1.0276 \\
\hline & & 3 & 1.2797 & 1.0038 & 1.0161 \\
\hline & \multirow{3}{*}{$T_{\text {Med }}$} & $1 / 3$ & 1.7298 & 1.0041 & 1.0258 \\
\hline & & 1 & 1.3600 & 1.0040 & 1.0167 \\
\hline & & 3 & 1.0441 & 1.0049 & 1.0123 \\
\hline & \multirow{3}{*}{$T_{U}$} & $1 / 3$ & 1.2673 & 1.0046 & 1.0149 \\
\hline & & 1 & 1.3750 & 1.0053 & 1.0153 \\
\hline & & 3 & 1.2673 & 1.0046 & 1.0149 \\
\hline \multirow{12}{*}{25} & \multirow{3}{*}{$T_{C}$} & $1 / 3$ & 1.0453 & 1.0083 & 1.0186 \\
\hline & & 1 & 1.0277 & 1.0088 & 1.0147 \\
\hline & & 3 & 1.0175 & 1.0084 & 1.0121 \\
\hline & \multirow{3}{*}{$T_{M}$} & $1 / 3$ & 1.6000 & 1.0075 & 1.0573 \\
\hline & & 1 & 1.2155 & 1.0052 & 1.0289 \\
\hline & & 3 & 1.0376 & 1.0065 & 1.0179 \\
\hline & \multirow{3}{*}{$T_{\text {Med }}$} & $1 / 3$ & 1.2672 & 1.0063 & 1.0272 \\
\hline & & 1 & 1.0425 & 1.0069 & 1.0185 \\
\hline & & 3 & 1.0244 & 1.0077 & 1.0140 \\
\hline & \multirow{3}{*}{$T_{U}$} & $1 / 3$ & 1.0365 & 1.0077 & 1.0168 \\
\hline & & 1 & 1.0430 & 1.0090 & 1.0175 \\
\hline & & 3 & 1.0365 & 1.0077 & 1.0168 \\
\hline \multirow{12}{*}{250} & \multirow{3}{*}{$T_{C}$} & $1 / 3$ & 1.0182 & 1.0101 & 1.0211 \\
\hline & & 1 & 1.0122 & 1.0083 & 1.0156 \\
\hline & & 3 & 1.0087 & 1.0068 & 1.0120 \\
\hline & \multirow{3}{*}{$T_{M}$} & $1 / 3$ & 1.0760 & 1.0110 & 1.0672 \\
\hline & & 1 & 1.0333 & 1.0098 & 1.0346 \\
\hline & & 3 & 1.0178 & 1.0081 & 1.0203 \\
\hline & \multirow{3}{*}{$T_{\text {Med }}$} & $1 / 3$ & 1.0318 & 1.0113 & 1.0332 \\
\hline & & 1 & 1.0186 & 1.0088 & 1.0211 \\
\hline & & 3 & 1.0117 & 1.0072 & 1.0149 \\
\hline & \multirow{3}{*}{$T_{U}$} & $1 / 3$ & 1.0156 & 1.0083 & 1.0186 \\
\hline & & 1 & 1.0160 & 1.0097 & 1.0192 \\
\hline & & 3 & 1.0156 & 1.0083 & 1.0186 \\
\hline
\end{tabular}




$$
N=2, F R=85 \%
$$

\begin{tabular}{|c|c|c|c|c|c|}
\hline$C$ & Task Type & $R$ & $F A P-0$ & $F A P-1$ & $F A P-2$ \\
\hline \multirow{12}{*}{10} & \multirow{3}{*}{$T_{C}$} & $1 / 3$ & 1.7251 & 1.0128 & 1.0212 \\
\hline & & 1 & 1.4919 & 1.0167 & 1.0188 \\
\hline & & 3 & 1.0835 & 1.0159 & 1.0159 \\
\hline & \multirow{3}{*}{$T_{M}$} & $1 / 3$ & 2.3914 & 1.0869 & 1.0805 \\
\hline & & 1 & 2.0386 & 1.0130 & 1.0335 \\
\hline & & 3 & 1.6370 & 1.0103 & 1.0202 \\
\hline & \multirow{3}{*}{$T_{\text {Med }}$} & $1 / 3$ & 2.0511 & 1.0161 & 1.0326 \\
\hline & & 1 & 1.7541 & 1.0123 & 1.0216 \\
\hline & & 3 & 1.3595 & 1.0132 & 1.0170 \\
\hline & \multirow{3}{*}{$T_{U}$} & $1 / 3$ & 1.6324 & 1.0121 & 1.0194 \\
\hline & & 1 & 1.7746 & 1.0155 & 1.0210 \\
\hline & & 3 & 1.6324 & 1.0121 & 1.0194 \\
\hline \multirow{12}{*}{25} & \multirow{3}{*}{$T_{C}$} & $1 / 3$ & 1.2281 & 1.0218 & 1.0262 \\
\hline & & 1 & 1.0735 & 1.0269 & 1.0240 \\
\hline & & 3 & 1.0365 & 1.0230 & 1.0196 \\
\hline & \multirow{3}{*}{$T_{M}$} & $1 / 3$ & 1.9010 & 1.0785 & 1.0794 \\
\hline & & 1 & 1.5409 & 1.0170 & 1.0362 \\
\hline & & 3 & 1.1716 & 1.0177 & 1.0245 \\
\hline & \multirow{3}{*}{$T_{\text {Med }}$} & $1 / 3$ & 1.5471 & 1.0206 & 1.0359 \\
\hline & & 1 & 1.2574 & 1.0210 & 1.0265 \\
\hline & & 3 & 1.0548 & 1.0211 & 1.0213 \\
\hline & \multirow{3}{*}{$T_{U}$} & $1 / 3$ & 1.1227 & 1.0205 & 1.0241 \\
\hline & & 1 & 1.2250 & 1.0261 & 1.0265 \\
\hline & & $\mathbf{3}$ & 1.1227 & 1.0205 & 1.0241 \\
\hline \multirow{12}{*}{250} & \multirow{3}{*}{$T_{C}$} & $1 / 3$ & 1.0250 & 1.0264 & 1.0302 \\
\hline & & 1 & 1.0169 & 1.0271 & 1.0253 \\
\hline & & 3 & 1.0111 & 1.0213 & 1.0192 \\
\hline & \multirow{3}{*}{$T_{M}$} & $1 / 3$ & 1.1506 & 1.0368 & 1.0826 \\
\hline & & 1 & 1.0498 & 1.0285 & 1.0456 \\
\hline & & 3 & 1.0242 & 1.0223 & 1.0285 \\
\hline & \multirow{3}{*}{$T_{\text {Med }}$} & $1 / 3$ & 1.0486 & 1.0322 & 1.0456 \\
\hline & & 1 & 1.0266 & 1.0267 & 1.0311 \\
\hline & & 3 & 1.0154 & 1.0209 & 1.0222 \\
\hline & \multirow{3}{*}{$T_{U}$} & $1 / 3$ & 1.0209 & 1.0224 & 1.0264 \\
\hline & & 1 & 1.0225 & 1.0282 & 1.0291 \\
\hline & & 3 & 1.0209 & 1.0224 & 1.0264 \\
\hline
\end{tabular}


$N=3, F R=95 \%$

\begin{tabular}{|c|c|c|c|c|c|}
\hline$C$ & Task Type & $R$ & $F A P-0$ & $F A P-1$ & $F A P-2$ \\
\hline \multirow{12}{*}{10} & \multirow{3}{*}{$T_{C}$} & $1 / 3$ & 1.1263 & 1.0030 & 1.0152 \\
\hline & & 1 & 1.0284 & 1.0036 & 1.0118 \\
\hline & & 3 & 1.0196 & 1.0041 & 1.0097 \\
\hline & \multirow{3}{*}{$T_{M}$} & $1 / 3$ & 1.7488 & 1.0032 & 1.0534 \\
\hline & & 1 & 1.3887 & 1.0022 & 1.0266 \\
\hline & & 3 & 1.0469 & 1.0028 & 1.0155 \\
\hline & \multirow{3}{*}{$T_{\text {Med }}$} & $1 / 3$ & 1.4156 & 1.0023 & 1.0244 \\
\hline & & 1 & 1.0811 & 1.0028 & 1.0159 \\
\hline & & 3 & 1.0265 & 1.0036 & 1.0116 \\
\hline & \multirow{3}{*}{$T_{U}$} & $1 / 3$ & 1.0439 & 1.0035 & 1.0143 \\
\hline & & 1 & 1.0850 & 1.0038 & 1.0144 \\
\hline & & 3 & 1.0439 & 1.0035 & 1.0143 \\
\hline \multirow{12}{*}{25} & \multirow{3}{*}{$T_{C}$} & $1 / 3$ & 1.0281 & 1.0049 & 1.0165 \\
\hline & & 1 & 1.0186 & 1.0051 & 1.0127 \\
\hline & & 3 & 1.0131 & 1.0051 & 1.0104 \\
\hline & \multirow{3}{*}{$T_{M}$} & $1 / 3$ & 1.3159 & 1.0036 & 1.0541 \\
\hline & & 1 & 1.0548 & 1.0034 & 1.0276 \\
\hline & & 3 & 1.0268 & 1.0045 & 1.0168 \\
\hline & \multirow{3}{*}{$T_{\text {Med }}$} & $1 / 3$ & 1.0519 & 1.0036 & 1.0254 \\
\hline & & 1 & 1.0276 & 1.0044 & 1.0171 \\
\hline & & 3 & 1.0179 & 1.0050 & 1.0126 \\
\hline & \multirow{3}{*}{$T_{U}$} & $1 / 3$ & 1.0259 & 1.0054 & 1.0156 \\
\hline & & 1 & 1.0274 & 1.0058 & 1.0158 \\
\hline & & 3 & 1.0259 & 1.0054 & 1.0156 \\
\hline \multirow{12}{*}{250} & \multirow{3}{*}{$T_{C}$} & $1 / 3$ & 1.0156 & 1.0043 & 1.0176 \\
\hline & & 1 & 1.0108 & 1.0033 & 1.0130 \\
\hline & & 3 & 1.0080 & 1.0028 & 1.0100 \\
\hline & \multirow{3}{*}{$T_{M}$} & $1 / 3$ & 1.0629 & 1.0057 & 1.0637 \\
\hline & & 1 & 1.0291 & 1.0051 & 1.0316 \\
\hline & & 3 & 1.0159 & 1.0041 & 1.0179 \\
\hline & \multirow{3}{*}{$T_{\text {Med }}$} & $1 / 3$ & 1.0268 & 1.0051 & 1.0295 \\
\hline & & 1 & 1.0164 & 1.0041 & 1.0183 \\
\hline & & 3 & 1.0106 & 1.0032 & 1.0127 \\
\hline & \multirow{3}{*}{$T_{U}$} & $1 / 3$ & 1.0140 & 1.0042 & 1.0162 \\
\hline & & 1 & 1.0141 & 1.0045 & 1.0164 \\
\hline & & 3 & 1.0140 & 1.0042 & 1.0162 \\
\hline
\end{tabular}




$$
N=3, F R=90 \%
$$

\begin{tabular}{|c|c|c|c|c|c|}
\hline$C$ & Task Type & $R$ & $F A P-0$ & $F A P-1$ & $F A P-2$ \\
\hline \multirow{12}{*}{10} & \multirow{3}{*}{$T_{C}$} & $1 / 3$ & 1.7420 & 1.0197 & 1.0254 \\
\hline & & 1 & 1.4647 & 1.0198 & 1.0214 \\
\hline & & 3 & 1.0833 & 1.0198 & 1.0189 \\
\hline & \multirow{3}{*}{$T_{M}$} & $1 / 3$ & 2.3971 & 1.1351 & 1.1079 \\
\hline & & 1 & 2.0008 & 1.0543 & 1.0370 \\
\hline & & 3 & 1.6476 & 1.0147 & 1.0232 \\
\hline & \multirow{3}{*}{$T_{\text {Med }}$} & $1 / 3$ & 2.0616 & 1.0919 & 1.0371 \\
\hline & & 1 & 1.7204 & 1.0159 & 1.0241 \\
\hline & & 3 & 1.3773 & 1.0174 & 1.0201 \\
\hline & \multirow{3}{*}{$T_{U}$} & $1 / 3$ & 1.6480 & 1.0174 & 1.0231 \\
\hline & & 1 & 1.7472 & 1.0206 & 1.0246 \\
\hline & & 3 & 1.6480 & 1.0174 & 1.0231 \\
\hline \multirow{12}{*}{25} & \multirow{3}{*}{$T_{C}$} & $1 / 3$ & 1.2385 & 1.0287 & 1.0313 \\
\hline & & 1 & 1.0653 & 1.0276 & 1.0260 \\
\hline & & 3 & 1.0369 & 1.0242 & 1.0215 \\
\hline & \multirow{3}{*}{$T_{M}$} & $1 / 3$ & 1.8715 & 1.1131 & 1.0990 \\
\hline & & 1 & 1.5121 & 1.0235 & 1.0394 \\
\hline & & 3 & 1.1711 & 1.0223 & 1.0281 \\
\hline & \multirow{3}{*}{$T_{\text {Med }}$} & $1 / 3$ & 1.5616 & 1.0346 & 1.0395 \\
\hline & & 1 & 1.2253 & 1.0238 & 1.0292 \\
\hline & & 3 & 1.0552 & 1.0241 & 1.0241 \\
\hline & \multirow{3}{*}{$T_{U}$} & $1 / 3$ & 1.1251 & 1.0258 & 1.0282 \\
\hline & & 1 & 1.1932 & 1.0301 & 1.0303 \\
\hline & & 3 & 1.1251 & 1.0258 & 1.0282 \\
\hline \multirow{12}{*}{250} & \multirow{3}{*}{$T_{C}$} & $1 / 3$ & 1.0253 & 1.0231 & 1.0298 \\
\hline & & 1 & 1.0160 & 1.0184 & 1.0220 \\
\hline & & 3 & 1.0109 & 1.0147 & 1.0168 \\
\hline & \multirow{3}{*}{$T_{M}$} & $1 / 3$ & 1.1191 & 1.0313 & 1.0798 \\
\hline & & 1 & 1.0474 & 1.0253 & 1.0450 \\
\hline & & 3 & 1.0240 & 1.0190 & 1.0277 \\
\hline & \multirow{3}{*}{$T_{\text {Med }}$} & $1 / 3$ & 1.0461 & 1.0283 & 1.0443 \\
\hline & & 1 & 1.0253 & 1.0207 & 1.0290 \\
\hline & & 3 & 1.0152 & 1.0160 & 1.0206 \\
\hline & \multirow{3}{*}{$T_{U}$} & $1 / 3$ & 1.0209 & 1.0189 & 1.0256 \\
\hline & & 1 & 1.0219 & 1.0217 & 1.0271 \\
\hline & & 3 & 1.0209 & 1.0189 & 1.0256 \\
\hline
\end{tabular}




$$
N=3, F R=85 \%
$$

\begin{tabular}{|c|c|c|c|c|c|}
\hline$C$ & Task Type & $R$ & $F A P-0$ & $F A P-1$ & $F A P-2$ \\
\hline \multirow{12}{*}{10} & \multirow{3}{*}{$T_{C}$} & $1 / 3$ & 2.0664 & 1.2708 & 1.1274 \\
\hline & & 1 & 1.8559 & 1.1903 & 1.0438 \\
\hline & & 3 & 1.4711 & 1.0541 & 1.0366 \\
\hline & \multirow{3}{*}{$T_{M}$} & $1 / 3$ & 2.6494 & 1.4259 & 1.3012 \\
\hline & & 1 & 2.3408 & 1.3416 & 1.2275 \\
\hline & & 3 & 1.9818 & 1.2181 & 1.0805 \\
\hline & \multirow{3}{*}{$T_{\text {Med }}$} & $1 / 3$ & 2.3549 & 1.3814 & 1.2599 \\
\hline & & 1 & 2.0906 & 1.2697 & 1.1301 \\
\hline & & 3 & 1.7287 & 1.1005 & 1.0370 \\
\hline & \multirow{3}{*}{$T_{U}$} & $1 / 3$ & 1.9866 & 1.2271 & 1.0619 \\
\hline & & 1 & 2.1198 & 1.2937 & 1.1291 \\
\hline & & 3 & 1.9866 & 1.2271 & 1.0619 \\
\hline \multirow{12}{*}{25} & \multirow{3}{*}{$T_{C}$} & $1 / 3$ & 1.5078 & 1.1533 & 1.0579 \\
\hline & & 1 & 1.2545 & 1.0828 & 1.0548 \\
\hline & & 3 & 1.0870 & 1.0638 & 1.0426 \\
\hline & \multirow{3}{*}{$T_{M}$} & $1 / 3$ & 2.1302 & 1.3605 & 1.2634 \\
\hline & & 1 & 1.8015 & 1.2623 & 1.1718 \\
\hline & & 3 & 1.4555 & 1.1062 & 1.0510 \\
\hline & \multirow{3}{*}{$T_{\text {Med }}$} & $1 / 3$ & 1.8090 & 1.2912 & 1.1936 \\
\hline & & 1 & 1.5335 & 1.1612 & 1.0576 \\
\hline & & 3 & 1.1561 & 1.0647 & 1.0463 \\
\hline & \multirow{3}{*}{$T_{U}$} & $1 / 3$ & 1.4220 & 1.0879 & 1.0523 \\
\hline & & 1 & 1.5065 & 1.1561 & 1.0608 \\
\hline & & 3 & 1.4220 & 1.0879 & 1.0523 \\
\hline \multirow{12}{*}{250} & \multirow{3}{*}{$T_{C}$} & $1 / 3$ & 1.0395 & 1.0525 & 1.0481 \\
\hline & & 1 & 1.0259 & 1.0494 & 1.0406 \\
\hline & & 3 & 1.0160 & 1.0381 & 1.0303 \\
\hline & \multirow{3}{*}{$T_{M}$} & $1 / 3$ & 1.2789 & 1.1234 & 1.1253 \\
\hline & & 1 & 1.0836 & 1.0658 & 1.0702 \\
\hline & & 3 & 1.0377 & 1.0462 & 1.0448 \\
\hline & \multirow{3}{*}{$T_{\text {Med }}$} & $1 / 3$ & 1.0818 & 1.0711 & 1.0717 \\
\hline & & 1 & 1.0422 & 1.0540 & 1.0496 \\
\hline & & 3 & 1.0230 & 1.0401 & 1.0351 \\
\hline & \multirow{3}{*}{$T_{U}$} & $1 / 3$ & 1.0323 & 1.0447 & 1.0415 \\
\hline & & 1 & 1.0355 & 1.0538 & 1.0465 \\
\hline & & 3 & 1.0323 & 1.0447 & 1.0415 \\
\hline
\end{tabular}




\subsubsection{LFCM and VRVF results (APT)}

\begin{tabular}{|c|c|c|c|c|c|c|c|c|}
\hline \multirow[b]{2}{*}{$N$} & \multirow[b]{2}{*}{$F R$} & \multirow[b]{2}{*}{$C$} & \multicolumn{3}{|c|}{$\begin{array}{l}\text { LFCM } \\
\text { (Jaber et al., 2003) }\end{array}$} & \multicolumn{3}{|c|}{$\begin{array}{l}\text { VRVF } \\
\text { (Jaber et al., 2003) }\end{array}$} \\
\hline & & & $F A P-0$ & $F A P-1$ & $F A P-2$ & $F A P-0$ & $F A P-1$ & $F A P-2$ \\
\hline \multirow{9}{*}{2} & \multirow{3}{*}{$\begin{array}{l}\text { Low } \\
(95 \%)\end{array}$} & 10 & 1.3075 & 1.0011 & 1.0390 & 1.0516 & 1.0011 & 1.0391 \\
\hline & & 25 & 1.0675 & 1.0675 & 1.0675 & 1.0675 & 1.0675 & 1.0675 \\
\hline & & 250 & 1.0431 & 1.0028 & 1.0433 & 1.0422 & 1.0024 & 1.0435 \\
\hline & \multirow{3}{*}{$\begin{array}{l}\text { Med } \\
(90 \%)\end{array}$} & 10 & 1.8990 & 1.0065 & 1.0432 & 1.0848 & 1.0071 & 1.0450 \\
\hline & & 25 & 1.4478 & 1.0075 & 1.0441 & 1.0755 & 1.0078 & 1.0458 \\
\hline & & 250 & 1.0566 & 1.0125 & 1.0494 & 1.0522 & 1.0107 & 1.0504 \\
\hline & \multirow{3}{*}{$\begin{array}{l}\text { High } \\
(85 \%)\end{array}$} & 10 & 2.1925 & 1.0486 & 1.0532 & 1.3201 & 1.1196 & 1.0962 \\
\hline & & 25 & 1.7187 & 1.0379 & 1.0541 & 1.2008 & 1.0753 & 1.0766 \\
\hline & & 250 & 1.0817 & 1.0314 & 1.0593 & 1.0696 & 1.0273 & 1.0631 \\
\hline \multirow{9}{*}{3} & \multirow{3}{*}{$\begin{array}{l}\text { Low } \\
(95 \%)\end{array}$} & 10 & 1.7138 & 1.0042 & 1.0415 & 1.0774 & 1.0044 & 1.0424 \\
\hline & & 25 & 1.2960 & 1.0051 & 1.0423 & 1.0683 & 1.0052 & 1.0433 \\
\hline & & 250 & 1.0501 & 1.0078 & 1.0468 & 1.0477 & 1.0069 & 1.0473 \\
\hline & \multirow{3}{*}{$\begin{array}{l}\text { Med } \\
(90 \%)\end{array}$} & 10 & 2.2197 & 1.1722 & 1.0958 & 1.7301 & 1.5305 & 1.4178 \\
\hline & & 25 & 1.7457 & 1.1346 & 1.0785 & 1.4249 & 1.2953 & 1.2447 \\
\hline & & 250 & 1.0849 & 1.0344 & 1.0627 & 1.0741 & 1.0310 & 1.0668 \\
\hline & \multirow{3}{*}{$\begin{array}{l}\text { High } \\
(\mathbf{8 5 \%})\end{array}$} & 10 & 2.4774 & 1.4218 & 1.2785 & 2.2692 & 2.0962 & 1.9228 \\
\hline & & 25 & 1.9757 & 1.3460 & 1.2330 & 1.8368 & 1.7190 & 1.6104 \\
\hline & & 250 & 1.1445 & 1.0813 & 1.0876 & 1.1221 & 1.0788 & 1.1026 \\
\hline
\end{tabular}




\subsection{2-way interaction tables}

\subsubsection{2-way DPLFM interaction tables}

The interactions between $C$ and $F R$

\begin{tabular}{|l|l|l|l|l}
\hline \multicolumn{2}{c|}{} & \multicolumn{3}{|l|}{ Forgetting rate $(F R)$} \\
\cline { 3 - 5 } \multicolumn{2}{c|}{} & $\mathbf{9 5 \%}$ & $\mathbf{9 0 \%}$ & $\mathbf{8 5 \%}$ \\
\hline \multirow{3}{*}{$\begin{array}{l}\text { Worker } \\
\text { transfer policy } \\
(C)\end{array}$} & $\mathbf{1 0}$ & 1.044 & 1.198 & 1.365 \\
\cline { 2 - 5 } & $\mathbf{2 5}$ & 1.020 & 1.080 & 1.181 \\
\cline { 2 - 5 } & $\mathbf{2 5 0}$ & 1.014 & 1.024 & 1.045 \\
\hline
\end{tabular}

The interactions between $C$ and $F A P$

\begin{tabular}{|l|l|l|l|l}
\hline \multicolumn{2}{c|}{} & \multicolumn{4}{|l}{ Upfront training policy $(F A P)$} \\
\cline { 3 - 5 } \multicolumn{2}{c|}{} & 0 & 1 & $\mathbf{2}$ \\
\hline \multirow{2}{*}{$\begin{array}{l}\text { Worker transfer } \\
\text { policy }(C)\end{array}$} & $\mathbf{1 0}$ & 1.514 & 1.053 & 1.040 \\
\cline { 2 - 5 } & $\mathbf{2 5}$ & 1.206 & 1.038 & 1.036 \\
\cline { 2 - 5 } & $\mathbf{2 5 0}$ & 1.032 & $\mathbf{1 . 0 2 0}$ & 1.031 \\
\hline
\end{tabular}

The interactions between $C$ and $N$

\begin{tabular}{|l|l|l|l|}
\hline \multicolumn{2}{|c|}{} & \multicolumn{2}{|l|}{ Number of tasks learned $(N)$} \\
\cline { 3 - 4 } \multicolumn{2}{|c|}{} & $\mathbf{2}$ & $\mathbf{3}$ \\
\hline \multirow{2}{*}{$\begin{array}{l}\text { Worker transfer } \\
\text { policy }(C)\end{array}$} & $\mathbf{1 0}$ & 1.138 & 1.267 \\
\cline { 2 - 4 } & $\mathbf{2 5}$ & 1.058 & 1.130 \\
\cline { 2 - 4 } & $\mathbf{2 5 0}$ & $\mathbf{1 . 0 2 1}$ & 1.034 \\
\hline
\end{tabular}

The interactions between $F R$ and $F A P$

\begin{tabular}{|l|l|l|l|l|}
\hline \multicolumn{2}{|c|}{} & \multicolumn{4}{|l|}{ Upfront training policy $(F A P)$} \\
\cline { 3 - 5 } \multicolumn{2}{c|}{} & 0 & 1 & 2 \\
\hline \multirow{2}{*}{$\begin{array}{l}\text { Forgetting rate } \\
(F R)\end{array}$} & $\mathbf{9 5 \%}$ & 1.056 & $\mathbf{1 . 0 0 3}$ & 1.019 \\
\cline { 2 - 5 } & $\mathbf{9 0 \%}$ & 1.255 & 1.019 & 1.028 \\
\cline { 2 - 6 } & $\mathbf{8 5 \%}$ & 1.441 & 1.089 & 1.060 \\
\hline
\end{tabular}


The interactions between $F R$ and $N$

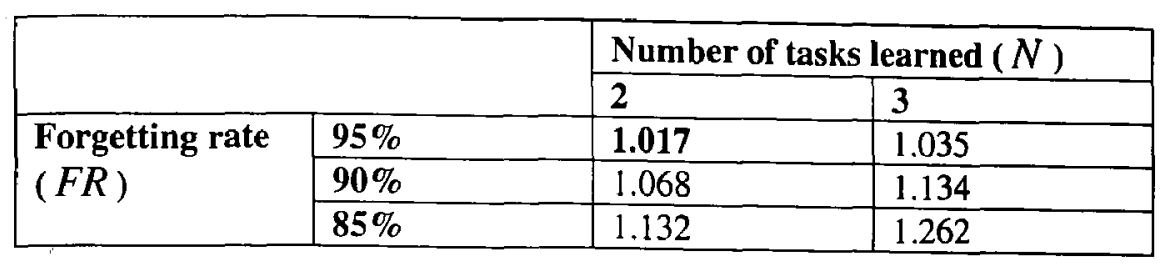

The interactions between $F A P$ and $N$

\begin{tabular}{|l|l|l|l|}
\hline \multicolumn{2}{|c|}{} & \multicolumn{2}{|l|}{ Number of tasks learned $(N)$} \\
\cline { 3 - 4 } \multicolumn{2}{|c|}{} & $\mathbf{2}$ & $\mathbf{3}$ \\
\hline $\begin{array}{l}\text { Upfront } \\
\text { training policy }\end{array}$ & $\mathbf{0}$ & 1.182 & 1.319 \\
\cline { 2 - 4 }$(F A P)$ & $\mathbf{1}$ & $\mathbf{1 . 0 1 1}$ & 1.063 \\
\cline { 2 - 4 } & $\mathbf{2}$ & 1.024 & 1.048 \\
\hline
\end{tabular}

The interactions between $C$ and $T$

\begin{tabular}{|l|l|l|l|l|l|}
\hline \multicolumn{2}{|c|}{} & \multicolumn{3}{|l|}{ Task-type $(T)$} \\
\cline { 3 - 7 } \multicolumn{2}{|c|}{} & $T_{C}$ & $T_{M}$ & $T_{\text {Med }}$ & $T_{U}$ \\
\hline $\begin{array}{l}\text { Worker } \\
\text { transfer } \\
\text { policy }(C)\end{array}$ & $\mathbf{1 0}$ & 1.126 & 1.301 & 1.202 & 1.180 \\
\cline { 2 - 6 } & $\mathbf{2 5}$ & 1.049 & 1.168 & 1.093 & 1.065 \\
\cline { 2 - 6 } & $\mathbf{2 5 0}$ & $\mathbf{1 . 0 1 8}$ & 1.046 & 1.025 & 1.021 \\
\hline
\end{tabular}

The interactions between $C$ and $R$

\begin{tabular}{|c|c|c|c|c|}
\hline & \multicolumn{3}{|c|}{ R-value $(R)$} \\
\hline & & $1 / 3$ & $\mathbf{1}$ & 3 \\
\hline \multirow{3}{*}{$\begin{array}{l}\text { Worker transfer } \\
\text { policy }(C)\end{array}$} & 10 & 1.280 & 1.206 & 1.121 \\
\hline & 25 & 1.146 & 1.089 & 1.045 \\
\hline & 250 & 1.039 & 1.025 & 1.018 \\
\hline
\end{tabular}

The interactions between $F R$ and $T$

\begin{tabular}{|l|l|l|l|l|l|}
\hline \multicolumn{2}{|c|}{} & \multicolumn{4}{|l|}{ Task-type $(T)$} \\
\cline { 3 - 6 } \multicolumn{2}{|c|}{} & $T_{C}$ & $T_{M}$ & $T_{\text {Med }}$ & $T_{U}$ \\
\hline \multirow{2}{*}{$\begin{array}{l}\text { Forgetting } \\
\text { rate }(F R)\end{array}$} & $\mathbf{9 5 \%}$ & $\mathbf{1 . 0 1 2}$ & 1.056 & 1.023 & 1.014 \\
\cline { 2 - 6 } & $\mathbf{9 0 \%}$ & 1.054 & 1.169 & 1.100 & 1.079 \\
\cline { 2 - 6 } & $\mathbf{8 5 \%}$ & 1.127 & 1.291 & 1.198 & 1.170 \\
\hline
\end{tabular}


The interactions between $F R$ and $R$

\begin{tabular}{|c|c|c|c|c|}
\hline & \multicolumn{3}{|c|}{ R-value $(R)$} \\
\hline & & $1 / 3$ & 1 & 3 \\
\hline \multirow{3}{*}{$\begin{array}{l}\text { Forgetting rate } \\
(F R)\end{array}$} & $95 \%$ & 1.046 & 1.021 & 1.011 \\
\hline & $90 \%$ & 1.153 & 1.096 & 1.052 \\
\hline & $85 \%$ & 1.267 & 1.203 & 1.121 \\
\hline
\end{tabular}

The interactions between $T$ and $F A P$

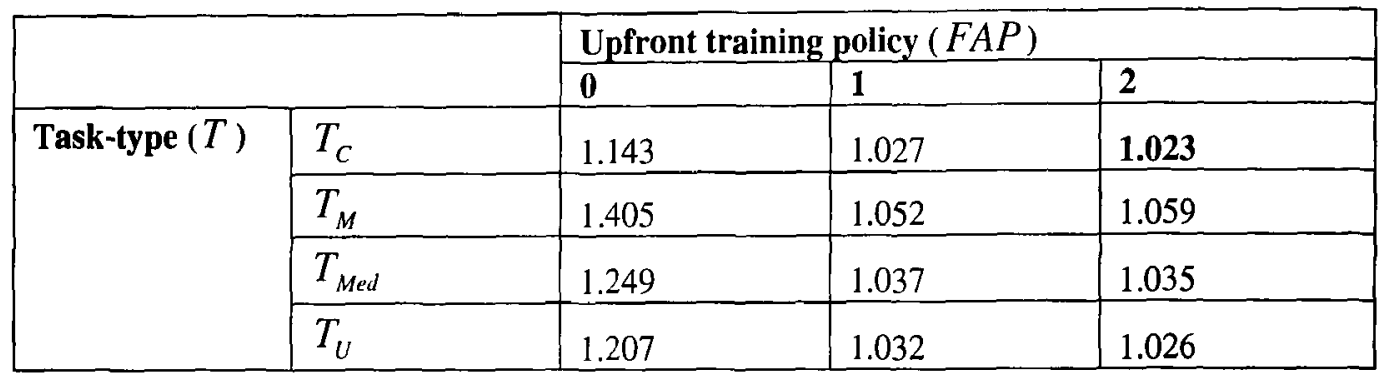

The interactions between $T$ and $N$

\begin{tabular}{|c|c|c|c|}
\hline & \multicolumn{2}{|c|}{ Number of tasks learned $(N)$} \\
\hline & & 2 & 3 \\
\hline \multirow[t]{4}{*}{ Task-type $(T)$} & $T_{C}$ & 1.039 & 1.090 \\
\hline & $T_{M}$ & 1.124 & 1.220 \\
\hline & $T_{\text {Med }}$ & 1.070 & 1.144 \\
\hline & $T_{U}$ & 1.056 & 1.121 \\
\hline
\end{tabular}

The interactions between $T$ and $R$

\begin{tabular}{|l|l|l|l|l|}
\hline \multicolumn{2}{|c|}{} & \multicolumn{2}{l|}{$\mathbf{R}$-value $(R)$} & $\mathbf{3}$ \\
\cline { 3 - 5 } Task-type $(T)$ & $T_{C}$ & 1.3 & 1 & 1.029 \\
\cline { 2 - 5 } & $T_{M}$ & 1.268 & 1.062 & 1.085 \\
\cline { 2 - 5 } & $T_{\text {Med }}$ & 1.170 & 1.101 & 1.049 \\
\cline { 2 - 5 } & $T_{U}$ & 1.082 & 1.073 & 1.073 \\
\hline
\end{tabular}


The interactions between $F A P$ and $R$

\begin{tabular}{|l|l|l|l|l|}
\hline \multicolumn{2}{|c|}{} & \multicolumn{2}{|l|}{$\mathbf{R}$-value $(R)$} & $\mathbf{1}$ \\
\cline { 3 - 5 } \multicolumn{2}{|c|}{$\mathbf{1 / 3}$} & $\mathbf{1}$ & $\mathbf{3}$ \\
\hline $\begin{array}{l}\text { Upfront } \\
\text { training policy } \\
(F A P)\end{array}$ & $\mathbf{0}$ & 1.364 & 1.249 & 1.139 \\
\cline { 2 - 5 } & $\mathbf{1}$ & 1.050 & 1.037 & 1.023 \\
\cline { 2 - 5 } & $\mathbf{2}$ & 1.052 & 1.034 & $\mathbf{1 . 0 2 2}$ \\
\hline
\end{tabular}

The interactions between $N$ and $R$

\begin{tabular}{|l|l|l|l|l|}
\hline \multicolumn{2}{|c|}{} & \multicolumn{3}{|l|}{ R-value $(R)$} \\
\cline { 3 - 5 } & $\mathbf{1 / 3}$ & $\mathbf{1}$ & $\mathbf{3}$ \\
\hline \multirow{2}{*}{$\begin{array}{l}\text { Number of tasks } \\
\text { learned }(N)\end{array}$} & $\mathbf{2}$ & 1.109 & 1.070 & $\mathbf{1 . 0 3 7}$ \\
\cline { 2 - 5 } & $\mathbf{3}$ & 1.201 & 1.144 & 1.086 \\
\hline
\end{tabular}

\subsubsection{2-way LFCM interaction tables}

The interactions between $C$ and $F R$

\begin{tabular}{|l|l|l|l|l|}
\hline \multicolumn{2}{|c|}{} & \multicolumn{3}{|l|}{ Forgetting rate $(F R)$} \\
\cline { 3 - 5 } \multicolumn{2}{|c|}{} & $\mathbf{9 5 \%}$ & $\mathbf{9 0 \%}$ & $\mathbf{8 5 \%}$ \\
\hline \multirow{2}{*}{$\begin{array}{l}\text { Worker } \\
\text { transfer policy } \\
(C)\end{array}$} & $\mathbf{1 0}$ & 1.185 & 1.406 & 1.579 \\
\cline { 2 - 5 } & $\mathbf{2 5}$ & 1.091 & 1.243 & 1.394 \\
\cline { 2 - 5 } & $\mathbf{2 5 0}$ & $\mathbf{1 . 0 3 2}$ & 1.050 & 1.081 \\
\hline
\end{tabular}

The interactions between $C$ and $F A P$

\begin{tabular}{|l|l|l|l|l|}
\hline \multicolumn{2}{|c|}{} & \multicolumn{3}{|l|}{ Upfront training policy (FAP) } \\
\cline { 3 - 5 } \multicolumn{2}{|c|}{} & $\mathbf{0}$ & $\mathbf{1}$ & $\mathbf{2}$ \\
\hline \multirow{3}{*}{$\begin{array}{l}\text { Worker transfer } \\
\text { policy }(C)\end{array}$} & $\mathbf{1 0}$ & 1.968 & 1.109 & 1.092 \\
\cline { 2 - 5 } & $\mathbf{2 5}$ & 1.542 & 1.100 & 1.087 \\
\cline { 2 - 5 } & $\mathbf{2 5 0}$ & 1.077 & $\mathbf{1 . 0 2 8}$ & 1.058 \\
\hline
\end{tabular}

The interactions between $C$ and $N$

\begin{tabular}{|l|l|l|l|}
\hline \multicolumn{2}{|c|}{} & \multicolumn{2}{|l|}{ Number of tasks learned $(N)$} \\
\cline { 3 - 4 } \multicolumn{2}{|c|}{$\mathbf{2}$} & $\mathbf{3}$ \\
\hline \multirow{2}{*}{$\begin{array}{l}\text { Worker transfer } \\
\text { policy }(C)\end{array}$} & $\mathbf{1 0}$ & 1.288 & 1.492 \\
\cline { 2 - 4 } & $\mathbf{2 5}$ & 1.168 & 1.317 \\
\cline { 2 - 4 } & $\mathbf{2 5 0}$ & $\mathbf{1 . 0 4 2}$ & 1.067 \\
\hline
\end{tabular}


The interactions between $F R$ and $F A P$

\begin{tabular}{|l|l|l|l|l|}
\hline \multicolumn{2}{|c|}{} & \multicolumn{3}{|l|}{ Upfront training policy $($ FAP) } \\
\cline { 3 - 5 } \multicolumn{2}{|c|}{} & $\mathbf{0}$ & $\mathbf{1}$ & $\mathbf{2}$ \\
\hline \multirow{3}{*}{$\begin{array}{l}\text { Forgetting rate } \\
(F R)\end{array}$} & $\mathbf{9 5 \%}$ & 1.246 & $\mathbf{1 . 0 1 5}$ & 1.047 \\
\cline { 2 - 5 } & $\mathbf{9 0 \%}$ & 1.576 & 1.061 & 1.062 \\
\cline { 2 - 5 } & $\mathbf{8 5 \%}$ & 1.765 & 1.161 & 1.128 \\
\hline
\end{tabular}

The interactions between $F R$ and $N$

\begin{tabular}{|l|l|l|l|}
\hline \multicolumn{2}{|c|}{} & \multicolumn{2}{|l|}{ Number of tasks learned $(N)$} \\
\cline { 3 - 4 } \multicolumn{2}{|c|}{} & $\mathbf{2}$ & $\mathbf{3}$ \\
\hline \multirow{3}{*}{$\begin{array}{l}\text { Forgetting rate } \\
(F R)\end{array}$} & $\mathbf{9 5 \%}$ & $\mathbf{1 . 0 7 1}$ & 1.134 \\
\cline { 2 - 4 } & $\mathbf{9 0 \%}$ & 1.774 & 1.292 \\
\cline { 2 - 4 } & $\mathbf{8 5 \%}$ & 1.253 & 1.450 \\
\hline
\end{tabular}

The interactions between $F A P$ and $N$

\begin{tabular}{|l|l|l|l|}
\hline \multicolumn{2}{|c|}{} & \multicolumn{2}{|l|}{ Number of tasks learned $(N)$} \\
\cline { 3 - 4 } \multicolumn{2}{c|}{} & $\mathbf{2}$ & $\mathbf{3}$ \\
\hline \multirow{2}{*}{$\begin{array}{l}\text { Upfront } \\
\text { training policy } \\
(F A P)\end{array}$} & $\mathbf{0}$ & 1.424 & 1.634 \\
\cline { 2 - 4 } & $\mathbf{1}$ & $\mathbf{1 . 0 2 4}$ & 1.134 \\
\cline { 2 - 4 } & $\mathbf{2}$ & 1.050 & 1.107 \\
\hline
\end{tabular}

\subsection{3-way interaction tables}

\subsubsection{3-way DPLFM interaction tables}

3-way interactions for $C_{10} F R_{85 \%}$

\begin{tabular}{|l|l|l|c|l|l|l|l|l|l|l|l|}
\hline \multicolumn{2}{|l|}{$C_{10} F R_{85 \%}$} & $\%$ & $F A P$ & & $\%$ & $N$ & & $\%$ & $R$ & & $\%$ \\
\hline$T$ & & $\%$ & & & & & & \\
\hline$T_{C}$ & 1.251 & $23.3 \%$ & 0 & 1.884 & $85.7 \%$ & 2 & 1.254 & $23.6 \%$ & $1 / 3$ & 1.442 & $42.1 \%$ \\
\hline$T_{M}$ & 1.493 & $47.2 \%$ & 1 & 1.135 & $11.9 \%$ & 3 & 1.476 & $45.5 \%$ & 1 & 1.376 & $35.7 \%$ \\
\hline$T_{\text {Med }}$ & 1.368 & $34.9 \%$ & 2 & 1.076 & $6.1 \%$ & & & & 3 & 1.302 & $28.3 \%$ \\
\hline$T_{U}$ & 1.346 & $32.7 \%$ & & & & & & & & & \\
\hline
\end{tabular}


3-way interactions for $C_{250} F R_{95 \%}$

\begin{tabular}{|l|r|r|r|l|l|l|l|l|l|l|l|}
\hline \multicolumn{2}{|l|}{$C_{250} F R_{95 \%}$} & \multicolumn{10}{l|}{} \\
\hline$T$ & & & $F A P$ & & $\%$ & $N$ & & $\%$ & $R$ & & $\%$ \\
\hline$T_{C}$ & 1.009 & $-0.5 \%$ & 0 & 1.019 & $0.5 \%$ & 2 & 1.013 & $-0.1 \%$ & 3 & 1.070 & $5.5 \%$ \\
\hline$T_{M}$ & 1.025 & $1.0 \%$ & 1 & 1.003 & $-1.1 \%$ & 3 & 1.015 & $0.1 \%$ & 1 & 1.013 & $-0.1 \%$ \\
\hline$T_{M e d}$ & 1.013 & $-0.1 \%$ & 2 & 1.021 & $0.7 \%$ & & & & 3 & 1.009 & $-0.5 \%$ \\
\hline$T_{U}$ & 1.018 & $0.4 \%$ & & & & & & & & & \\
\hline
\end{tabular}

3-way interactions for $C_{10} F A P-0$

\begin{tabular}{|c|c|c|c|c|c|c|c|c|c|c|c|}
\hline \multicolumn{12}{|c|}{$C_{10} F A P-0$} \\
\hline$F R$ & & $\%$ & $T$ & & $\%$ & $N$ & & $\%$ & $R$ & & $\%$ \\
\hline $95 \%$ & 1.412 & $9.0 \%$ & $T_{C}$ & 1.317 & $29.1 \%$ & 2 & 1.383 & $35.6 \%$ & $1 / 3$ & 1.675 & $64.2 \%$ \\
\hline $90 \%$ & 1.547 & $51.7 \%$ & $T_{M}$ & 1.764 & $73.0 \%$ & 3 & 1.645 & $61.3 \%$ & 1 & 1.525 & $49.3 \%$ \\
\hline \multirow[t]{2}{*}{$85 \%$} & 1.884 & $84.7 \%$ & $T_{\text {Med }}$ & 1.513 & $48.4 \%$ & & & & 3 & 1.379 & $35.2 \%$ \\
\hline & & & $T_{U}$ & 1.462 & $43.4 \%$ & & & & & & \\
\hline
\end{tabular}

3-way interactions for $C_{250} F A P-1$

\begin{tabular}{|l|l|l|l|l|l|l|l|l|l|l|l|}
\hline \multicolumn{2}{|c|}{$C_{250} F A P-1$} & $\%$ & $T$ & & $\%$ & $N$ & & $\%$ & $R$ & & $\%$ \\
\hline$F R$ & & $\%$ & & & & & & \\
\hline $95 \%$ & 1.003 & $-1.7 \%$ & $T_{C}$ & 1.017 & $-0.3 \%$ & 2 & 1.012 & $-0.7 \%$ & $1 / 3$ & 1.027 & $0.7 \%$ \\
\hline $90 \%$ & 1.015 & $-0.5 \%$ & $T_{M}$ & 1.025 & $0.5 \%$ & 3 & 1.028 & $0.7 \%$ & 1 & 1.026 & $0.6 \%$ \\
\hline $85 \%$ & 1.042 & $2.1 \%$ & $T_{\text {Med }}$ & 1.020 & $0.0 \%$ & & & & 3 & 1.026 & $0.6 \%$ \\
\hline & & & $T_{U}$ & 1.018 & $-0.2 \%$ & & & & & & \\
\hline
\end{tabular}


3-way interactions for $C_{10} N_{3}$

\begin{tabular}{|c|c|c|c|c|c|c|c|c|c|c|c|}
\hline \multicolumn{12}{|c|}{$C_{10} N_{3}$} \\
\hline$F R$ & & $\%$ & $T$ & & $\%$ & $F A P$ & & $\%$ & $R$ & & $\%$ \\
\hline $95 \%$ & 1.064 & $4.2 \%$ & $T_{C}$ & 1.176 & $15.1 \%$ & 0 & 1.645 & $61.1 \%$ & $1 / 3$ & 1.358 & $32.9 \%$ \\
\hline $90 \%$ & 1.260 & $23.4 \%$ & $T_{M}$ & 1.380 & $35.2 \%$ & 1 & 1.097 & $7.4 \%$ & 1 & 1.274 & $24.7 \%$ \\
\hline \multirow[t]{2}{*}{$85 \%$} & 1.476 & $44.5 \%$ & $T_{M e d}$ & 1.270 & $24.4 \%$ & 2 & 1.059 & $3.6 \%$ & 3 & 1.169 & $14.5 \%$ \\
\hline & & & $T_{U}$ & 1.240 & $21.4 \%$ & & & & & & \\
\hline
\end{tabular}

3-way interactions for $\mathrm{C}_{250} \mathrm{~N}_{2}$

\begin{tabular}{|l|l|l|r|l|l|r|r|r|r|r|r|}
\hline \multicolumn{2}{|l|}{$C_{250} N_{2}$} \\
\hline$F R$ & & $\%$ & $T$ & & $\%$ & $F A P$ & & $\%$ & $R$ & & $\%$ \\
\hline $95 \%$ & 1.013 & $-0.8 \%$ & $T_{C}$ & 1.014 & $-0.7 \%$ & 0 & 1.026 & $0.4 \%$ & $1 / 3$ & 1.031 & $0.9 \%$ \\
\hline $90 \%$ & 1.019 & $-0.2 \%$ & $T_{M}$ & 1.035 & $1.4 \%$ & 1 & 1.012 & $-0.9 \%$ & 1 & 1.020 & $-0.2 \%$ \\
\hline $85 \%$ & 1.032 & $1.1 \%$ & $T_{\text {Med }}$ & 1.020 & $-0.2 \%$ & 2 & 1.026 & $0.5 \%$ & 3 & 1.014 & $-0.7 \%$ \\
\hline & & & $T_{U}$ & 1.016 & $-0.5 \%$ & & & & & & \\
\hline
\end{tabular}

3-way interactions for $F R_{85 \%} F A P-0$

\begin{tabular}{|c|c|c|c|c|c|c|c|c|c|c|c|}
\hline \multicolumn{12}{|c|}{$F R_{85 \%} F A P-0$} \\
\hline$C$ & & $\%$ & $T$ & & $\%$ & $N$ & & $\%$ & $R$ & & $\%$ \\
\hline 10 & 1.884 & $87.9 \%$ & $T_{C}$ & 1.279 & $27.5 \%$ & 2 & 1.341 & $33.8 \%$ & $1 / 3$ & 1.592 & $58.8 \%$ \\
\hline 25 & 1.390 & $38.7 \%$ & $T_{M}$ & 1.648 & $64.4 \%$ & 3 & 1.540 & $53.6 \%$ & 1 & 1.457 & $45.3 \%$ \\
\hline 256 & 17048 & $4.6 \%$ & $T_{\text {Med }}$ & 1.441 & $43.7 \%$ & & & & 3 & 1.274 & $27.0 \%$ \\
\hline & & & $T_{U}$ & 1.395 & $39.2 \%$ & & & & & & \\
\hline
\end{tabular}


3-way interactions for $F R_{95 \%} F A P-1$

\begin{tabular}{|c|c|c|c|c|c|c|c|c|c|c|c|}
\hline \multicolumn{12}{|c|}{$F R_{95 \%} F A P-1$} \\
\hline$C$ & & $\%$ & $T$ & & $\%$ & $N$ & & $\%$ & $R$ & & $\%$ \\
\hline 10 & 1.002 & $-0.1 \%$ & $T_{c}$ & 1.003 & $0.0 \%$ & 2 & 1.001 & $-0.1 \%$ & $1 / 3$ & 1.003 & $0.0 \%$ \\
\hline 25 & 1.003 & $0.0 \%$ & $T_{M}$ & 1.003 & $0.0 \%$ & 3 & 1.004 & $0.1 \%$ & 1 & 1.003 & $0.0 \%$ \\
\hline 250 & 1.003 & $0.0 \%$ & $T_{\text {Med }}$ & 1.003 & $0.0 \%$ & & & & 3 & 1.003 & $0.0 \%$ \\
\hline & & & $T_{U}$ & 1.003 & $0.0 \%$ & & & & & & \\
\hline
\end{tabular}

3-way interactions for $F R_{85 \%} N_{3}$

\begin{tabular}{|c|c|c|c|c|c|c|c|c|c|c|c|}
\hline \multicolumn{12}{|c|}{$F R_{85 \%} N_{3}$} \\
\hline$T$ & & $\%$ & $F A P$ & & $\%$ & $R$ & & $\%$ & $\bar{C}$ & & $\%$ \\
\hline$T_{C}$ & 1.176 & $15.7 \%$ & 0 & 1.540 & $51.4 \%$ & $1 / 3$ & 1.346 & $32.3 \%$ & 10 & 1.476 & $45.1 \%$ \\
\hline$T_{M}$ & 1.372 & $34.9 \%$ & 1 & 1.154 & $13.5 \%$ & 1 & 1.271 & $25.0 \%$ & 25 & 1.252 & $23.1 \%$ \\
\hline$T_{\text {Med }}$ & 1.264 & $24.3 \%$ & 2 & 1.090 & $7.2 \%$ & 3 & 1.168 & $14.8 \%$ & 250 & 1.057 & $3.9 \%$ \\
\hline$T_{U}$ & 1.234 & $21.3 \%$ & & & & & & & & & \\
\hline
\end{tabular}

3-way interactions for $F R_{95 \%} N_{2}$

\begin{tabular}{|c|c|c|c|c|c|c|c|c|c|c|c|}
\hline \multicolumn{12}{|c|}{$F R_{95 \%} N_{2}$} \\
\hline$T$ & & $\%$ & $F A P$ & & $\%$ & $R$ & & $\%$ & $\bar{C}$ & & $\%$ \\
\hline$T_{C}$ & 1.009 & $-0.8 \%$ & 0 & 1.031 & $1.4 \%$ & $1 / 3$ & 1.029 & $1.2 \%$ & 10 & 1.024 & $0.6 \%$ \\
\hline$T_{M}$ & 1.036 & $1.8 \%$ & 1 & 1.001 & $-1.5 \%$ & 1 & 1.013 & $-0.4 \%$ & 25 & 1.014 & $-0.3 \%$ \\
\hline$T_{\text {Med }}$ & 1.013 & $-0.4 \%$ & 2 & 1.018 & $0.1 \%$ & 3 & 1.009 & $-0.8 \%$ & 250 & 1.013 & $-0.4 \%$ \\
\hline$T_{U}$ & 1.011 & $-0.6 \%$ & & & & & & & & & \\
\hline
\end{tabular}


3-way interactions for $\mathrm{FAP}-\mathrm{ON}_{3}$

\begin{tabular}{|c|c|c|c|c|c|c|c|c|c|c|c|}
\hline \multicolumn{12}{|c|}{$F A P-0 N_{3}$} \\
\hline$C$ & & $\%$ & $F R$ & & $\%$ & $T$ & & $\%$ & $R$ & & $\%$ \\
\hline 10 & 1.645 & $62.8 \%$ & $95 \%$ & 1.081 & $7.0 \%$ & $T_{C}$ & 1.195 & $18.3 \%$ & $1 / 3$ & 1.447 & $43.1 \%$ \\
\hline 25 & 1.275 & $26.1 \%$ & $90 \%$ & 1.336 & $32.2 \%$ & $T_{M}$ & 1.490 & $47.4 \%$ & 1 & 1.320 & $30.6 \%$ \\
\hline 250 & 1,038 & $2.7 \%$ & $85 \%$ & 1.540 & $52.4 \%$ & $T_{\text {Med }}$ & 1.318 & $30.4 \%$ & 3 & 1.191 & $17.8 \%$ \\
\hline & & & & & & $T_{U}$ & 1.274 & $26.0 \%$ & & & \\
\hline
\end{tabular}

3-way interactions for $F A P-1 N_{2}$

\begin{tabular}{|c|c|c|c|c|c|c|c|c|c|c|c|}
\hline \multicolumn{12}{|c|}{$F A P-1 N_{2}$} \\
\hline$C$ & & $\%$ & $F R$ & & $\%$ & $T$ & & $\%$ & $R$ & & $\%$ \\
\hline 10 & 1.008 & $-0.2 \%$ & $95 \%$ & 1.001 & $-0.9 \%$ & $T_{C}$ & 1.010 & $-0.1 \%$ & $1 / 3$ & 1.014 & $0.3 \%$ \\
\hline 25 & 1.012 & $0.1 \%$ & $90 \%$ & 1.007 & $-0.4 \%$ & $T_{M}$ & 1.014 & $0.3 \%$ & 1 & 1.010 & $-0.1 \%$ \\
\hline 250 & 1.012 & $0.1 \%$ & $85 \%$ & 1.024 & $1.3 \%$ & $T_{\text {Med }}$ & 1.009 & $-0.1 \%$ & 3 & 1.009 & $-0.2 \%$ \\
\hline & & & & & & $T_{U}$ & 1.010 & $-0.1 \%$ & & & \\
\hline
\end{tabular}

3-way interactions for $C_{10} T_{M}$

\begin{tabular}{|c|r|r|r|r|r|r|r|r|r|r|r|}
\hline \multicolumn{2}{|c|}{$C_{10} T_{M}$} \\
\hline$F R$ & & $\%$ & $F A P$ & & $\%$ & $N$ & & $\%$ & $R$ & & $\%$ \\
\hline $95 \%$ & 1.101 & $8.1 \%$ & 0 & 1.764 & $73.2 \%$ & 2 & 1.222 & $20.0 \%$ & $1 / 3$ & 1.443 & $41.7 \%$ \\
\hline $90 \%$ & 1.310 & $28.7 \%$ & 1 & 1.074 & $5.4 \%$ & 3 & 1.380 & $35.6 \%$ & 1 & 1.293 & $27.0 \%$ \\
\hline $85 \%$ & 1.493 & $46.6 \%$ & 2 & 1.067 & $4.7 \%$ & & & & 3 & 1.169 & $14.8 \%$ \\
\hline & & & & & & & & & & & \\
\hline
\end{tabular}

3-way interactions for $C_{250} T_{C}$

\begin{tabular}{|c|r|r|r|r|r|r|r|r|r|r|r|}
\hline \multicolumn{10}{|l|}{$C_{250} T_{C}$} \\
\hline$F R$ & & $\%$ & $F A P$ & & $\%$ & $N$ & & $\%$ & $R$ & & $\%$ \\
\hline $95 \%$ & 1.009 & $-0.9 \%$ & 0 & 1.016 & $-0.2 \%$ & 2 & 1.014 & $-0.4 \%$ & $1 / 3$ & 1.023 & $0.5 \%$ \\
\hline $90 \%$ & 1.016 & $-0.2 \%$ & 1 & 1.017 & $-0.1 \%$ & 3 & 1.022 & $0.4 \%$ & 1 & 1.018 & $0.0 \%$ \\
\hline $85 \%$ & 1.030 & $1.2 \%$ & 2 & 1.022 & $0.3 \%$ & & & & 3 & 1.014 & $-0.5 \%$ \\
\hline & & & & & & & & & & & \\
\hline
\end{tabular}


3-way interactions for $C_{10} R_{1 / 3}$

\begin{tabular}{|c|c|c|c|c|c|c|c|c|c|c|c|}
\hline \multicolumn{12}{|c|}{$C_{10} R_{1 / 3}$} \\
\hline$F R$ & & $\%$ & $T$ & & $\%$ & $F A P$ & & $\%$ & $N$ & & $\%$ \\
\hline $95 \%$ & 1.084 & $6.5 \%$ & $T_{C}$ & 1.200 & $17.9 \%$ & 0 & 1.707 & $67.8 \%$ & 2 & 1.202 & $18.1 \%$ \\
\hline $90 \%$ & 1.289 & $26.6 \%$ & $T_{M}$ & 1.443 & $41.7 \%$ & 1 & 1072 & 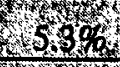 & 3 & 1.358 & $33.4 \%$ \\
\hline \multirow[t]{2}{*}{$85 \%$} & 1.466 & $44.1 \%$ & $T_{\text {Med }}$ & 1.309 & $28.6 \%$ & 2 & $\begin{array}{l}1.059 \\
1.28\end{array}$ & 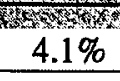 & & & \\
\hline & & & $T_{U}$ & 1.167 & $14.7 \%$ & & & & & & \\
\hline
\end{tabular}

3-way interactions for $C_{250} R_{3}$

\begin{tabular}{|c|c|c|c|c|r|r|r|r|r|r|r|}
\hline \multicolumn{10}{|c|}{$C_{250} R_{3}$} & \multicolumn{10}{|c|}{$\%$} & $T$ & & $\%$ & $F A P$ & & $\%$ & $N$ & & $\%$ \\
\hline$F R$ & & & & & & & & & \\
\hline $95 \%$ & 1.009 & $-0.9 \%$ & $T_{C}$ & 1.014 & $-0.4 \%$ & 0 & 1.017 & $-0.1 \%$ & 2 & 1.014 & $-0.4 \%$ \\
\hline $90 \%$ & 1.016 & $-0.2 \%$ & $T_{M}$ & 1.022 & $0.4 \%$ & 1 & 1.016 & $-0.2 \%$ & 3 & 1.022 & $0.4 \%$ \\
\hline $85 \%$ & 1.029 & $1.0 \%$ & $T_{\text {Med }}$ & 1.016 & $-0.2 \%$ & 2 & 1.021 & $0.3 \%$ & & & \\
\hline & & & $T_{U}$ & 1.020 & $0.2 \%$ & & & & & & \\
\hline
\end{tabular}

3-way interactions for $F R_{85 \%} T_{M}$

\begin{tabular}{|r|r|r|r|r|r|r|r|r|r|r|r|}
\hline \multicolumn{2}{|l|}{$F R_{85 \%} T_{M}$} & \multicolumn{10}{|l|}{$\%$} \\
\hline$C$ & & $\%$ & $F A P$ & & $\%$ & $N$ & & $\%$ & $R$ & & $\%$ \\
\hline 10 & 1.493 & $47.5 \%$ & 0 & 1.648 & $62.8 \%$ & 2 & 1.209 & $19.5 \%$ & $1 / 3$ & 1.419 & $40.2 \%$ \\
\hline 25 & 1.304 & $28.8 \%$ & 1 & 1.126 & $11.2 \%$ & 3 & 1.372 & $35.6 \%$ & 1 & 1.287 & $27.2 \%$ \\
\hline 250 & 1.075 & $6.2 \%$ & 2 & 1.098 & $8.5 \%$ & & & & 3 & 1.165 & $15.2 \%$ \\
\hline & & & & & & & & & & & \\
\hline
\end{tabular}

3-way interactions for $F R_{95 \%} T_{C}$

\begin{tabular}{|r|r|r|r|r|r|r|r|r|r|r|r|}
\hline \multicolumn{2}{|l|}{$F R_{95 \%} T_{C}$} & \multicolumn{10}{l|}{$\%$} \\
\hline$C$ & & $\%$ & $F A P$ & & & $N$ & & & $R$ & & $\%$ \\
\hline 10 & 1.017 & $0.5 \%$ & 0 & 1.022 & $0.9 \%$ & 2 & 1.009 & $-0.3 \%$ & $1 / 3$ & 1.019 & $0.6 \%$ \\
\hline 25 & 1.011 & $-0.1 \%$ & 1 & 1.003 & $-0.9 \%$ & 3 & 1.016 & $0.3 \%$ & 1 & 1.010 & $-0.2 \%$ \\
\hline 250 & 1.009 & $-0.3 \%$ & 2 & 1.012 & $0.0 \%$ & & & & 3 & 1.008 & $-0.4 \%$ \\
\hline & & & & & & & & & & & \\
\hline
\end{tabular}


3-way interactions for $F R_{85 \%} R_{1 / 3}$

\begin{tabular}{|c|c|c|c|c|c|c|c|c|c|c|c|}
\hline \multicolumn{12}{|c|}{$F R_{85 \%} R_{1 / 3}$} \\
\hline$C$ & & $\%$ & $T$ & & $\%$ & $F A P$ & & $\%$ & $N$ & & $\%$ \\
\hline 10 & 1.466 & $45.0 \%$ & $T_{C}$ & 1.191 & $17.8 \%$ & 0 & 1.592 & $57.4 \%$ & 2 & 1.187 & $17.4 \%$ \\
\hline 25 & 1.268 & $25.4 \%$ & $T_{M}$ & 1.419 & $40.4 \%$ & $2 x$ & 120 & $10 \% \div \%$ & 3 & 1.346 & $33.1 \%$ \\
\hline 250 & 1.065 & $5.3 \%$ & $T_{\text {Med }}$ & 1.297 & $28.3 \%$ & \% & $\begin{array}{l}1.088 \\
\end{array}$ & $7.6 \%$ & & & \\
\hline & & & $T_{U}$ & 1.159 & $14.6 \%$ & & & & & & \\
\hline
\end{tabular}

3-way interactions for $F R_{95 F_{\%}} R_{3}$

\begin{tabular}{|c|c|c|c|c|c|c|c|c|c|c|c|}
\hline \multicolumn{12}{|c|}{$F R_{95 \%} R_{3}$} \\
\hline$C$ & & $\%$ & $T$ & & $\%$ & $F A P$ & & $\%$ & $N$ & & $\%$ \\
\hline 10 & 1.013 & $0.2 \%$ & $T_{C}$ & 1.008 & $-0.3 \%$ & 0 & 1.018 & $0.7 \%$ & 2 & 1.009 & $-0.2 \%$ \\
\hline 25 & 1.011 & $0.0 \%$ & $T_{M}$ & 1.014 & $0.3 \%$ & 1 & 1.003 & $-0.8 \%$ & 3 & 1.013 & $0.2 \%$ \\
\hline 250 & 1.009 & $-0.2 \%$ & $T_{\text {Med }}$ & 1.010 & $-0.1 \%$ & 2 & 1.013 & $0.1 \%$ & & & \\
\hline & & & $T_{u}$ & 1.013 & $0.2 \%$ & & & & & & \\
\hline
\end{tabular}

3-way interactions for $T_{M} F A P-0$

\begin{tabular}{|l|r|r|r|l|l|r|r|r|r|r|r|}
\hline \multicolumn{2}{|l|}{$T_{M} F A P-0$} \\
\hline$C$ & & $\%$ & $F R$ & & $\%$ & $N$ & & $\%$ & $R$ & & $\%$ \\
\hline 10 & 1.764 & $72.4 \%$ & $95 \%$ & 1.131 & $10.5 \%$ & 2 & 1.319 & $28.9 \%$ & $1 / 3$ & 1.628 & $59.1 \%$ \\
\hline 25 & 1.386 & $35.4 \%$ & $90 \%$ & 1.435 & $40.2 \%$ & 3 & 1.490 & $45.7 \%$ & 1 & 1.384 & $35.3 \%$ \\
\hline 2 & & & & & & & & & & & \\
\hline
\end{tabular}

3-way interactions for $T_{C} F A P-2$

\begin{tabular}{|r|r|r|r|r|r|r|r|r|r|r|r|}
\hline \multicolumn{2}{|l|}{$T_{C}$ FAP-2 } \\
\hline \multicolumn{1}{l|}{$C$} & & $\%$ & $F R$ & & $\%$ & $N$ & & $\%$ & $R$ & & $\%$ \\
\hline 10 & 1.024 & $0.1 \%$ & $95 \%$ & 1.012 & $-1.1 \%$ & 2 & 1.016 & $-0.7 \%$ & $1 / 3$ & 1.030 & $0.7 \%$ \\
\hline 25 & 1.023 & $0.0 \%$ & $90 \%$ & 1.019 & $-0.4 \%$ & 3 & 1.030 & $0.7 \%$ & 1 & 1.022 & $-0.1 \%$ \\
\hline 250 & 1.022 & $-0.2 \%$ & $85 \%$ & 1.038 & $1.4 \%$ & & & & 3 & 1.017 & $-0.6 \%$ \\
\hline & & & & & & & & & & & \\
\hline
\end{tabular}


3-way interactions for $T_{M} N_{3}$

\begin{tabular}{|c|c|c|c|c|c|c|c|c|c|c|c|}
\hline \multicolumn{12}{|c|}{$T_{M} N_{3}$} \\
\hline$C$ & & $\%$ & $F R$ & & $\%$ & $F A P$ & & $\%$ & $R$ & & $\%$ \\
\hline 10 & 1.380 & $32.9 \%$ & $95 \%$ & 1.075 & $3.5 \%$ & 0 & 1.490 & $43.5 \%$ & $1 / 3$ & 1.331 & $28.1 \%$ \\
\hline 25 & 1.222 & $17.6 \%$ & $90 \%$ & 1.212 & $16.7 \%$ & 1 & 1.090 & $4.9 \%$ & 1 & 1.212 & $16.7 \%$ \\
\hline 280 & 105 & $1.7 \%$ & $85 \%$ & 1.372 & $32.1 \%$ & 482 & 1079 & $309 \%$ & 3 & 1.117 & $7.5 \%$ \\
\hline & & & & & & & & & & & \\
\hline
\end{tabular}

3-way interactions for $T_{C} N_{2}$

\begin{tabular}{|l|r|r|r|r|r|r|r|r|r|r|r|}
\hline \multicolumn{10}{|l|}{$T_{C} N_{2}$} \\
\hline \multicolumn{1}{l|}{$C$} & & $\%$ & $F R$ & & $\%$ & $F A P$ & & $\%$ & $R$ & & $\%$ \\
\hline 10 & 1.075 & $3.5 \%$ & $95 \%$ & 1.009 & $-2.9 \%$ & 0 & 1.090 & $4.9 \%$ & $1 / 3$ & 1.064 & $2.5 \%$ \\
\hline 25 & 1.027 & $-1.2 \%$ & $90 \%$ & 1.030 & $-0.8 \%$ & 1 & 1.010 & $-2.8 \%$ & 1 & 1.036 & $-0.2 \%$ \\
\hline 250 & 1.014 & $-2.3 \%$ & $85 \%$ & 1.077 & $3.7 \%$ & 2 & 1.016 & $-2.2 \%$ & 3 & 1.015 & $-2.2 \%$ \\
\hline & & & & & & & & & & & \\
\hline
\end{tabular}

3-way interactions for $T_{M} R_{1 / 3}$

\begin{tabular}{|c|c|c|c|c|c|c|c|c|c|c|c|}
\hline \multicolumn{12}{|c|}{$T_{M} R_{1 / 3}$} \\
\hline$C$ & & $\%$ & $F R$ & & $\%$ & $F A P$ & & $\%$ & $N$ & & $\%$ \\
\hline 10 & 1.443 & $40.1 \%$ & $95 \%$ & 1.110 & $7.9 \%$ & 0 & 1.628 & $58.2 \%$ & 2 & 1.206 & $17.1 \%$ \\
\hline 25 & 1.282 & $24.5 \%$ & $90 \%$ & 1.275 & $23.8 \%$ & 1 & 1.080 & $4.9 \%$ & 3 & 1.331 & $29.3 \%$ \\
\hline 繁 & 11080 & $4.9 \%$ & $85 \%$ & 1.419 & $37.9 \%$ & 2 & 1.096 & $6.5 \%$ & & & \\
\hline & & & & & & & & & & & \\
\hline
\end{tabular}

3-way interactions for $T_{C} R_{3}$

\begin{tabular}{|r|r|r|r|r|r|r|r|r|r|r|r|}
\hline \multicolumn{2}{|l|}{$T_{C} R_{3}$} & \multicolumn{10}{|c|}{$\%$} \\
\hline$C$ & & $\%$ & $F R$ & & $\%$ & $F A P$ & & $\%$ & $N$ & & $\%$ \\
\hline 10 & 1.050 & $2.0 \%$ & $95 \%$ & 1.008 & $-2.1 \%$ & 0 & 1.053 & $2.3 \%$ & 2 & 1.015 & $-1.4 \%$ \\
\hline 25 & 1.024 & $-0.5 \%$ & $90 \%$ & 1.020 & $-0.9 \%$ & 1 & 1.017 & $-1.2 \%$ & 3 & 1.043 & $1.4 \%$ \\
\hline 250 & 1.014 & $-1.5 \%$ & $85 \%$ & 1.060 & $3.0 \%$ & 2 & 1.017 & $-1.2 \%$ & & & \\
\hline & & & & & & & & & & & \\
\hline
\end{tabular}


3-way interactions for $F A P-O R_{1 / 3}$

\begin{tabular}{|c|c|c|c|c|c|c|c|c|c|c|c|}
\hline \multicolumn{11}{|c|}{$F A P-0 R_{1 / 3}$} & $3^{2}$ \\
\hline$C$ & & $\%$ & $F R$ & & $\%$ & $T$ & & $\%$ & $N$ & & $\%$ \\
\hline 10 & 1.707 & $67.1 \%$ & $95 \%$ & 1.108 & $8.4 \%$ & $T_{C}$ & 1.237 & $21.0 \%$ & 2 & 1.281 & $25.4 \%$ \\
\hline 25 & 1.333 & $30.4 \%$ & $90 \%$ & 1.393 & $36.3 \%$ & $T_{M}$ & 1.628 & $59.3 \%$ & 3 & 1.447 & $41.6 \%$ \\
\hline 250 & 1.052 & $3.0 \%$ & $85 \%$ & 1.592 & $55.8 \%$ & $T_{\text {Med }}$ & 1.399 & $36.9 \%$ & & & \\
\hline & & & & & & $T_{U}$ & 1.192 & $16.7 \%$ & & & \\
\hline
\end{tabular}

3-way interactions for $F A P-2 R_{3}$

\begin{tabular}{|r|r|r|r|l|r|r|r|r|r|r|r|}
\hline \multicolumn{2}{|l|}{$F A P-2 R_{3}$} & $\%$ & $F R$ & & $\%$ & $T$ & & $\%$ & $N$ & & $\%$ \\
\hline$C$ & & & & & & & & \\
\hline 10 & 1.022 & $0.0 \%$ & $95 \%$ & 1.013 & $-0.9 \%$ & $T_{C}$ & 1.017 & $-0.5 \%$ & 2 & 1.016 & $-0.6 \%$ \\
\hline 25 & 1.023 & $0.1 \%$ & $90 \%$ & 1.019 & $-0.3 \%$ & $T_{M}$ & 1.027 & $0.4 \%$ & 3 & 1.028 & $0.6 \%$ \\
\hline 250 & 1.021 & $-0.1 \%$ & $85 \%$ & 1.034 & $1.2 \%$ & $T_{\text {Med }}$ & 1.020 & $-0.2 \%$ & & & \\
\hline & & & & & & $T_{U}$ & 1.024 & $0.2 \%$ & & & \\
\hline
\end{tabular}

3-way interactions for $N_{3} R_{1 / 3}$

\begin{tabular}{|c|c|c|c|c|c|c|c|c|c|c|c|}
\hline \multicolumn{12}{|c|}{$N_{3} R_{1 / 3}$} \\
\hline$C$ & & $\%$ & $F R$ & & $\%$ & $T$ & & $\%$ & $F A P$ & & $\%$ \\
\hline 10 & 1.358 & $30.9 \%$ & $95 \%$ & 1.063 & $25 \%$ & $T_{C}$ & 1.138 & $9.7 \%$ & 0 & 1.447 & $39.5 \%$ \\
\hline 25 & 1.197 & $15.4 \%$ & $90 \%$ & 1.194 & $15.1 \%$ & $T_{M}$ & 1.331 & $28.3 \%$ & 1 & 1.086 & $4.7 \%$ \\
\hline 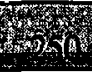 & W & $10 \%$ & $85 \%$ & 1.346 & $29.8 \%$ & $T_{\text {Med }}$ & 1.224 & $18.0 \%$ & 2 & $10 \mathrm{OM}$ & 48 \\
\hline & & & & & & $T_{U}$ & 1.112 & $7.2 \%$ & & & \\
\hline
\end{tabular}


3-way interactions for $\mathrm{N}_{2} R_{3}$

\begin{tabular}{|c|c|c|c|c|c|c|c|c|c|c|c|}
\hline \multicolumn{12}{|c|}{$N_{2} R_{3}$} \\
\hline$C$ & & $\%$ & $F R$ & & $\%$ & $T$ & & $\%$ & $F A P$ & & $\%$ \\
\hline 10 & 1.074 & $3.5 \%$ & $95 \%$ & 1.009 & $-2.7 \%$ & $T_{c}$ & 1.015 & $-2.1 \%$ & 0 & 1.087 & $4.8 \%$ \\
\hline 25 & 1.024 & $-1.3 \%$ & $90 \%$ & 1.029 & $-0.8 \%$ & $T_{M}$ & 1.054 & $1.6 \%$ & 1 & 1.009 & $-2.7 \%$ \\
\hline 250 & 4014 & $22 \%$ & $85 \%$ & 1.074 & $3.5 \%$ & $T_{M e d}$ & 1.028 & $-0.9 \%$ & $6+7$ & & 80 \\
\hline & & & & & & $T_{U}$ & 1.051 & $1.4 \%$ & & & \\
\hline
\end{tabular}

\subsubsection{3-way LFCM interaction tables}

3-way interactions for $C_{10} F R_{85 \%}$

\begin{tabular}{|l|r|r|r|r|r|}
\hline \multicolumn{6}{|l|}{$C_{10} F R_{85 \%}$} \\
\hline$N$ & & $\%$ & $F A P$ & & $\%$ \\
\hline 2 & 1.431 & $38.8 \%$ & 0 & 2.335 & $126.3 \%$ \\
\hline 3 & 1.726 & $67.3 \%$ & 1 & 1.235 & $19.7 \%$ \\
\hline & & & 2 & 1.166 & $13.0 \%$ \\
\hline
\end{tabular}

3-way interactions for $C_{250} F R_{95 \%}$

\begin{tabular}{|r|r|r|r|r|r|}
\hline \multicolumn{2}{|c|}{$C_{250} F R_{95 \%}$} \\
\hline$N$ & & $\%$ & $F A P$ & & \multicolumn{1}{l|}{} \\
\hline 2 & 1.030 & $-0.2 \%$ & 0 & 1.047 & $1.4 \%$ \\
\hline 3 & 1.035 & $0.3 \%$ & 1 & 1.005 & $-2.6 \%$ \\
\hline & & & 2 & 1.045 & $1.3 \%$ \\
\hline
\end{tabular}

3-way interactions for $C_{10} F A P-0$

\begin{tabular}{|r|r|r|r|r|r|}
\hline \multicolumn{6}{|c|}{$C_{10} F A P-0$} \\
\hline$N$ & & $\%$ & $F R$ & & $\%$ \\
\hline 2 & 1.800 & $75.0 \%$ & $95 \%$ & 1.511 & $46.9 \%$ \\
\hline 3 & 2.137 & $107.8 \%$ & $90 \%$ & 2.059 & $100.3 \%$ \\
\hline & & & $85 \%$ & 2.335 & $127.1 \%$ \\
\hline
\end{tabular}


3-way interactions for $C_{250} F A P-1$

\begin{tabular}{|r|r|r|r|r|r|}
\hline \multicolumn{2}{|c|}{$C_{250} F A P-1$} \\
\hline$N$ & & $\%$ & $F R$ & & $\%$ \\
\hline 2 & 1.016 & $-1.2 \%$ & $95 \%$ & 1.005 & $-2.2 \%$ \\
\hline 3 & 1.041 & $1.2 \%$ & $90 \%$ & 1.023 & $-0.5 \%$ \\
\hline & & & $85 \%$ & 1.056 & $2.7 \%$ \\
\hline
\end{tabular}

3-way interactions for $C_{10} N_{3}$

\begin{tabular}{|c|c|c|c|c|c|}
\hline \multicolumn{6}{|c|}{$\mathrm{C}_{10} \mathrm{~N}_{3}$} \\
\hline FAP & & $\%$ & $F R$ & & $\%$ \\
\hline 0 & 2.137 & $105 \%$ & $95 \%$ & 1.253 & $20.2 \%$ \\
\hline 6 & $\overline{11}$ & $15.1 \%$ & $90 \%$ & 1.496 & $43.5 \%$ \\
\hline 852 & 1139 & $9.2 \%$ & $85 \%$ & 1.726 & $65.6 \%$ \\
\hline
\end{tabular}

3-way interactions for $\mathrm{C}_{250} \mathrm{~N}_{2}$

\begin{tabular}{|r|r|r|r|r|r|}
\hline \multicolumn{2}{|l|}{$C_{250} N_{2}$} & \multicolumn{3}{l|}{} \\
\hline$F A P$ & & $\%$ & $F R$ & & $\%$ \\
\hline 0 & 1.060 & $1.7 \%$ & $95 \%$ & 1.030 & $-1.2 \%$ \\
\hline 1 & 1.016 & $-2.6 \%$ & $90 \%$ & 1.039 & $-0.3 \%$ \\
\hline 2 & 1.051 & $0.8 \%$ & $85 \%$ & 1.057 & $1.5 \%$ \\
\hline
\end{tabular}

3-way interactions for $F R_{85 \%} F A P-0$

\begin{tabular}{|r|r|r|r|r|r|}
\hline \multicolumn{2}{|c|}{$F R_{85 \%} F A P-0$} \\
\hline$N$ & & $\%$ & $C$ & & \multicolumn{1}{l|}{} \\
\hline 2 & 1.664 & $64.0 \%$ & 10 & 2.335 & $130.1 \%$ \\
\hline 3 & 1.866 & $83.9 \%$ & 25 & 1.847 & $82.0 \%$ \\
\hline & & & 250 & 1.113 & $9.7 \%$ \\
\hline
\end{tabular}

3-way interactions for $F R_{95 \%} F A P-1$

\begin{tabular}{|l|r|r|r|r|r|}
\hline \multicolumn{6}{|l|}{$F R_{95 \%} F A P-1$} \\
\hline$N$ & & $\%$ & $C$ & & $\%$ \\
\hline 2 & 1.024 & $0.9 \%$ & 10 & 1.003 & $-1.2 \%$ \\
\hline 3 & 1.006 & $-0.9 \%$ & 25 & 1.036 & $2.1 \%$ \\
\hline & & & 250 & 1.005 & $-0.9 \%$ \\
\hline
\end{tabular}


3-way interactions for $\mathrm{FR}_{85 \%} \mathrm{~N}_{3}$

\begin{tabular}{|r|r|r|r|r|l|}
\hline \multicolumn{2}{|l|}{$F R_{85 \%} N_{3}$} \\
\hline$F A P$ & & $\%$ & $C$ & & $\%$ \\
\hline 0 & 1.866 & $74.2 \%$ & 10 & 1.726 & $61.1 \%$ \\
\hline 1 & 1.283 & $19.8 \%$ & 25 & 1.518 & $41.8 \%$ \\
\hline 2 & 1.200 & $12.0 \%$ & 250 & 1.10 & 20 \\
\hline
\end{tabular}

3-way interactions for $F R_{95 \%} N_{2}$

\begin{tabular}{|r|r|r|r|r|r|}
\hline \multicolumn{6}{|l|}{$\mathrm{FR}_{95 \%} \mathrm{~N}_{2}$} \\
\hline FAP & & $\%$ & \multicolumn{1}{l|}{$\mathrm{C}$} & & $\%$ \\
\hline 0 & 1.139 & $6.4 \%$ & 10 & 1.116 & $4.2 \%$ \\
\hline 1 & 1.024 & $-4.4 \%$ & 25 & 1.068 & $-0.3 \%$ \\
\hline 2 & 1.050 & $-2.0 \%$ & 250 & 1.030 & $-3.9 \%$ \\
\hline
\end{tabular}

3-way interactions for $\mathrm{FAP}-0 \mathrm{~N}_{3}$

\begin{tabular}{|c|c|c|c|c|c|}
\hline \multicolumn{6}{|c|}{$F A P-0 N_{3}$} \\
\hline$F R$ & & $\%$ & $C$ & & $\%$ \\
\hline $95 \%$ & 1.353 & $32.2 \%$ & 10 & 2.137 & $108.7 \%$ \\
\hline $90 \%$ & 1.683 & $64.4 \%$ & 25 & 1.672 & $63.3 \%$ \\
\hline $85 \%$ & 1.866 & $82.2 \%$ & 250 & 1.093 & $68.8 \%$ \\
\hline
\end{tabular}

3-way interactions for $F A P-1 N_{2}$

\begin{tabular}{|r|r|r|r|r|r|}
\hline \multicolumn{5}{|c|}{$F A P-1 N_{2}$} \\
\hline$F R$ & & $\%$ & $C$ & & $\%$ \\
\hline $95 \%$ & 1.024 & $0.0 \%$ & 10 & 1.019 & $-0.5 \%$ \\
\hline $90 \%$ & 1.009 & $-1.5 \%$ & 25 & 1.038 & $1.3 \%$ \\
\hline $85 \%$ & 1.039 & $1.5 \%$ & 250 & 1.016 & $-0.8 \%$ \\
\hline
\end{tabular}




\subsection{Suppressed model A results - APT (DPLFM)}

$$
N=2, F R=95 \%
$$

\begin{tabular}{|c|c|c|c|c|c|}
\hline$C$ & Task Type & $R$ & $F A P-0$ & $F A P-1$ & $F A P-2$ \\
\hline \multirow{12}{*}{10} & \multirow{3}{*}{$T_{C}$} & $1 / 3$ & - & - & - \\
\hline & & 1 & - & - & - \\
\hline & & 3 & - & - & - \\
\hline & \multirow{3}{*}{$T_{M}$} & $1 / 3$ & 1.3609 & - & - \\
\hline & & 1 & - & - & - \\
\hline & & 3 & - & - & - \\
\hline & \multirow{3}{*}{$T_{\text {Med }}$} & $1 / 3$ & - & - & - \\
\hline & & 1 & - & - & - \\
\hline & & 3 & - & - & - \\
\hline & \multirow{3}{*}{$T_{U}$} & $1 / 3$ & - & - & - \\
\hline & & 1 & - & - & - \\
\hline & & 3 & - & - & - \\
\hline \multirow{11}{*}{25} & \multirow{3}{*}{$T_{C}$} & $1 / 3$ & - & - & - \\
\hline & & 1 & - & - & - \\
\hline & & 3 & - & - & - \\
\hline & \multirow{3}{*}{$T_{M}$} & $1 / 3$ & 1.0867 & - & - \\
\hline & & 1 & - & - & - \\
\hline & & 3 & - & - & - \\
\hline & \multirow{2}{*}{$T_{\text {Med }}$} & $1 / 3$ & - & - & - \\
\hline & & 3 & - & - & - \\
\hline & \multirow{3}{*}{$T_{u}$} & $1 / 3$ & - & - & - \\
\hline & & 1 & - & - & - \\
\hline & & 3 & - & - & - \\
\hline \multirow{12}{*}{250} & \multirow{3}{*}{$T_{C}$} & $1 / 3$ & - & - & - \\
\hline & & 1 & - & - & - \\
\hline & & 3 & - & - & - \\
\hline & \multirow{3}{*}{$T_{M}$} & $1 / 3$ & - & - & - \\
\hline & & 1 & - & - & - \\
\hline & & 3 & $=$ & - & - \\
\hline & \multirow{3}{*}{$T_{\text {Med }}$} & $1 / 3$ & - & - & - \\
\hline & & 1 & - & $=$ & - \\
\hline & & 3 & - & - & - \\
\hline & \multirow{3}{*}{$T_{u}$} & $1 / 3$ & - & - & - \\
\hline & & 1 & - & - & - \\
\hline & & 3 & - & - & - \\
\hline
\end{tabular}




$$
N=2, F R=90 \%
$$

\begin{tabular}{|c|c|c|c|c|c|}
\hline$C$ & Task Type & $R$ & $F A P-0$ & $F A P-1$ & $F A P-2$ \\
\hline \multirow{12}{*}{10} & \multirow{3}{*}{$T_{C}$} & $1 / 3$ & 1.3784 & - & - \\
\hline & & 1 & 1.0814 & - & - \\
\hline & & 3 & - & - & - \\
\hline & \multirow{3}{*}{$T_{M}$} & $1 / 3$ & 2.1088 & - & - \\
\hline & & 1 & 1.6664 & - & - \\
\hline & & 3 & 1.2797 & - & - \\
\hline & \multirow{3}{*}{$T_{\text {Med }}$} & $1 / 3$ & 1.7298 & - & - \\
\hline & & 1 & 1.3600 & - & - \\
\hline & & 3 & - & - & - \\
\hline & \multirow{3}{*}{$T_{U}$} & $1 / 3$ & 1.2673 & - & - \\
\hline & & 1 & 1.3750 & - & - \\
\hline & & 3 & 1.2673 & - & - \\
\hline \multirow{11}{*}{25} & \multirow{3}{*}{$T_{C}$} & $1 / 3$ & - & - & - \\
\hline & & 1 & - & - & $=$ \\
\hline & & $\frac{3}{1 / 3}$ & $\frac{-}{16000}$ & - & - \\
\hline & \multirow[t]{2}{*}{$T_{M}$} & 1 & 1.2155 & - & - \\
\hline & & 3 & - & - & - \\
\hline & \multirow{3}{*}{$T_{\text {Med }}$} & $1 / 3$ & 1.2672 & - & - \\
\hline & & 1 & - & - & - \\
\hline & & 3 & - & - & - \\
\hline & \multirow{3}{*}{$T_{U}$} & $1 / 3$ & - & - & - \\
\hline & & 1 & - & - & - \\
\hline & & 3 & - & - & - \\
\hline \multirow{12}{*}{250} & \multirow{3}{*}{$T_{C}$} & $1 / 3$ & - & - & - \\
\hline & & 1 & - & - & - \\
\hline & & 3 & - & - & - \\
\hline & \multirow{3}{*}{$T_{M}$} & $1 / 3$ & 1.0760 & - & - \\
\hline & & 1 & - & - & - \\
\hline & & 3 & - & - & - \\
\hline & \multirow{3}{*}{$T_{\text {Med }}$} & $1 / 3$ & - & - & - \\
\hline & & 1 & - & - & - \\
\hline & & 3 & - & - & - \\
\hline & \multirow{3}{*}{$T_{U}$} & $1 / 3$ & - & - & - \\
\hline & & 1 & - & - & - \\
\hline & & 3 & - & - & - \\
\hline
\end{tabular}


$N=2, F R=85 \%$

\begin{tabular}{|c|c|c|c|c|c|}
\hline C & Task Type & $R$ & $F A P-0$ & $F A P-1$ & $F A P-2$ \\
\hline \multirow{12}{*}{10} & \multirow{3}{*}{$T_{C}$} & $1 / 3$ & 1.7251 & - & - \\
\hline & & 1 & 1.4919 & - & - \\
\hline & & 3 & 1.0835 & - & - \\
\hline & \multirow{3}{*}{$T_{M}$} & $1 / 3$ & 2.3914 & 1.0869 & 1.0805 \\
\hline & & 1 & 2.0386 & - & - \\
\hline & & 3 & 1.6370 & - & - \\
\hline & \multirow{3}{*}{$T_{\text {Med }}$} & $1 / 3$ & 2.0511 & - & - \\
\hline & & 1 & 1.7541 & - & - \\
\hline & & 3 & 1.3595 & - & - \\
\hline & \multirow{3}{*}{$T_{U}$} & $1 / 3$ & 1.6324 & - & - \\
\hline & & 1 & 1.7746 & - & - \\
\hline & & 3 & 1.6324 & - & - \\
\hline \multirow{12}{*}{25} & \multirow{3}{*}{$T_{C}$} & $1 / 3$ & 1.2281 & - & - \\
\hline & & 1 & 1.0735 & - & - \\
\hline & & 3 & - & - & - \\
\hline & \multirow{3}{*}{$T_{M}$} & $1 / 3$ & 1.9010 & 1.0785 & 1.0794 \\
\hline & & 1 & 1.5409 & - & - \\
\hline & & 3 & 1.1716 & - & - \\
\hline & \multirow{3}{*}{$T_{\text {Med }}$} & $1 / 3$ & 1.5471 & - & - \\
\hline & & 1 & 1.2574 & - & - \\
\hline & & 3 & - & - & - \\
\hline & \multirow{3}{*}{$T_{U}$} & $1 / 3$ & 1.1227 & - & - \\
\hline & & 1 & 1.2250 & - & - \\
\hline & & 3 & 1.1227 & - & - \\
\hline \multirow{12}{*}{250} & \multirow{3}{*}{$T_{C}$} & $1 / 3$ & - & - & - \\
\hline & & 1 & - & - & - \\
\hline & & 3 & - & - & - \\
\hline & \multirow{3}{*}{$T_{M}$} & $1 / 3$ & 1.1506 & - & 1.0826 \\
\hline & & 1 & - & - & - \\
\hline & & 3 & - & - & - \\
\hline & \multirow{3}{*}{$T_{\text {Med }}$} & $1 / 3$ & - & - & - \\
\hline & & 1 & - & $=$ & - \\
\hline & & 3 & - & - & - \\
\hline & \multirow{3}{*}{$T_{U}$} & $1 / 3$ & - & - & - \\
\hline & & 1 & - & - & - \\
\hline & & 3 & 二 & - & - \\
\hline
\end{tabular}


$N=3, F R=95 \%$

\begin{tabular}{|c|c|c|c|c|c|}
\hline$C$ & Task Type & $R$ & $F A P-0$ & $F A P-1$ & $F A P-2$ \\
\hline \multirow{12}{*}{10} & \multirow{3}{*}{$T_{C}$} & $1 / 3$ & 1.1263 & - & - \\
\hline & & 1 & - & - & - \\
\hline & & 3 & - & - & - \\
\hline & \multirow{3}{*}{$T_{M}$} & $1 / 3$ & 1.7488 & - & - \\
\hline & & 1 & 1.3887 & - & - \\
\hline & & 3 & - & - & - \\
\hline & \multirow{3}{*}{$T_{\text {Med }}$} & $1 / 3$ & 1.4156 & - & - \\
\hline & & 1 & 1.0811 & - & - \\
\hline & & 3 & . & - & - \\
\hline & \multirow{3}{*}{$T_{U}$} & $1 / \mathbf{3}$ & - & - & - \\
\hline & & 1 & 1.0850 & - & - \\
\hline & & 3 & - &. & - \\
\hline \multirow{12}{*}{25} & \multirow{3}{*}{$T_{C}$} & $1 / 3$ & - & - & - \\
\hline & & 1 & - & - & - \\
\hline & & 3 & - & - & - \\
\hline & \multirow{3}{*}{$T_{M}$} & $1 / 3$ & 1.3159 & $\therefore$ & - \\
\hline & & 1 & - & - & - \\
\hline & & 3 & - & - & - \\
\hline & \multirow{3}{*}{$T_{\text {Med }}$} & $1 / 3$ & - & - & - \\
\hline & & 1 & - & - & - \\
\hline & & 3 & - & - & - \\
\hline & \multirow{3}{*}{$T_{U}$} & $1 / 3$ & - & - & - \\
\hline & & 1 & - & - & - \\
\hline & & 3 & - & - & - \\
\hline \multirow{12}{*}{250} & \multirow{3}{*}{$T_{C}$} & $1 / 3$ & - & - & - \\
\hline & & 1 & $\therefore$ & $=$ & - \\
\hline & & 3 & - & - & - \\
\hline & \multirow{3}{*}{$T_{M}$} & $1 / 3$ & - & - & - \\
\hline & & 1 & - & - & - \\
\hline & & 3 & $=$ & - & - \\
\hline & \multirow{3}{*}{$T_{\text {Med }}$} & $1 / 3$ & - & - & - \\
\hline & & 1 & - & - & - \\
\hline & & 3 & - & - & - \\
\hline & \multirow{3}{*}{$T_{U}$} & $1 / 3$ & - & - & - \\
\hline & & 1 & - & - & - \\
\hline & & 3 & - & - & - \\
\hline
\end{tabular}


$N=3, F R=90 \%$

\begin{tabular}{|c|c|c|c|c|c|}
\hline$C$ & Task Type & $R$ & $F A P-0$ & $F A P-1$ & $F A P-2$ \\
\hline \multirow{12}{*}{10} & \multirow{3}{*}{$T_{C}$} & $1 / 3$ & 1.7420 & - & $\ldots$ \\
\hline & & 1 & 1.4647 & - & - \\
\hline & & 3 & 1.0833 & - & - \\
\hline & \multirow{3}{*}{$T_{M}$} & $1 / 3$ & 2.3971 & 1.1351 & 1.1079 \\
\hline & & 1 & 2.0008 & - & - \\
\hline & & 3 & 1.6476 & - & - \\
\hline & \multirow{3}{*}{$T_{\text {Med }}$} & $1 / 3$ & 2.0616 & 1.0919 & - \\
\hline & & 1 & 1.7204 & - & - \\
\hline & & 3 & 1.3773 & - & - \\
\hline & \multirow{3}{*}{$T_{U}$} & $1 / 3$ & 1.6480 & - & - \\
\hline & & 1 & 1.7472 & - & - \\
\hline & & 3 & 1.6480 & - & - \\
\hline \multirow{12}{*}{25} & \multirow{3}{*}{$T_{C}$} & $1 / 3$ & 1.2385 & - & - \\
\hline & & 1 & - & - & - \\
\hline & & 3 & - & - & - \\
\hline & \multirow{3}{*}{$T_{M}$} & $1 / 3$ & 1.8715 & 1.1131 & 1.0990 \\
\hline & & 1 & 1.5121 & - & - \\
\hline & & 3 & 1.1711 & - & - \\
\hline & \multirow{3}{*}{$T_{\text {Med }}$} & $1 / 3$ & 1.5616 & - & - \\
\hline & & 1 & 1.2253 & - & - \\
\hline & & 3 & - & - & - \\
\hline & \multirow{3}{*}{$T_{U}$} & $1 / 3$ & 1.1251 & - & - \\
\hline & & 1 & 1.1932 & - & - \\
\hline & & 3 & 1.1251 & - & - \\
\hline \multirow{12}{*}{250} & \multirow{3}{*}{$T_{C}$} & $1 / 3$ & - & - & - \\
\hline & & 1 & - & - & - \\
\hline & & 3 & - & - & - \\
\hline & \multirow{3}{*}{$T_{M}$} & $1 / 3$ & 1.1191 & - & 1.0798 \\
\hline & & 1 & $=$ & - & - \\
\hline & & 3 & - & - & - \\
\hline & \multirow{3}{*}{$T_{\text {Med }}$} & $1 / 3$ & - & - & - \\
\hline & & 1 & - & - & - \\
\hline & & 3 & - & - & - \\
\hline & \multirow{3}{*}{$T_{u}$} & $1 / 3$ & - & - & - \\
\hline & & 1 & - & - & - \\
\hline & & 3 & - & - & - \\
\hline
\end{tabular}




$$
N=3, F R=85 \%
$$

\begin{tabular}{|c|c|c|c|c|c|}
\hline$C$ & Task Type & $R$ & $F A P-0$ & $F A P-1$ & $F A P-2$ \\
\hline \multirow{12}{*}{10} & \multirow{3}{*}{$T_{C}$} & $1 / 3$ & 2.0664 & 1.2708 & 1.1274 \\
\hline & & 1 & 1.8559 & 1.1903 & - \\
\hline & & 3 & 1.4711 & - & - \\
\hline & \multirow{3}{*}{$T_{M}$} & $1 / 3$ & 2.6494 & 1.4259 & 1.3012 \\
\hline & & 1 & 2.3408 & 1.3416 & 1.2275 \\
\hline & & 3 & 1.9818 & 1.2181 & 1.0805 \\
\hline & \multirow{3}{*}{$T_{\text {Med }}$} & $1 / 3$ & 2.3549 & 1.3814 & 1.2599 \\
\hline & & 1 & 2.0906 & 1.2697 & 1.1301 \\
\hline & & 3 & 1.7287 & 1.1005 & - \\
\hline & \multirow{3}{*}{$T_{U}$} & $1 / 3$ & 1.9866 & 1.2271 & - \\
\hline & & 1 & 2.1198 & 1.2937 & 1.1291 \\
\hline & & 3 & 1.9866 & 1.2271 & - \\
\hline \multirow{12}{*}{25} & \multirow{3}{*}{$T_{C}$} & $1 / 3$ & 1.5078 & 1.1533 & - \\
\hline & & 1 & 1.2545 & 1.0828 & - \\
\hline & & 3 & 1.0870 & - & - \\
\hline & \multirow{3}{*}{$T_{M}$} & $1 / 3$ & 2.1302 & 1.3605 & 1.2634 \\
\hline & & 1 & 1.8015 & 1.2623 & 1.1718 \\
\hline & & 3 & 1.4555 & 1.1062 & - \\
\hline & \multirow{3}{*}{$T_{\text {Med }}$} & $1 / 3$ & 1.8090 & 1.2912 & 1.1936 \\
\hline & & 1 & 1.5335 & 1.1612 & - \\
\hline & & 3 & 1.1561 & - & - \\
\hline & \multirow{3}{*}{$T_{U}$} & $1 / 3$ & 1.4220 & 1.0879 & - \\
\hline & & 1 & 1.5065 & 1.1561 & - \\
\hline & & 3 & 1.4220 & 1.0879 & $=$ \\
\hline \multirow{12}{*}{250} & \multirow{3}{*}{$T_{C}$} & $1 / 3$ & - & - & - \\
\hline & & 1 & - & - & - \\
\hline & & 3 & - & - & - \\
\hline & \multirow{3}{*}{$T_{M}$} & $1 / 3$ & 1.2789 & 1.1234 & 1.1253 \\
\hline & & 1 & 1.0836 & - & 1.0702 \\
\hline & & 3 & - & - & - \\
\hline & \multirow{3}{*}{$T_{\text {Med }}$} & $1 / 3$ & 1.0818 & 1.0711 & 1.0717 \\
\hline & & 1 & - & - & - \\
\hline & & 3 & - & - & $=$ \\
\hline & \multirow{3}{*}{$T_{U}$} & $1 / 3$ & - & - & - \\
\hline & & 1 & - & - & - \\
\hline & & 3 & - & - & - \\
\hline
\end{tabular}




\subsection{Model B results $(A P T)$}

\subsubsection{Two tasks learned}

Suppressed average processing time results for the DPLFM with the task similarity factor $-C=10, N=2$

\begin{tabular}{|c|c|c|c|c|c|c|c|c|c|c|c|}
\hline \multirow{2}{*}{$\begin{array}{l} \\
\text { Task- } \\
\text { Type }\end{array}$} & \multirow[b]{2}{*}{$R$} & \multirow[b]{2}{*}{$\begin{array}{l}\text { Similarity } \\
\text { Factor } \\
(S)\end{array}$} & \multicolumn{3}{|c|}{$\begin{array}{l}\text { Low forgetting } \\
\text { rate of } 95 \%\end{array}$} & \multicolumn{3}{|c|}{$\begin{array}{l}\text { Medium forgetting } \\
\text { rate of } 90 \%\end{array}$} & \multicolumn{3}{|c|}{$\begin{array}{l}\text { High forgetting } \\
\text { rate of } 85 \%\end{array}$} \\
\hline & & & $\begin{array}{c}\text { FAP } \\
0\end{array}$ & $\begin{array}{c}F A P \\
1\end{array}$ & $\begin{array}{c}F A P \\
2\end{array}$ & $\begin{array}{c}F A P \\
0\end{array}$ & $\begin{array}{c}F A P \\
1\end{array}$ & $\begin{array}{c}F A P \\
2\end{array}$ & $\begin{array}{c}F A P \\
0\end{array}$ & $\begin{array}{c}\text { FAP } \\
1\end{array}$ & $\begin{array}{c}\text { FAP } \\
2\end{array}$ \\
\hline \multirow{15}{*}{$T_{C}$} & \multirow{5}{*}{$1 / 3$} & 0.0 & $\therefore$ &. & - & 1.3784 & - & - & 1.7251 & - & $\therefore$ \\
\hline & & 0.2 & $\cdot$ & - & - & 1.2539 & - & - & 1.6014 & - & - \\
\hline & & 0.4 & - & - & - & 1.1057 & - & - & 1.4475 & - & - \\
\hline & & 0.6 & - & - & - &. & - & - & 1.2467 & - & - \\
\hline & & 0.8 & $\therefore$ & - & $\therefore$ & - & - & $\therefore$ & - & - & - \\
\hline & \multirow{5}{*}{1} & 0.0 & - & $\dot{-}$ & - & 1.0814 & - & - & 1.4919 & - & - \\
\hline & & 0.2 & - & - & - & - & - & - & 1.3661 & - & - \\
\hline & & 0.4 & $\therefore$ & - & - & + & - & - & $1.2 \mathrm{~J} 22$ & - & - \\
\hline & & 0.6 & - & - & 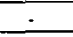 & - & - & - & - & - & - \\
\hline & & 0.8 & - & - & - & - & - & - & - & - & - \\
\hline & \multirow{5}{*}{3} & 0.0 & $\therefore$ & - & $\therefore$ & - & - & - & 1.0835 & - & $=$ \\
\hline & & 0.2 &. & - & - & - & - & $\cdot$ & - & - & - \\
\hline & & 0.4 & - & $\therefore$ & $\therefore$ & - & . & - & - & - & - \\
\hline & & 0.6 & $\therefore$ & $=$ & - & $\cdot$ & - & - & - & - & - \\
\hline & & 0.8 & $\cdot$ & - & - & - & - & - & - & - & - \\
\hline \multirow{15}{*}{$T_{M}$} & \multirow{5}{*}{$1 / 3$} & 0.0 & 1.3509 & - & - & 2.1088 & - & - & 2.3914 & 1.0869 & 1.0805 \\
\hline & & 0.2 & 1.2489 & - & - & 1.9974 & - & - & 2.2876 & - & - \\
\hline & & 0.4 & 1.1366 & - & - & 1.8574 & - & - & 2.1541 & - & - \\
\hline & & 0.6 & 1.0742 & - & - & 1.6716 & $=$ & - & 1.9708 & - & - \\
\hline & & 0.8 &. & - & - & 1.3994 & - & - & 1.6822 & - & $=$ \\
\hline & & 0.0 & - & - & - & 1.6664 & - & - & 2.0386 & - & - \\
\hline & & 0.2 & $\therefore$ & $\therefore$ & - & 1.5466 & - & - & 1.9239 & - & - \\
\hline & & 0.4 & - & 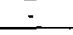 & $\therefore$ & 1.4013 & - & - & 1.7793 & - & - \\
\hline & & 0.6 & - & - & - & 1.2201 & . & $\cdot$ & 1.5864 & - & - \\
\hline & & 0.8 &. & - & - & - & - & - & 1.2994 & - & - \\
\hline & & 0.0 & 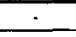 & $=$ & - & 1.2797 & - & - & 1.6370 & - & - \\
\hline & & 0.2 & - & - & I & 1.1580 & - & - & 15137 & - & - \\
\hline & & 0.4 & - & - & - & - & - & - & 1.3621 & - & - \\
\hline & & 0.6 & . & - & - & - & - & - & 1.1679 & - & - \\
\hline & & 0.8 & - & - & - & - & - & - & $\cdot$ & - & - \\
\hline & & 0.0 & $\therefore$ & $\cdot$ & $=$ & 1.7298 & - & - & $2.05 \mathrm{II}$ & - & - \\
\hline & & 0.2 & . & - & - & 1.6087 & - & - & 1.9353 & - & - \\
\hline & $1 / 3$ & 0.4 & - & - & $=$ & 1.4597 & - & - & 1.7886 & $\therefore$ & - \\
\hline & & 0.6 & $\therefore$ & - & $\therefore$ & 1.2699 & - & - & 1.5918 & - & - \\
\hline & & 0.8 & 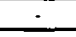 & - & $=$ & - & $=$ & - & 1.2957 & - & - \\
\hline & & 0.0 & - & - & - & 1.3600 & - & - & 1.7541 & - & - \\
\hline & & 0.2 & $\therefore$ & - & - & 1.2380 &. & - & 1.6322 & - & - \\
\hline$I_{\text {Med }}$ & & 0.4 & - & - & 5 & 1.0940 & - & - & 1.4806 & - & - \\
\hline & & 0.6 & $\therefore$ & $\dot{-}$ & - & - & - & - & 1.2827 & - & - \\
\hline & & 0.8 & $\dot{-}$ & - & - & - & - & 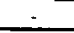 & - & - & - \\
\hline & & 0.0 & - & - & - & - & $\therefore$ & - & 1,3595 & - & - \\
\hline & & 0.2 & $\therefore$ & - & - & - & - & - & 1.2341 & $\therefore$ & - \\
\hline & 3 & 0.4 & - & - & - & - & - & - & 1.0717 & - & - \\
\hline & & 0.6 & - & - & - & - &. & - & - & - & - \\
\hline & & 0.8 & . & - & - & - & - & - & - & - & - \\
\hline & & 0.0 & - & - & - & 1.2673 & $\therefore$ & - & 1.6324 & - & - \\
\hline & & 0.2 & - & $=$ & - & 1.1393 & - & - & 1.5047 & - & - \\
\hline & $1 / 3$ & 0.4 & $\cdot$ & - & $\therefore$ & - & - & - & 1.3464 & - & - \\
\hline & & 0.6 & - & - & \pm & $\therefore$ & - & - & 1.1414 & - & 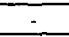 \\
\hline & & 0.8 & $\therefore$ & - & $=$ & . & $\therefore$ & - & $\cdot$ & - & - \\
\hline & & 0.0 & - & - & - & 1.3750 & - & $=$ & 1.7746 & - & - \\
\hline & & 0.2 & . & - & - & 1.2463 & $=$ & . & $1.64 \overline{83}$ & - & $=$ \\
\hline$I_{U}$ & & 0.4 & $=$ & - & - & 1.0920 &. & - & 1.4896 & - & $\therefore$ \\
\hline & & 0.6 & - & $=$ & - & - &. & - & 1.2793 & - & - \\
\hline & & 0.8 & - & - & - & - & - & $\therefore$ & - & - & $\therefore$ \\
\hline & & 0.0 & $\therefore$ & + & - & 1.2673 & 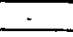 &. & 1.6324 & - & $=$ \\
\hline & & 0.2 & $\therefore$ & $\therefore$ & $=$ & 1.1393 & - & - & 1.5047 & - & - \\
\hline & & 0.4 & - & - & $\therefore$ & - & $=$ & - & 1.3464 & - & $\cdot$ \\
\hline & & 0.6 & - & - & $=$ & - & - & - & 1.1414 & - & - \\
\hline & & 0.8 & - & - & $\therefore$ & - & - & - & - & - & - \\
\hline
\end{tabular}


Suppressed average processing time results for the DPLFM with the task similarity factor $-C=25, N=2$

\begin{tabular}{|c|c|c|c|c|c|c|c|c|c|c|c|}
\hline \multirow[b]{2}{*}{$\begin{array}{l}\text { Task- } \\
\text { Type }\end{array}$} & \multirow[b]{2}{*}{$R$} & \multirow[b]{2}{*}{$\begin{array}{l}\text { Similarity } \\
\text { Factor }(S)\end{array}$} & \multicolumn{3}{|c|}{$\begin{array}{l}\text { Low forgetting } \\
\text { rate of } 95 \%\end{array}$} & \multicolumn{3}{|c|}{$\begin{array}{l}\text { Medium forgetting } \\
\text { rate of } 90 \%\end{array}$} & \multicolumn{3}{|c|}{$\begin{array}{l}\text { High forgetting } \\
\text { rate of } 85 \%\end{array}$} \\
\hline & & & $\begin{array}{c}F A P \\
0\end{array}$ & $\begin{array}{c}F A P \\
1\end{array}$ & $\begin{array}{c}F A P \\
2\end{array}$ & $\begin{array}{c}F A P \\
0\end{array}$ & $\begin{array}{c}F A P \\
1\end{array}$ & $\begin{array}{c}F A P \\
2\end{array}$ & $\begin{array}{c}\text { FAP } \\
0\end{array}$ & $\begin{array}{c}\text { FAP } \\
1\end{array}$ & $\begin{array}{c}\text { FAP } \\
2\end{array}$ \\
\hline \multirow{14}{*}{$T_{C}$} & \multirow{5}{*}{$1 / 3$} & 0.0 & - & - & - & - & - & $\cdot$ & 1.2281 & - & - \\
\hline & & 0.2 & - & - & $\therefore$ & - & - & - & 1.1170 & - & $\cdot$ \\
\hline & & 0.4 & - & - & - & - & - & $\cdot$ & - & - & . \\
\hline & & 0.6 & - & - &. & - & - & $\therefore$ & - & - & - \\
\hline & & $\begin{array}{l}0.8 \\
\end{array}$ & - & - & - & - & - & - & - & - & - \\
\hline & \multirow{4}{*}{1} & 0.0 & - & - & $\therefore$ & - & - & - & 1.07 .35 & - & $\cdot$ \\
\hline & & 0.2 & - & - & - & - & $\therefore$ & - & - & - & - \\
\hline & & 0.4 & - & - & - & . & - & - & - & - & - \\
\hline & & 0.6 & - & - & - & - & - & $\therefore$ & $\therefore$ & - & - \\
\hline & \multirow{5}{*}{3} & $\frac{0.8}{0.0}$ & - & - & - & - & - & - & - & $\therefore$ & - \\
\hline & & 0.0 & - & - & - & - & $\dot{-}$ & $\dot{-}$ & $\therefore$ & - & - \\
\hline & & 0.4 & - & - & - & - & $\dot{.}$ & - & - & - & - \\
\hline & & 0.6 & - & - & - & - & - & - & - & - & - \\
\hline & & 0.8 & $\cdot$ & - & - & - & - & - & $\cdot$ &. & - \\
\hline \multirow{15}{*}{$T_{M}$} & \multirow{5}{*}{$1 / 3$} & 0.0 & 1.0867 & - & - & 1.6000 & - & . & 1.9010 & 1.0785 & 1.0794 \\
\hline & & 0.2 & 1.0730 & - & - & 1.4969 & - & - & 1.8074 & - & - \\
\hline & & 0.4 & - & - & - & 1.3713 & - & - & 1.6872 & . & - \\
\hline & & 0.6 & - & - & $\therefore$ & 1.2185 & - & - & 1.5244 & - & - \\
\hline & & 0.8 & - & - & - & 1.0726 & - & - & 1.2834 & - & - \\
\hline & \multirow{5}{*}{1} & 0.0 & - & - & - & 1.2155 & $\div$ & - & 1.5409 & - & 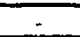 \\
\hline & & 0.2 & - & - & - & 1.1150 & - & - & 1.4413 & - &. \\
\hline & & 0.4 & - & - & - & - & - & - & 1.3172 & - & - \\
\hline & & 0.6 & - & - & - & - & - & - & 1.1576 & - & - \\
\hline & & $\begin{array}{l}0.8 \\
\end{array}$ & - & - & - & - & - &. & - & - & . \\
\hline & & 0.0 & - & - & - & - & - & - & 1.1716 & - & - \\
\hline & & 0.2 & $\therefore$ & - & $\therefore$ & - & - & - &. & - & - \\
\hline & 3 & 0.4 & - & - & - & - & $=$ & - & - & - & - \\
\hline & & 0.6 & - & - & - & - & - & - & - & - & - \\
\hline & & 0.8 & - & - & - & - & - & - & - & - & - \\
\hline & & 0.0 & - & - & - & 1.2672 & - & - & 1.5471 & - & - \\
\hline & & 0.2 & - & - & - & 1.1628 & - & - & 1.4462 & - & - \\
\hline & $1 / 3$ & 0.4 & - & - & - & - & - & - & 1.3196 & - & - \\
\hline & & 0.6 & - & - & - & - & - & - & 1.1549 & $\because$ & - \\
\hline & & 0.8 & - & - & - & - & $\div$ & - & - & - & - \\
\hline & & 0.0 & - & - & - & - & - & - & 1.2574 & - & - \\
\hline$T_{\text {Med }}$ & & 0.2 & - & - & - & - & - & - & 1.1539 & . & - \\
\hline${ }^{1}$ Med & 1 & 0.4 & - & - & - & . & - & - & - & - & - \\
\hline & & 0.6 & - & - & $=$ & - & - & - & $\therefore$ & - & . \\
\hline & & 0.8 & - & - & - & - & - & - & - & - & - \\
\hline & & 0.0 & - & - & - & $=$ & - & $\therefore$ & - & - & - \\
\hline & 3 & 0.2 & - & - & - & - & - & - & - & - & 二 \\
\hline & & $\begin{array}{l}0.4 \\
0.6\end{array}$ & - & $=$ & - & $\cdot$ & - & - & $\cdot$ & - & $\therefore$ \\
\hline & & $\begin{array}{l}0.0 \\
0.8\end{array}$ & - & $\because$ & $\therefore$ & - & - & $\dot{.}$ & $\frac{-}{-}$ & $\div$ & - \\
\hline & & 0.0 & - & - & - & - & - & - & 1.1227 & - & $\cdot$ \\
\hline & & 0.2 & - & - & - & - & - & - & $\cdot$ & - & - \\
\hline & $1 / 3$ & 0.4 & - & - & $\therefore$ & - & - & - & - & - & - \\
\hline & & 0.6 & - & - & - & - & - & - & - & - & - \\
\hline & & 0.8 & - & - & - & - & - & - & $\cdot$ & $\cdot$ & $\cdot$ \\
\hline & & 0.0 & - & - & $=$ & - & $\cdot$ & - & 1.2250 & - & - \\
\hline & & 0.2 & $\therefore$ & - & . & - & - & - & 1.10 .56 & - & - \\
\hline$T_{U}$ & 1 & 0.4 & - & $=$ & $\therefore$ & - & - & - & - & - & - \\
\hline & & 0.6 & - & - & - & - & - & - & - & - & - \\
\hline & & 0.8 & - & - & - & - & - & $=$ & - & $\cdot$ & - \\
\hline & & 0.0 & - & - & - & - & - & - & 1.1227 & - & - \\
\hline & & 0.2 & - & - & $\therefore$ & - & $\therefore$ & - & - & - & - \\
\hline & 3 & 0.4 & - & - & - & - & - & - & - & - & - \\
\hline & & 0.6 & - & - & $\therefore$ & - & - & - & - & - & - \\
\hline & & 0.8 & - & - & - & - & - & - & - & - & - \\
\hline
\end{tabular}


Suppressed average processing time results for the DPLFM with the task similarity factor $-C=250, N=2$

\begin{tabular}{|c|c|c|c|c|c|c|c|c|c|c|c|}
\hline \multirow{2}{*}{$\begin{array}{l}\text { Task- } \\
\text { Type }\end{array}$} & \multirow[b]{2}{*}{$R$} & \multirow[b]{2}{*}{$\begin{array}{l}\text { Similarity } \\
\text { Factor } \\
(S) \\
\end{array}$} & \multicolumn{3}{|c|}{$\begin{array}{l}\text { Low forgetting } \\
\text { rate of } 95 \%\end{array}$} & \multicolumn{3}{|c|}{$\begin{array}{l}\text { Medium forgetting } \\
\text { rate of } 90 \%\end{array}$} & \multicolumn{3}{|c|}{$\begin{array}{l}\text { High forgetting } \\
\text { rate of } 85 \%\end{array}$} \\
\hline & & & $\begin{array}{c}F A P \\
0\end{array}$ & $\begin{array}{c}F A P \\
1\end{array}$ & $\begin{array}{c}F A P \\
2\end{array}$ & $\begin{array}{c}F A P \\
0\end{array}$ & $\begin{array}{c}F A P \\
1\end{array}$ & $\begin{array}{c}F A P \\
2\end{array}$ & $\begin{array}{c}F A P \\
0\end{array}$ & $\begin{array}{c}F A P \\
1\end{array}$ & $\begin{array}{c}F A P \\
2\end{array}$ \\
\hline \multirow{14}{*}{$T_{C}$} & \multirow{5}{*}{$1 / 3$} & 0.0 & - & - & - & - & - & - & - & - & - \\
\hline & & 0.2 & - & - & - & - & $\therefore$ & - & - & - & - \\
\hline & & 0.4 & - & $\therefore$ & - & $\therefore$ & - & - & - & - & - \\
\hline & & 0.6 & $\cdot$ & - & $\therefore$ & - & - & - & - & - & - \\
\hline & & 0.8 & - & - & - & - & - & - & - & - & - \\
\hline & \multirow{5}{*}{1} & 0.0 & - & $\therefore$ & $\therefore$ & $\therefore$ & - & - & - & - & - \\
\hline & & 0.2 & $\cdot$ & $\therefore$ & - & - & - & - & - & - & - \\
\hline & & 0.4 & - & - & $=$ & - & - & - & - & - & - \\
\hline & & 0.6 & $\cdot$ &. & $\cdot$ & 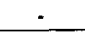 & - & - & - & - & - \\
\hline & & 0.8 & - & $\therefore$ & - & - & - & - & - & - & - \\
\hline & \multirow{5}{*}{3} & 0.0 & - & - & $=$ & $\therefore$ & - & - & - & - & - \\
\hline & & 0.2 & - & - & - & - & - & - & - & - & - \\
\hline & & 0.4 & $\therefore$ & - & $\therefore$ & - & - & - & - & - & - \\
\hline & & 0.6 & - & $\therefore$ & - & - & - & $\therefore$ & - & - & - \\
\hline \multirow{15}{*}{$T_{M}$} & & 0.8 & - & - & - & - & - & - & - & $=$ & - \\
\hline & \multirow{4}{*}{$1 / 3$} & 0.0 & - & $\therefore$ & $\therefore$ & 1.0760 & $\therefore$ & - & 1.1506 & $=$ & 1.0826 \\
\hline & & 0.2 & - & - & $\therefore$ & - & - & - & 1.1121 & - & 1.0746 \\
\hline & & 0.4 & - & - & $=$ & - & - & - & 1.0872 & - & - \\
\hline & & $\begin{array}{l}0.6 \\
0.8\end{array}$ & $\therefore$ & - & $\therefore$ & - & - & - & - & - & - \\
\hline & \multirow{5}{*}{1} & 0.8 & $\therefore$ & - & 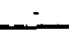 & - & - & - & $=$ & - & - \\
\hline & & 0.0 & - & - & $=$ & - & - & - & - & - & - \\
\hline & & & - & - & - & - & - & - & - & - & - \\
\hline & & 0.4 & - & - & $=$ & - & - & - & - & - & - \\
\hline & & 0.8 & - & - & - & - & - & - & - & - & - \\
\hline & & 0.0 & - & - & - & $=$ & - & - & - & - & - \\
\hline & & 0.2 & - & - & - & - & - & - & - & - & - \\
\hline & 3 & 0.4 & - & - & $\therefore$ & - & - & - & - & - & - \\
\hline & & 0.6 & $\because$ & - & - & - & - & - & - & - & - \\
\hline & & 0.8 & - & - & - & - & - & 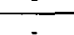 & - & - & - \\
\hline & & 0.0 & - & - & - & - & - & - & - & - & $\frac{-}{-}$ \\
\hline & & 0.2 & - & - & - & - & - & - & - & - & - \\
\hline & $1 / 3$ & 0.4 & - & $\therefore$ & $\therefore$ & - & - & $\therefore$ & - & - & - \\
\hline & & 0.6 & $\therefore$ & $\therefore$ & $\therefore$ & - & - & $\cdot$ & - & - & - \\
\hline & & 0.8 & - & - & - & - & - & - & - & - & - \\
\hline & & 0.0 & - & - & - & - & - & - & - & - & - \\
\hline & & 0.2 & - & - & - & - & - & - & - & - & - \\
\hline$I_{\text {Med }}$ & 1 & 0.4 & - & - & - & - & - & - & - & - & - \\
\hline & & 0.6 & - & - & $=$ & - & - & - & - & - & - \\
\hline & & 0.8 & - & - & - & - & - & - & - & - & - \\
\hline & & 0.0 & - & - & - & $=$ & - & - & - & - & - \\
\hline & & 0.2 & - & - & - & - & - & - & - & - & - \\
\hline & 3 & 0.4 & - & $\therefore$ & $\therefore$ & - & - & - & - & - & - \\
\hline & & 0.6 & - & $\therefore$ & $=$ & - & $=$ & - & - & - & - \\
\hline & & 0.8 & - & - & $\therefore$ & - & - & - & - & - & - \\
\hline & & 0.0 & - & - & - & - & - & - & - & - & - \\
\hline & & 0.2 & - & - & - & - & - & - & - & - & - \\
\hline & $1 / 3$ & 0.4 & - & $\therefore$ & - & - & $=$ & - & - & - & - \\
\hline & & 0.6 & - & - & - & - & - & - & - & - & - \\
\hline & & 0.8 & - & - & - & $\therefore$ & - & $=$ & - & - & - \\
\hline & & 0.0 & - & - & $=$ & - & - & - & - & - & - \\
\hline & 1 & 0.2 & $\therefore$ & - & - & - & $\therefore$ & $\therefore$ & - & - & - \\
\hline${ }^{1} U$ & 1 & 0.4 & - & - & - & - & - & - & - & - & - \\
\hline & & 0.6 & - & - & $=$ & - & - & - & - & - & - \\
\hline & & 0.8 & - & - & - & - & - & - & - & + & - \\
\hline & & 0.0 & - & $\therefore$ & $=$ & - & - & $\therefore$ & - & $=$ & - \\
\hline & & 0.2 & - & - & - & - & - & - & - & - & - \\
\hline & 3 & 0.4 & - & - & - & - & - & - & - & - & - \\
\hline & & 0.6 & - & - & - & - & - & 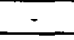 & - & $\therefore$ & - \\
\hline & & 0.8 & - & - & - & - & - & - & - & - & - \\
\hline
\end{tabular}




\subsubsection{Three tasks learned}

Suppressed average processing time results for the DPLFM with the task similarity factor $-C=10, N=3$

\begin{tabular}{|c|c|c|c|c|c|c|c|c|c|c|c|}
\hline \multirow[b]{2}{*}{$\begin{array}{l}\text { Task- } \\
\text { Type }\end{array}$} & \multirow[b]{2}{*}{$R$} & \multirow[b]{2}{*}{$\begin{array}{l}\text { Similarity } \\
\text { Factor }(S)\end{array}$} & \multicolumn{3}{|c|}{$\begin{array}{l}\text { Low forgetting } \\
\text { rate of } 95 \%\end{array}$} & \multicolumn{3}{|c|}{$\begin{array}{l}\text { Medium forgetting } \\
\text { rate of } 90 \%\end{array}$} & \multicolumn{3}{|c|}{$\begin{array}{l}\text { High forgetting } \\
\text { rate of } 85 \%\end{array}$} \\
\hline & & & $F A P-0$ & $F A P-1$ & $F A P-2$ & $F A P-0$ & $F A P-1$ & $F A P-2$ & $F A P-0$ & $F A P-1$ & $F A P-2$ \\
\hline \multirow{3}{*}{$T_{C}$} & $1 / 3$ & $\begin{array}{l}S_{0} \\
S_{1} \\
S_{2} \\
S_{3} \\
\end{array}$ & $\begin{array}{c}1.1263 \\
- \\
- \\
- \\
\end{array}$ & $\begin{array}{l}- \\
- \\
- \\
-\end{array}$ & $\begin{array}{l}- \\
- \\
- \\
- \\
\end{array}$ & $\begin{array}{c}1.7420 \\
1.6165 \\
1.4097 \\
-\quad \\
\end{array}$ & $\begin{array}{l}- \\
- \\
- \\
-\end{array}$ & $\begin{array}{l}- \\
- \\
- \\
\end{array}$ & $\begin{array}{l}2.0664 \\
1.9494 \\
1.7521 \\
1.2537\end{array}$ & $\begin{array}{c}1.2708 \\
1.1365 \\
- \\
-\end{array}$ & $\begin{array}{c}1.1274 \\
- \\
- \\
-\end{array}$ \\
\hline & 1 & $\begin{array}{l}\mathbf{S}_{\mathbf{0}} \\
\mathbf{S}_{1} \\
\mathbf{S}_{2} \\
\mathbf{S}_{3} \\
\end{array}$ & $\begin{array}{l}- \\
- \\
- \\
-\end{array}$ & $\begin{array}{l}- \\
- \\
- \\
-\end{array}$ & $\begin{array}{l}- \\
- \\
- \\
-\end{array}$ & $\begin{array}{c}1.4647 \\
1.3355 \\
1.1110 \\
. \\
\end{array}$ & $\begin{array}{l}- \\
- \\
- \\
-\end{array}$ & $\begin{array}{l}- \\
- \\
-\end{array}$ & $\begin{array}{c}1.8559 \\
1.7331 \\
1.5284 \\
-\end{array}$ & $\begin{array}{c}1.1903 \\
- \\
- \\
-\end{array}$ & $\begin{array}{l}- \\
- \\
-\end{array}$ \\
\hline & 3 & $\begin{array}{l}S_{0} \\
S_{1} \\
S_{2} \\
S_{3} \\
\end{array}$ & $\begin{array}{l}- \\
- \\
- \\
- \\
\end{array}$ & $\begin{array}{l}- \\
- \\
- \\
\end{array}$ & $\begin{array}{l}- \\
- \\
- \\
-\end{array}$ & $\begin{array}{c}1.0833 \\
- \\
- \\
- \\
\end{array}$ & $\begin{array}{l}- \\
- \\
- \\
- \\
\end{array}$ & $\begin{array}{l}- \\
- \\
- \\
-\end{array}$ & $\begin{array}{c}1.4711 \\
1.3361 \\
1.0946 \\
- \\
\end{array}$ & $\begin{array}{l}- \\
- \\
- \\
-\end{array}$ & $\begin{array}{l}- \\
\therefore \\
- \\
\end{array}$ \\
\hline \multirow{3}{*}{$T_{M}$} & $1 / 3$ & $\begin{array}{l}S_{0} \\
S_{1} \\
S_{2} \\
S_{3} \\
\end{array}$ & $\begin{array}{l}1.7488 \\
1.6160 \\
1.4007 \\
1.0733 \\
\end{array}$ & $\begin{array}{l}- \\
- \\
- \\
-\end{array}$ & $\begin{array}{l}- \\
- \\
- \\
-\end{array}$ & $\begin{array}{l}2.3971 \\
2.2934 \\
2.1180 \\
1.6663 \\
\end{array}$ & $\begin{array}{c}1.1351 \\
- \\
- \\
- \\
\end{array}$ & $\begin{array}{c}1.1079 \\
- \\
- \\
- \\
\end{array}$ & $\begin{array}{l}2.6494 \\
2.5583 \\
2.3982 \\
1.9671 \\
\end{array}$ & $\begin{array}{c}1.4259 \\
1.3008 \\
1.1180 \\
- \\
\end{array}$ & $\begin{array}{c}1.3012 \\
1.2062 \\
1.0936 \\
- \\
\end{array}$ \\
\hline & 1 & $\begin{array}{l}S_{0} \\
S_{1} \\
S_{2} \\
S_{3} \\
\end{array}$ & $\begin{array}{c}1.3887 \\
1.2530 \\
- \\
- \\
\end{array}$ & $\begin{array}{l}- \\
- \\
- \\
-\end{array}$ & $\begin{array}{l}- \\
- \\
- \\
-\end{array}$ & $\begin{array}{l}2.0008 \\
1.8835 \\
1.6888 \\
1.2186 \\
\end{array}$ & $\begin{array}{l}- \\
- \\
- \\
- \\
\end{array}$ & $\begin{array}{l}- \\
- \\
- \\
- \\
\end{array}$ & $\begin{array}{l}2.3408 \\
2.2349 \\
2.0543 \\
1.5878 \\
\end{array}$ & $\begin{array}{c}1.3416 \\
1.2105 \\
\cdot \\
- \\
\end{array}$ & $\begin{array}{c}1.2275 \\
1.1083 \\
- \\
- \\
\end{array}$ \\
\hline & 3 & $\begin{array}{l}S_{0} \\
S_{1} \\
S_{2} \\
S_{3}\end{array}$ & $\begin{array}{l}- \\
- \\
- \\
- \\
\end{array}$ & $\begin{array}{l}- \\
- \\
- \\
-\end{array}$ & $\begin{array}{l}- \\
- \\
- \\
-\end{array}$ & $\begin{array}{c}1.6476 \\
1.5207 \\
1.3132 \\
- \\
\end{array}$ & $\begin{array}{l}- \\
- \\
- \\
-\end{array}$ & $\begin{array}{l}- \\
- \\
- \\
-\end{array}$ & $\begin{array}{l}1.9818 \\
1.8627 \\
1.6637 \\
1.1724 \\
\end{array}$ & $\begin{array}{c}1.2181 \\
1.0833 \\
- \\
- \\
\end{array}$ & $\begin{array}{c}1.0805 \\
- \\
- \\
- \\
\end{array}$ \\
\hline \multirow{3}{*}{$T_{M e d}$} & $1 / 3$ & $\begin{array}{l}S_{0} \\
S_{1} \\
S_{2} \\
S_{3} \\
\end{array}$ & $\begin{array}{c}1.4156 \\
1.2772 \\
- \\
- \\
\end{array}$ & $\begin{array}{l}- \\
- \\
- \\
-\end{array}$ & $\begin{array}{l}- \\
- \\
- \\
-\end{array}$ & $\begin{array}{l}2.0616 \\
1.9451 \\
1.7505 \\
1.2689 \\
\end{array}$ & $\begin{array}{c}1.0919 \\
- \\
- \\
- \\
\end{array}$ & $\begin{array}{l}- \\
- \\
- \\
-\end{array}$ & $\begin{array}{l}2.3549 \\
2.2491 \\
2.0676 \\
1.5943 \\
\end{array}$ & $\begin{array}{c}1.3814 \\
1.2475 \\
- \\
- \\
\end{array}$ & $\begin{array}{c}1.2599 \\
1.1343 \\
- \\
- \\
\end{array}$ \\
\hline & 1 & $\begin{array}{l}S_{0} \\
S_{1} \\
S_{2} \\
S_{3}\end{array}$ & $\begin{array}{c}1.0811 \\
- \\
- \\
-\end{array}$ & $\begin{array}{l}- \\
- \\
- \\
\end{array}$ & $\begin{array}{l}- \\
- \\
- \\
-\end{array}$ & $\begin{array}{c}1.7204 \\
1.5957 \\
1.3911 \\
- \\
\end{array}$ & $\begin{array}{l}- \\
- \\
- \\
- \\
\end{array}$ & $\begin{array}{l}- \\
- \\
- \\
-\end{array}$ & $\begin{array}{l}2.0906 \\
1.9751 \\
1.7803 \\
1.2895 \\
\end{array}$ & $\begin{array}{c}.2697 \\
1.1 .379 \\
- \\
-\end{array}$ & $\begin{array}{c}1.1301 \\
- \\
- \\
-\end{array}$ \\
\hline & 3 & $\begin{array}{l}S_{0} \\
S_{1} \\
S_{2} \\
S_{3} \\
\end{array}$ & $\begin{array}{l}- \\
- \\
- \\
- \\
\end{array}$ & $\begin{array}{l}- \\
- \\
- \\
\end{array}$ & $\begin{array}{l}- \\
- \\
- \\
-\end{array}$ & $\begin{array}{c}1.3773 \\
1.2432 \\
- \\
- \\
\end{array}$ & $\begin{array}{l}- \\
- \\
- \\
-\end{array}$ & $\begin{array}{l}- \\
- \\
- \\
-\end{array}$ & $\begin{array}{c}1.7287 \\
1.6030 \\
1.3956 \\
- \\
\end{array}$ & $\begin{array}{c}1.1005 \\
- \\
- \\
. \\
\end{array}$ & $\begin{array}{l}- \\
- \\
-\end{array}$ \\
\hline \multirow{3}{*}{$T_{U}$} & $1 / 3$ & $\begin{array}{l}S_{0} \\
S_{1} \\
S_{2} \\
S_{3} \\
\end{array}$ & $\begin{array}{l} \\
- \\
- \\
- \\
\end{array}$ & $\begin{array}{l}- \\
- \\
- \\
-\end{array}$ & $\begin{array}{l}- \\
- \\
- \\
-\end{array}$ & $\begin{array}{c}1.6480 \\
1.5175 \\
1.3025 \\
- \\
\end{array}$ & $\begin{array}{l}- \\
- \\
- \\
-\end{array}$ & $\begin{array}{l}- \\
- \\
- \\
-\end{array}$ & $\begin{array}{l}1.9866 \\
1.8654 \\
1.6615 \\
1.1477\end{array}$ & $\begin{array}{c}\mathbf{1 . 2 2 7 1} \\
1.0824 \\
- \\
- \\
\end{array}$ & $\begin{array}{l}- \\
- \\
- \\
-\end{array}$ \\
\hline & 1 & $\begin{array}{l}S_{0} \\
S_{1} \\
S_{2} \\
S_{3} \\
\end{array}$ & $\begin{array}{c}1.0850 \\
- \\
- \\
- \\
\end{array}$ & $\begin{array}{l}- \\
- \\
- \\
-\end{array}$ & $\begin{array}{l}- \\
- \\
- \\
-\end{array}$ & $\begin{array}{c}1.7472 \\
1.6194 \\
1.4075 \\
- \\
\end{array}$ & $\begin{array}{l}- \\
- \\
- \\
-\end{array}$ & $\begin{array}{l}- \\
- \\
- \\
-\end{array}$ & $\begin{array}{l}2.1198 \\
2.0034 \\
1.8045 \\
1.2905 \\
\end{array}$ & $\begin{array}{c}1.2937 \\
1.1559 \\
- \\
- \\
\end{array}$ & $\begin{array}{c}1.1291 \\
- \\
- \\
- \\
\end{array}$ \\
\hline & 3 & $\begin{array}{l}S_{0} \\
S_{1} \\
S_{2} \\
S_{3}\end{array}$ & $\begin{array}{l}- \\
- \\
- \\
-\end{array}$ & $\begin{array}{l}- \\
- \\
-\end{array}$ & $\begin{array}{l}- \\
- \\
-\end{array}$ & $\begin{array}{c}1.6480 \\
1.5175 \\
1.3025 \\
-\quad\end{array}$ & $\begin{array}{l}- \\
- \\
- \\
-\end{array}$ & $\begin{array}{l} \\
- \\
- \\
\end{array}$ & $\begin{array}{l}1.9866 \\
1.8654 \\
1.6615 \\
1.1477\end{array}$ & $\begin{array}{c}1.2271 \\
1.0824 \\
- \\
-\end{array}$ & $\begin{array}{l}\cdot \\
- \\
- \\
-\end{array}$ \\
\hline
\end{tabular}


Average processing time results for the DPLFM with the task similarity factor $-C=25$, $N=3$

\begin{tabular}{|c|c|c|c|c|c|c|c|c|c|c|c|}
\hline \multirow[b]{2}{*}{$\begin{array}{l}\text { Task- } \\
\text { Type }\end{array}$} & \multirow[b]{2}{*}{$R$} & \multirow[b]{2}{*}{$\begin{array}{l}\text { Similarity } \\
\text { Factor }(S)\end{array}$} & \multicolumn{3}{|c|}{$\begin{array}{l}\text { Low forgetting } \\
\text { rate of } 95 \%\end{array}$} & \multicolumn{3}{|c|}{$\begin{array}{l}\text { Medium forgetting } \\
\text { rate of } 90 \%\end{array}$} & \multicolumn{3}{|c|}{$\begin{array}{l}\text { High forgetting } \\
\text { rate of } 85 \%\end{array}$} \\
\hline & & & $\begin{array}{c}F A P \\
0\end{array}$ & $\begin{array}{c}F A P \\
1\end{array}$ & $\begin{array}{c}F A P \\
2 \\
\end{array}$ & $\begin{array}{c}F A P \\
0 \\
\end{array}$ & $\begin{array}{c}F A P \\
1 \\
\end{array}$ & $\begin{array}{c}F A P \\
2 \\
\end{array}$ & $\begin{array}{c}F A P \\
0 \\
\end{array}$ & $\begin{array}{c}F A P \\
1 \\
\end{array}$ & $\begin{array}{c}F A P \\
2 \\
\end{array}$ \\
\hline \multirow{3}{*}{$T_{C}$} & $1 / 3$ & $\begin{array}{l}\mathbf{S}_{\mathfrak{y}} \\
\mathbf{S}_{1} \\
\mathbf{S}_{2} \\
\mathbf{S}_{3}\end{array}$ & $\begin{array}{l}- \\
- \\
- \\
\end{array}$ & $\begin{array}{l}- \\
- \\
- \\
-\end{array}$ & $\begin{array}{l}- \\
- \\
- \\
-\end{array}$ & $\begin{array}{c}1.2385 \\
1.1208 \\
- \\
- \\
\end{array}$ & $\begin{array}{l}- \\
- \\
- \\
\end{array}$ & $\begin{array}{l}- \\
- \\
- \\
- \\
\end{array}$ & $\begin{array}{c}1.5078 \\
1.4096 \\
1.2391 \\
- \\
\end{array}$ & $\begin{array}{c}1.1533 \\
- \\
- \\
- \\
\end{array}$ & $\begin{array}{l}- \\
- \\
- \\
\end{array}$ \\
\hline & 1 & $\begin{array}{l}\mathbf{S}_{0} \\
\mathbf{S}_{1} \\
\mathbf{S}_{2} \\
\mathbf{S}_{3} \\
\end{array}$ & . & $\begin{array}{l}- \\
- \\
-\end{array}$ & $\begin{array}{l}- \\
- \\
- \\
.\end{array}$ & $\begin{array}{l}- \\
- \\
- \\
- \\
\end{array}$ & $\begin{array}{l}- \\
- \\
- \\
-\end{array}$ & $\begin{array}{l}- \\
- \\
- \\
-\end{array}$ & $\begin{array}{c}1.2545 \\
1.1466 \\
1.0739 \\
-\quad \\
\end{array}$ & $\begin{array}{c}1.0828 \\
- \\
- \\
- \\
\end{array}$ & $\begin{array}{l}- \\
- \\
- \\
-\end{array}$ \\
\hline & 3 & $\begin{array}{l}S_{0} \\
S_{1} \\
S_{2} \\
S_{3} \\
\end{array}$ & $\begin{array}{l}- \\
- \\
- \\
\end{array}$ & $\begin{array}{l}- \\
- \\
- \\
-\end{array}$ & $\begin{array}{l}- \\
- \\
- \\
-\end{array}$ & $\begin{array}{l} \\
- \\
- \\
\end{array}$ & $\begin{array}{l} \\
- \\
- \\
-\end{array}$ & $\begin{array}{l} \\
- \\
- \\
\end{array}$ & $\begin{array}{c}1.0870 \\
- \\
- \\
- \\
\end{array}$ & $\begin{array}{l}- \\
- \\
- \\
- \\
\end{array}$ & $\begin{array}{l}- \\
- \\
- \\
\end{array}$ \\
\hline \multirow{3}{*}{$T_{M}$} & $1 / 3$ & $\begin{array}{l}S_{0} \\
S_{1} \\
S_{2} \\
S_{3} \\
\end{array}$ & $\begin{array}{c}1.3159 \\
1.2082 \\
1.0922 \\
- \\
\end{array}$ & $\begin{array}{l}- \\
- \\
- \\
-\end{array}$ & - & $\begin{array}{l}1.8715 \\
1.7755 \\
1.6124 \\
1.2139 \\
\end{array}$ & $\begin{array}{c}1.1131 \\
- \\
- \\
- \\
\end{array}$ & $\begin{array}{c}1.0990 \\
- \\
- \\
- \\
\end{array}$ & $\begin{array}{l}2.1302 \\
2.0493 \\
1.9061 \\
1.5191 \\
\end{array}$ & $\begin{array}{c}1.3605 \\
1.2582 \\
1.1027 \\
- \\
\end{array}$ & $\begin{array}{c}1.2634 \\
1.1855 \\
1.0877 \\
- \\
\end{array}$ \\
\hline & 1 & $\begin{array}{l}S_{0} \\
S_{1} \\
S_{2} \\
S_{3} \\
\end{array}$ & $\begin{array}{l}- \\
- \\
- \\
\end{array}$ & $\begin{array}{l}- \\
- \\
- \\
.\end{array}$ & $\begin{array}{l}- \\
- \\
-\end{array}$ & $\begin{array}{c}1.5121 \\
1.4090 \\
1.2382 \\
. \\
\end{array}$ & $\begin{array}{l}- \\
- \\
- \\
- \\
\end{array}$ & $\begin{array}{l}- \\
- \\
- \\
-\end{array}$ & $\begin{array}{l}1.8015 \\
1.7103 \\
1.5538 \\
1.1560 \\
\end{array}$ & $\begin{array}{c}1.2623 \\
1.1565 \\
- \\
- \\
\end{array}$ & $\begin{array}{c}1.1718 \\
- \\
- \\
- \\
\end{array}$ \\
\hline & 3 & $\begin{array}{l}S_{0} \\
S_{1} \\
S_{2} \\
S_{3}\end{array}$ & $\begin{array}{l}- \\
- \\
- \\
\end{array}$ & $\begin{array}{l}- \\
- \\
- \\
-\end{array}$ & $\begin{array}{r}- \\
- \\
-\end{array}$ & $\begin{array}{c}1.1711 \\
- \\
- \\
- \\
\end{array}$ & $\begin{array}{l}- \\
- \\
-\end{array}$ & $\begin{array}{l}- \\
- \\
- \\
-\end{array}$ & $\begin{array}{c}1.4555 \\
1.3554 \\
1.1817 \\
-\quad \\
\end{array}$ & $\begin{array}{c}1.1062 \\
- \\
- \\
- \\
\end{array}$ & $\begin{array}{l}- \\
- \\
- \\
-\end{array}$ \\
\hline \multirow{3}{*}{$T_{\text {Med }}$} & $1 / 3$ & $\begin{array}{l}S_{0} \\
S_{1} \\
S_{2} \\
S_{3} \\
\end{array}$ & $\begin{array}{l}- \\
- \\
- \\
\end{array}$ & $\begin{array}{l}- \\
- \\
- \\
\end{array}$ & $\begin{array}{l}- \\
- \\
- \\
-\end{array}$ & $\begin{array}{c}1.5616 \\
1.4593 \\
1.2879 \\
- \\
\end{array}$ & $\begin{array}{l}- \\
- \\
-\end{array}$ & $\begin{array}{l}- \\
- \\
- \\
\end{array}$ & $\begin{array}{l}1.8090 \\
1.7180 \\
1.5606 \\
1.1541\end{array}$ & $\begin{array}{c}1.2912 \\
1.1836 \\
- \\
-\end{array}$ & $\begin{array}{c}1.1936 \\
1.0884 \\
- \\
-\end{array}$ \\
\hline & 1 & $\begin{array}{l}S_{0} \\
S_{1} \\
S_{2} \\
S_{3} \\
\end{array}$ & $\begin{array}{l}- \\
- \\
- \\
\end{array}$ & $\begin{array}{l}- \\
- \\
- \\
-\end{array}$ & $\begin{array}{l}- \\
- \\
- \\
-\end{array}$ & $\begin{array}{c}1.2253 \\
1.1081 \\
- \\
- \\
\end{array}$ & $\begin{array}{l}- \\
- \\
-\end{array}$ & $\begin{array}{l}- \\
- \\
- \\
-\end{array}$ & $\begin{array}{c}1.5335 \\
1.4371 \\
1.2712 \\
- \\
\end{array}$ & $\begin{array}{c}1.1612 \\
- \\
- \\
- \\
\end{array}$ & $\begin{array}{l} \\
- \\
- \\
- \\
\end{array}$ \\
\hline & 3 & $\begin{array}{l}S_{0} \\
S_{1} \\
S_{2} \\
S_{3} \\
\end{array}$ & $\begin{array}{l}- \\
- \\
- \\
\end{array}$ & $\begin{array}{l}- \\
- \\
-\end{array}$ & $\begin{array}{l}- \\
- \\
-\end{array}$ & $\begin{array}{l}- \\
- \\
- \\
-\end{array}$ & $\begin{array}{l}- \\
- \\
- \\
-\end{array}$ & $\begin{array}{l}- \\
- \\
-\end{array}$ & $\begin{array}{c}1.1561 \\
1.0996 \\
- \\
-\end{array}$ & $\begin{array}{l}- \\
- \\
-\end{array}$ & $\begin{array}{l}- \\
- \\
- \\
-\end{array}$ \\
\hline \multirow{3}{*}{$T_{U}$} & $1 / 3$ & $\begin{array}{l}S_{0} \\
S_{1} \\
S_{1} \\
S_{3} \\
\end{array}$ & $\begin{array}{l}- \\
- \\
- \\
-\end{array}$ & $\begin{array}{l}- \\
- \\
-\end{array}$ & $\begin{array}{l}- \\
- \\
- \\
\end{array}$ & $\begin{array}{c}1.1251 \\
- \\
- \\
- \\
\end{array}$ & - & $\begin{array}{l}- \\
- \\
- \\
-\end{array}$ & $\begin{array}{c}1.4220 \\
1.3178 \\
1.1311 \\
. \quad \\
\end{array}$ & $\begin{array}{c}1.0879 \\
- \\
- \\
- \\
\end{array}$ & $\begin{array}{l}- \\
- \\
- \\
-\end{array}$ \\
\hline & 1 & $\begin{array}{l}S_{1} \\
S_{1} \\
S_{2} \\
S_{3} \\
\end{array}$ & $\begin{array}{l}- \\
- \\
- \\
-\end{array}$ & $\begin{array}{l}- \\
- \\
- \\
-\end{array}$ & $\begin{array}{l}- \\
- \\
- \\
\end{array}$ & $\begin{array}{c}1.1932 \\
1.0789 \\
- \\
- \\
\end{array}$ & $\begin{array}{l}. \\
- \\
-\end{array}$ & $\begin{array}{l}- \\
- \\
- \\
-\end{array}$ & $\begin{array}{c}1.5065 \\
1.4074 \\
1.2327 \\
-\quad \\
\end{array}$ & $\begin{array}{c}1.1561 \\
- \\
- \\
- \\
\end{array}$ & $\begin{array}{l}- \\
- \\
- \\
-\end{array}$ \\
\hline & 3 & $\begin{array}{l}S_{0} \\
S_{1} \\
S_{2} \\
S_{3} \\
\end{array}$ & $\begin{array}{l}- \\
- \\
- \\
\end{array}$ & $\begin{array}{l}- \\
- \\
- \\
-\end{array}$ & $\begin{array}{l}- \\
- \\
-\end{array}$ & $\begin{array}{c}1.1251 \\
- \\
- \\
- \\
\end{array}$ & $\begin{array}{l}- \\
- \\
- \\
\end{array}$ & $\begin{array}{l}- \\
- \\
- \\
\end{array}$ & $\begin{array}{c}1.4220 \\
1.3178 \\
1.1311 \\
-\quad \\
\end{array}$ & $\begin{array}{c}1.0879 \\
- \\
- \\
-\end{array}$ & $\begin{array}{l}- \\
- \\
-\end{array}$ \\
\hline
\end{tabular}


Average processing time results for the DPLFM with the task similarity factor $-C=250$, $N=3$

\begin{tabular}{|c|c|c|c|c|c|c|c|c|c|c|c|}
\hline \multirow[b]{2}{*}{$\begin{array}{l}\text { Task- } \\
\text { Type }\end{array}$} & \multirow[b]{2}{*}{$R$} & \multirow[b]{2}{*}{$\begin{array}{l}\text { Similarity } \\
\text { Factor }(S)\end{array}$} & \multicolumn{3}{|c|}{$\begin{array}{l}\text { Low forgetting } \\
\text { rate of } 95 \%\end{array}$} & \multicolumn{3}{|c|}{$\begin{array}{l}\text { Medium forgetting } \\
\text { rate of } 90 \%\end{array}$} & \multicolumn{3}{|c|}{$\begin{array}{l}\text { High forgetting } \\
\text { rate of } 85 \%\end{array}$} \\
\hline & & & $\begin{array}{c}F A P \\
0 \\
\end{array}$ & $\begin{array}{c}F A P \\
1 \\
\end{array}$ & $\begin{array}{c}F A P \\
2 \\
\end{array}$ & $\begin{array}{c}F A P \\
0 \\
\end{array}$ & $\begin{array}{c}F A P \\
1 \\
\end{array}$ & $\begin{array}{c}F A P \\
2 \\
\end{array}$ & $\begin{array}{c}F A P \\
0\end{array}$ & $\begin{array}{c}F A P \\
1\end{array}$ & $\begin{array}{c}F A P \\
2\end{array}$ \\
\hline \multirow{3}{*}{$T_{C}$} & $1 / 3$ & $\begin{array}{l}S_{0} \\
S_{1} \\
S_{2} \\
S_{3} \\
\end{array}$ & $\begin{array}{l}- \\
- \\
- \\
\end{array}$ & $\begin{array}{l}- \\
- \\
- \\
- \\
\end{array}$ & $\begin{array}{l} \\
- \\
- \\
\end{array}$ & $\begin{array}{l}- \\
- \\
- \\
-\end{array}$ & $\begin{array}{l}- \\
- \\
- \\
\end{array}$ & $\begin{array}{l} \\
- \\
- \\
\cdot\end{array}$ & $\begin{array}{l}- \\
- \\
- \\
\end{array}$ & $\begin{array}{l} \\
- \\
- \\
-\end{array}$ & $\begin{array}{l}\cdot \\
- \\
\cdot \\
-\end{array}$ \\
\hline & 1 & $\begin{array}{l}\mathbf{S}_{\mathbf{0}} \\
\mathbf{S}_{1} \\
\mathbf{S}_{2} \\
\mathbf{S}_{3} \\
\end{array}$ & $\begin{array}{l}- \\
- \\
- \\
-\end{array}$ & $\begin{array}{l}- \\
- \\
- \\
-\end{array}$ & $\begin{array}{l}- \\
- \\
- \\
-\end{array}$ & $\begin{array}{l}- \\
- \\
- \\
\end{array}$ & $\begin{array}{l}- \\
- \\
- \\
-\end{array}$ & $\begin{array}{l}- \\
- \\
- \\
\end{array}$ & $\begin{array}{l}- \\
- \\
- \\
-\end{array}$ & $\begin{array}{l}- \\
- \\
- \\
\end{array}$ & . \\
\hline & 3 & $\begin{array}{l}S_{0} \\
S_{1} \\
S_{2} \\
S_{3} \\
\end{array}$ & $\begin{array}{l}- \\
- \\
-\end{array}$ & $\begin{array}{l}- \\
- \\
- \\
-\end{array}$ & $\begin{array}{l}- \\
- \\
- \\
-\end{array}$ & $\begin{array}{l}- \\
- \\
- \\
-\end{array}$ & $\begin{array}{l}- \\
- \\
- \\
-\end{array}$ & $\begin{array}{l}- \\
- \\
- \\
\end{array}$ & $\begin{array}{l} \\
- \\
-\end{array}$ & $\begin{array}{l}- \\
- \\
-\end{array}$ & $\begin{array}{l}\dot{ } \cdot \\
- \\
-\end{array}$ \\
\hline \multirow{3}{*}{$T_{M}$} & $1 / 3$ & $\begin{array}{l}\mathbf{S}_{\mathbf{0}} \\
\mathbf{S}_{1} \\
\mathbf{S}_{2} \\
\mathbf{S}_{3} \\
\end{array}$ & $\begin{array}{l}- \\
- \\
- \\
-\end{array}$ & $\begin{array}{l}- \\
- \\
- \\
-\end{array}$ & $\begin{array}{l}- \\
- \\
- \\
-\end{array}$ & $\begin{array}{c}1.1191 \\
1.0958 \\
1.0736 \\
- \\
\end{array}$ & $\begin{array}{l}- \\
- \\
- \\
-\end{array}$ & $\begin{array}{c}1.0798 \\
1.0729 \\
\cdot \\
.\end{array}$ & $\begin{array}{c}1.2789 \\
1.2293 \\
1.1434 \\
-\quad \\
\end{array}$ & $\begin{array}{c}1.1234 \\
1.0771 \\
\cdot \\
.\end{array}$ & $\begin{array}{c}1.1253 \\
1.1013 \\
1.0804 \\
-\quad \\
\end{array}$ \\
\hline & 1 & $\begin{array}{l}\mathbf{S}_{0} \\
\mathbf{S}_{1} \\
\mathbf{S}_{2} \\
\mathbf{S}_{3} \\
\end{array}$ & $\begin{array}{l}- \\
- \\
- \\
-\end{array}$ & $\begin{array}{l}- \\
- \\
- \\
-\end{array}$ & $\begin{array}{l}- \\
- \\
-\end{array}$ & $\begin{array}{l}- \\
- \\
- \\
-\end{array}$ & $\begin{array}{l}- \\
- \\
- \\
-\end{array}$ & $\begin{array}{l} \\
- \\
- \\
-\end{array}$ & $\begin{array}{c}1.0836 \\
\cdot \\
- \\
-\end{array}$ & $\begin{array}{l}- \\
- \\
- \\
-\end{array}$ & $\begin{array}{c}1.0702 \\
\cdot \\
\cdot \\
- \\
\end{array}$ \\
\hline & 3 & $\begin{array}{l}S_{0} \\
S_{1} \\
S_{2} \\
S_{3} \\
\end{array}$ & $\begin{array}{l}- \\
- \\
- \\
-\end{array}$ & $\begin{array}{l}- \\
- \\
- \\
-\end{array}$ & $\begin{array}{l}- \\
- \\
- \\
\end{array}$ & $\begin{array}{l}- \\
- \\
- \\
-\end{array}$ & $\begin{array}{l}- \\
- \\
- \\
-\end{array}$ & $\begin{array}{l}- \\
- \\
- \\
-\end{array}$ & $\begin{array}{l}\cdot \\
- \\
- \\
\end{array}$ & $\begin{array}{l}- \\
- \\
- \\
\end{array}$ & $\begin{array}{l}- \\
- \\
- \\
\end{array}$ \\
\hline \multirow{3}{*}{$T_{M e d}$} & $1 / 3$ & $\begin{array}{l}S_{0} \\
S_{1} \\
S_{2} \\
S_{3} \\
\end{array}$ & $\begin{array}{l}- \\
- \\
- \\
-\end{array}$ & $\begin{array}{l}- \\
- \\
- \\
-\end{array}$ & $\begin{array}{l}- \\
- \\
- \\
-\end{array}$ & $\begin{array}{l}- \\
- \\
- \\
\end{array}$ & $\begin{array}{l}- \\
- \\
- \\
-\end{array}$ & $\begin{array}{l}- \\
. \\
-\end{array}$ & $\begin{array}{c}1.0818 \\
- \\
- \\
- \\
\end{array}$ & $\begin{array}{c}1.0711 \\
- \\
- \\
. \\
\end{array}$ & $\begin{array}{c}1.0717 \\
- \\
\cdot \\
- \\
\end{array}$ \\
\hline & 1 & $\begin{array}{l}\mathbf{S}_{\mathbf{0}} \\
\mathbf{S}_{1} \\
\mathbf{S}_{2} \\
\mathbf{S}_{\mathbf{3}} \\
\end{array}$ & $\begin{array}{l}- \\
- \\
- \\
-\end{array}$ & $\begin{array}{l}- \\
- \\
- \\
-\end{array}$ & $\begin{array}{l}- \\
- \\
- \\
-\end{array}$ & $\begin{array}{l}. \\
- \\
-\end{array}$ & $\begin{array}{l}- \\
- \\
- \\
\end{array}$ & $\begin{array}{l}. \\
. \\
-\end{array}$ & $\begin{array}{l}- \\
- \\
- \\
\end{array}$ & $\begin{array}{l}- \\
- \\
- \\
\end{array}$ & $\begin{array}{l}. \\
- \\
- \\
\end{array}$ \\
\hline & 3 & $\begin{array}{l}S_{0} \\
S_{1} \\
S_{2} \\
S_{3} \\
\end{array}$ & $\begin{array}{l}- \\
- \\
-\end{array}$ & $\begin{array}{l}- \\
- \\
- \\
-\end{array}$ & $\begin{array}{l}- \\
- \\
- \\
-\end{array}$ & $\begin{array}{l}- \\
- \\
- \\
\end{array}$ & $\begin{array}{l}- \\
- \\
- \\
-\end{array}$ & $\begin{array}{l}. \\
- \\
\end{array}$ & $\begin{array}{l}- \\
- \\
- \\
\end{array}$ & $\begin{array}{l}\cdot \\
- \\
.\end{array}$ & $\begin{array}{l}\cdot \\
. \\
.\end{array}$ \\
\hline \multirow{3}{*}{$T_{U}$} & $1 / 3$ & $\begin{array}{l}S_{0} \\
S_{1} \\
S_{2} \\
S_{3} \\
\end{array}$ & $\begin{array}{l}- \\
- \\
-\end{array}$ & $\begin{array}{l}- \\
- \\
- \\
-\end{array}$ & $\begin{array}{l}- \\
- \\
- \\
\end{array}$ & $\begin{array}{l}- \\
- \\
- \\
\end{array}$ & $\begin{array}{l}- \\
- \\
- \\
\end{array}$ & $\begin{array}{l}- \\
- \\
-\end{array}$ & $\begin{array}{l}- \\
- \\
- \\
\end{array}$ & $\begin{array}{l}- \\
- \\
. \\
\end{array}$ & $\begin{array}{l}. \\
. \\
.\end{array}$ \\
\hline & 1 & $\begin{array}{l}S_{\mathbf{0}} \\
S_{1} \\
S_{2} \\
S_{3} \\
\end{array}$ & $\begin{array}{l} \\
- \\
- \\
-\end{array}$ & $\begin{array}{l}- \\
- \\
- \\
-\end{array}$ & $\begin{array}{l}- \\
- \\
- \\
\end{array}$ & $\begin{array}{l}- \\
- \\
- \\
\end{array}$ & $\begin{array}{l}- \\
- \\
- \\
\end{array}$ & $\begin{array}{l}- \\
- \\
- \\
\end{array}$ & $\begin{array}{l}- \\
- \\
- \\
\end{array}$ & $\begin{array}{l} \\
. \\
- \\
\end{array}$ & $\begin{array}{l}- \\
- \\
- \\
-\end{array}$ \\
\hline & 3 & $\begin{array}{l}\mathbf{S}_{\mathbf{0}} \\
\mathbf{S}_{1} \\
\mathbf{S}_{\mathbf{2}} \\
\mathbf{S}_{3} \\
\end{array}$ & $\begin{array}{l}- \\
- \\
- \\
-\end{array}$ & $\begin{array}{l}- \\
- \\
- \\
-\end{array}$ & $\begin{array}{l}- \\
- \\
- \\
-\end{array}$ & $\begin{array}{l}- \\
- \\
- \\
- \\
\end{array}$ & $\begin{array}{l}- \\
- \\
- \\
-\end{array}$ & $\begin{array}{l}- \\
. \\
- \\
\end{array}$ & $\begin{array}{l}- \\
- \\
- \\
-\end{array}$ & $\begin{array}{l}\cdot \\
\cdot \\
\cdot \\
\cdot \\
\end{array}$ & $\begin{array}{l}\cdot \\
- \\
- \\
\end{array}$ \\
\hline
\end{tabular}




\subsection{Model C results}

LFCM, $s=0$,

$N=2$

\begin{tabular}{|c|c|c|c|c|}
\hline Forgetting Rate & Learning Rate & $\begin{array}{l}\text { Initial time to standard time ratio } \\
(\rho)\end{array}$ & FAP & Batch Size \\
\hline \multirow{25}{*}{$95 \%$} & \multirow{5}{*}{$70 \%$} & 2 & 0 & 250 \\
\hline & & 3 & 5 & 10 \\
\hline & & 4 & 1 & 10 \\
\hline & & 5 & 1 & 10 \\
\hline & & 6 & 1 & 10 \\
\hline & \multirow{5}{*}{$75 \%$} & 2 & 0 & 250 \\
\hline & & 3 & 1 & 10 \\
\hline & & 4 & 1 & 10 \\
\hline & & 5 & 1 & 10 \\
\hline & & 6 & 1 & 10 \\
\hline & \multirow{5}{*}{$80 \%$} & 2 & 0 & 250 \\
\hline & & 3 & 1 & 10 \\
\hline & & 4 & 1 & 10 \\
\hline & & 5 & 1 & 10 \\
\hline & & 6 & 1 & 10 \\
\hline & \multirow{5}{*}{$85 \%$} & 2 & 0 & 250 \\
\hline & & 3 & 1 & 10 \\
\hline & & 4 & 1 & 10 \\
\hline & & 5 & 1 & 10 \\
\hline & & 6 & 1 & 229 \\
\hline & \multirow{5}{*}{$90 \%$} & 2 & 1 & 10 \\
\hline & & 3 & 1 & 231 \\
\hline & & 4 & 0.5 & 10 \\
\hline & & 5 & 0.5 & 10 \\
\hline & & 6 & 0.5 & 10 \\
\hline \multirow{25}{*}{$92.5 \%$} & \multirow{5}{*}{$70 \%$} & 2 & 0 & 250 \\
\hline & & 3 & 0 & 250 \\
\hline & & 4 & 1 & 10 \\
\hline & & 5 & 1 & 10 \\
\hline & & 6 & 1 & 10 \\
\hline & \multirow{5}{*}{$75 \%$} & 2 & 0 & 250 \\
\hline & & 3 & 0 & 250 \\
\hline & & 4 & 1 & 10 \\
\hline & & 5 & 1 & 10 \\
\hline & & 6 & 1 & 10 \\
\hline & \multirow{5}{*}{$80 \%$} & 2 & 0 & 250 \\
\hline & & 3 & 1 & 10 \\
\hline & & 4 & 1 & 10 \\
\hline & & 5 & 1 & 10 \\
\hline & & 6 & 1 & 10 \\
\hline & \multirow{5}{*}{$85 \%$} & 2 & 0 & 250 \\
\hline & & 3 & 1 & 10 \\
\hline & & 4 & 1 & 10 \\
\hline & & 5 & 1 & 10 \\
\hline & & 6 & 1 & 250 \\
\hline & \multirow{5}{*}{$90 \%$} & 2 & 1 & 10 \\
\hline & & 3 & 1 & 231 \\
\hline & & 4 & 0.5 & 10 \\
\hline & & 5 & 0.5 & 10 \\
\hline & & 6 & 0.5 & 10 \\
\hline
\end{tabular}




\begin{tabular}{|c|c|c|c|c|}
\hline \multicolumn{3}{|l|}{$\begin{array}{l}\mathrm{LFCM}, s=0 \\
N=2\end{array}$} & \multicolumn{2}{|c|}{ Optimal Policy } \\
\hline Forgetting Rate & Learning Rate & Initial time to standard time ratio $(\rho)$ & FAP & Batch Size \\
\hline \multirow{25}{*}{$90 \%$} & \multirow{5}{*}{$70 \%$} & 2 & 0 & 250 \\
\hline & & 3 & 0 & 250 \\
\hline & & 4 & 0 & 250 \\
\hline & & 5 & 1 & 10 \\
\hline & & 6 & 1 & 10 \\
\hline & \multirow{5}{*}{$75 \%$} & 2 & 0 & 250 \\
\hline & & 3 & 0 & 250 \\
\hline & & 4 & 1 & 10 \\
\hline & & 5 & 1 & 10 \\
\hline & & 6 & 1 & 10 \\
\hline & \multirow{5}{*}{$80 \%$} & 2 & 0 & 250 \\
\hline & & 3 & 0 & 250 \\
\hline & & 4 & 1 & 10 \\
\hline & & 5 & $\mathrm{I}$ & 10 \\
\hline & & 6 & 1 & 10 \\
\hline & \multirow{5}{*}{$85 \%$} & 2 & 0 & 250 \\
\hline & & 3 & 1 & 10 \\
\hline & & 4 & 1 & 10 \\
\hline & & 5 & 1 & 250 \\
\hline & & 6 & 1 & 230 \\
\hline & \multirow{5}{*}{$90 \%$} & 2 & 5 & 10 \\
\hline & & 3 & 1 & 250 \\
\hline & & 4 & 0.5 & 10 \\
\hline & & 5 & 0.5 & 10 \\
\hline & & 6 & 0.5 & 10 \\
\hline \multirow{25}{*}{$87.5 \%$} & \multirow{5}{*}{$70 \%$} & 2 & 0 & 250 \\
\hline & & 3 & 0 & 250 \\
\hline & & 4 & 0 & 250 \\
\hline & & 5 & 0 & 250 \\
\hline & & 6 & 1 & 10 \\
\hline & \multirow{5}{*}{$75 \%$} & 2 & 0 & 250 \\
\hline & & 3 & 0 & 250 \\
\hline & & 4 & 0 & 250 \\
\hline & & 5 & 1 & 10 \\
\hline & & 6 & 1 & 10 \\
\hline & \multirow{5}{*}{$80 \%$} & 2 & 0 & 250 \\
\hline & & 3 & 0 & 250 \\
\hline & & 4 & 1 & 10 \\
\hline & & 5 & 1 & 10 \\
\hline & & 6 & 1 & 10 \\
\hline & \multirow{5}{*}{$85 \%$} & 2 & 0 & 250 \\
\hline & & 3 & 1 & 10 \\
\hline & & 4 & 1 & 10 \\
\hline & & 5 & 1 & 250 \\
\hline & & 6 & 1 & 230 \\
\hline & \multirow{5}{*}{$90 \%$} & 2 & 5 & 10 \\
\hline & & 3 & 1 & 232 \\
\hline & & 4 & 0.5 & 10 \\
\hline & & 5 & 0.5 & 10 \\
\hline & & 6 & 0.5 & 10 \\
\hline
\end{tabular}




\begin{tabular}{|c|c|c|c|c|}
\hline \multicolumn{3}{|l|}{$\begin{array}{l}\text { LFCM, } \\
s=0, N=2\end{array}$} & \multicolumn{2}{|c|}{ Optimal Policy } \\
\hline Forgetting Rate & Learning Rate & Initial time to standard time ratio $(\rho)$ & FAP & Batch Size \\
\hline \multirow{25}{*}{$85 \%$} & \multirow{5}{*}{$70 \%$} & 2 & 0 & 250 \\
\hline & & 3 & 0 & 250 \\
\hline & & 4 & 0 & 250 \\
\hline & & 5 & 0 & 250 \\
\hline & & 6 & 0 & 250 \\
\hline & \multirow{5}{*}{$75 \%$} & 2 & 0 & 250 \\
\hline & & 3 & 0 & 250 \\
\hline & & 4 & 0 & 250 \\
\hline & & 5 & 1 & 10 \\
\hline & & 6 & 1 & 10 \\
\hline & \multirow{5}{*}{$80 \%$} & 2 & 0 & 250 \\
\hline & & 3 & 0 & 250 \\
\hline & & 4 & 1 & 10 \\
\hline & & 5 & 1 & 10 \\
\hline & & 6 & 1 & 10 \\
\hline & \multirow{5}{*}{$85 \%$} & 2 & 0 & 250 \\
\hline & & 3 & 5 & 10 \\
\hline & & 4 & 1 & 64 \\
\hline & & 5 & 1 & 250 \\
\hline & & 6 & 1 & 230 \\
\hline & \multirow{5}{*}{$90 \%$} & 2 & 5 & 10 \\
\hline & & 3 & 1 & 232 \\
\hline & & 4 & 0.5 & 10 \\
\hline & & 5 & 0.5 & 10 \\
\hline & & 6 & 0.5 & 10 \\
\hline
\end{tabular}


LFCM,

$s=0.2, N=$

2

\begin{tabular}{|c|c|c|c|c|}
\hline \multicolumn{3}{|c|}{2} & \\
\hline Forgetting Rate & Learning Rate & Initial time to standard time ratio $(\rho)$ & FAP & Batch Size \\
\hline \multirow{25}{*}{$95 \%$} & \multirow{5}{*}{$70 \%$} & 2 & 0 & 250 \\
\hline & & 3 & 5 & 10 \\
\hline & & 4 & 1 & 10 \\
\hline & & 5 & 1 & 10 \\
\hline & & 6 & 1 & 10 \\
\hline & \multirow{5}{*}{$75 \%$} & 2 & 5 & 10 \\
\hline & & 3 & 1 & 10 \\
\hline & & 4 & 1 & 10 \\
\hline & & 5 & 1 & 10 \\
\hline & & 6 & 1 & 10 \\
\hline & \multirow{5}{*}{$80 \%$} & 2 & 0 & 250 \\
\hline & & 3 & 1 & 10 \\
\hline & & 4 & 1 & 10 \\
\hline & & 5 & 1 & 10 \\
\hline & & 6 & 1 & 10 \\
\hline & \multirow{5}{*}{$85 \%$} & 2 & 1 & 10 \\
\hline & & 3 & 1 & 10 \\
\hline & & 4 & 1 & 10 \\
\hline & & 5 & 1 & 10 \\
\hline & & 6 & 1 & 229 \\
\hline & \multirow{5}{*}{$90 \%$} & 2 & 1 & 10 \\
\hline & & 3 & 1 & 202 \\
\hline & & 4 & 0.5 & 10 \\
\hline & & 5 & 0.5 & 10 \\
\hline & & 6 & 0.5 & 10 \\
\hline \multirow{25}{*}{$92.5 \%$} & \multirow{5}{*}{$70 \%$} & 2 & 0 & 250 \\
\hline & & 3 & 5 & 10 \\
\hline & & 4 & 1 & 10 \\
\hline & & 5 & 1 & 10 \\
\hline & & 6 & 1 & 10 \\
\hline & \multirow{5}{*}{$\mathbf{7 5 \%}$} & 2 & 0 & 250 \\
\hline & & 3 & 1 & 10 \\
\hline & & 4 & 1 & 10 \\
\hline & & 5 & 1 & 10 \\
\hline & & 6 & 1 & 10 \\
\hline & \multirow{5}{*}{$80 \%$} & 2 & 0 & 250 \\
\hline & & 3 & 1 & 10 \\
\hline & & 4 & 1 & 10 \\
\hline & & 5 & 1 & 10 \\
\hline & & 6 & 1 & 10 \\
\hline & \multirow{5}{*}{$85 \%$} & 2 & 0 & 250 \\
\hline & & 3 & 1 & 10 \\
\hline & & 4 & 1 & 10 \\
\hline & & 5 & 1 & 10 \\
\hline & & 6 & 1 & 229 \\
\hline & \multirow{5}{*}{$90 \%$} & 2 & 1 & 10 \\
\hline & & 3 & 1 & 231 \\
\hline & & 4 & 0.5 & 10 \\
\hline & & 5 & 0.5 & 10 \\
\hline & & 6 & 0.5 & 10 \\
\hline
\end{tabular}




\begin{tabular}{|c|c|c|c|c|}
\hline \multicolumn{3}{|l|}{$\begin{array}{l}\mathrm{LFCM}, \\
s=0.2, N= \\
2\end{array}$} & \multicolumn{2}{|c|}{ Optimal Policy } \\
\hline Forgetting Rate & Learning Rate & Initial time to standard time ratio $(\rho)$ & FAP & Batch Size \\
\hline \multirow{25}{*}{$90 \%$} & \multirow{5}{*}{$70 \%$} & 2 & 0 & 250 \\
\hline & & 3 & 0 & 250 \\
\hline & & 4 & 0 & 250 \\
\hline & & 5 & 1 & 10 \\
\hline & & 6 & 1 & 10 \\
\hline & \multirow{5}{*}{$75 \%$} & 2 & 0 & 250 \\
\hline & & 3 & 0 & 250 \\
\hline & & 4 & 1 & 10 \\
\hline & & 5 & 1 & 10 \\
\hline & & 6 & 1 & 10 \\
\hline & \multirow{5}{*}{$80 \%$} & 2 & 0 & 250 \\
\hline & & 3 & 1 & 10 \\
\hline & & 4 & 1 & 10 \\
\hline & & 5 & 1 & 10 \\
\hline & & 6 & 1 & 10 \\
\hline & \multirow{5}{*}{$85 \%$} & 2 & 0 & 250 \\
\hline & & 3 & 1 & 10 \\
\hline & & 4 & 1 & 10 \\
\hline & & 5 & 1 & 204 \\
\hline & & 6 & 1 & 230 \\
\hline & \multirow{5}{*}{$90 \%$} & 2 & 1 & 10 \\
\hline & & 3 & 1 & 250 \\
\hline & & 4 & 0.5 & 10 \\
\hline & & 5 & 0.5 & 10 \\
\hline & & 6 & 0.5 & 10 \\
\hline \multirow{25}{*}{$87.5 \%$} & \multirow{5}{*}{$70 \%$} & 2 & 0 & 250 \\
\hline & & 3 & 0 & 250 \\
\hline & & 4 & 0 & 250 \\
\hline & & 5 & 1 & 10 \\
\hline & & 6 & 1 & 10 \\
\hline & \multirow{5}{*}{$75 \%$} & 2 & 0 & 250 \\
\hline & & 3 & 0 & 250 \\
\hline & & 4 & 0 & 250 \\
\hline & & 5 & 1 & 10 \\
\hline & & 6 & 1 & 10 \\
\hline & \multirow{5}{*}{$80 \%$} & 2 & 0 & 250 \\
\hline & & 3 & 0 & 250 \\
\hline & & 4 & 1 & 10 \\
\hline & & 5 & 1 & 10 \\
\hline & & 6 & 1 & 10 \\
\hline & \multirow{5}{*}{$85 \%$} & 2 & 0 & 250 \\
\hline & & 3 & 1 & 10 \\
\hline & & 4 & 1 & 10 \\
\hline & & 5 & 1 & 250 \\
\hline & & 6 & 1 & 230 \\
\hline & \multirow{5}{*}{$90 \%$} & 2 & 5 & 10 \\
\hline & & 3 & 1 & 250 \\
\hline & & 4 & 0.5 & 10 \\
\hline & & 5 & 0.5 & 10 \\
\hline & & 6 & 0.5 & 10 \\
\hline
\end{tabular}




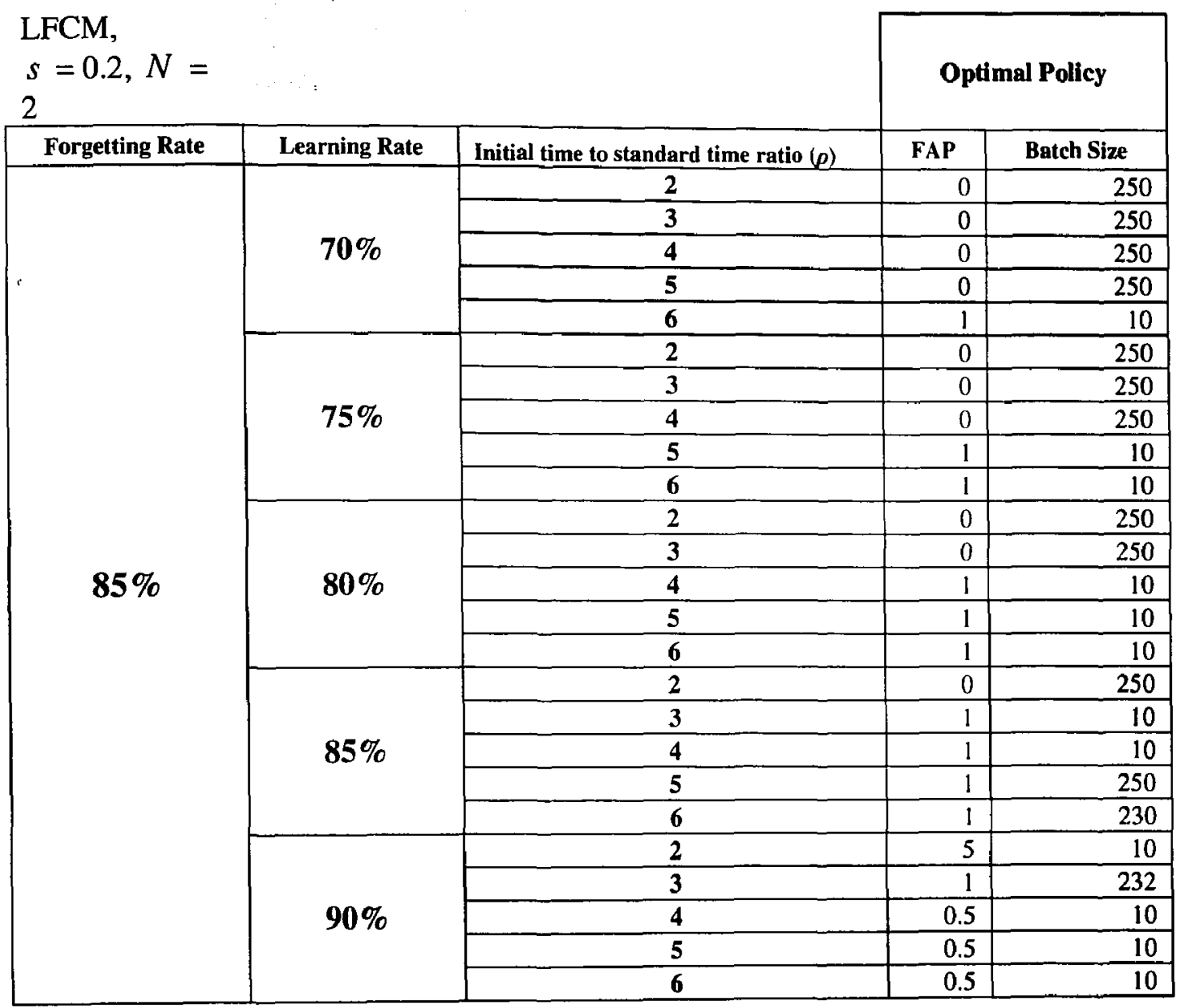




\begin{tabular}{|c|c|c|c|c|}
\hline \multicolumn{3}{|l|}{$\begin{array}{l}\text { LFCM, } \\
s=0.4, N= \\
2\end{array}$} & \multicolumn{2}{|c|}{ Optimal Policy } \\
\hline Forgetting Rate & Learning Rate & Initial time to standard time ratio $(\rho)$ & FAP & $\begin{array}{c}\text { Batch } \\
\text { Size }\end{array}$ \\
\hline \multirow{25}{*}{$95 \%$} & \multirow{5}{*}{$70 \%$} & 2 & 5 & 10 \\
\hline & & 3 & 5 & 10 \\
\hline & & 4 & 1 & 10 \\
\hline & & 5 & 1 & 10 \\
\hline & & 6 & 1 & 10 \\
\hline & \multirow{5}{*}{$75 \%$} & 2 & 5 & 17 \\
\hline & & 3 & 1 & 10 \\
\hline & & 4 & 1 & 10 \\
\hline & & 5 & 1 & 10 \\
\hline & & 6 & 1 & 10 \\
\hline & \multirow{5}{*}{$80 \%$} & 2 & 5 & 10 \\
\hline & & 3 & 1 & 10 \\
\hline & & 4 & 1 & 10 \\
\hline & & 5 & 1 & 10 \\
\hline & & 6 & 1 & 10 \\
\hline & \multirow{5}{*}{$85 \%$} & 2 & 1 & 10 \\
\hline & & 3 & 1 & 10 \\
\hline & & 4 & 1 & 10 \\
\hline & & 5 & 1 & 10 \\
\hline & & 6 & 1 & 229 \\
\hline & \multirow{5}{*}{$90 \%$} & 2 & 1 & 10 \\
\hline & & 3 & 1 & 231 \\
\hline & & 4 & 0.5 & 10 \\
\hline & & 5 & 0.5 & 10 \\
\hline & & 6 & 0.5 & 10 \\
\hline \multirow{25}{*}{$92.5 \%$} & \multirow{5}{*}{$70 \%$} & 2 & 0 & 250 \\
\hline & & 3 & 5 & 10 \\
\hline & & 4 & 1 & 10 \\
\hline & & 5 & 1 & 10 \\
\hline & & 6 & 1 & 10 \\
\hline & \multirow{5}{*}{$75 \%$} & 2 & 0 & 250 \\
\hline & & 3 & 1 & 10 \\
\hline & & 4 & 1 & 10 \\
\hline & & 5 & 1 & 10 \\
\hline & & 6 & 1 & 10 \\
\hline & \multirow{5}{*}{$80 \%$} & 2 & 0 & 250 \\
\hline & & 3 & 1 & 10 \\
\hline & & 4 & 1 & 10 \\
\hline & & 5 & 1 & 10 \\
\hline & & 6 & 1 & 10 \\
\hline & \multirow{5}{*}{$\mathbf{8 5 \%}$} & 2 & 0 & 250 \\
\hline & & 3 & 1 & 10 \\
\hline & & 4 & 1 & 10 \\
\hline & & 5 & 1 & 10 \\
\hline & & 6 & 1 & 229 \\
\hline & \multirow{5}{*}{$90 \%$} & 2 & 1 & 10 \\
\hline & & 3 & 1 & 231 \\
\hline & & 4 & 0.5 & 10 \\
\hline & & 5 & 0.5 & 10 \\
\hline & & 6 & 0.5 & 10 \\
\hline
\end{tabular}


LFCM,

$s=0.4, N=$

2

\begin{tabular}{|c|c|c|c|c|}
\hline Forgetting Rate & Learning Rate & Initial time to standard time ratio $(\rho)$ & FAP & Batch Size \\
\hline \multirow{25}{*}{$90 \%$} & \multirow{5}{*}{$70 \%$} & 2 & 0 & 250 \\
\hline & & 3 & 5 & 10 \\
\hline & & 4 & 1 & 10 \\
\hline & & 5 & 1 & 10 \\
\hline & & 6 & 1 & 10 \\
\hline & \multirow{5}{*}{$75 \%$} & 2 & 0 & 250 \\
\hline & & 3 & 0 & 250 \\
\hline & & 4 & 1 & 10 \\
\hline & & 5 & 1 & 10 \\
\hline & & 6 & 1 & 10 \\
\hline & \multirow{5}{*}{$80 \%$} & 2 & 0 & 250 \\
\hline & & 3 & 1 & 10 \\
\hline & & 4 & 1 & 10 \\
\hline & & 5 & 1 & 10 \\
\hline & & 6 & 1 & 10 \\
\hline & \multirow{5}{*}{$85 \%$} & 2 & 0 & 250 \\
\hline & & 3 & 1 & 10 \\
\hline & & 4 & 1 & 10 \\
\hline & & 5 & 1 & 10 \\
\hline & & 6 & 1 & 229 \\
\hline & \multirow{5}{*}{$90 \%$} & 2 & 1 & 10 \\
\hline & & 3 & 1 & 231 \\
\hline & & 4 & 0.5 & 10 \\
\hline & & 5 & 0.5 & 10 \\
\hline & & 6 & 0.5 & 10 \\
\hline \multirow{24}{*}{$87.5 \%$} & \multirow{5}{*}{$70 \%$} & 2 & 0 & 250 \\
\hline & & 3 & 0 & 250 \\
\hline & & 4 & 0 & 250 \\
\hline & & 5 & 1 & 10 \\
\hline & & 6 & 1 & 10 \\
\hline & \multirow{5}{*}{$75 \%$} & 2 & 0 & 250 \\
\hline & & 3 & 0 & 250 \\
\hline & & 4 & 1 & 10 \\
\hline & & 5 & 1 & 10 \\
\hline & & 6 & 1 & 10 \\
\hline & \multirow{5}{*}{$80 \%$} & 2 & 0 & 250 \\
\hline & & 3 & 1 & 10 \\
\hline & & 4 & 1 & 10 \\
\hline & & 5 & 1 & 10 \\
\hline & & 6 & 1 & 10 \\
\hline & \multirow{5}{*}{$85 \%$} & 2 & 0 & 250 \\
\hline & & 3 & 1 & 10 \\
\hline & & 4 & 1 & 10 \\
\hline & & 5 & 1 & 10 \\
\hline & & 6 & 1 & 230 \\
\hline & \multirow{5}{*}{$90 \%$} & 2 & 1 & 10 \\
\hline & & 3 & 1 & 250 \\
\hline & & 4 & 0.5 & 10 \\
\hline & & 3 & 0.5 & 10 \\
\hline & & 6 & 0.5 & 10 \\
\hline
\end{tabular}




\begin{tabular}{|c|c|c|c|c|}
\hline \multicolumn{3}{|l|}{$\begin{array}{l}\text { LFCM, } \\
s=0.4, N= \\
2\end{array}$} & \multicolumn{2}{|c|}{ Optimal Policy } \\
\hline Forgetting Rate & Learning Rate & Initial time to standard time ratio $(\rho)$ & FAP & Batch Size \\
\hline \multirow{25}{*}{$\mathbf{8 5 \%}$} & \multirow{5}{*}{$70 \%$} & 2 & 0 & 250 \\
\hline & & 3 & 0 & 250 \\
\hline & & 4 & 0 & 250 \\
\hline & & 5 & 1 & 10 \\
\hline & & 6 & 1 & 10 \\
\hline & \multirow{5}{*}{$75 \%$} & 2 & 0 & 250 \\
\hline & & 3 & 0 & 250 \\
\hline & & 4 & 1 & 10 \\
\hline & & 5 & 1 & 10 \\
\hline & & 6 & 1 & 10 \\
\hline & \multirow{5}{*}{$80 \%$} & 2 & 0 & 250 \\
\hline & & 3 & 0 & 250 \\
\hline & & 4 & 1 & 10 \\
\hline & & 5 & 1 & 10 \\
\hline & & 6 & 1 & 10 \\
\hline & \multirow{5}{*}{$85 \%$} & 2 & 0 & 250 \\
\hline & & 3 & 1 & 10 \\
\hline & & 4 & 1 & 10 \\
\hline & & 5 & 1 & 227 \\
\hline & & 6 & 1 & 230 \\
\hline & \multirow{5}{*}{$90 \%$} & 2 & 5 & 10 \\
\hline & & 3 & 1 & 250 \\
\hline & & 4 & 0.5 & 10 \\
\hline & & 5 & 0.5 & 10 \\
\hline & & 6 & 0.5 & 10 \\
\hline
\end{tabular}


LFCM,

$s=0.6, N=$

2

\begin{tabular}{|c|c|c|c|c|}
\hline Forgetting Rate & Learning Rate & Initial time to standard time ratio $(\rho)$ & FAP & Batch Size \\
\hline \multirow{25}{*}{$95 \%$} & \multirow{5}{*}{$70 \%$} & 2 & 5 & 10 \\
\hline & & 3 & 0 & 20 \\
\hline & & 4 & 1 & 10 \\
\hline & & 5 & 1 & 10 \\
\hline & & 6 & 1 & 10 \\
\hline & \multirow{5}{*}{$75 \%$} & 2 & 0 & 15 \\
\hline & & 3 & 1 & 10 \\
\hline & & 4 & 1 & 10 \\
\hline & & 5 & 1 & 10 \\
\hline & & 6 & 1 & 10 \\
\hline & \multirow{5}{*}{$80 \%$} & 2 & 5 & 10 \\
\hline & & 3 & 1 & 10 \\
\hline & & 4 & 1 & 10 \\
\hline & & 5 & 1 & 10 \\
\hline & & 6 & 1 & 10 \\
\hline & \multirow{5}{*}{$85 \%$} & 2 & 1 & 10 \\
\hline & & 3 & 1 & 10 \\
\hline & & 4 & 1 & 10 \\
\hline & & 5 & 1 & 10 \\
\hline & & 6 & 1 & 183 \\
\hline & \multirow{5}{*}{$90 \%$} & 2 & 1 & 10 \\
\hline & & 3 & 1 & 180 \\
\hline & & 4 & 0.5 & 10 \\
\hline & & 5 & 0.5 & 10 \\
\hline & & 6 & 0.5 & 10 \\
\hline \multirow{25}{*}{$92.5 \%$} & \multirow{5}{*}{$70 \%$} & 2 & 5 & 10 \\
\hline & & 3 & 5 & 10 \\
\hline & & 4 & 1 & 10 \\
\hline & & 5 & 1 & 10 \\
\hline & & 6 & 1 & 10 \\
\hline & \multirow{5}{*}{$75 \%$} & 2 & 5 & 17 \\
\hline & & 3 & 1 & 10 \\
\hline & & 4 & 1 & 10 \\
\hline & & 5 & 1 & 10 \\
\hline & & 6 & 1 & 10 \\
\hline & \multirow{5}{*}{$80 \%$} & 2 & 5 & 10 \\
\hline & & 3 & 1 & 10 \\
\hline & & 4 & 1 & 10 \\
\hline & & 5 & 1 & 10 \\
\hline & & 6 & 1 & 10 \\
\hline & \multirow{5}{*}{$85 \%$} & 2 & 1 & 10 \\
\hline & & 3 & 1 & 10 \\
\hline & & 4 & 1 & 10 \\
\hline & & 5 & 1 & 10 \\
\hline & & 6 & 1 & 229 \\
\hline & \multirow{5}{*}{$90 \%$} & 2 & 1 & 10 \\
\hline & & 3 & 1 & 231 \\
\hline & & 4 & 0.5 & 10 \\
\hline & & 5 & 0.5 & 10 \\
\hline & & 6 & 0.5 & 10 \\
\hline
\end{tabular}


LFCM,

$s=0.6, N=$

Optimal Policy

2

\begin{tabular}{|c|c|c|c|c|}
\hline Forgetting Rate & Learning Rate & Initial time to standard time ratio $(\rho)$ & FAP & Batch Size \\
\hline \multirow{24}{*}{$90 \%$} & \multirow{5}{*}{$70 \%$} & 2 & 0 & 250 \\
\hline & & 3 & 5 & 10 \\
\hline & & 4 & 1 & 10 \\
\hline & & 5 & 1 & 10 \\
\hline & & 6 & 1 & 10 \\
\hline & \multirow{5}{*}{$75 \%$} & 2 & 5 & 10 \\
\hline & & 3 & 1 & 10 \\
\hline & & 4 & 1 & 10 \\
\hline & & 5 & 1 & 10 \\
\hline & & $\frac{6}{2}$ & 1 & 10 \\
\hline & \multirow{4}{*}{$80 \%$} & $\frac{2}{3}$ & 0 & $\frac{250}{10}$ \\
\hline & & 4 & $\frac{1}{1}$ & $\frac{10}{10}$ \\
\hline & & 5 & 1 & 10 \\
\hline & & 6 & 1 & 10 \\
\hline & \multirow{5}{*}{$85 \%$} & 2 & 0 & 250 \\
\hline & & 3 & 1 & 10 \\
\hline & & 4 & 1 & 10 \\
\hline & & 5 & 1 & 10 \\
\hline & & 6 & 1 & 229 \\
\hline & \multirow{5}{*}{$90 \%$} & 2 & 1 & 10 \\
\hline & & 3 & 1 & 231 \\
\hline & & 4 & 0.5 & 10 \\
\hline & & 5 & 0.5 & 10 \\
\hline & & 6 & 0.5 & 10 \\
\hline \multirow{25}{*}{$87.5 \%$} & \multirow{5}{*}{$70 \%$} & 2 & 0 & 250 \\
\hline & & 3 & 5 & 10 \\
\hline & & 4 & 1 & 10 \\
\hline & & 5 & 1 & 10 \\
\hline & & 6 & 1 & 10 \\
\hline & \multirow{5}{*}{$75 \%$} & 2 & 0 & 250 \\
\hline & & 3 & 1 & 10 \\
\hline & & 4 & 1 & 10 \\
\hline & & 5 & 1 & 10 \\
\hline & & 6 & 1 & 10 \\
\hline & \multirow{5}{*}{$80 \%$} & 2 & 0 & 250 \\
\hline & & 3 & 1 & 10 \\
\hline & & 4 & 1 & 10 \\
\hline & & 5 & 1 & 10 \\
\hline & & 6 & 1 & 10 \\
\hline & \multirow{5}{*}{$85 \%$} & 2 & 0 & 250 \\
\hline & & 3 & 1 & 10 \\
\hline & & 4 & 1 & 10 \\
\hline & & 5 & 1 & 10 \\
\hline & & 6 & 1 & 229 \\
\hline & \multirow{5}{*}{$90 \%$} & 2 & 1 & 10 \\
\hline & & 3 & 1 & 231 \\
\hline & & 4 & 0.5 & 10 \\
\hline & & 5 & 0.5 & 10 \\
\hline & & 6 & 0.5 & 10 \\
\hline
\end{tabular}




\begin{tabular}{|c|c|c|c|c|}
\hline \multirow{2}{*}{$\begin{array}{l}\text { LFCM, } \\
s=0.6, N= \\
2 \\
\text { Forgetting Rate }\end{array}$} & & & \multicolumn{2}{|c|}{ Optimal Policy } \\
\hline & Learning Rate & Initial time to standard time ratio $(\rho)$ & FAP & Batch Size \\
\hline \multirow{25}{*}{$85 \%$} & \multirow{5}{*}{$70 \%$} & 2 & 0 & 250 \\
\hline & & 3 & 5 & 10 \\
\hline & & 4 & 1 & 10 \\
\hline & & 5 & 1 & 10 \\
\hline & & 6 & 1 & 10 \\
\hline & \multirow{5}{*}{$75 \%$} & 2 & 0 & 250 \\
\hline & & 3 & 0 & 250 \\
\hline & & 4 & 1 & 10 \\
\hline & & 5 & 1 & 10 \\
\hline & & 6 & 1 & 10 \\
\hline & \multirow{5}{*}{$80 \%$} & 2 & 0 & 250 \\
\hline & & 3 & 1 & 10 \\
\hline & & 4 & 1 & 10 \\
\hline & & 5 & 1 & 10 \\
\hline & & 6 & 1 & 10 \\
\hline & \multirow{5}{*}{$85 \%$} & 2 & 0 & 250 \\
\hline & & $\mathbf{3}$ & 1 & 10 \\
\hline & & 4 & 1 & 10 \\
\hline & & 5 & 1 & 10 \\
\hline & & 6 & 1 & 229 \\
\hline & \multirow{5}{*}{$90 \%$} & 2 & 1 & 10 \\
\hline & & 3 & 1 & 231 \\
\hline & & 4 & 0.5 & 10 \\
\hline & & 5 & 0.5 & 10 \\
\hline & & 6 & 0.5 & 10 \\
\hline
\end{tabular}




\begin{tabular}{|c|c|c|c|c|}
\hline \multicolumn{3}{|l|}{$\begin{array}{l}\text { LFCM, } \\
s=0.8, N= \\
2\end{array}$} & \multicolumn{2}{|c|}{ Optimal Policy } \\
\hline Forgetting Rate & Learning Rate & Initial time to standard time ratio $(\rho)$ & FAP & $\begin{array}{l}\text { Batch } \\
\text { Size }\end{array}$ \\
\hline \multirow{25}{*}{$95 \%$} & \multirow{5}{*}{$70 \%$} & 2 & 5 & 10 \\
\hline & & 3 & 0 & 10 \\
\hline & & $\overline{4}$ & 1 & 10 \\
\hline & & 5 & 1 & 10 \\
\hline & & 6 & 1 & 10 \\
\hline & \multirow{5}{*}{$75 \%$} & 2 & 0 & 10 \\
\hline & & 3 & 1 & 10 \\
\hline & & 4 & 1 & 10 \\
\hline & & 5 & 1 & 10 \\
\hline & & 6 & 1 & 10 \\
\hline & \multirow{5}{*}{$80 \%$} & 2 & 5 & 10 \\
\hline & & 3 & 1 & 10 \\
\hline & & 4 & 1 & 10 \\
\hline & & 5 & 1 & 10 \\
\hline & & 6 & 1 & 10 \\
\hline & \multirow{5}{*}{$85 \%$} & 2 & 1 & 10 \\
\hline & & 3 & 1 & 10 \\
\hline & & 4 & 1 & 10 \\
\hline & & 5 & 1 & 10 \\
\hline & & 6 & 1 & 91 \\
\hline & \multirow{5}{*}{$90 \%$} & 2 & 1 & 10 \\
\hline & & 3 & 1 & 10 \\
\hline & & 4 & 0.5 & 10 \\
\hline & & 5 & 0.5 & 10 \\
\hline & & 6 & 0.5 & 10 \\
\hline \multirow{25}{*}{$92.5 \%$} & \multirow{5}{*}{$70 \%$} & 2 & 5 & 10 \\
\hline & & 3 & 0 & 10 \\
\hline & & 4 & 1 & 10 \\
\hline & & 5 & 1 & 10 \\
\hline & & 6 & 1 & 10 \\
\hline & \multirow{5}{*}{$75 \%$} & 2 & 0 & 10 \\
\hline & & 3 & 1 & 10 \\
\hline & & 4 & 1 & 10 \\
\hline & & 5 & 1 & 10 \\
\hline & & 6 & 1 & 10 \\
\hline & \multirow{5}{*}{$80 \%$} & 2 & 5 & 10 \\
\hline & & 3 & 1 & 10 \\
\hline & & 4 & 1 & 10 \\
\hline & & 5 & 1 & 10 \\
\hline & & 6 & 1 & 10 \\
\hline & \multirow{5}{*}{$85 \%$} & 2 & 1 & 10 \\
\hline & & 3 & 1 & 10 \\
\hline & & 4 & 1 & 10 \\
\hline & & 5 & 1 & 10 \\
\hline & & 6 & 1 & 152 \\
\hline & \multirow{5}{*}{$90 \%$} & 2 & 1 & 10 \\
\hline & & 3 & 1 & 10 \\
\hline & & 4 & 0.5 & 10 \\
\hline & & 5 & 0.5 & 10 \\
\hline & & 6 & 0.5 & 10 \\
\hline
\end{tabular}




\begin{tabular}{|c|c|c|c|c|}
\hline \multicolumn{3}{|l|}{$\begin{array}{l}\text { LFCM, } \\
s=0.8, N= \\
2\end{array}$} & \multicolumn{2}{|c|}{ Optimal Policy } \\
\hline Forgetting Rate & Learning Rate & Initial time to standard time ratio $(\rho)$ & FAP & Batch Size \\
\hline \multirow{25}{*}{$90 \%$} & \multirow{5}{*}{$70 \%$} & 2 & 5 & 10 \\
\hline & & 3 & 0 & 24 \\
\hline & & 4 & 1 & 10 \\
\hline & & 5 & 1 & 10 \\
\hline & & 6 & 1 & 10 \\
\hline & \multirow{5}{*}{$75 \%$} & 2 & 0 & 20 \\
\hline & & 3 & 1 & 10 \\
\hline & & 4 & 1 & 10 \\
\hline & & 5 & 1 & 10 \\
\hline & & 6 & 1 & 10 \\
\hline & \multirow{5}{*}{$80 \%$} & 2 & 5 & 10 \\
\hline & & 3 & 1 & 10 \\
\hline & & 4 & 1 & 10 \\
\hline & & 5 & 1 & 10 \\
\hline & & 6 & 1 & 10 \\
\hline & \multirow{5}{*}{$85 \%$} & 2 & 1 & 10 \\
\hline & & 3 & 1 & 10 \\
\hline & & 4 & 1 & 10 \\
\hline & & 5 & 1 & 10 \\
\hline & & 6 & 1 & 229 \\
\hline & \multirow{5}{*}{$90 \%$} & 2 & 1 & 10 \\
\hline & & 3 & -1 & 10 \\
\hline & & 4 & 0.5 & 10 \\
\hline & & 5 & 0.5 & 10 \\
\hline & & 6 & 0.5 & 10 \\
\hline \multirow{25}{*}{$87.5 \%$} & \multirow{5}{*}{$70 \%$} & 2 & 5 & 10 \\
\hline & & 3 & 0 & 250 \\
\hline & & 4 & 1 & 10 \\
\hline & & 5 & 1 & 10 \\
\hline & & 6 & 1 & 10 \\
\hline & \multirow{5}{*}{$75 \%$} & 2 & 5 & 17 \\
\hline & & 3 & 1 & 10 \\
\hline & & 4 & 1 & 10 \\
\hline & & 5 & 1 & 10 \\
\hline & & 6 & 1 & 10 \\
\hline & \multirow{5}{*}{$80 \%$} & 2 & 5 & 10 \\
\hline & & 3 & 1 & 10 \\
\hline & & 4 & 1 & 10 \\
\hline & & 5 & 1 & 10 \\
\hline & & 6 & 1 & 10 \\
\hline & \multirow{5}{*}{$85 \%$} & 2 & 1 & 10 \\
\hline & & 3 & 1 & 10 \\
\hline & & 4 & 1 & 10 \\
\hline & & 5 & 1 & 10 \\
\hline & & 6 & 1 & 229 \\
\hline & \multirow{5}{*}{$90 \%$} & 2 & 1 & 10 \\
\hline & & 3 & 1 & 231 \\
\hline & & 4 & 0.5 & 10 \\
\hline & & 5 & 0.5 & 10 \\
\hline & & 6 & 0.5 & 10 \\
\hline
\end{tabular}




\begin{tabular}{|c|c|c|c|c|}
\hline \multicolumn{3}{|l|}{$\begin{array}{l}\text { LFCM, } \\
s=0.8, N= \\
2\end{array}$} & \multicolumn{2}{|c|}{ Optimal Policy } \\
\hline Forgetting Rate & Learning Rate & Initial time to standard time ratio $(\rho)$ & FAP & Batch Size \\
\hline \multirow{25}{*}{$\mathbf{8 5 \%}$} & \multirow{5}{*}{$70 \%$} & 2 & 0 & 250 \\
\hline & & 3 & 5 & 10 \\
\hline & & 4 & 1 & 10 \\
\hline & & 5 & 1 & 10 \\
\hline & & 6 & 1 & 10 \\
\hline & \multirow{5}{*}{$\mathbf{7 5 \%}$} & 2 & 4 & 10 \\
\hline & & 3 & 1 & 10 \\
\hline & & 4 & 1 & 10 \\
\hline & & 5 & 1 & 10 \\
\hline & & 6 & 1 & 10 \\
\hline & \multirow{5}{*}{$80 \%$} & 2 & 5 & 10 \\
\hline & & 3 & 1 & 10 \\
\hline & & 4 & 1 & 10 \\
\hline & & 5 & 1 & 10 \\
\hline & & 6 & 1 & 10 \\
\hline & \multirow{5}{*}{$85 \%$} & 2 & 1 & 10 \\
\hline & & 3 & 1 & 10 \\
\hline & & 4 & 1 & 10 \\
\hline & & 5 & 1 & 10 \\
\hline & & 6 & 1 & 229 \\
\hline & \multirow{5}{*}{$90 \%$} & 2 & 1 & 10 \\
\hline & & 3 & 1 & 180 \\
\hline & & 4 & 0.5 & 10 \\
\hline & & 5 & 0.5 & 10 \\
\hline & & 6 & 0.5 & 10 \\
\hline
\end{tabular}




\subsection{The Hosmer-Lemeshow goodness of fit tests for the binary logistic equations of model $\mathrm{C}$}

- Hosmer-Lemeshow Goodness of fit for the $g(s, \rho, L R, F R)=C^{*}$ binary logistic equation:

Given the following null hypothesis:

$$
H_{0}: \text { The model } g(s, \rho, L R, F R)=C^{*} \text { fits the output data }
$$

the Hosmer-Lemeshow test statistic is calculated as:

$$
\chi^{2}{ }_{H L}=9.47
$$

and the Chi-square distribution at $\alpha=0.05$ and $v=8$ is given by:

$$
\chi_{\alpha, v}^{2}=15.51
$$

$\therefore$ since $\chi^{2}{ }_{H L}<\chi^{2}{ }_{\alpha, \nu}$, there was a failure to reject the null hypothesis that the model $g(s, \rho, L R, F R)=C^{*}$ fits the data at the 0.05 level of significance.

- Hosmer-Lemeshow Goodness of fit for the $g(s, \rho, L R, F R)=F A P^{*}$ binary logistic equation:

Given the following null hypothesis:

$$
H_{0}: \text { The model } g(s, \rho, L R, F R)=F A P^{*} \text { fits the output data }
$$

the Hosmer-Lemeshow test statistic is calculated as:

$$
\chi^{2}{ }_{H L}=6.01
$$

and the Chi-square distribution at $\alpha=0.05$ and $v=8$ is given by:

$$
\chi^{2}{ }_{\alpha, v}=15.51
$$

$\therefore$ since $\chi^{2}{ }_{H L}<\chi^{2}{ }_{\alpha, v}$, there was a failure to reject the null hypothesis that the model $g(s, \rho, L R, F R)=F A P^{*}$ fits the data at the 0.05 level of significance. 